LAWRENCE LIVERMORE NATIONAL LABORATORY

The Effect of Initial Conditions

on the Nonlinear Evolution of

Perturbed Interfaces Driven by Strong Blast Waves

A. R. Miles

May 6, 2004 


\section{DISCLAIMER}

This document was prepared as an account of work sponsored by an agency of the United States Government. Neither the United States Government nor the University of California nor any of their employees, makes any warranty, express or implied, or assumes any legal liability or responsibility for the accuracy, completeness, or usefulness of any information, apparatus, product, or process disclosed, or represents that its use would not infringe privately owned rights. Reference herein to any specific commercial product, process, or service by trade name, trademark, manufacturer, or otherwise, does not necessarily constitute or imply its endorsement, recommendation, or favoring by the United States Government or the University of California. The views and opinions of authors expressed herein do not necessarily state or reflect those of the United States Government or the University of California, and shall not be used for advertising or product endorsement purposes.

This work was performed under the auspices of the U.S. Department of Energy by University of California, Lawrence Livermore National Laboratory under Contract W-7405-Eng-48. 
Title of Dissertation:

Dissertation Directed By:

\section{THE EFFECT OF INITIAL CONDITIONS ON THE NONLINEAR EVOLUTION OF PERTURBED INTERFACES DRIVEN BY STRONG BLAST WAVES}

Aaron R. Miles, Doctor of Philosophy, 2004.

\author{
Dr. M. John Edwards \\ Lawrence Livermore National Laboratory, \\ and Professor Roald Sagdeev, \\ Department of Physics, University of \\ Maryland
}

In core-collapse supernovae, strong blast waves drive interfaces susceptible to Rayleigh-Taylor (RT), Richtmyer-Meshkov (RM), and Kelvin-Helmholtz (KH) instabilities. In addition, perturbation growth can result from material expansion in large-scale velocity gradients behind the shock front. Laser-driven experiments are designed to produce a strongly shocked interface whose evolution is a scaled version of the unstable hydrogen-helium interface in core-collapse supernovae such as SN 1987A. The ultimate goal of this research is to develop an understanding of the effect of hydrodynamic instabilities and the resulting transition to turbulence on supernovae observables that remain as yet unexplained.

In this dissertation, we present a computational study of unstable systems driven by high Mach number shock and blast waves. Using multi-physics radiation hydrodynamics codes and theoretical models, we consider the late nonlinear instability evolution of single mode, few mode, and multimode interfaces. We rely primarily on $2 \mathrm{D}$ calculations but present recent $3 \mathrm{D}$ results as well. For planar 
multimode systems, we show that compressibility effects preclude the emergence of a regime of self-similar instability growth independent of the initial conditions (IC's) by allowing for memory of the initial conditions to be retained in the mix-width at all times. The loss of transverse spectral information is demonstrated, however, along with the existence of a quasi-self-similar regime over short time intervals. Aspects of the IC's are shown to have a strong effect on the time to transition to the quasi-selfsimilar regime.

With higher-dimensional blast waves, divergence restores the properties necessary for establishment of the self-similar state, but achieving it requires very high initial characteristic mode number and high Mach number for the incident blast wave. We point to recent stellar calculations that predict IC's we find incompatible with self-similarity, and emphasize the consequent importance of developing a sound understanding of the initial modal structure in the supernova progenitor.

For divergent and planar systems, the time-dependence of the drive is shown to impose an "effective box size" on the systems that limits the inverse cascade to large-scales. Our model explains the weak IC-dependence of this scale observed in some supernova calculations. 


\title{
THE EFFECT OF INITIAL CONDITIONS ON THE NONLINEAR EVOLUTION OF PERTURBED INTERFACES DRIVEN BY STRONG BLAST WAVES
}

\author{
By \\ Aaron R. Miles \\ Dissertation submitted to the Faculty of the Graduate School of the \\ University of Maryland, College Park, in partial fulfillment \\ of the requirements for the degree of \\ Doctor of Philosophy \\ 2004
}

\author{
Advisory Committee: \\ Professor Roald Sagdeev, Chair \\ Dr. M. John Edwards, Co-Chair \\ Professor Thomas Antonsen \\ Professor Eve Ostriker \\ Professor Edward Ott
}


(C) Copyright by

Aaron R. Miles

2004 


\section{Dedication}

To Marintha, who has given support and endured for six years, and to Aleksei,

Nathaniel, and Ekaterina, who have been waiting all their lives for Daddy to finally

"finish his writing and answer all the questions right". 


\section{Acknowledgements}

I am indebted to several advisors, both official and unofficial. John Edwards, my LLNL advisor, contributed countless hours over the course of three years, and actually read and critiqued every page of every chapter. His active direction, honest criticism, and depth of understanding proved indispensable. Chuan Liu and Roald Sagdeev, both of the University of Maryland, were willing to supervise remotely and provide support despite limited opportunities for personal contact.

The Raptor simulations presented in this dissertation were made possible by the very significant contributions of Jeff Greenough. Lasnex simulations were performed by Dave Braun and John Edwards, and the HYDRA calculation included in the Appendix was done by Steve Weber.

Experiments were performed and analyzed by Harry Robey, Brent Blue, Gail Glendinning, and Freddy Hansen, all from LLNL, and by Paul Drake, Caroline Kuranz, and Derek Leibrandt, all from the University of Michigan. Work at the University of Michigan was supported by the U.S. Department of Energy under grants DE-FG03-99DP00284, DE-FG03-00SF22021 and other grants and contracts.

I also benefited greatly from collaboration with University of Arizona astrophysicist Dave Arnett and is student Casey Meakin. Dave and Casey shared results from their $2 \mathrm{D}$ and 3D stellar calculations and helped guide my work in the direction of greater astrophysical relevance.

Finally, I would like to acknowledge the Livermore Computing staff for providing and maintaining the computational resources without which my work could not have been done. I am in particular indebted to Greg Tomaschke, who somehow 
managed to deliver about seven CPU years of run time in the final two weeks before this dissertation was submitted to the committee. 


\section{Table of Contents}

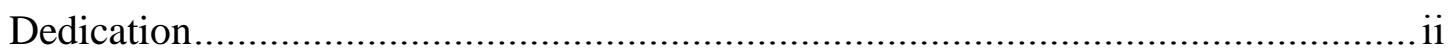

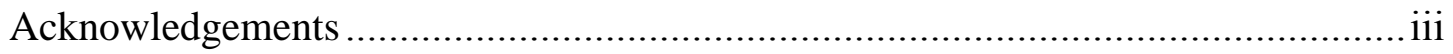

Table of Contents

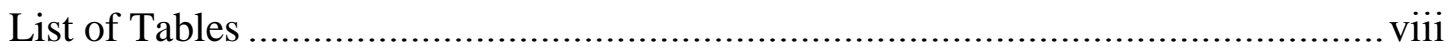

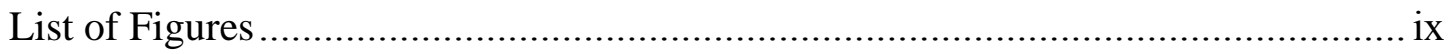

Chapter I: Introduction ............................................................................ 1

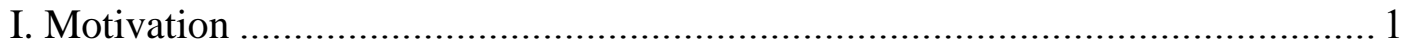

II. Combined approach in HED physics....................................................... 3

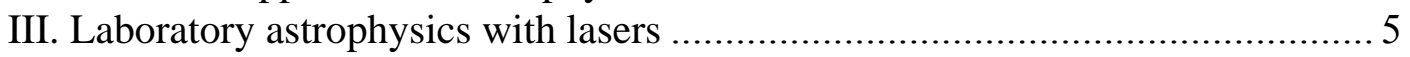

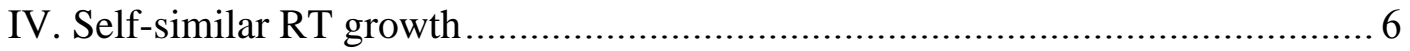

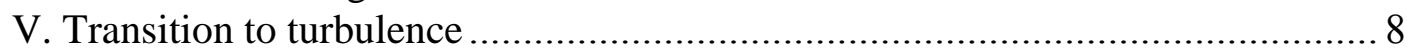

VI. Objectives and methodology ............................................................... 9

VII. Outline of the remainder and summary of conclusions ................................. 10

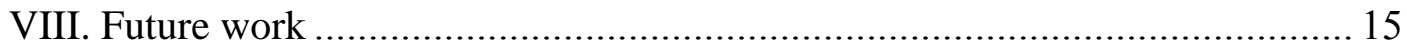

Chapter 2: Numerical simulation of supernova-relevant laser-driven hydro

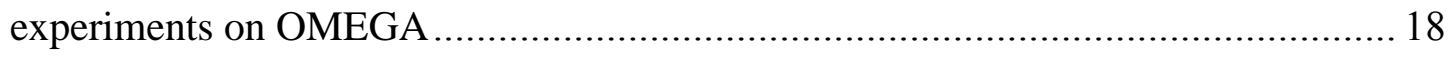

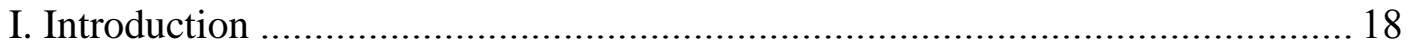

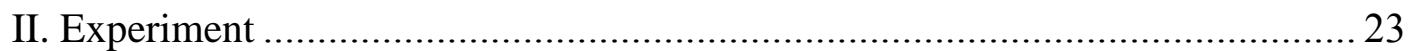

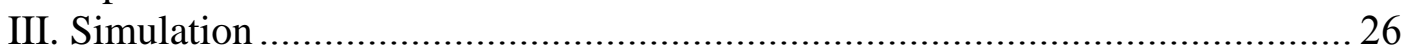

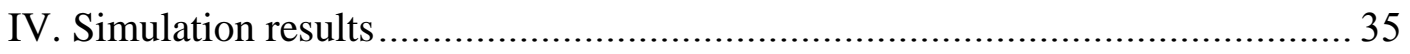

A. Zero-order hydrodynamics .................................................................... 35

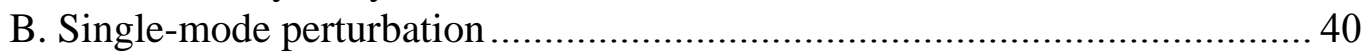

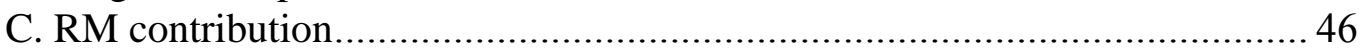

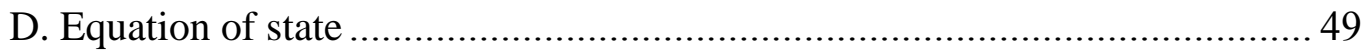

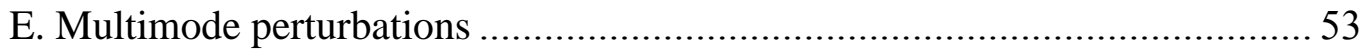

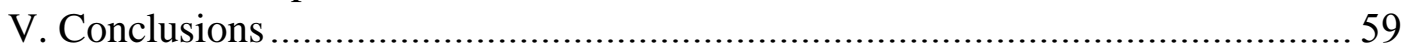

Chapter 3: The effect of a short wavelength mode on the evolution of a long wavelength perturbation driven by a strong blast wave ..............................................6. 62

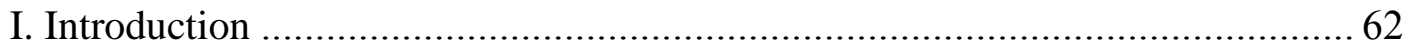

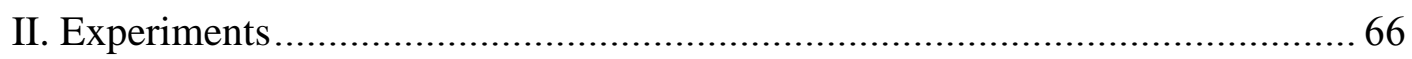

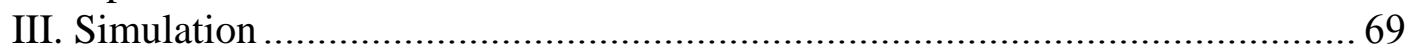

IV. New two-mode interface (short on long) with varying phase ......................... 70

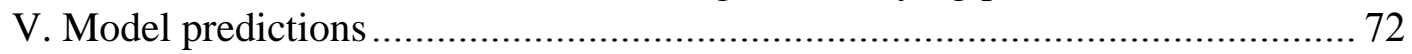

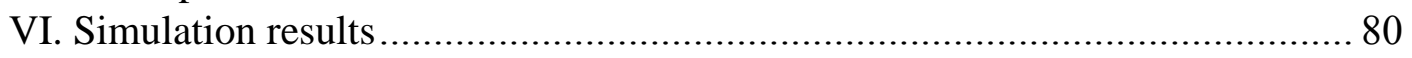

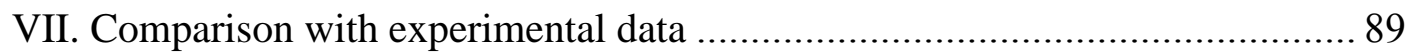

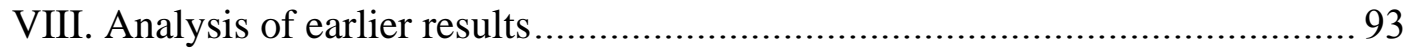

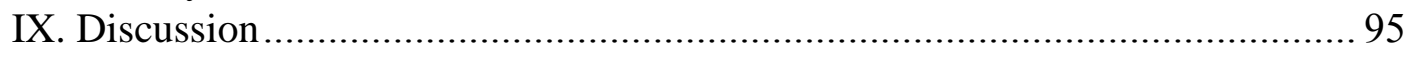

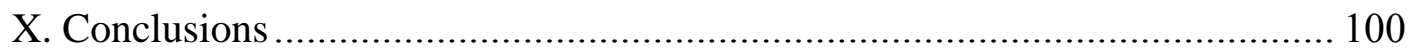

Chapter 4: Bubble merger model for the nonlinear Rayleigh-Taylor instability driven

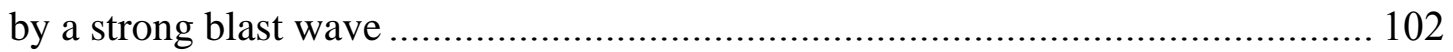

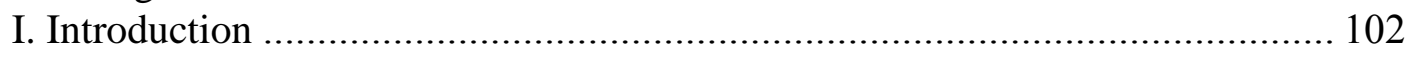




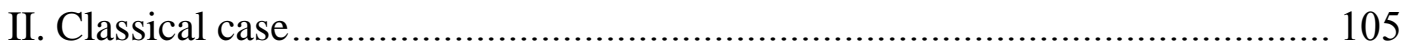

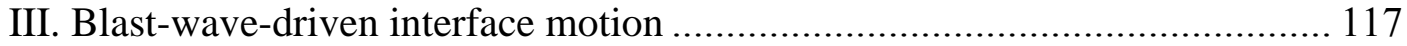

IV. Buoyancy-drag model for blast-wave-driven case....................................... 123

V. Merger model for blast-wave-driven case ……….................................... 132

VI. Statistical-mechanics model for blast-wave-driven case............................... 144

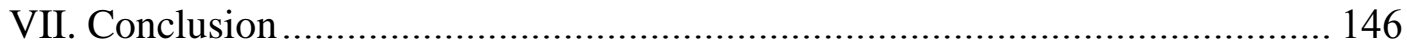

Chapter 5: Effect of Initial Conditions on 2D Rayleigh-Taylor Instability and

Transition to Turbulence in Planar Blast-wave-driven Systems ............................. 148

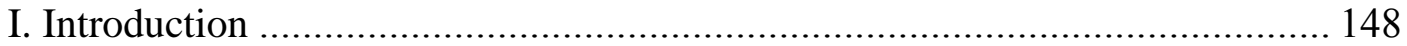

II. Code and calculation setup................................................................... 150

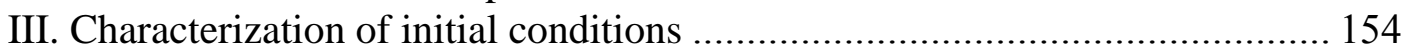

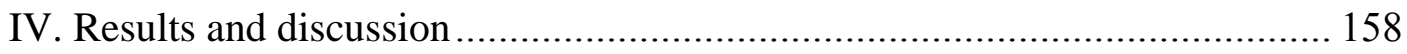

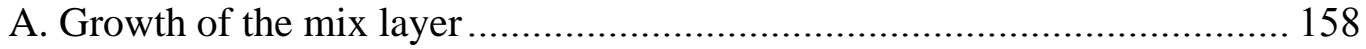

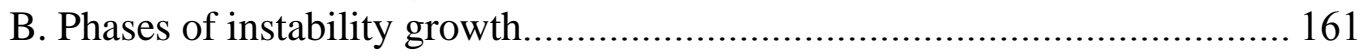

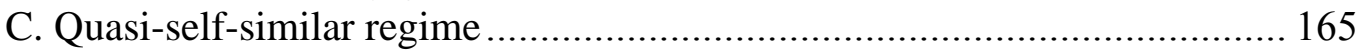

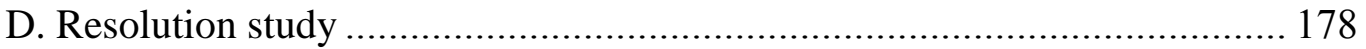

E. Dependence of transition time on initial conditions ………………........... 180

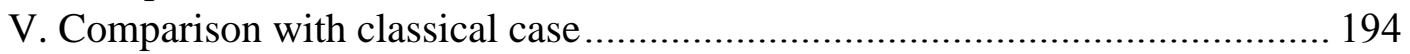

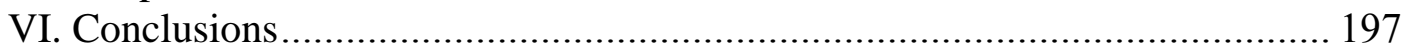

Chapter 6: Transition to Turbulence and Effect of Initial Conditions on 3D

Compressible Mixing in Planar Blast-wave-driven Systems ................................. 200

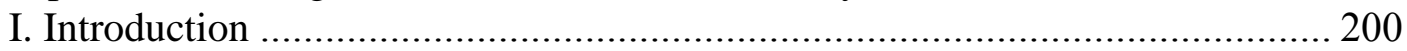

II. Transition to turbulence in RT-instability-driven systems.............................. 202

III. Effect of transition on RT instability growth ............................................ 205

IV. Effect of decompression and drive decay in classical turbulence.................. 208

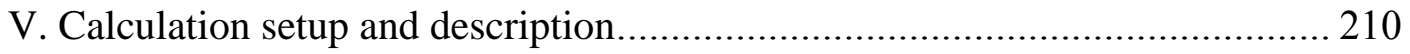

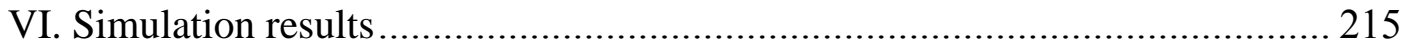

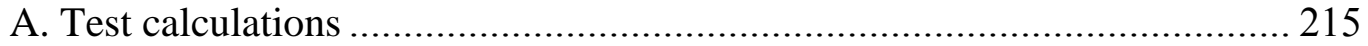

B. Effect of dimensionality on single mode growth...................................... 217

C. Evolution of short-wavelength component …………............................... 220

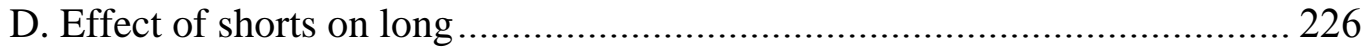

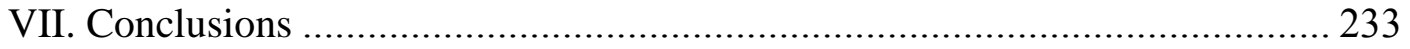

Appendix A. CALE simulation of Richtmyer-Meshkov instability experiments at high

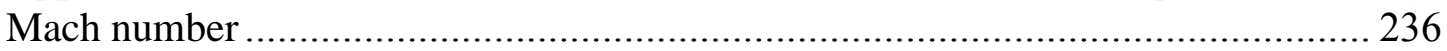

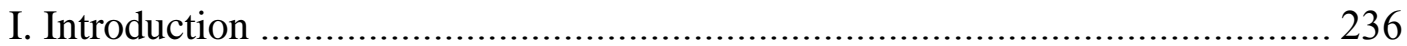

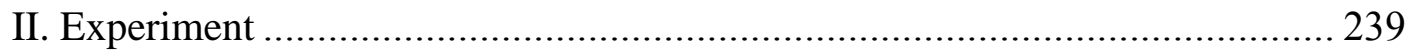

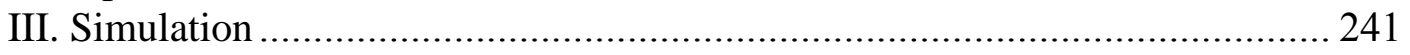

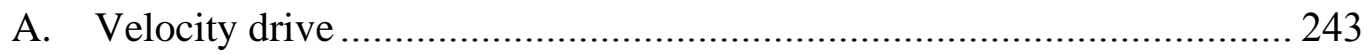

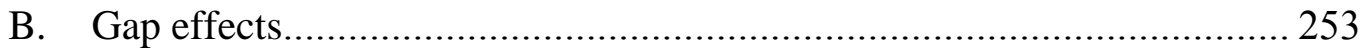

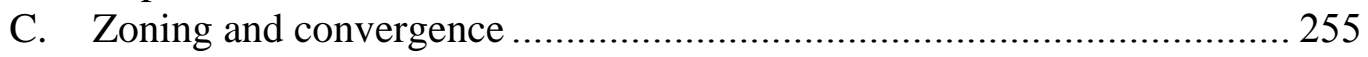

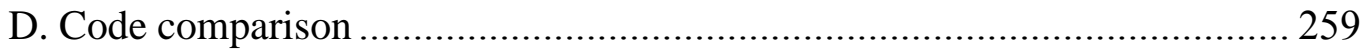

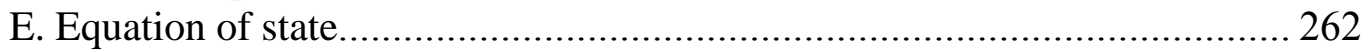

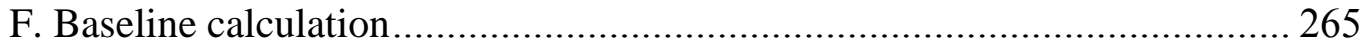

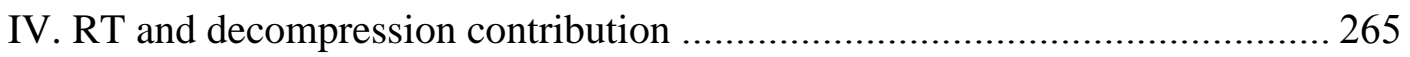

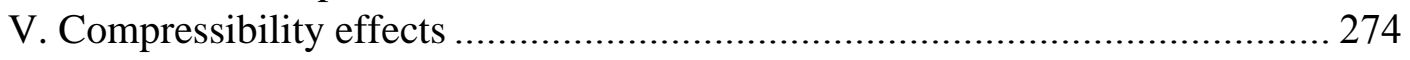




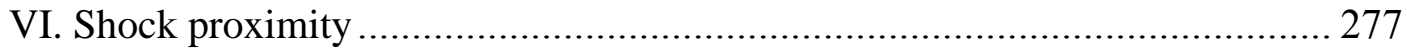

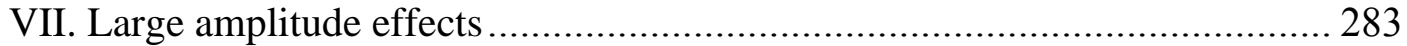

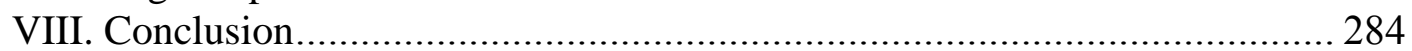

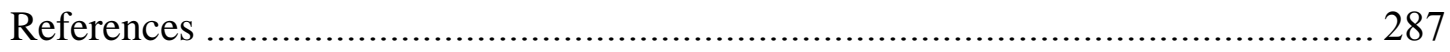




\section{List of Tables}

\section{Chapter 4}

Table 1: List of the parameters used to classify and characterize the initial spectral conditions and a summary of their effect on the nonlinear instability evolution............................................159 


\section{List of Figures}

\section{Chapter 2}

Figure 1: Density and pressure behind a blast wave that has passed through a material interface.....................................................19

Figure 2: Target schematic................................................ 24

Figure 3: Estimation of the effect of preheat................................ 30

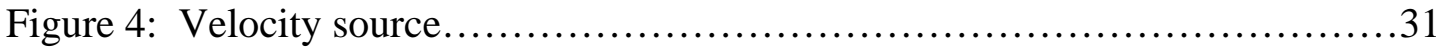

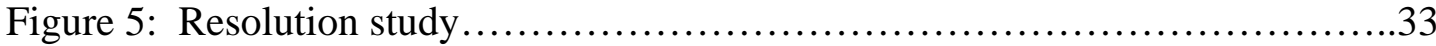

Figure 6: ALE/Eulerian comparison for single and two-mode targets................34

Figure 7: Time-dependence of fluid variables at the plastic-foam interface..........37

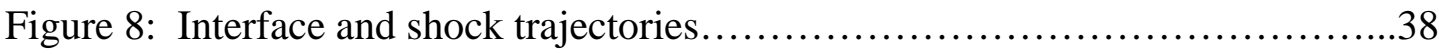

Figure 9: Interface velocity............................................. 40

Figure 10: Single-mode data and simulation.................................. 41

Figure 11: Single mode perturbation.......................................43

Figure 12: Comparison of the simulation results with the buoyancy-drag model....45

Figure 13: Estimation of contribution from RM instability and decompression.....48

Figure 14: Tabular EOS shock Hugoniot curves.............................50

Figure 15: EOS sensitivity.............................................. 51

Figure 16: Equation of state............................................ 52

Figure 17: Two-mode perturbation simulation and data..........................55

Figure 18: Eight-mode perturbation simulation and data.........................57

\section{Chapter 3}

Figure 1: Target schematic..............................................67

Figure 2: Interface velocity and deceleration...................................68

Figure 3: Single and two-mode initial interfaces................................ 71

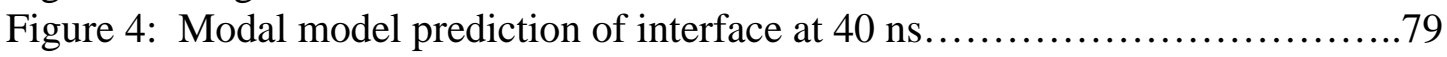

Figure 5: Spike-bubble averaged amplitudes corrected for decompression...........81

Figure 6: Separate spike and bubble amplitudes corrected for decompression.......82

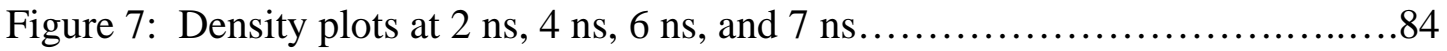

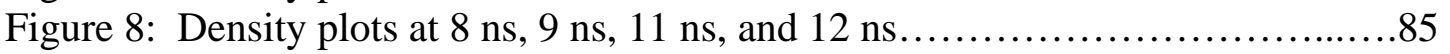

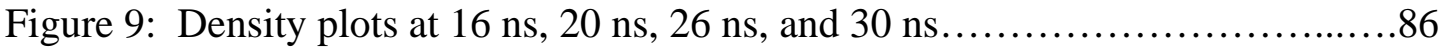

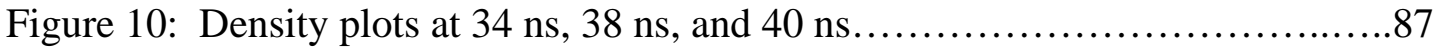

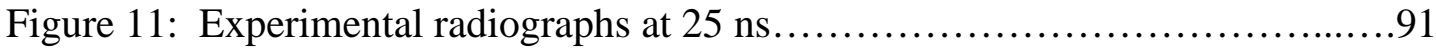

Figure 12: Comparison of data with simulation without decompression effect subtraction..................................................92

Figure 13: Previous two mode simulation and experiment.......................94

Figure 14: Previous eight mode experiment and simulation.....................96 


\section{Chapter 4}

Figure 1: Density and pressure behind a blast wave that has passed through a material interface.....................................................103

Figure 2: Loss of memory of the initial transverse scale........................113

Figure 3: Typical solution to the self-similar spherical blast wave problem..........120

Figure 4: Estimated limiting drive time .........................................126

Figure 5: Application of generalized buoyancy-drag model.......................131

Figure 6: Quasi-self-similar regime........................................138

Figure 7: Minimum mode number in spherical-blast-wave-driven system...........141

Figure 8: Similarities in asymptotic interface structure appear in a variety of systems driven by spherical blast waves.....................................143

Figure 9: Time-dependence of the similarity parameter for the planar case..........145

\section{Chapter 5}

Figure 1: Target schematic and variation in time of interface velocity and deceleration.................................................... 151

Figure 2: Pressure, density, and velocity profiles after refraction of a planar blast wave through a heavy-light interface in the target.......................153

Figure 3: Comparison of pure RM and blast-wave-driven growth rates.............154

Figure 4: Initial spectral shapes and typical interface profiles......................155

Figure 5: Mix width histories and growth velocities from 52 2D simulations.........160

Figure 6: Sensitivity of bubble amplitude and velocity to spectral shape............162

Figure 7: Three phases of instability growth....................................163

Figure 8: "Effective box size" imposed by the decaying nature of the drive.........164

Figure 9: Time-dependence of the similarity parameter...........................166

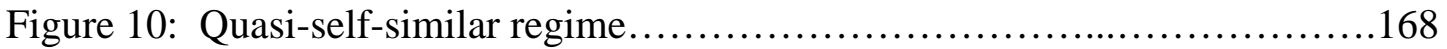

Figure 11: Loss of transverse spectral information...............................170

Figure 12: Transverse and parallel turbulent kinetic energy spectra at $10 \mathrm{~ns} . . . \ldots . . .171$

Figure 13: Transverse and parallel velocity fluctuation spectra at $10 \mathrm{~ns} . \ldots \ldots \ldots \ldots . . .172$

Figure 14: Degree of mixedness for all 52 2D simulations included in Fig. 5......174

Figure 15: Bubble to spike amplitude ratio....................................176

Figure 16: Ratio of transverse to total kinetic energy, integrated over the mix region, from the single mode 4 simulation......................... 177

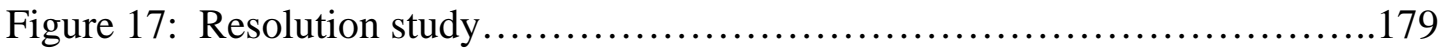

Figure 18: Density, directed turbulent kinetic energy, and directed velocity fluctuation spectra at 0 and $10 \mathrm{~ns}$ for highest resolution (2048 ppb)....181

Figure 19: Dependence of amplitude growth and transition time on the initial rmsamplitude....................................................... 183

Figure 20: Variation of short-wavelength cutoff for flat spectrum..................186

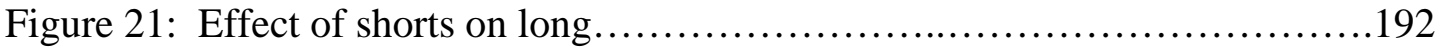

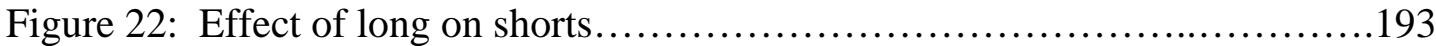




\section{Chapter 6}

Figure 1: Target schematic and variation in time of interface velocity and deceleration..................................................212

Figure 2: Initial spectral shapes used for the short-wavelength component in the 3D calculations ....................................................213

Figure 3: Initial interface contour plots....................................214

Figure 4: Log density slices through a 3D simulation with 2D initial conditions....216

Figure 5: Log density slices through a 3D simulation with initial conditions given by

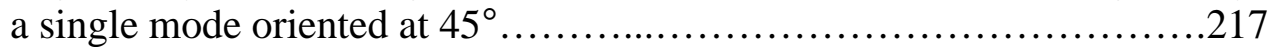

Figure 6: Effect of dimensionality on single-mode growth.....................218

Figure 7: Late-time log density image from mode 4 "egg-carton" interface..........219

Figure 8: Perturbation amplitude and velocity histories from shorts-only cases.....221

Figure 9: Mixing parameter and log density plots from 2D and 3D ng shorts-only calculations....................................................223

Figure 10: Time evolution of density spectra $\rho_{\mathrm{k}}, \mathrm{k}^{5 / 3} \rho_{\mathrm{k}}$, and turbulent directed energy spectra..................................................224

Figure 11: Perturbation amplitude and velocity histories from calculations with mode

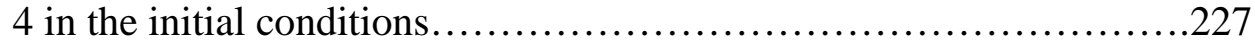

Figure 12: Mixing in simulations with mode 4 in the initial spectra..............228

Figure 13: Log density plots of the evolving mix layer from the 3D m4 plus 3D narrow gaussian shorts calculation.............................229

Figure 14: Density and energy spectra from the 3D m4 plus 3D narrow gaussian shorts calculation..............................................230

Figure 15: Log density slices of the evolving mix layer from the 2D m4 plus 3D narrow gaussian shorts calculation.............................231

Figure 16: Density and energy spectra from the 2D m4 plus 3D narrow gaussian shorts calculation ............................................. 232

\section{Appendix A}

Figure 1: Target schematic...............................................240

Figure 2: Time-dependent velocity source obtained from 1 and 2D LASNEX simulations....................................................243

Figure 3: Comparison of simulations driven by velocity source from 1 or 2D LASNEX calculation..............................................244

Figure 4: Effect of velocity source details on amplitude history..................246

Figure 5: Separate spike and bubble amplitudes and growth rates..................247

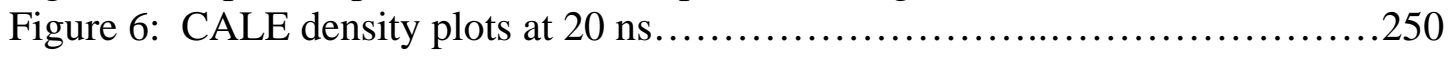

Figure 7: Comparison of experimental radiograph at $20 \mathrm{~ns}$ with CALE simulated radiographs...................................................250

Figure 8: Results with new source from 2D LASNEX simulation with improved laser pulse shape............................................252

Figure 9: Perturbation amplitude and growth rate with and without air gap..........253 
Figure 10: Density plots (at $20 \mathrm{~ns}$ ), radiographs, and spike and bubble velocities for simulations with and without an air gap........................254

Figure 11: Section of computational domain showing rectangular and conforming computational mesh..........................................256

Figure 12: Amplitude resolution study with rectangular and conforming mesh.....257

Figure 13: Eulerian / ALE comparison......................................258

Figure 14: Code comparison of simulation of Aleshin shock-tube experiment......260

Figure 15: Test problem: Comparison of CALE and RAPTOR simulation amplitude histories with and without an air gap..............................261

Figure 16: Comparison of CALE and RAPTOR simulation density plots at $20 \mu \mathrm{s}$ with and without an air gap.....................................261

Figure 17: Effect of equation of state, drive, and gap on transmitted shock proximity

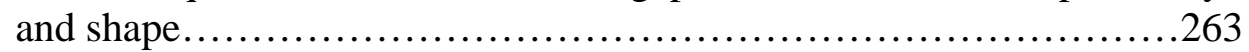

Figure 18: Distance from bubble and spike to transmitted shock vs. perturbation amplitude.....................................................264

Figure 19: RT / decompression effects...................................268

Figure 20: RT / decompression effects for small (7 $\mu \mathrm{m})$ initial amplitude driven by constant velocity $(14 \mu \mathrm{m} / \mathrm{ns})$ drive...........................269

Figure 21: RT / decompression effects for large $(22 \mu \mathrm{m})$ initial amplitude driven by

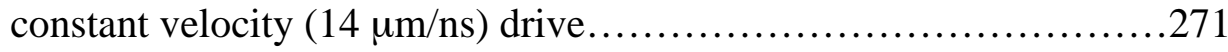

Figure 22: RT / decompression effects for large initial amplitude $(22 \mu \mathrm{m})$ driven by 6 ns constant velocity $(14 \mu \mathrm{m} / \mathrm{ns})$ drive...........................273

Figure 23: RT contribution in simulation with real drive (L2D) .................274

Figure 24: Model predictions and simulations for large and small initial amplitude with pure RM drive..........................................276

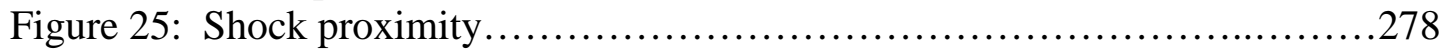

Figure 26: Image vortex model (IVM) prediction.......................... 280 


\section{Chapter I: Introduction}

\section{Motivation}

The appearance of Supernova 1987A in the Large Magellanic Cloud marked the beginning of a change in the way people think about the violent endpoint of massive stars. Although it had been known for some time that the layered structure of the progenitor should be hydrodynamically unstable during the explosion, ${ }^{1,2}$ the assumption of spherical symmetry was almost always incorporated into models and otherwise reflected in the paradigm of core-collapse supernovae. This was due in large part to the practical limitations of multidimensional numerical calculations. But when heavy elements originating from the core of SN 1987A appeared at the photosphere six months earlier than predicted by one-dimensional explosion models, it became clear that something significant was being neglected. ${ }^{3}$ Since then, evidence that asymmetry is the rule in core-collapse supernovae has continued to accumulate, ${ }^{4,5}$ and multidimensional computer codes have been developed and applied to the problem in an effort to understand proposed asymmetry mechanisms. Several ideas have been put forward, and two prominent theories have emerged.

First of all, it has been suggested that the action of a magnetorotational mechanism during collapse can lead to the formation of energetic jets in the star's 
central regions. ${ }^{6}$ As they race outward, these jets drive shocks that destroy the star and transport core material much faster than in a spherical explosion.

The second explanation is based on the hydrodynamic instability of perturbed interfaces when subjected to a reversal of pressure and density gradients. Due to processes such as convective stirring and localized thermonuclear burn, boundaries between layers of different materials within the star are unlikely to be perfectly smooth. Even if the shock wave produced as the core rebounds against neutron degeneracy pressure is initially spherical, it can drive the amplification of any such preexisting perturbations. After passage of the shock front, this interface evolves into a complicated structure of outward-growing spikes of heavier material and infalling "bubbles" of lighter elements. ${ }^{7-10}$ Late in time, these spikes can move far ahead of what a 1D model would predict as the interface position and might explain the anomalously early appearance heavy elements at the photosphere.

Hydrodynamic instabilities have been observed in a variety of astrophysical systems in addition to core-collapse supernovae. On the earth as well, they are familiar phenomena. The Rayleigh-Taylor (RT) instability, ${ }^{11,12}$ which results when a heavier fluid is supported by a lighter fluid against gravity, explains the exit of water from an overturned glass. Velocity shear drives perturbation growth and rollup via the Kelvin-Helmholtz (KH) instability, which can be observed in clouds. The RichtmyerMeshkov (RM) instability ${ }^{13,14}$ results when a shock wave crosses a perturbed interface, whether from light to heavy or from heavy to light. The RM instability is not so apparent in everyday experience, but can be thought of as the impulsive limit of the RT instability. 
When a blast wave crosses an interface from a heavier to a lighter material, all three of these instabilities are typically present. Since this is the case in core-collapse supernovae, all three are likely active contributors to accelerated mixing and transport of material. They come together in the laboratory as well when high-powered lasers are used to drive the implosion of sub-millimeter capsules of thermonuclear fuel. In such inertial confinement fusion (ICF) applications, laser energy is converted into shocks that travel inward from the capsule's surface. ${ }^{15}$ The resulting implosion is something like a supernova in reverse. The ultimate goal of this program is to use these converging shocks to establish conditions at the center capable of temporarily sustaining thermonuclear reactions, thereby potentially tapping an inexhaustable source of energy. Material interfaces within the capsule are hydrodynamically unstable, however, and the instability growth can completely quench the reactions. In double shell ignition capsules currently under development for the National Ignition Facility (NIF) laser, this growth might lead to the formation of a turbulent mixing zone (TMZ) similar to that expected to occur in supernovae. ${ }^{16}$

\section{Combined approach in HED physics}

The development of an instability-driven turbulent mixing layer, including the transition from a more ordered early-time state, is a very complicated problem that is at best only partially understood. But when it occurs in astrophysical and ICF applications, it is generally only one part of an immensely complicated system. The dynamics of these systems is governed by the nonlinear equations of compressible 
hydrodynamics (the Euler or Navier-Stokes equations) in regimes far removed from linear approximations. Turbulence provides a mechanism for indirect interactions of the largest scales of interest with the very small dissipative scales. In addition to instabilities and turbulence, there are shocks of both high and low Mach numbers, as well as multiple fluid species and contact discontinuities. In many cases, interaction of radiation with the fluid is important and must also be accounted for. These coupled radiation hydrodynamics equations must be solved for matter under extreme pressure and temperature conditions. In this high energy density (HED) regime where thermal pressures are at least about a million atmospheres, equations of state (EOS) and opacities are often not well approximated by simple models. Finally, the complex physics of thermonuclear reactions and burn, laser-plasma interactions, and non-local thermodynamic equilibrium potentially must also be included.

The complexity of these multi-physics systems has motivated a combined approach involving the application of computer simulations, laboratory experiments, simplified models, and astronomical observations. Models of varying complexity are used to make direct connections between ideas, observations, and experiments on the one hand and the relevant equations on the other. Simulations are used as platforms on which questions of multi-physics interactions can be addressed and theoretical predictions can be tested against experiments and observations. Moreover, they provide a level of data not accessible in either experiments or observations. Finally, HED laser-driven laboratory experiments provide a means of validating astrophysical codes and theories under conditions that are otherwise difficult or impossible to reproduce on earth. The material properties that must be input into the codes, 
including EOS data and opacities, can often be obtained only through such experiments.

\section{Laboratory astrophysics with lasers}

A deep connection can be made between laboratory experiments and astrophysical systems such as supernovae because the governing equations are scale invariant under certain conditions. Despite huge differences in length and time scales, scaled versions of some astrophysical systems can be fielded on high energy density facilities ${ }^{17,18}$ such as the OMEGA laser at the University of Rochester's Laboratory for Laser Energetics (LLE) ${ }^{19}$ and the NIF laser at Lawrence Livermore National Laboratory.

In ongoing experiments aimed at studying instability-driven mixing under supernova-relevant conditions, laser energy is used to drive high Mach number planar shock and blast waves into one end of millimeter-scale cylindrical targets. ${ }^{20-28} \mathrm{~A}$ typical target consists of a more dense plastic section and a less dense foam section, with a prescribed perturbation machined into the plastic at the plastic/foam interface. After the passage of the shock, the interface is unstable and evolves under the combined influence of RT, RM, and KH instabilities. Additional laser beams directed on high-Z backlighter foils yield $\mathrm{x}$-rays that pass through the target and are used to image the developing instability.

There are several advantages of these laser-driven hydro instability experiments relative to other platforms such as shock tubes. First of all, they involve 
Mach numbers that are an order of magnitude higher than those currently possible with shock tubes. This allows them to access more extreme pressure, density, and temperature states. In addition, because the target materials typically begin as solids rather than gases, laser-driven experiments allow for more control over and better characterization of the initial perturbations without complications from membranes or retracting plates. Control over the initial conditions is crucial in studies such as ours that are aimed at linking the late-time nonlinear interface structure back to the initial perturbation spectrum.

\section{IV.Self-similar RT growth}

For classical RT systems comprised of incompressible fluids under constant acceleration, it is widely believed that memory of the initial conditions is lost at late times after the establishment of a self-similar regime. ${ }^{29-31}$ This idea ultimately is based on the simple fact that larger bubbles rise faster than smaller bubbles, and can be

explained in terms of bubble competition and merger. ${ }^{32,33}$ As a larger bubble rises above its smaller neighbor, it is free to expand laterally, eventually filling the space previously occupied by its neighbor. Material flowing around the larger bubble and into the spikes below sweeps the smaller bubble downstream. This process leads to the continual generation of larger, faster moving objects and an acceleration of the bubble and spike fronts. Eventually, the interface is dominated by structures resulting from many successive generations of bubble merger rather than from the unstable growth of preexisting perturbations. Loss of memory of the initial conditions means 
that the statistical properties observed in the late-time interface could have arisen from a wide range of initial perturbation spectra. If the initial conditions are forgotten, the height of the bubble front as well as the dominant transverse scale must grow in proportion to $\mathrm{gt}^{2}$, where $\mathrm{g}$ is the acceleration and $\mathrm{t}$ is time, as this is the only length scale remaining in the problem. The interface can be described in a statistical sense by a bubble-size distribution function. In the self-similar or scale-invariant regime, this function does not change in time except for a scale factor proportional to the characteristic bubble size. Thus any initial distribution must evolve in time towards the scale-invariant distribution.

Although the idea of self-similar RT growth is well motivated, it has yet to be demonstrated conclusively. If the self-similar regime does exist, then it is certainly difficult to reach in simulations and diagnosable experiments. The $\mathrm{gt}^{2}$ scaling is indeed observed, but there is a great deal of disagreement and debate about the constant of proportionality $\alpha \cdot{ }^{34}$ In addition, simulations tend to give a value for $\alpha$ that is only about half as large as values extracted from experiments. This disagreement is significant because $\alpha$ could in principle be a function of the initial conditions, indicating that they are never completely forgotten. Other proposed explanations for the observed variations in $\alpha$ typically reduce to either limitations in time (not enough time for complete memory loss) or space (self-similar generation of larger scales inhibited by walls or by spurious long wavelength modes present in the initial conditions).

Despite limited understanding of nonlinear instability evolution in classical RT systems, the ideas of self-similar growth are sometimes invoked in discussions of 
blast-wave-driven instabilities in core-collapse supernovae. Even if valid in the classical case, one should question to what extent these ideas would apply in the more complicated blast-wave-driven case, where RM is present, the acceleration is timedependent, and the flow is compressible.

\section{Transition to turbulence}

Whether or not there is a true self-similar regime, hydrodynamically unstable systems certainly can undergo a transition from an early time, more ordered structure to a late time structure that is disordered and appears random. If the Reynolds number is sufficiently high and sufficient time is allotted, this late-time structure will be turbulent.

Many unanswered questions remain, however, about the requirements for transition to the turbulent or turbulent-like state and its relationship to loss of memory of initial conditions. In particular, the effect of the initial conditions on the transition is not well understood. In addition, it is also important to understand what effect the transition has on the instability growth rate. For 3D systems, it has been noted that there will be a competition between the continual generation of larger, faster growing structures and the tendency of increased turbulent dissipation to inhibit the growth. ${ }^{35}$ To date, 2D simulations of mixing in Type II supernovae produce spike velocities about a factor of two smaller than the observed velocities of heavy core elements beyond the photosphere. ${ }^{36}$ It remains unclear whether or not this discrepancy can be 
resolved once $3 \mathrm{D}$ effects, including the transition to turbulence, are included in highresolution calculations.

\section{Objectives and methodology}

In this dissertation, we examine the effect of initial spectral conditions on the nonlinear instability evolution of an interface driven by a strong blast wave. This broad objective encompasses four principle goals, each of which will be developed in subsequent chapters:

(1) Develop a validated computational platform with which to study nonlinear blast-wave-driven instability evolution in planar laser-driven systems.

(2) Understand (using simulations, simple models, and comparison with experiments and observations) similarities and differences between the blastwave-driven instability and classical RT.

(3) Understand how the details of the initial spectrum influence the nonlinear growth of 2D and 3D perturbations, including the transition to turbulence.

(4) Evaluate the relevance of current experiments with respect to supernova hydrodynamics and suggest directions for future HED experiments that capture the supernova-relevant phenomena and go beyond the limitations of current state of the art simulations and simple models. 
At the heart of this work are multidimensional, high-resolution numerical simulations performed with two proven radiation hydrodynamics codes called $\mathrm{CALE}^{37}$ and Raptor. ${ }^{38}$ Both were developed at Lawrence Livermore National Laboratory, with Raptor based on earlier work by Bell et al. ${ }^{39}$ Both solve the Euler equations for multi-fluid systems and have extensive multi-physics capabilities. CALE is a 2D arbitrary lagrangian-eulerian finite-differencing code, while Raptor uses a higher-order Godunov method and adaptive mesh refinement (AMR) in 2 or 3D. Besides its 3D capability, Raptor benefits from parallelization and was run on as many as 512 processors simultaneously.

Despite the focus on simulations, we draw extensively on all aspects of the combined approach described above. We benefit particularly from close ties with ongoing experiments, and have been able to contribute to the design and analysis of experiments conducted at the Omega laser facility and planned for the NIF.

\section{Outline of the remainder and summary of conclusions}

This introduction is followed by five additional chapters and one appendix. The evolution from one chapter to the next follows a natural progression: from 2D CALE simulations of single and few-mode Omega experiments, to a model of multimode interface evolution for blast-wave-driven systems, and finally to 2 and 3D Raptor simulations of multimode systems under NIF-like drive conditions. Despite this ordering, each of these sections is completely self-contained, so that they can in principle be read in any order. In particular, those not interested in the necessary but 
tedious task of detailed code validation against experiment may omit Chapter II and Appendix A. Chapter III, which is a description of our buoyancy-drag model for blast-wave-driven systems, can certainly be read in isolation. The final two chapters on multimode simulations also comprise a self-contained story. Together with Chapter III, they include our most important scientific results. In our opinion, the only downside of this approach is that it leads to some overlap among the chapters, particularly in the introductory sections and general descriptions of codes and experiments. Because of the flexibility it offers, we consider this a worthwhile tradeoff.

With this qualification, we turn to a more detailed outline of the remaining chapters and a brief summary of our results:

In Chapter II and Appendix A, we describe in detail comparisons of CALE simulations with Omega RM (Appendix A) and blast-driven RT (Chapter II) experiments. Through consideration of a host of physical processes and parameters including drive details, $\mathrm{x}$-ray preheat, and equation of state, we identify what should be included in the calculations in order to match the observations. Together with zoning and convergence studies and code-to-code comparisons, these considerations represent the establishment of a validated computational platform that can be used as a test-bed for related calculations where experimental data is not yet available.

Chapter III describes a series of two-mode CALE simulations intended to study the effect of a single short-wavelength secondary mode on the nonlinear evolution of a long-wavelength primary mode. The simulations predict that the shortwavelength "noise" has a dramatic impact on the late-time large-scale interface 
structure, and that this effect is very different depending on whether the secondary mode is in phase or out of phase with the primary mode. For simple two-mode or few-mode systems, we show that details of the initial conditions can be remembered well into the deep nonlinear phase. We explain this sensitivity to initial conditions in terms of diversion and interactions of developing spikes, which leads to the formation of fast jets when commensurate modes are in phase and to the breakup of the primary spikes when they are out of phase.

These predictions led to the design of short-on-long experiments that were subsequently carried out at the Omega laser. The experiments confirm that the presence of the short-wavelength mode can lead to a much more disordered state that is easily distinguished from the single-mode case.

In Chapter IV, we develop a model that describes the evolution of a blastwave-driven multimode interface in terms of bubble competition and merger. Our model goes beyond previous work by including the effects of material decompression and stretching behind the shock front for both planar and divergent systems. On the basis of this model, we are able to show that self-similarity and loss of initial conditions might be possible in divergent systems such as supernovae but not realizable in planar systems such as most laser-driven experiments intended to study mixing in supernovae. The difference arises because modes in divergent systems undergo transverse in addition to radial stretching. Because the time dependence of the stretching is the same in both directions, the ratio of transverse to parallel scales is preserved.

For planar systems, we predict a quasi-self-similar regime during which the 
instability evolution is approximately self-similar over a limited period of time. During this regime, the ratio of characteristic wavelength to perturbation amplitude decreases slowly in time rather than approaching a constant asymptotic value.

Even in the divergent case, loss of initial conditions is possible only for systems with very small-scale initial conditions driven by very high Mach number blast waves. Based on recent stellar calculations, ${ }^{36.40}$ we predict that initial mode numbers present in supernova progenitors are probably not high enough to reach the self-similar regime. If these predictions are correct, the late-time interface structure observed in supernova remnants likely depends strongly on the initial conditions present within the star at the time of explosion.

Finally, we show that the finite duration of the blast-wave drive sets a maximum scale that can be generated on a given interface. For divergent systems, this corresponds to a minimum mode number that depends weakly on the incident Mach number and initial mode number as long as both are sufficiently high.

In Chapters V and VI, we turn our attention from few-mode systems to broadbanded multimode initial conditions, and from the single processor 2D CALE to the 2 and 3D parallelized AMR code Raptor. The effect of initial conditions on nonlinear evolution and transition to turbulence in planar blast-wave-driven systems is considered for 2D perturbations in Chapter V and 3D perturbations in Chapter VI.

In Chapter V, we report on a series of over 70 high-resolution 2D Raptor simulations of planar blast-wave-driven systems under NIF-like drive conditions. In agreement with our model, the perturbation growth shows no apparent approach to a self-similar regime independent of the initial conditions. We also demonstrate the 
effective box size due to drive decay, which sets a maximum transverse scale that can be generated, and the quasi-self-similar regime. This regime is found to exist after the generation of scales larger than the initial conditions but before the effective box size is reached.

The existence of the quasi-self-similar state and the drive-imposed effective box size make the blast-wave-driven case distinct from classical RT. However, we show that transition to the quasi-self-similar state is very similar to its classical counterpart. In both cases, it is marked by an increase in the degree of small-scale mixing, a decrease in the spike velocity, and often an increase in the bubble velocity. We find some indication that the transition takes place slightly sooner in the blastwave-driven case, possibly due to shock-deposited vorticity.

We consider both single component and bi-component (short on long) spectra, and identify several parameters that classify and characterize the initial conditions. A subset of these parameters is studied in order to see how they can affect the observable properties of the deep nonlinear instability evolution. We find, for example, that a long wavelength mode can inhibit the development of small scales and delay the transition to a turbulent-like state when its amplitude is sufficiently large.

Significantly, we show that apparently random variations observed in latetime amplitudes and growth rates are not well correlated with initial spectral shape. Only the average spectral properties are important, such as the initial rms amplitude and characteristic wavenumber. This bodes well for simulations of similarly stronglydriven systems that leave a portion of the short-wavelength end of the spectrum 
unresolved. As long as the system contains some fast-growing and interacting modes that can be resolved computationally or reproduced experimentally (and has the correct initial rms amplitude), the late-time instability evolution will likely closely resemble the fully resolved or complete system. This reaffirms the hope that laserdriven experiments can serve as useful and relevant platforms for studying compressible mixing in supernovae despite their drastically more limited available range of scales. Similarly, carefully-designed numerical simulations need not necessarily reproduce the full range of spectral details present in their physical counterparts in order to reasonably reproduce the late-time large-scale interface structure. These conclusions apply in particular to systems with long-wavelength modes large enough in amplitude to reach the nonlinear phase early on.

In Chapter VI, we present results from 3D calculations of systems that are otherwise identical to those discussed in Chapter V. In agreement with 3D classical RT calculations performed by others, we find more fine-scale mixing in 3D and note that the post-transition bubble growth is reduced relative to that observed in the $2 \mathrm{D}$ calculations. The spike growth, however, does not appear to be inhibited and might even be enhanced. This is particularly significant in light of the fact that $2 \mathrm{D}$ supernova calculations that invoke instability-driven mixing to explain enhanced transport of heavy core elements consistently underpredict the late-time spike velocities by about a factor of two.

\section{Future work}


We hope to continue this research along several parallel directions directly applicable to both supernovae and ICF applications. One obvious aspect of this program is to continue the 3D calculations and analysis described in Chapter VI with the goal of establishing the process that leads from spike interaction and breakdown to the subsequent $3 \mathrm{D}$ turbulent mixing transition. In addition, we would like to further investigate the effect of spike interactions on their velocity distribution in order to determine the extent to which spike material can be accelerated towards the shock front. These simulations are important for the planned extension of the existing series of single and few mode Omega targets to broadbanded NIF experiments. With the greater energy, temporal, and spatial scales afforded by the NIF laser, these experiments will potentially be capable of unambiguously demonstrating transition to 3D turbulence, the generation of larger scales through multiple generations of bubble merger, and the late-time freeze-out stage. Through collaboration with astrophysicists studying supernova progenitors, we will attempt to incorporate realistic initial spectra into the experiments.

A parallel effort would involve the design of planar experiments aimed at studying turbulent mixing in double-shell ignition targets. One essential aspect of these experiments would be the presence of multiple unstable interfaces capable of interacting with one another. Similar interactions are likely important in supernovae, but have typically not been incorporated into the experiments. We expect that the planar double-shell targets can be designed so as to be directly relevant to both systems. 
Planar experiments are particularly valuable because of their improved diagnosability relative to spherical systems and their ability to better maintain high energy density in the absence of divergence. However, our buoyancy-drag model has suggested that the absence of divergence changes the nature of the instability evolution by ensuring that memory of the initial conditions is retained in the perturbation amplitudes at all times. Consequently, we are interested in developing a divergent platform for supernova-relevant compressible mixing experiments. Recent studies at Livermore involving laser-driven spherical blast waves in gases ${ }^{41}$ suggest the lab's Janus laser as a promising near-term candidate.

Finally, we note that current ICF diagnostics in general and x-ray radiography in particular are not optimized for detailed studies of 3D turbulence in laser-drive targets. The long-term success of this program will ultimately depend on the development of innovative new experimental techniques. 


\section{Chapter 2: Numerical simulation of supernova-relevant laser-driven hydro experiments on OMEGA}

\section{Introduction}

The basic Rayleigh-Taylor (RT) instability criterion, ${ }^{1,2}$ neglecting certain potentially stabilizing factors such as surface tension ${ }^{3}$, is the existence of anti-parallel components of pressure and density gradients $(\nabla \mathrm{P} \bullet \nabla \rho<0)$. When this condition is met at an interface between two materials, perturbations on the interface will grow in time. In the inviscid limit, the instability develops exponentially while the perturbations remain small (during the linear phase) with a growth rate given by ${ }^{4}$

$$
\gamma=\sqrt{\frac{k g A}{1+k L}},
$$

where $\mathrm{k}$ is the perturbation wavenumber, $\mathrm{g}$ is the acceleration, $\mathrm{A}$ is the Atwood number, and $\mathrm{L}$ is the density gradient scale length at the interface. At later times, initially sinusoidal perturbations grow into spikes of heavier fluid "falling" into lighter fluid and bubbles of lighter fluid "rising" into heavier fluid. For $A=1$, the bubbles rise with constant (terminal) velocity while spikes fall with constant acceleration in the nonlinear regime. ${ }^{5,6}$ When $\mathrm{A}<1$, the spike eventually also reaches terminal velocity. ${ }^{6}$

There are several important non-gravitational systems that are also strongly affected by the RT instability. For example, the RT instability has played an 


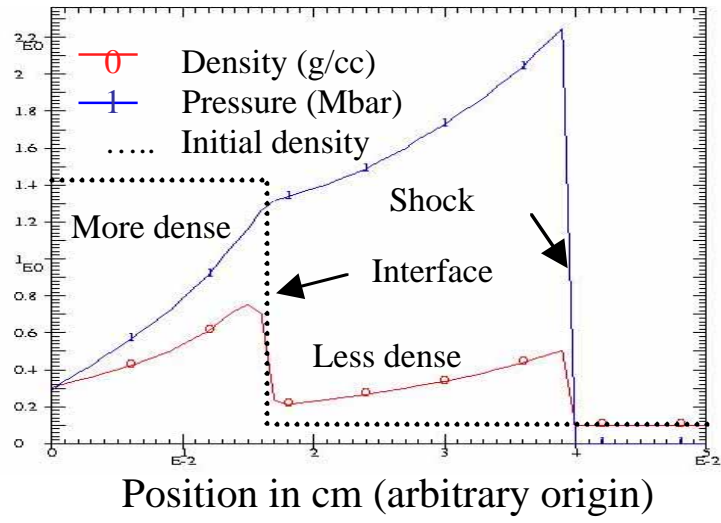

Figure 1: Density (curve 0) and pressure (curve 1) behind a blast wave that has passed through a material interface from a $1.42 \mathrm{~g} / \mathrm{cc}$ plastic to a $0.1 \mathrm{~g} / \mathrm{cc}$ foam. The interface is RT unstable due to the presence of antiparallel density and pressure gradients at the interface. The dotted line shows the initial (pre-shock) density profile. The data are from a 1D CALE simulation of the experiment-relevant planar hydrodynamics at 14 ns.

important historical role in the ability to magnetically confine hot plasmas, ${ }^{7}$ where the plasma serves as the heavier fluid. The confining magnetic field plays the role of the lighter fluid supporting the plasma against pseudo-gravitational centrifugal forces.

The RT instability criterion can also be satisfied at a material interface through which a blast wave has been transmitted from a heavier to a lighter fluid. ${ }^{8}$ As is illustrated in Fig. 1, since the pressure behind a blast wave is always falling in time at any fixed point and in distance behind the shock front (at least in the self-similar regime $^{9}$ ), an interface generally becomes RT unstable when it transmits a blast wave down a density gradient (ie from more dense to less dense material).

In inertial confinement fusion (ICF), pellets containing thermonuclear fuel are imploded by laser or particle beams or by x-rays generated by the interaction of such beams with the high- $Z$ walls of a hohlraum. ${ }^{4}$ The driving shock waves pass through the various material interfaces within the target, resulting in interface accelerations 
and decelerations that can in turn drive the RT instability. These processes tend to break up material shells and mix cold outer layers with the hot central region containing the fuel, and the resulting reduction in thermonuclear yield can be significant or even complete. Consequently, the RT instability has long been understood to be a major limiting factor in ICF.

Shock and blast waves are also common participants in astrophysical processes. ${ }^{10}$ In a core-collapse supernova, for example, the sudden release of an enormous amount of energy at the star's core drives a strong blast wave that propagates out through layers of progressively less dense matter. ${ }^{11,12}$ As the interfaces between these layers subsequently decelerate in the expansion fan behind the blast front, they are RT unstable. ${ }^{8}$ The potential significance of this phenomenon was realized with observations of SN1987A, when it was found that sphericallysymmetric explosion models failed to correctly predict the velocity and arrival time at the surface of heavy elements originating from the star's central regions. It has been suggested that the discrepancy results from the failure of the 1D models to account for the turbulent (the Reynolds number has been estimated ${ }^{13}$ to be of order $10^{10}$ ) RT mixing that is certainly occurring at unstable interfaces. ${ }^{11,12,14,15}$

It has been suggested that, despite the huge difference in length and time scales, scaled experiments of some astrophysical systems can be fielded on high energy density (HED) facilities ${ }^{16,17}$ such as the OMEGA laser at the University of Rochester's Laboratory for Laser Energetics (LLE) ${ }^{18}$. This follows from the fact that, provided that viscosity as well as thermal and radiation transport can be neglected, the Euler equations are scale invariant. For example, consider two different systems (but 
with matching EOS) driven by strong shocks with velocities $\mathrm{v}_{1}$ and $\mathrm{v}_{2}$. If the postshock density profiles are identical modulo a scale factor $h_{1} / h_{2}$, where $h_{1}$ and $h_{2}$ are characteristic length scales of the two systems, then the two systems will evolve identically on normalized timescales $h_{1} / v_{1}$ and $h_{2} / v_{2}$. The degree to which viscous effects are negligible is of course scale-dependent, and they cannot be ignored in the vicinity of the Kolmogorov scale. Nevertheless, the hypothesis that scaled experiments can reproduce phenomenon simulated or observed on larger scales has been demonstrated for some laminar flows. ${ }^{15,19-21}$ Similarity in the structure of turbulent flows has also been observed across a wide range of scales for systems with similar characteristics, and appears to be quite general provided each system has sufficient time and available wave-number space for the development of a broad inertial range. ${ }^{22,23}$ The Reynolds number of the two flows need not be equal as long as it is in both cases sufficiently high so that the observable scales of interest are decoupled from the dissipative scales. Specifically, Dimotakis has shown that many flows exhibit a turbulent mixing transition at a critical Reynolds number of order $10^{4}$, above which their dependence on the Re is greatly diminished. ${ }^{22}$

Such a scaling has been set out for the explosion phase of a core-collapse supernova, and experiments have been designed and conducted to begin to investigate several relevant issues in the laboratory. ${ }^{12,13,15,19}$ In this paper, we present the results of numerical simulations of a series of single- and multimode RT experiments carried out at the OMEGA facility. These are 2D planar experiments for which the gross hydro is appropriately scaled to be relevant to core-collapse supernovae such as 1987A. For supernovae, characteristic length, pressure, and density scales are of order 
$10^{11} \mathrm{~cm}, 10^{-2} \mathrm{~g} / \mathrm{cc}$, and $10 \mathrm{Mbar}$, respectively. The characteristic time scale $\mathrm{h} / \mathrm{V}(\mathrm{P} / \rho)$ is thus of order $1000 \mathrm{~s}$. In the experiment, we have $\mathrm{h} \sim 10^{2} \mathrm{um}, \rho \sim 1 \mathrm{~g} / \mathrm{cc}$, and $\mathrm{P} \sim 1$ Mbar, which can be combined in the same way to give $\tau \sim 10 \mathrm{~ns}$. The $40 \mathrm{~ns}$ experiment therefore corresponds to the first several thousand seconds of the supernova's explosion phase.

In the experiment, the Reynolds number at the unstable interface grows up to be of order $10^{5}$. This is still far below the value of $10^{10}$ estimated for supernova flows, but significantly higher than Dimotakis' suggested sufficient condition for the turbulent mixing transition $\left(\mathrm{Re}_{\mathrm{mix}} \sim 10^{4}\right) .{ }^{22}$ This suggests that the experiments are approaching a regime of true relevance to supernovae. During the course of the experiments, the evolving interface passes almost immediately through the linear phase of the RT instability and continues well into the deep nonlinear regime. The eventual goal of this line of experiments is to investigate this deep nonlinear phase, the eventual transition to turbulence, and especially the subsequent turbulent flow. This phase must be obtained for the development of an experimental test-bed that is truly representative of supernova hydrodynamics. In general, transition to turbulence is as yet poorly understood in compressible HED flows. ${ }^{10}$

We begin with brief descriptions of the experiment (more complete details of the experiments will be published separately) and the radiation-hydrodynamics code CALE ${ }^{24}$ which is used for the simulations. We then present and discuss the simulation results, beginning with checks of the 1D hydrodynamics and proceeding on to single-, two-, and eight-mode perturbations. In each case, we demonstrate good agreement between the simulations and the experimental results. Our analysis shows 
that RM and target decompression effects must be considered in order to accurately describe the perturbation growth. A buoyancy-drag model is applied to the interface in each case. The model succeeds qualitatively in predicting the single-mode spike and bubble behavior. However, despite efforts to include the effects of bubble growth and merger in the application of the model to the multimode cases, it is there at best only partially successful. We also discuss the dependence of the simulated instability evolution on the laser energy, preheat, and the equation of state model, and show that the choice of EOS can significantly affect the growth rate and interface structure. Finally, we conclude with a summary of our results.

\section{Experiment}

In the experiments, ${ }^{23} 10$ of OMEGA's beams deliver a $1 \mathrm{~ns}$ pulse of $1 / 3 \mu \mathrm{m}$ laser light at one end of a directly-driven cylindrical target (shown schematically in Fig. 2). The average laser intensity on the target is typically $6 \times 10^{14} \mathrm{~W} / \mathrm{cm}^{2}$. The laser energy is nominally $5 \mathrm{~kJ}$, but can vary by more than $1 \mathrm{~kJ}$ for experiments performed on different days.

The target consists of a $150 \mu$ m-long polyimide $\left(\mathrm{C}_{22} \mathrm{H}_{10} \mathrm{~N}_{2} \mathrm{O}_{4}\right)$ pusher/ablator section with a density of $1.41 \mathrm{~g} / \mathrm{cc}$. A single- or multi-mode $2 \mathrm{D}$ perturbation is machined into one end of the polyimide pusher, in which a $200 \mu \mathrm{m}$-wide $4.3 \%$ brominated polystyrene $\left(\mathrm{C}_{500} \mathrm{H}_{457} \mathrm{Br}_{43}\right)$ radiographic tracer is embedded. The tracer serves to minimize edge and parallax effects during target imaging via side-on $\mathrm{x}$-ray 


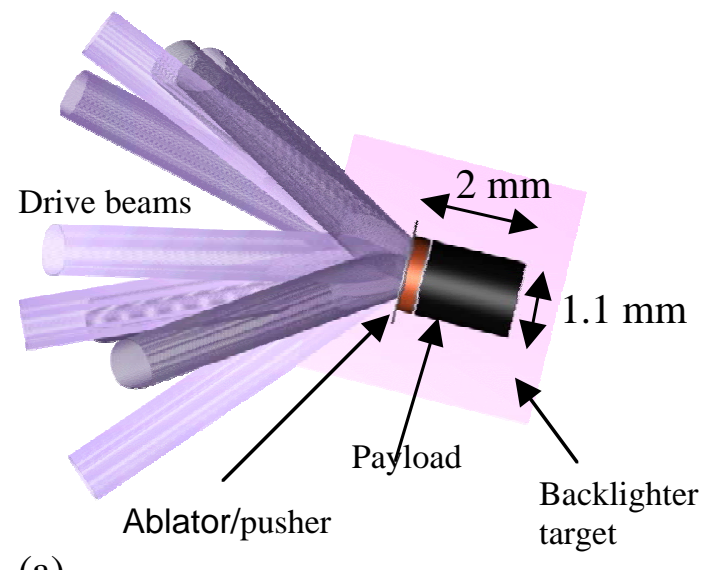

(a)

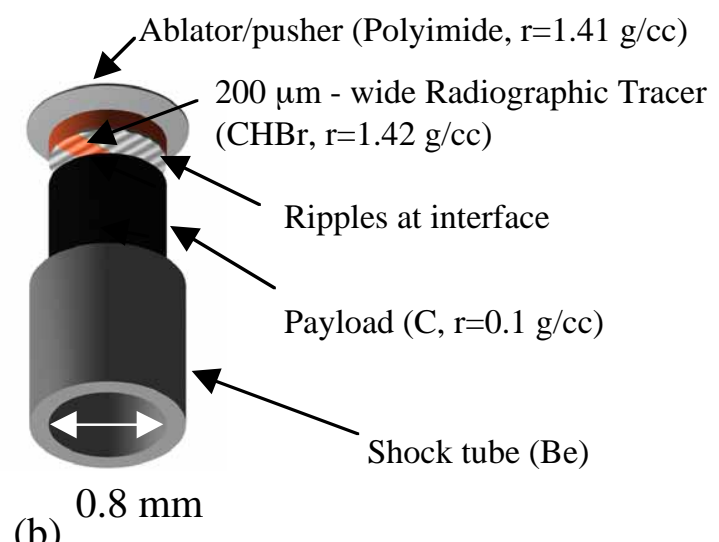

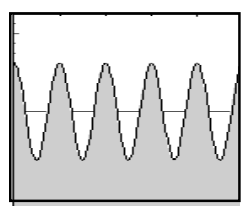

(c)

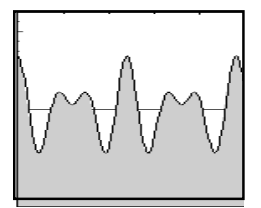

(d)

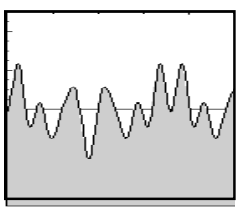

(e)

Figure 2: Target schematic showing (a) overall experiment configuration, (b) an exploded view of the target package, and imposed interfacial perturbations for (c) single-mode, (d) two-mode, and (e) eight-mode experiments.

radiography. With a density of $1.42 \mathrm{~g} / \mathrm{cc}$, the tracer is very nearly mass-matched to the pusher so as to minimize any effects of the pusher-tracer boundary on the instability evolution. A $1.9 \mathrm{~mm}$ carbon foam payload (carbonized resorcinol formaldehyde $(\mathrm{CRF})$ with density $0.1 \mathrm{~g} / \mathrm{cc}$ ) is brought into contact with the pusher, 
and the resulting assembly rests within a cylindrical beryllium shock tube with an inner radius of $400 \mu \mathrm{m}$. Because the perturbation is machined into the plastic pusher but not into the foam payload, the two materials are actually in contact only at the highest peaks of the perturbation. As a result, there exists a gap between the two materials. Since target assembly takes place in air at atmospheric pressure, the gap is initially air-filled. The extent to which that air subsequently diffuses out of the gap has not been quantified. The effect of x-ray and electron preheat on the interface has also not been measured. X-ray preheat has been evaluated by simulations, however, as discussed below.

Phase plate beam smoothing results in a supergaussian spatial profile characterized by half-width $r_{0}=412 \mu \mathrm{m}$ and order $n=4.7$. This gives fairly uniform illumination on the target within the $400 \mu \mathrm{m}$ inner radius of the shock tube. As a result, the plastic-foam interface and the transmitted shock remain nearly planer throughout the experiment.

The experiments are diagnosed via side-on and face-on x-ray radiography throughout their duration of up to $40 \mathrm{~ns}$. This is done with titanium back-lighters that produce $4.7 \mathrm{keV}$ photons for target imaging. The detector is a gated-microchannelplate-intensified x-ray camera with spatial resolution of about $10 \mu \mathrm{m}$, a gating time of about $250 \mathrm{ps}$, and a quantum efficiency of $4 \%$. The field of view is set by the backlighter spot size of about $750 \mu \mathrm{m}$. The effect of the brominated tracer layer is illustrated by considering the $\mathrm{x}$-ray transmission through the various materials. Using the densities obtained at $10 \mathrm{~ns}$ from a 1D simulation, the side-on transmission through the foam side of the interface is 0.62 . Normalized to this value, the transmission 
through the plastic side of the interface is 0.021 . Without the tracer layer, it would be 0.18. Thus, the contrast between the spike and bubble is nearly 9 times greater with the tracer layer than it would be without.

The incident laser pulse drives a strong shock wave (with Mach number $\mathrm{M} \approx$ 15) into the plastic pusher. When the laser pulse is terminated, the target begins to expand as a rarefaction wave is launched into the target. By the time the incident shock has reached the interface, the rarefaction wave has overtaken it, resulting in the formation of a blast wave. The blast wave crosses the interface at about $2.5 \mathrm{~ns}$, accelerating it to about $70 \mu \mathrm{m} / \mathrm{ns}$ and depositing vorticity that will subsequently drive Richtmyer-Meshkov ${ }^{25,26}$ (RM) growth. After passage of the blast wave, the interface begins to decelerate, and continues to do so throughout the remainder of the experiment. During the deceleration phase, the interface is RT unstable. In addition to the RM and RT instabilities, target decompression occurs during the experiment, and is responsible for about $50 \%$ of the total perturbation growth.

\section{Simulation}

We use CALE (for C-based Arbitrary Lagrangian Eulerian) to simulate the experiments. CALE is a $2 \mathrm{D}$ radiation hydrodynamics code that uses a finite-

differencing method to numerically solve the Euler equations. ${ }^{24}$ As an ALE code, it mixes elements of Eulerian and Lagrangian techniques in order to inhibit mesh entanglement. Although we generally run these simulations in ALE mode, some 
Eulerian calculations were also performed for comparison. Unless otherwise stated, planar symmetry is specified in the direction perpendicular to the computational domain (i.e. $x-y$ Cartesian rather than axisymmetric), and electron conduction is included.

The initial length of the computational grid is typically $2 \mathrm{~mm}$. In the singlemode simulations, its width is generally $25 \mu \mathrm{m}$, equal to one half the perturbation wavelength. However, simulations were also carried out with full wavelengths and multiple wavelengths. In these cases, as in the half-mode simulations, reflection boundary conditions are specified on the boundaries parallel to the target's symmetry axis, while free boundary conditions are used on the orthogonal boundaries. Finally, full-target simulations that include the shock tube were performed to verify that the large-scale hydrodynamics do not adversely affect the experiment (see Section IV.B).

Numerical radiographs can be produced by CALE and directly compared with experimental data. The numerical images, which depend on user-input x-ray opacities and the backlighter energy, provide an instantaneous snapshot of the system. Because the 250 ps gating time of the actual detector is short compared to the hydrodynamic time scales, the approximation of perfect temporal resolution is reasonable. The numerical radiographs do not account for the finite instrumental resolution or the statistical noise in the experimental radiography. We therefore always distinguish them from "simulated" $x$-ray radiographs, which can be made from the CALE results by folding in photon statistics and the pinhole resolution of the framing camera, thereby allowing for more realistic side-by-side comparisons with the data. 
The pusher density in the simulations is $1.42 \mathrm{~g} / \mathrm{cc}$ - equal to that of the brominated plastic tracer layer, and the foam density is $100 \mathrm{mg} / \mathrm{cc}$, as in the experiment. The gap between plastic and foam is included in the simulations, and its density is typically set to $1 \mathrm{mg} / \mathrm{cc}$. This should be considered an upper bound on the actual gap density, but simulations run with lower values yield virtually identical results. Although it has been shown that such a gap can have a significant effect in laser-driven RM instability experiments on the spike and bubble structure as well as the perturbation amplitude, ${ }^{27}$ simulations suggest that in these RT experiments, which involve very strong shocks and initially small-amplitude perturbations, only the amplitude is significantly affected by the presence of an air-filled gap. Omission of the gap results in a decrease of up to $10 \%$ in the amplitude and amplitude growth rate. This difference is sufficient to warrant inclusion of the gap in the simulations.

In order to investigate the dependence of the instability evolution on the choice of equation of state (EOS) model, we have run simulations with tabular EOS as well as perfect gas with various choices of adiabatic indices for the plastic and foam. We use two commonly-used sets of EOS tables, called LEOS (derived from $\mathrm{QEOS}^{28}$ ) and EOP (used, for example, in Ref. 29), which are both Thomas-Fermilike. These models do not take into account the material structure, but we do not consider this a bad approximation given that the actual foam pore size is no more than about $0.1 \mu \mathrm{m}$. As will be shown in Section IV.D, the EOS choice can nevertheless have a significant effect on the instability evolution.

The initial material temperatures in the CALE simulations discussed above are typically $25 \mathrm{meV}(290 \mathrm{~K})$. After laser deposition, radiation effects and electron 
preheat are generally not included, so the interface (along with all the pre-shock target) remains cold until the arrival of the shock. Significant levels of preheat would cause the interface to move and its perturbation structure to change prior to shock transmission. If the plastic side of the interface was heated sufficiently to cause melt, the interface would begin to move away from the laser-end of the target, possibly driving a shock into the foam, and the perturbation amplitude would decrease (remaining stable) and possibly become non-sinusoidal.

A 2D LASNEX ${ }^{30}$ calculation including radiation transport predicts that material at the interface is preheated to a temperature of about $0.4 \mathrm{eV}$ before the arrival of the shock. In order to estimate the effect this might have on the subsequent instability evolution, a simulation was run in which the initial temperature of all materials was increased to $0.4 \mathrm{eV}$ (see Fig. 3). The effect on the spike and bubble shape is relatively small, and the perturbation amplitude is reduced by about $10 \%$. At the time of shock arrival at the interface, the amplitude has been reduced from 2.5 to $1.9 \mu \mathrm{m}$, which certainly accounts for some of the subsequent growth reduction. At later times, the perturbation growth is also inhibited by the increased thermal pressure of the preheated foam. However, the LASNEX calculation predicts that the level of preheat is less than $0.2 \mathrm{eV}$ beyond about $50 \mu \mathrm{m}$ into the foam. Consequently, the resulting reduction could be considerably less than the $10 \%$ seen in the CALE calculation with unrealistically uniform $0.4 \mathrm{eV}$ preheat. Since the main effect of preheat is to reduce the initial amplitude, another simulation was run in which the initial temperature was only $25 \mathrm{meV}$ but the initial amplitude was set to $2.0 \mu \mathrm{m}$. The resulting amplitude reduction at later times was never greater than 5\%. Beyond $15 \mathrm{ns,}$ 


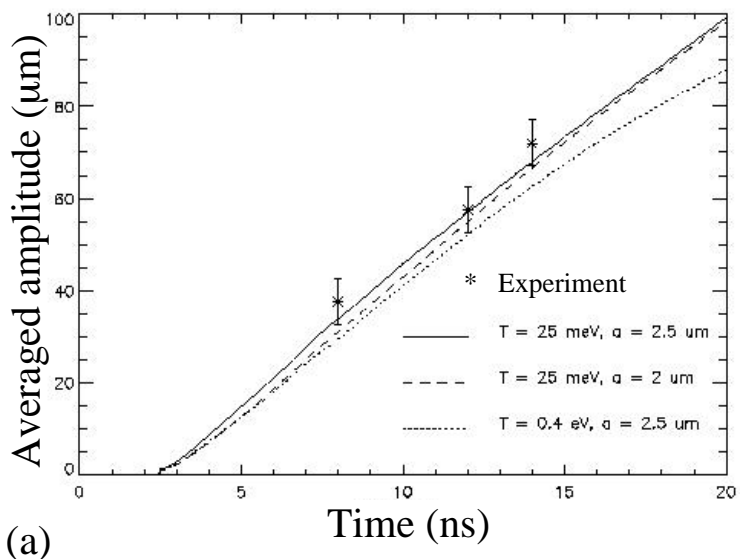

(a)

Unshocked

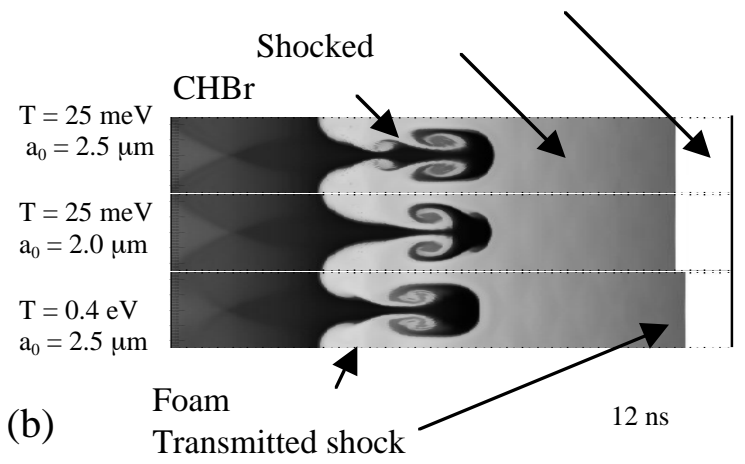

Figure 3: Estimation of the effect of preheat. LASNEX predicts that the interface is preheated to $0.4 \mathrm{eV}$ before shock refraction. (a) A CALE simulation run with the initial temperature of all materials increased from $25 \mathrm{meV}$ to $0.4 \mathrm{eV}$ yields a decreased perturbation amplitude. The pre-shock amplitude is reduced from $2.5 \mu \mathrm{m}$ to $1.9 \mu \mathrm{m}$. A third calculation with a low initial temperature but with the initial amplitude reduced to $2.0 \mu \mathrm{m}$ does not differ significantly at later times from the larger initial amplitude case. The results suggest that the effect of preheat on the amplitude is less than 5\% after shock refraction. (b) Numerical radiographs show that the effect on the shape of the spike and bubble is relatively small. All three simulations were run with EOP EOS tables for all materials. The shock is moving from left to right, the dark region on the left (including the spike) is the plastic pusher material, the lighter region to the right (including the bubble) is shocked foam, and the white region on the far right is unshocked foam.

the amplitude histories are virtually identical. Consequently, we do not consider x-ray preheat to have a significant impact on the instability evolution in these experiments. 


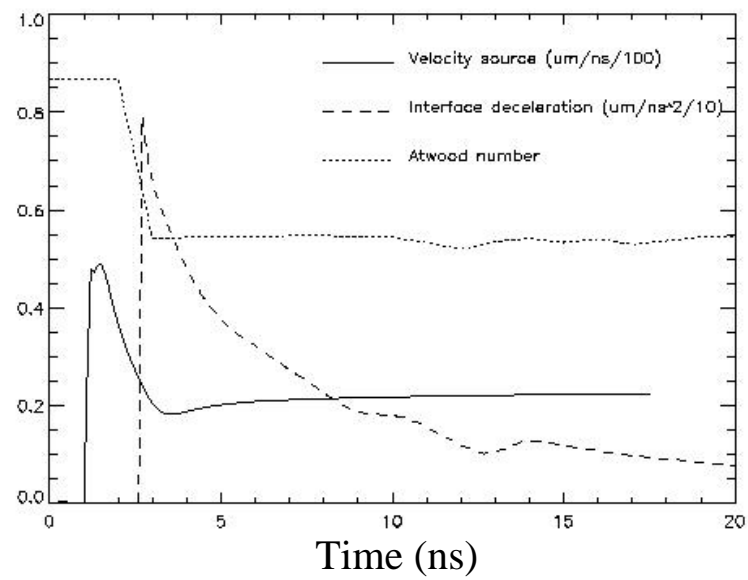

Figure 4: Velocity source: CALE simulations are driven with a time-dependent velocity source extracted from a $2 \mathrm{D}$ LASNEX simulation which in turn is driven by a $3.5 \mathrm{~kJ} 1 \mathrm{~ns}$ laser pulse. The curve shown is the velocity of the on-axis fluid element initially located $70 \mu \mathrm{m}$ into the plastic pusher $(80 \mu \mathrm{m}$ from the plastic-foam interface). The plastic-foam interface deceleration and Atwood number from a 1D CALE simulation driven by this velocity source are also shown. The post-shock Atwood number remains nearly constant at about 0.54 .

Three different methods have been used in the simulations to drive the incident shock. In the first method, a time-dependent velocity source is extracted from a 2D laser-driven LASNEX simulation and then input into CALE (see Fig. 4). This is usually done with the time-dependent pulse shape from an actual experiment, but we have found that a simple square pulse gives nearly identical results. In the first step of the second case, a 1 or 2D laser-driven LASNEX or CALE simulation is run. At the time that the laser turns off, the density, temperature, velocity, and position of the resulting shock-compressed slab is recorded. This information is then input into the full-sized 2D CALE simulation as a high-pressure, high-velocity slab, which then evolves into a blast wave and subsequently drives the instability. The velocity drive is generally used in ALE calculations, while the slab drive is more naturally 
implemented in Eulerian runs. In the third method, CALE's laser beam package is used to directly drive the simulated target with a square pulse. The three methods yield virtually identical results (the agreement among the interface trajectories is at all times better than $2 \%$ ) when the energy deposition in the laser-driven case is spatially uniform. Since this is always the case in our half-wavelength simulations, we will in those cases not specify which mechanism was used. In full-target simulations, however, the laser-drive has the advantage that it can be applied with the actual supergaussian intensity profile delivered by OMEGA, and so such cases will be presented with the drive mechanism used.

A resolution study was performed to ensure an adequate level of convergence in the simulations. In the study, the zero-time cell aspect ratio, defined as the ratio of the transverse to parallel cell dimensions, was held fixed at 5/3. Amplitude histories and numerical and simulated radiographs at 8 and 14 ns are shown in Fig. 5 from four simulations with transverse resolution ranging from 30 to 240 points per perturbation wavelength (ppw). The interface position (and therefore velocity) shows little variation from one simulation to another, and the shock position is nearly identical in all four cases. The variation in amplitude from the mean is in each case less than $6 \%$, and does appear to vary systematically with resolution. The most significant variation is in the small-scale details. Specifically, as the resolution increases, more and more Kelvin-Helmholtz $(\mathrm{KH})$ rollup appears along the interface, with an increasingly smaller minimum scale length. The variation of the late-time spike stalk on resolution is apparent in the simulated radiographs, and the calculation with the lowest 


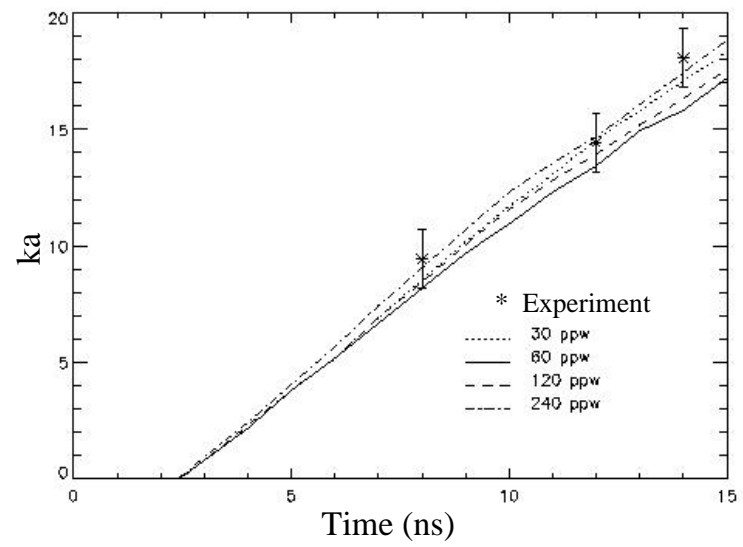

(a)
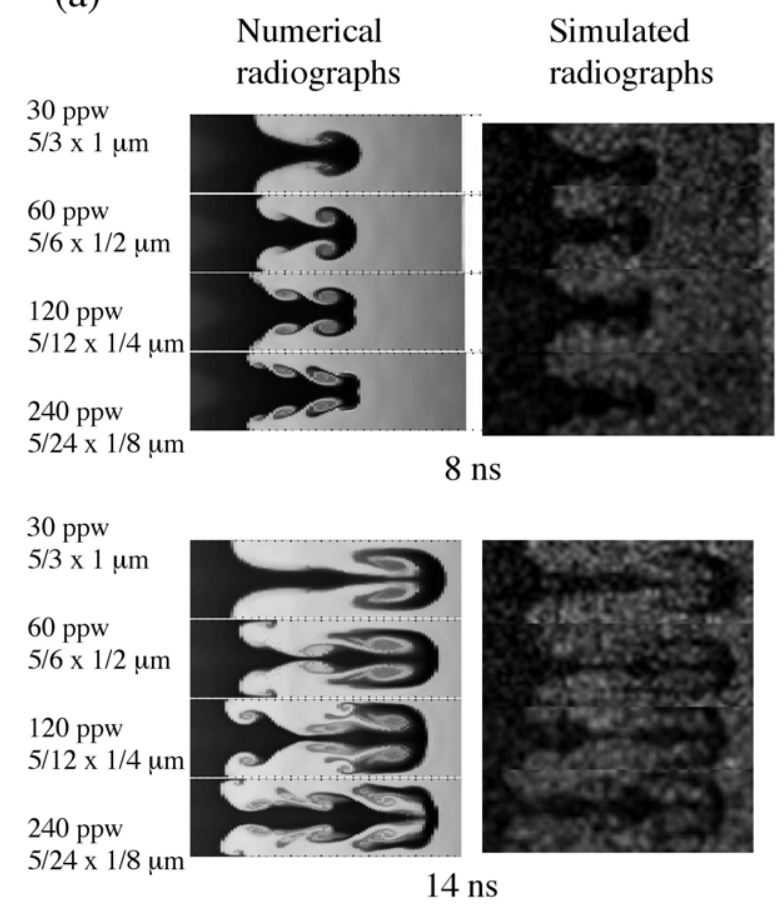

(b)

Figure 5: Resolution study. (a) Amplitude histories and (b) Numerical and simulated radiographs from CALE simulation at 8 and 14 ns. Simulated radiographs include the effects of the instrumental resolution and noise due to photon statistics. In each case, the cell dimension aspect ratio is $5 / 3$.

resolution agrees best with the data. Though the increase in $\mathrm{KH}$ rollup with resolution has rather limited impact on experimentally observable scales, the question of its 


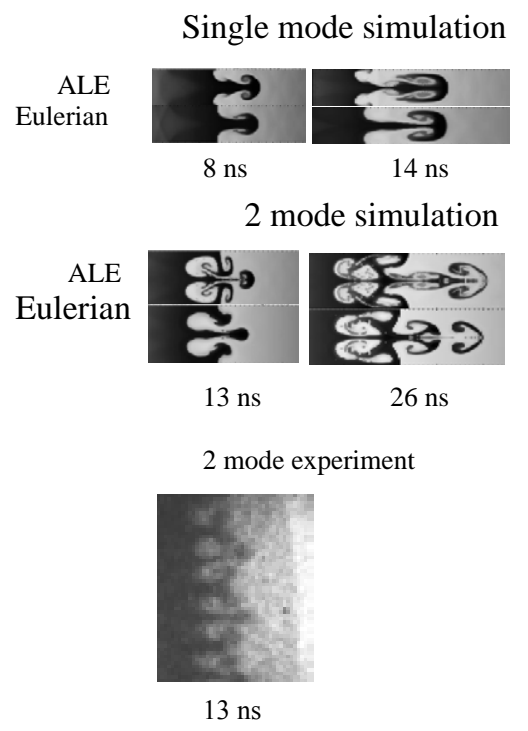

Figure 6: ALE/Eulerian comparison for single and two-mode targets. KelvinHelmholtz instability effects are more prominent when CALE is run in ALE mode than in Eulerian. In the two-mode case, the $\mathrm{T}=13 \mathrm{~ns}$ result from the Eulerian calculation seems to agree better with the data, suggesting that these effects are exaggerated in the ALE runs.

veracity should be considered in studies of the approach and transition to turbulence. Eulerian calculations exhibit less small-scale KH activity than do ALE simulations run at the same transverse resolution (see Fig. 6). In most cases, the difference is too small to be resolved by the experiment. However, experimental radiography from the two-mode experiment at $13 \mathrm{~ns}$ appears to be better reproduced by the Eulerian calculation. Though not conclusive, this suggests that the relative increase in $\mathrm{KH}$ activity is perhaps stimulated by the grid motion algorithm selected in the ALE simulations.

Half-wavelength simulations are generally run with a transverse resolution of $60 \mathrm{ppw}$. This appears to be adequate to provide a sufficient level of convergence on the experimentally observable scales. The limited numerical resolution corresponds to 
a resulting limitation in Reynolds number. With 60 ppw, the simulation Reynolds number is about 1200 when the value in the experiment is of order $10^{5}$. Thus, we do not expect the simulations to reproduce the smallest-scale features present in the experiment. Since these scales are also below the current experimental resolution, this limitation is important only if there is significant coupling between the large and small scales.

To summarize, a baseline calculation spans the entire target length and one half of one perturbation wavelength in the transverse direction with resolution of 60 ppw. It includes an air-filled gap where the corrugated plastic interface comes into contact with the planar surface of the foam payload, and is run with planar symmetry in the direction perpendicular to the computational domain. The code's ALE feature is enabled, electron conduction is included, and the initial temperature is set to 25 meV (no preheat). Finally, tabular EOS are used for all materials.

\section{$\underline{\text { IV. Simulation results }}$}

\section{A. Zero-order hydrodynamics}

A 1D simulation is used to investigate the experiment's zero-order hydrodynamics. When the EOP tables are used for all materials, the pressure and temperature in the pusher behind the incident shock are about $55 \mathrm{Mbar}$ and $25 \mathrm{eV}$, 
respectively, at the end of the $5 \mathrm{~kJ}, 1 \mathrm{~ns}$ laser pulse (45 Mbar and $20 \mathrm{eV}$ for $3.5 \mathrm{~kJ}$ pulse). Just before the shock reaches the interface, these values have fallen to about $40 \mathrm{Mbar}$ and $20 \mathrm{eV}$ (or $25 \mathrm{Mbar}$ and $16 \mathrm{eV}$ with $3.5 \mathrm{~kJ}$ drive). Figure 7 shows the time dependence of the pressure at the interface, along with the density, temperature, and sound speed on both sides of the interface. The post-shock pressure at the interface is about 4.5 Mbar just after shock transmission and falls to about 0.5 Mbar by $40 \mathrm{~ns}$. Even at late times, the shock pressures are sufficiently high that foam crush is not an issue. The pre-shock Atwood number A is 0.87, and the post-shock Atwood number $\mathrm{A}^{*}$ is nearly constant at 0.54 (see Fig 4).

The validity of the $1 \mathrm{D}$ hydrodynamics was verified by comparing the code's prediction of the shock and interface trajectories with experimental measurements, as shown in Fig. 8. The incident shock arrives at the interface rather quickly - in about $2.5 \mathrm{~ns}$, so the extent to which the comparison of the incident shock speed can be made is limited. Except in full-target simulations driven by CALE's laser package with the actual beam spatial profile, the transmitted shock speed is consistently too high for any reasonable EOS tried. The simulation results begin to deviate substantially from the data by approximately $15 \mathrm{~ns}$. The three late-time data points (one at $21 \mathrm{~ns}$ and two at $26 \mathrm{~ns}$ ) show that, after $20 \mathrm{~ns}$, the simulated shock has advanced between 120 and $200 \mu \mathrm{m}$ ahead of the actual shock position. This is between 9 and $14 \%$ of the distance traveled by the simulated shock at those times, and between 24 and $44 \%$ of the shockinterface separation. Correctly matching the shock-interface separation is important while the transmitted shock is close enough to the interface to significantly inhibit perturbation growth. This can happen either due to a shock-proximity effect, when the 


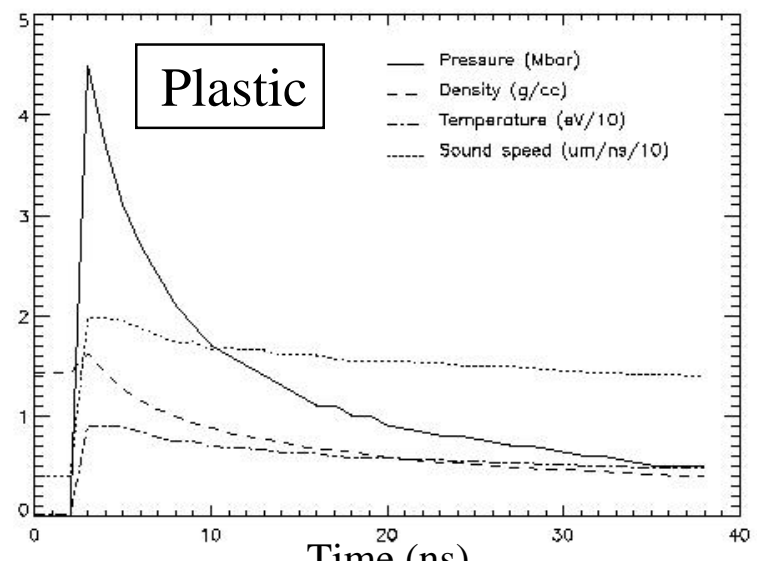

(a)

Time (ns)

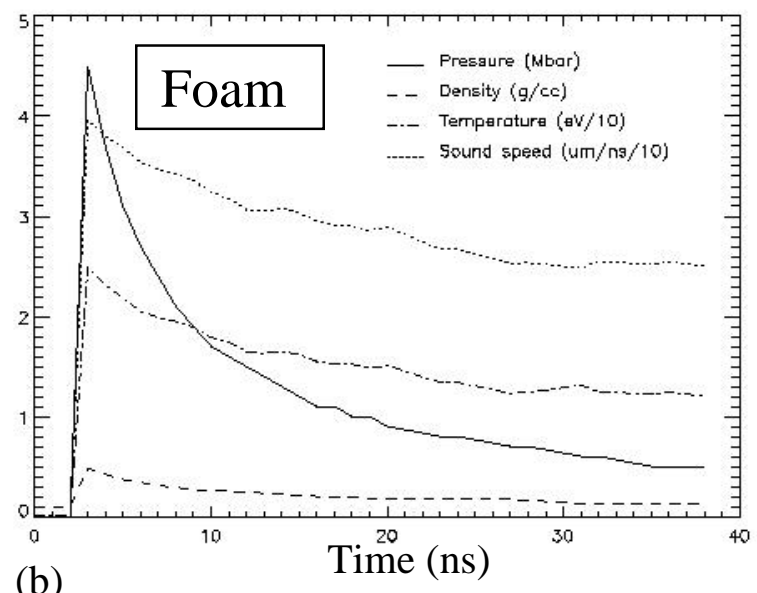

(b)

Figure 7: Time-dependence of fluid variables at the plastic-foam interface from a 1D CALE simulation: (a) Plastic side of the interface (b) Foam side of the interface. The pressure (solid line) and velocity (not shown) are continuous across the interface.

spike-shock distance is significantly less than the perturbation amplitude, or as a bounded-flow effect. In our simulations, the ratio $\left(z_{\text {shock }}-z_{\text {spike }}\right) /(2 a)$, where $z_{\text {shock }}-$ $Z_{\text {spike }}$ is the distance from the spike tip to the transmitted shock and 2a is the peak-tovalley amplitude, grows to 0.80 within $1 \mathrm{~ns}$ after shock refraction through the interface and subsequently climbs monotonically to about 1.20 . The experimental values of this ratio at 8,12 , and 14 ns are $0.91,0.71$, and 0.90 , respectively. Hecht $e t$ $a l .^{16}$ have considered the problem of a bubble rising into a fluid that is wall-bounded 

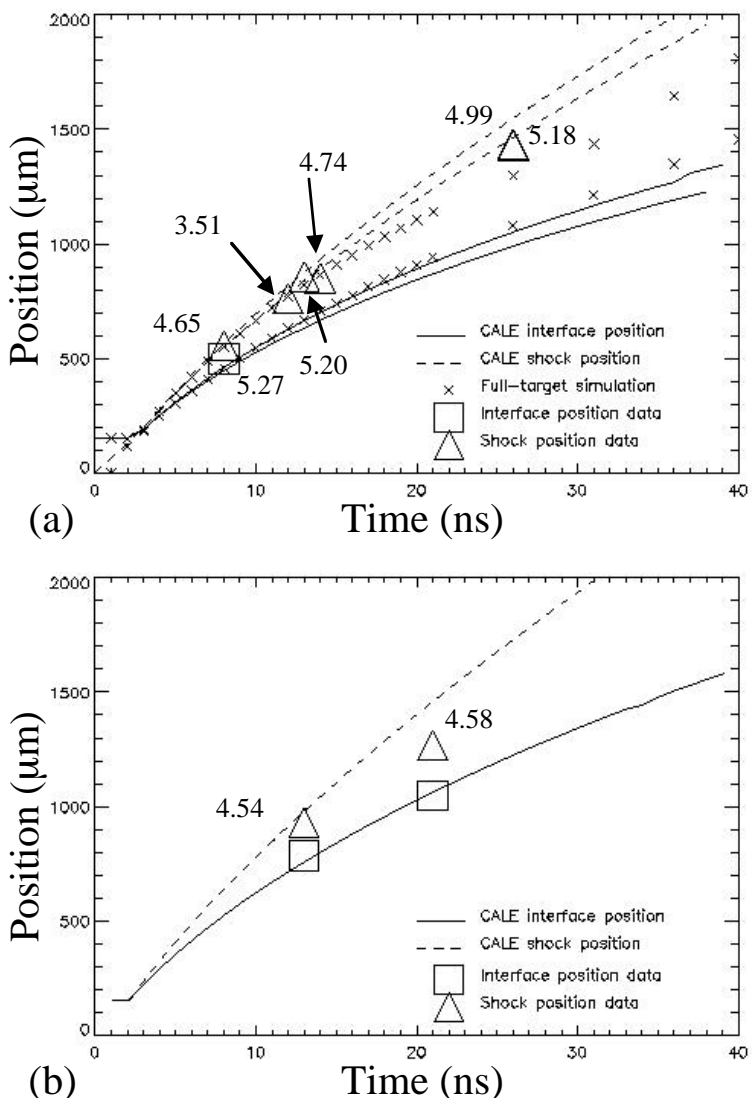

Figure 8: Interface and shock trajectories with (a) $\rho_{\text {foam }}=100 \mathrm{mg} / \mathrm{cc}$ and (b) $\rho_{\text {foam }}=50$ $\mathrm{mg} / \mathrm{cc}$. In (a), results are included from simulations with both $5.0 \mathrm{~kJ}$ drive (upper curves) and $3.5 \mathrm{~kJ}$ drive (lower curves). In (b), the drive energy is $5 \mathrm{~kJ}$. The full-target simulation includes the Be shock tube and laser spot spatial profile as well as consequent edge-effects including divergence and shock curvature. The plastic-foam interface is initially planer. All other CALE results are from 1D simulations. Experimental data is shown with points that are approximately four times the experimental error. The experimental energies (in $\mathrm{kJ}$ ) corresponding to each data point are included in the figures.

from above. They find that the inhibiting effect of the wall on the bubble velocity decays exponentially with increasing bubble-wall separation as $\mathrm{e}^{-2 \mathrm{k}(\mathrm{d}-\mathrm{z})}$, where $\mathrm{d}$ and $\mathrm{z}$ are the heights of the wall and bubble, respectively. If we assume for our case that the transmitted shock acts as a bounding wall and that spike growth suppression follows the same scaling as found for the bubble, then this effect becomes negligible when $\mathrm{z}_{\text {shock }}-\mathrm{z}_{\text {spike }} \gg{ } 4 \mu \mathrm{m}$. This condition is satisfied within about $3 \mathrm{~ns}$ in the simulations, 
and probably in about $4 \mathrm{~ns}$ in the experiment. Consequently, the shock quickly moves far enough away that its effect on the perturbation growth is small except perhaps during the first few ns. The difference between measured and simulated shock speeds nevertheless does indicate that the simulations are missing something. We believe that that there are two contributing factors. First of all, the EOS tables may underpredict the foam compressibility in some regions of pressure-volume space. This point, along with its consequences on accurate modeling of the instability, will be further discussed in Section IV.D. Secondly, the artificial planarity of the shock in the simulations also contributes to its anomalously high speed. Because of lateral expansion, the actual shock speed on the target axis is less than predicted by the 1D simulation. The full-target laser-driven simulation significantly over-predicts the shock curvature, and consequently actually under-predicts the shock speed at late times [see Fig. 8(a)]. We believe that this is due to imperfect modeling of the laser deposition. Since the shock appears nearly planer in the data, shock speed reduction due to lateral expansion is probably a lesser effect compared to the EOS.

The dependence of the zero-order hydrodynamics on the choice of EOS model used in CALE is shown in Fig. 9. In the simulations, the initial speed of the transmitted shock in the foam is about $70 \pm 3 \mu \mathrm{m} / \mathrm{ns}$, and the precise value in that range depends on both the drive energy and the EOS. The spread in results obtained with the various EOS models is comparable in magnitude to the variation caused by varying the drive energy over the range seen in the experiments. 


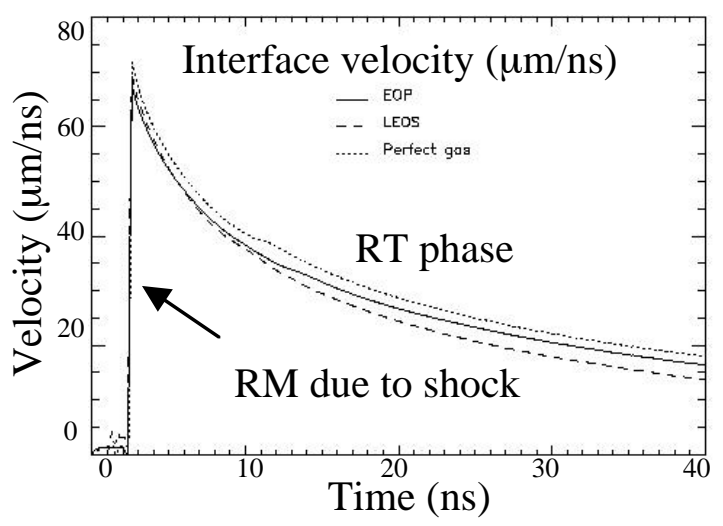

Figure 9: Interface velocity from a 1D CALE simulation driven by a $3.5 \mathrm{~kJ} 1 \mathrm{~ns}$ laser pulse. The shock induces RM growth, and the interface is RT unstable during subsequent deceleration phase.

\section{B. Single-mode perturbation}

The single-mode perturbation is characterized by a wavelength of $50 \mu \mathrm{m}$ and an amplitude of $2.5 \mu \mathrm{m}$. Side-on radiographs of the instability growth were obtained from 3 separate laser shots at 8,12, and 14 ns [see Figs. 10(a)-10(c)]. The laser energies corresponding to the data shown in Figs. 10(a)-10(c) are 4.65, 3.50, and 4.74 kJ, respectively. The CALE-produced interface trajectories in Fig. 9 are from simulations driven by a $3.5 \mathrm{~kJ}$ pulse. When a $5 \mathrm{~kJ}$ pulse is used instead, the initial post-shock interface velocity in increased by about $4 \mu \mathrm{m} / \mathrm{ns}(6 \%)$, the displacement of the interface (at $40 \mathrm{~ns}$ ) from its initial position has also increased by $6 \%$ (from 1110 to $1180 \mu \mathrm{m})$, and the perturbation amplitude at $40 \mathrm{~ns}$ has increased by about $4 \%$. At the times of the three images in Fig. 10, the absolute amplitude increase is $3-5 \mu \mathrm{m}$ (Even less when the comparison is made at identical interface position rather than time). Since this value is below the experimental resolution of about $10 \mu \mathrm{m}$, it is fair 


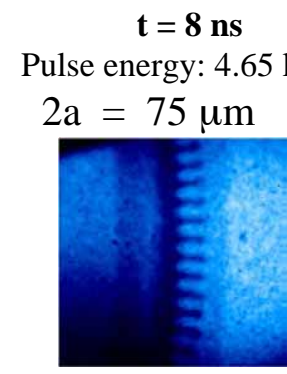

(a)

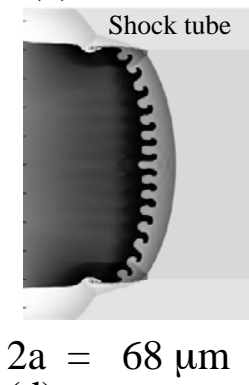

(d)

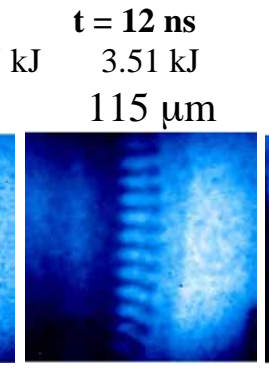

(b) $\mathbf{t}=\mathbf{1 4} \mathbf{~ n s}$

4.74

$144 \mu \mathrm{m}$

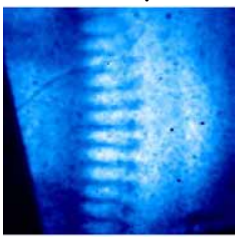

( c)

(c)

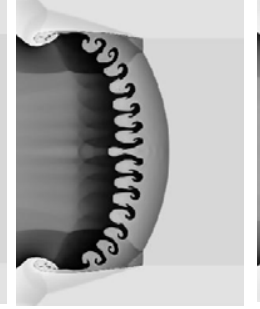

$121 \mu \mathrm{m}$

(e)

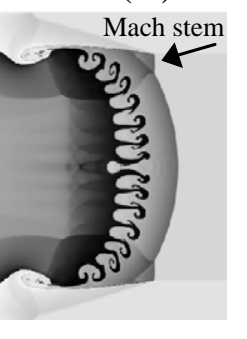

$150 \mu \mathrm{m}$

(f)

Figure 10: Single-mode data and simulation. The simulation was run in cylindrical coordinates (resulting in the unphysical interface asymmetry on axis) and driven by CALE's laser beam package with a supergaussian spatial profile characterized by half-width $\mathrm{r}_{0}=412 \mu \mathrm{m}$ and order $\mathrm{n}=4$.7. These parameters are as in the experiment.

to conclude that, with present diagnostics, the existing uniformity of laser energy is satisfactory.

Numerical radiographs at the same times, all from one full-target simulation driven by a laser pulse with the correct supergaussian spatial profile, are shown in Figs. 10(d)-10(f). The primary effect of the target-scale hydrodynamics is the curvature of the shock and interface. As noted previously, the curvature in the simulation is greater than in the experiment and, as a result, the transmitted shock speed on axis is too low at late times. There is a Mach stem apparent in the simulations near the edge of the shock-tube, but its presence in the experiment cannot be confirmed or refuted based on the radiographs available. The resolution in the full- 
target simulation (30 ppw) is only half that typically used in the half-wavelength simulation. Nevertheless, the gross features of the experiment are apparent in the simulation. In particular, the simulation reproduces the release waves originating from the shock tube walls. These waves do not reach the target axis until about $25 \mathrm{~ns}$, and so cannot affect the on-axis interface structure until very late times.

The amplitude history produced by a CALE half-wavelength simulation agrees (to within the experimental resolution of about $10 \mu \mathrm{m}$ ) with amplitude data extracted from the radiographs shown in Fig. 10 (see Fig. 11). The reason that this is true for a single simulation despite the experimental variation in drive energy is that the incident shock velocity scales as the one-third power of the drive intensity. For the data points shown, the experimental resolution is between 7 and $15 \%$ of the interpenetration-width. Thus, the apparent agreement in amplitude does not conflict with the obvious over-prediction of the transmitted shock displacement at late times. The latter is of order $10 \%$ after10 ns.

After shock transmission, the instability developing at the interface evolves almost immediately through the linear regime (in about $1 \mathrm{~ns}$ ), becomes nonlinear, and continues well into the deep nonlinear regime. The degree of nonlinearity attained in the simulation is apparent in the amplitude plot [Fig. 11(c)], which shows that ka reaches a value of about 29 at $40 \mathrm{~ns}$, as well as in the series of density plots shown in Fig. 11(e). An asymmetric spike and bubble structure, typical of the nonlinear RT and RM instabilities, has developed by $5 \mathrm{~ns}$ ( $2.5 \mathrm{~ns}$ after shock transmission). At later times, the spike becomes a long thin structure and develops prominent rollups at its tip. 

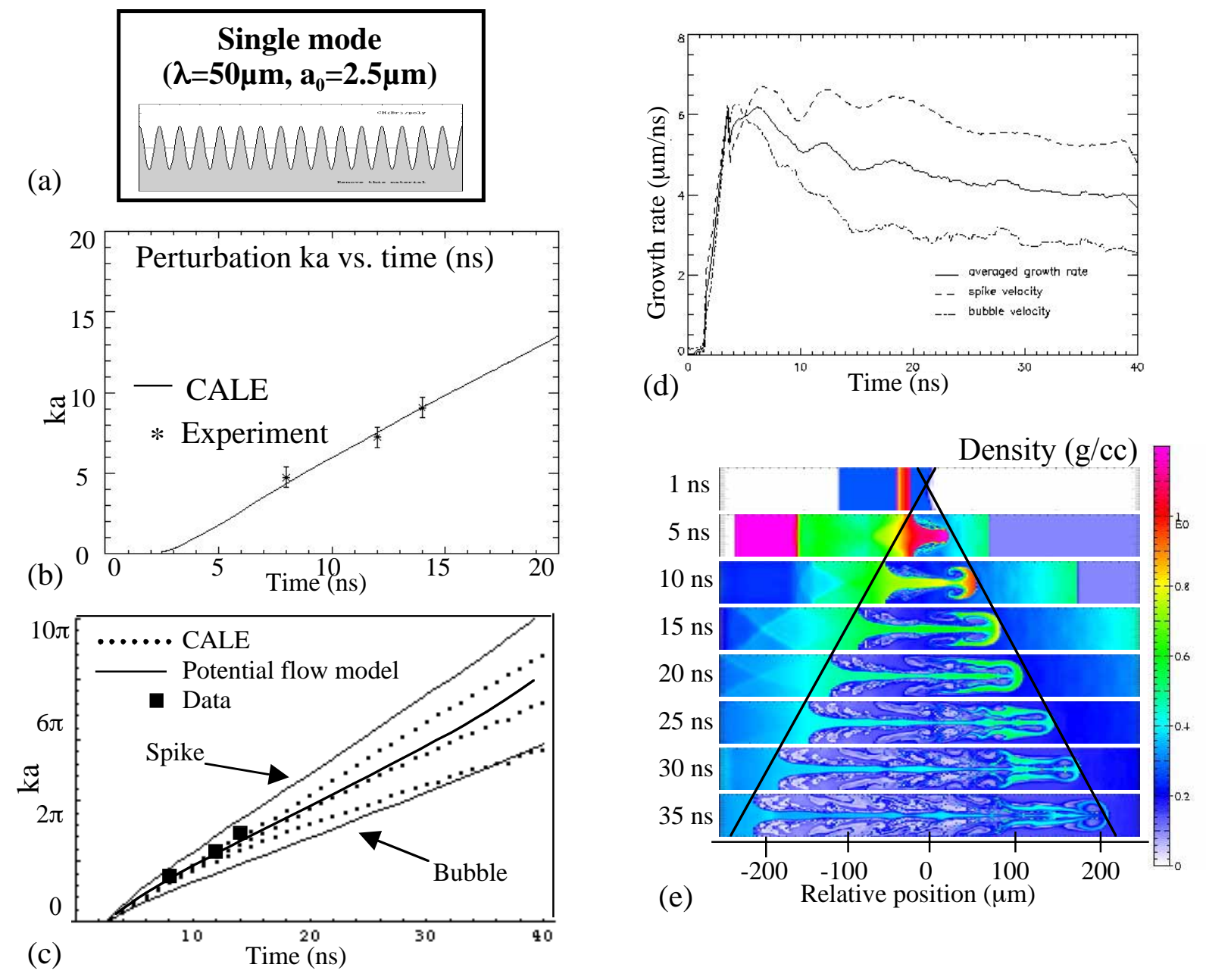

Figure 11: (a) Single mode perturbation. (b) Comparison of the simulated amplitude history with the experimental data show good agreement. (c) Comparison of the data with the experiment and Goncharov's potential flow model with decompression. (d) Spike, bubble, and averaged amplitude growth rates. (e) Density plots show the development of highly nonlinear spike and bubble structure. The simulation in (b) and (e) use LEOS for the plastic and EOP for the foam and a $3.5 \mathrm{~kJ}$ drive, while (c) and (d) use EOP for both materials and a $5 \mathrm{~kJ}$ drive. 
In the nonlinear phase, the amplitude growth is approximately linear in time despite the fact that the deceleration at the interface, and consequently the RT growth rate, is approaching zero (see Fig. 4). This behavior can be attributed to the decompression of the target in the expansion fan behind the shock front. Most nonlinear RT models are incompressible, and therefore do include perturbation growth resulting from target decompression. However, Goncharov has developed a potential flow model with a time-dependent density profile. ${ }^{31}$ The model captures the general behavior of the perturbation, but typically overpredicts the spike growth and underpredicts the bubble growth [see Fig. 11(c)]. In order to compare the simulation results with models of the RT instability that do not include material expansion, we must first subtract the expansion contribution from the spike and bubble amplitude histories. Separate spike and bubble amplitudes are obtained by simply subtracting the interface position, predicted by a 1D simulation, from the spike and bubble positions. The time-dependent fluid velocities at the spike and bubble positions are extracted from the same 1D simulation, and are interpreted as the spike and bubble expansion velocity histories. Integration of these functions yields the expansion amplitude histories, which are then subtracted from the actual amplitude histories to obtain the expansion-corrected RT spike and bubble amplitudes. Even after subtraction of the decompression effect, the spike and bubble amplitudes grow up to values of $\mathrm{ka} \approx 13$ and $\mathrm{ka} \approx 9$, respectively. In Fig. 12, the decompression-corrected amplitude curves are compared to the prediction of the buoyancy-drag model of Oron et al..$^{32}$ The model, which follows earlier work by Hanson et al..$^{33}$ and Dimonte, ${ }^{34}$ predicts that $2 \mathrm{D}$ bubbles "rise" with a velocity determined by 


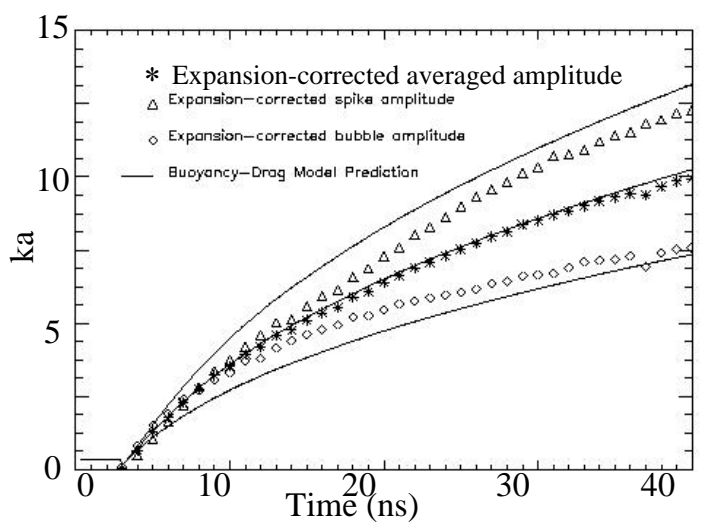

Figure 12: Comparison of the simulation results with the buoyancy-drag model of D. Oron et al. [Phys. Plasmas 8, 2883 (2001)]. The simulation is driven by a $3.5 \mathrm{~kJ}$ pulse and uses EOP for all materials

$$
\left(\rho_{b}+2 \rho_{s}\right) \frac{d u_{b}(t)}{d t}=\left(\rho_{s}-\rho_{b}\right) g(t)-\frac{6 \pi}{\lambda} \rho_{s} u_{b}^{2}
$$

The equation for the spike velocity is obtained by simply interchanging spike and bubble densities. For 3D spikes and bubbles, the numerical coefficients in Eq. 2 change from two to one and from six to two. The initial span-wise symmetry might eventually be broken in the experiments, but the simulations of course remain 2D. Consequently, the 2D coefficients remain the valid choice for comparison with the simulation at all times. As noted by Dimonte, ${ }^{36}$ such models tend to overestimate the spike-bubble asymmetry. This results from their application at early times when the perturbation has not yet reached its asymptotic state. In our case, it could also result from our approximate treatment of the RM effects, which dominate the instability growth early on. In any event, the averaged amplitude is nevertheless well predicted by the model throughout the simulation and well into the deep nonlinear regime. In addition, Eq. 2 gives a qualitatively correct description of the spike and bubble behavior. At late times, the spike and bubble velocities decay along with the driving 
interface acceleration, with the asymptotic spike amplitude significantly higher than that of the bubble.

\section{C. $R M$ contribution}

In applying the buoyancy-drag model to the simulation results, the effect of the RM instability was included by initializing the spike and bubble velocities with the Meyer-Blewett velocity. ${ }^{35}$ The Meyer-Blewett velocity is essentially Richtmyer's original impulsive model, ${ }^{25}$ given by

$$
\mathrm{v}_{\mathrm{MB}}=\mathrm{k} \mathrm{a}^{*} \mathrm{~A}^{*} \mathrm{u}_{\mathrm{i}},
$$

adapted to the case of a shock passing from a heavier to a lighter fluid. This is done by simply replacing the post-shock perturbation amplitude a* in the Richtmyer formula with the average of the pre- and post-shock amplitudes. In either case, $A^{*}$ is the post-shock Atwood number and $\mathrm{u}_{\mathrm{i}}$ is the velocity increase of the interface upon shock transmission. Evaluation of Eq. 3 for the simulation gives $\mathrm{v}_{\mathrm{MB}}=6 \mu \mathrm{m} / \mathrm{ns}$. Interestingly, this is precisely the early-time peak amplitude growth rate seen in Fig. 11(d), suggesting that the perturbation growth is dominated by the RM instability during the first couple of nanoseconds.

In order to make some estimate of the RM contribution at all times, we ran a simulation in which a modified target was driven by a constant velocity $(41.5 \mu \mathrm{m} / \mathrm{ns})$ piston. The piston velocity and pusher thickness were chosen such that the resulting long, steady shock would accelerate the interface up to the same velocity as that provided by the blast wave, and then maintain that same velocity without deceleration 
[see Fig. 13(a)]. The amplitude and velocity histories [Figs. 13(b)-13(c)] display classical RM behavior. The amplitude growth rate quickly climbs to a value that is reasonably well predicted by the Meyer-Blewett formula, and then decays while undergoing oscillations. Comparison with the amplitude growth rate observed in the RT simulation shows that the RM contribution, while initially responsible for virtually all the perturbation growth, falls to half the total after about $3 \mathrm{~ns}$ and becomes relatively insignificant shortly thereafter. This is not because the RT amplitude growth rate, which is proportional to perturbation amplitude in the linear regime, has grown much larger than the Meyer-Blewett velocity. Indeed, the total amplitude growth rate never exceeds the Meyer-Blewett velocity. Rather, it results from the relatively quick decay of the RM driving mechanism. At $30 \mathrm{~ns}$, the spike and bubble amplitudes in the pure RM simulation are roughly $50 \%$ of their decompression-corrected counterparts in the actual experiment. Despite its relatively low contribution to the growth rate during most of the experiment, therefore, the RM instability may contribute up to about half of the decompression-corrected perturbation growth [see Fig. 13(c)]. An estimate of the RM contribution obtained in this way should be considered as an upper bound, since the effects of the two instabilities do not necessarily add up linearly. Such uncertainty does not exist in the consideration of the combined contribution of the RT and RM instabilities relative to the total growth including decompression. The RT $+\mathrm{RM}$ contribution begins at $100 \%$, falls to $50 \%$ at about $20 \mathrm{~ns}$, is between 45 and $50 \%$ at $30 \mathrm{~ns}$, and continues to diminish at still later times. 

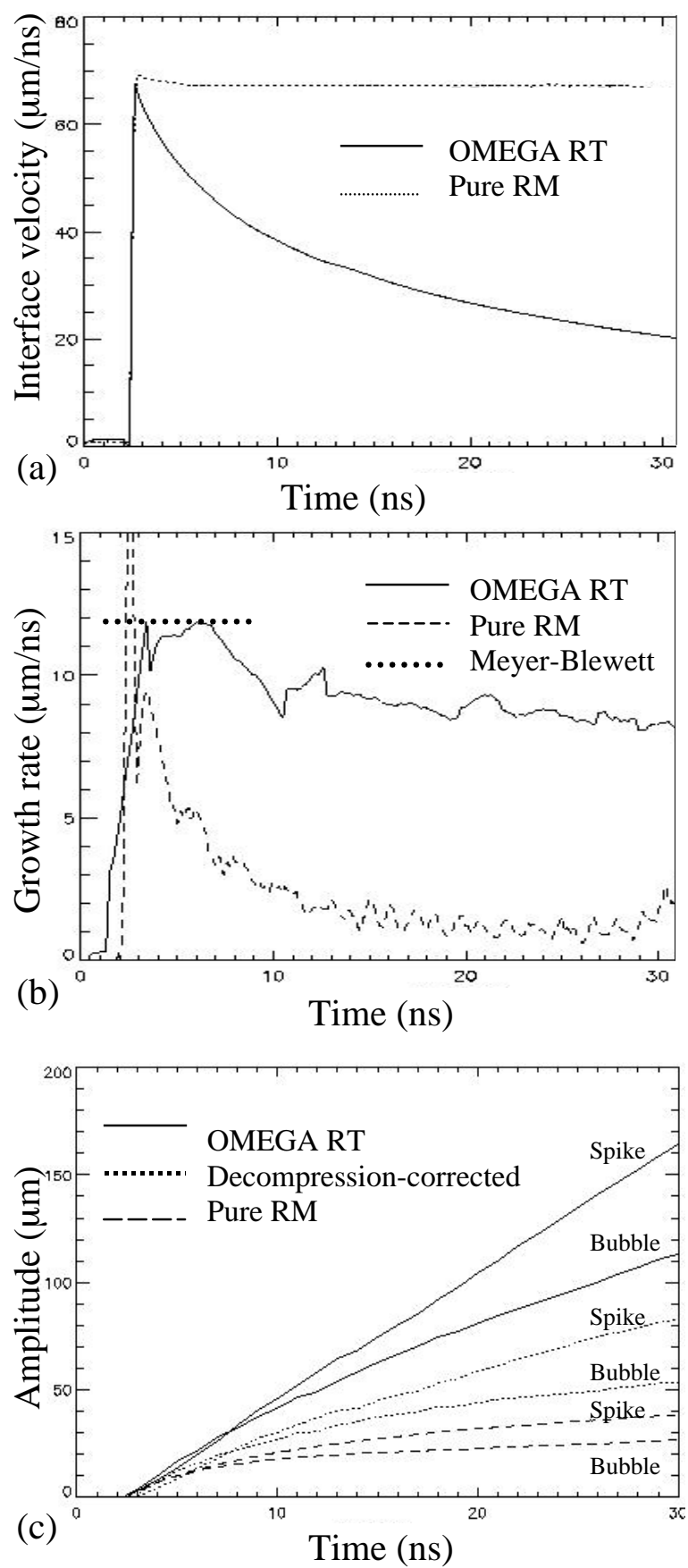

Figure 13: Estimation of contribution from RM instability and decompression. (a) Interface velocities resulting from OMEGA RT (3.5 kJ) and pure RM (24 ns 41.5 $\mu \mathrm{m} / \mathrm{ns}$ ) drives. Pure RM (b) amplitude growth rate shows classic RM behavior. The peak amplitude growth rate is well-predicted by the Meyer-Blewett velocity of 6 $\mu \mathrm{m} / \mathrm{ns}$. RT dominates over RM after a few ns. (c) Spike and bubble amplitude histories from the simulation of the experiment, expansion corrected RT + RM simulation, and the pure RM simulation. Decompression accounts for about $50 \%$ of the total perturbation growth at late times, and RM accounts for up to $50 \%$ of the 
expansion-corrected perturbation growth.

\section{Equation of state}

The simulation results are sensitive to the choice of EOS model specified in CALE. This sensitivity was investigated through a series of simulations involving different EOS combinations. The study included the EOP and LEOS tables as well as perfect gas models with a range of choices for the adiabatic indices $\gamma$ of the plastic pusher and foam payload. Relevant portions of the EOP and LEOS shock Hugoniot curves for the plastic pusher and foam payload are shown in Fig. 14. In regions of the curves accessed by the incident and transmitted shocks, EOP predicts greater material compressibility than does LEOS. This is particularly true for the foam, where the resulting limiting compression factor with EOP is about $30 \%$ greater than with LEOS.

The choice of EOS affects the interface and shock velocities, perturbation amplitude, and the spike and bubble shape (see Figs. 15-16). However, the experimental resolution is insufficient to distinguish between EOS models based on their predictions of the spike and bubble shape. The perturbation amplitude is reasonably well predicted by several of the models, which on average vary from the data by about $10 \%$, but the combination of LEOS for the plastic and EOP for the foam does the best job. The growth rate decreases as the foam compressibility increases, and the data are inconsistent with foam modeled as an ideal gas with adiabatic index less than 1.4. The transmitted shock speed is over-predicted by halfwavelength simulations using both the EOP and LEOS tables. As noted previously, 
this is partially due to lateral expansion behind the curved shock front that is not present in the half-wavelength simulations. However, this effect is small because of
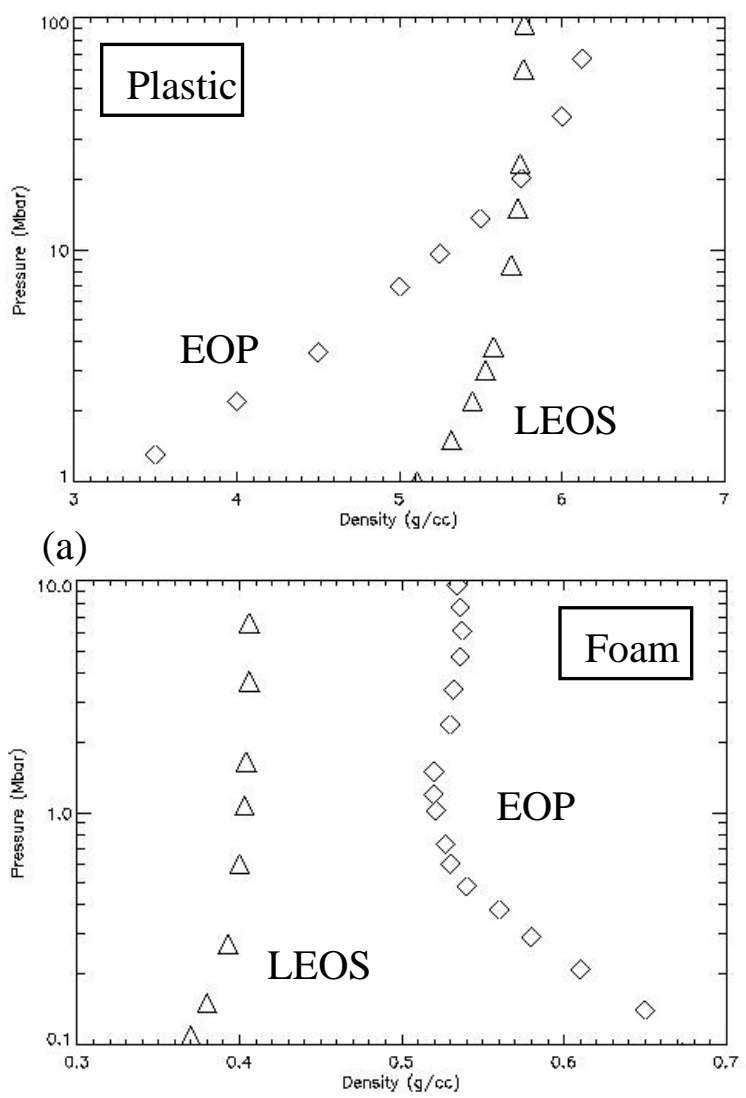

(b)

Figure 14: Tabular EOS shock Hugoniot curves in relevant ranges for (a) brominated polystyrene (used for plastic pusher) with $\rho_{0}=1.42 \mathrm{~g} / \mathrm{cc}, \mathrm{T}_{0}=25 \mathrm{meV}$ and (b) polystyrene (used for foam payload) with $\rho_{0}=0.1 \mathrm{~g} / \mathrm{cc}, \mathrm{T}_{0}=25 \mathrm{meV}$. In both cases, EOP is more compressible than LEOS at very high pressures. The difference is particularly pronounced for the foam. 


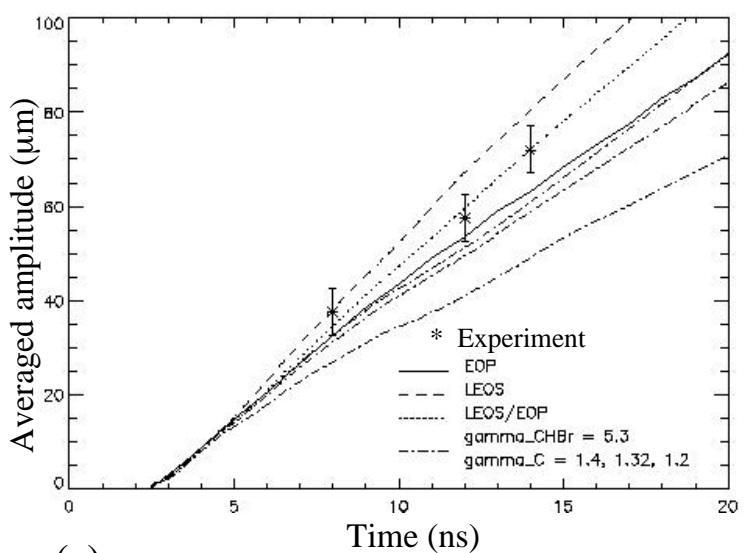

(a)

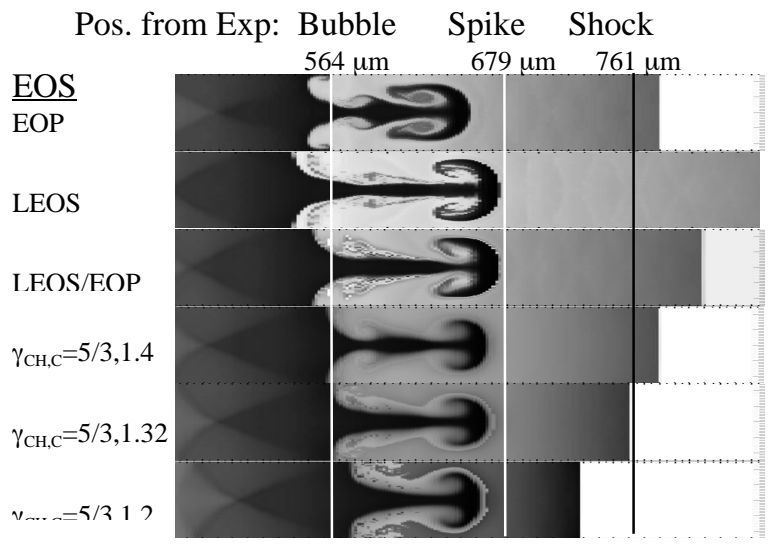

(b)

Figure 15: EOS sensitivity. (a) Amplitude histories from CALE simulations run with several different EOS combinations. For perfect gas models, the amplitude increases with decreasing foam compressibility. (b) Numerical radiographs at $12 \mathrm{~ns}$. The combination of LEOS for the plastic and EOP for the foam provides the best agreement with the data. Simulations run with either LEOS or EOP over-predict the transmitted shock speed. 


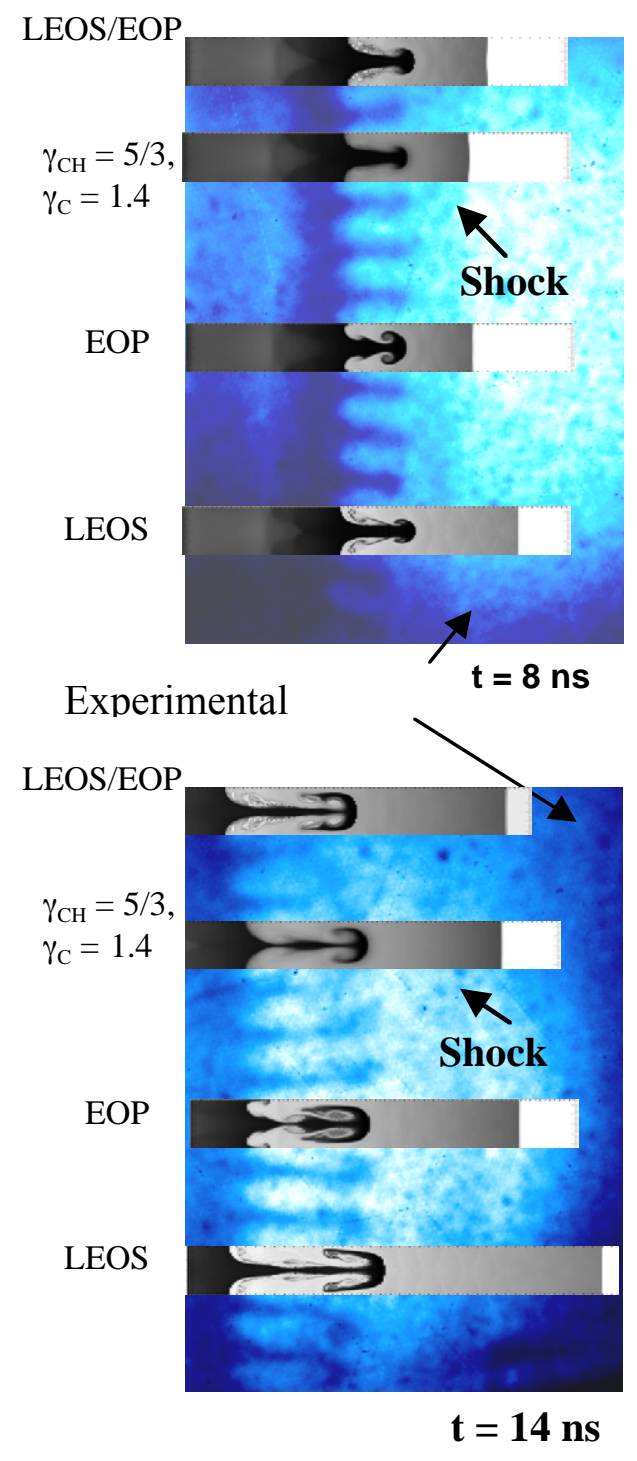

Figure 16: EOS. Choosing a good EOS model is important for matching the experiment. LEOS for the plastic with EOP for the foam is best at getting the perturbation amplitude. Perfect gas with $\gamma_{\text {plastic }}=5 / 3, \gamma_{\text {foam }}=1.4$ is best at getting the shock position relative to the interface, while perfect gas with $\gamma_{\text {plastic }}=5 / 3, \gamma_{\text {foam }}=1.32$ is best at getting the absolute shock position. Differences in spike and bubble shape are apparent in the simulations, but the data are not good enough to distinguish between the models on this basis. 
the front's large radius of curvature, and in any event does not explain the EOS model dependence seen in simulations. The LEOS tables in particular appear to greatly overstate the foam stiffness. This tendency has been noted in simulations of OMEGA $\mathrm{RM}$ experiments, ${ }^{27}$ but the discrepancy here is greater than that seen previously.

It is possible to match one or another of the key observable parameters by resorting to a perfect gas model and adjusting the adiabatic indices, but this is usually at the expense of the agreement of some other parameter. For example, we can reduce the transmitted shock speed by reducing $\gamma_{\text {foam }}$, thereby increasing the compressibility of the foam. But the resulting amplitude is soon reduced clearly below that seen in the experiment. At the same time, the interface speed becomes too high. Clearly (and not surprisingly), the materials in question do not behave as ideal gases at the energy densities accessed in these experiments and simulations.

Considering together the dependence of the perturbation amplitude history and the interface and shock trajectories found in the single-mode simulations, it seems that the target materials are best represented by LEOS tables for the plastic pusher and EOP tables for the carbon foam payload. Similar comparisons made with simulations of the multimode experiments lead to the same conclusion. Consequently, the multimode simulations presented in the next sections all use this same combination.

\section{E. Multimode perturbations}

Having demonstrated the ability to accurately simulate and model the unstable evolution of single-mode driven by a strong blast wave, we now turn to the evolution 
of multimode interfaces. Ultimately, we wish to understand the growth of highly turbulent mixing layers present within supernovae. Rather than beginning with a broad spectrum, we first consider interfaces that are initially two and eight-mode in order to investigate the approach and transition to turbulence. This will lay the groundwork for later studies with many initial modes on a wider range of scales.

\section{Two-mode}

The 2-mode perturbation [see Fig. 17(a)] is characterized by a sum of two sinusoidal components (in phase) with wavelengths of 40 and $60 \mu \mathrm{m}$ and amplitudes of 1.25 and $1.5 \mu \mathrm{m}$, respectively. This results in an initial perturbation width that is very near to the single-mode target value of $5 \mu \mathrm{m}$.

As is apparent from the simulated radiograph in Fig. 17b, the interface structure seen in the 2-mode CALE simulation at $13 \mathrm{~ns}$ is in strikingly good agreement with the data. The simulation interpenetration width at this time was 122 $\mu \mathrm{m}$, in agreement with the measured value $130 \pm 10 \mu \mathrm{m}$. The code prediction at $26 \mathrm{~ns}$ [see Fig. 17(c)] appears to be fairly good as well, but is complicated by the degradation in contrast by that time. It is quite possible that $3 \mathrm{D}$ effects have begun to appear in the experiment, marking close approach to the transition to turbulence and the limitations of the 2D simulation. The simulated mix width, $307 \mu \mathrm{m}$, is slightly larger than the observed value of $294 \pm 10 \mu \mathrm{m}$. It is clear from both images that, as in the single-mode simulations, the code over-predicts the transmitted shock speed.

The qualitative success of the buoyancy-drag model in predicting the singlemode spike and bubble growth is not apparent when the model is applied to the two- 

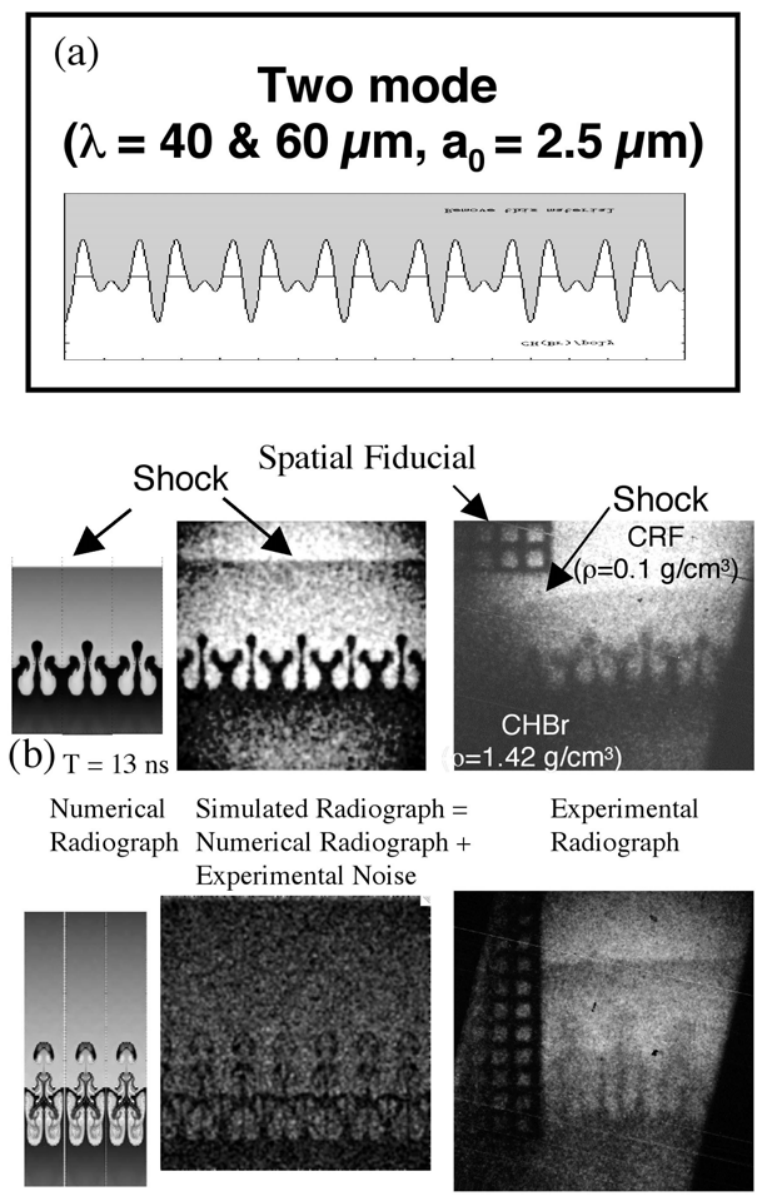

(c)

$\mathrm{T}=26 \mathrm{~ns}$

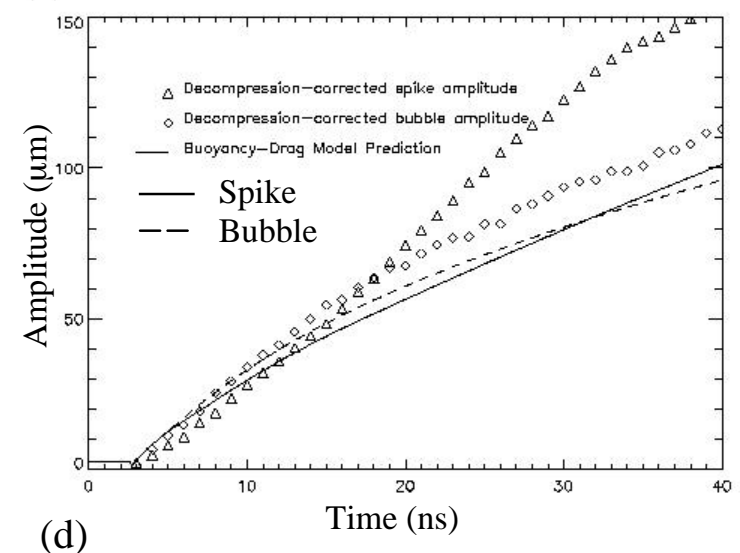

Figure 17: Two-mode perturbation simulation and data. (a) Imposed interface perturbation (b) Again, the spike and bubble size and structure look good, but the transmitted shock speed is clearly too high. (c) Comparison of decompressioncorrected simulation results with the buoyancy-drag-model prediction shows only early-time agreement. 
mode interface [see Fig. 17(d)]. In applying the model to the spike growth, the timedependent transverse width of the outlying spike was used in place of the wavelength in Eq. 2. In the case of the bubble growth, this method would lead to a gross underprediction of the observed growth at all times. Instead, the model is initialized at the largest wavelength in the problem. After the first (and only) bubble merger event occurs at about $6 \mathrm{~ns}$, this wavelength is replaced by the largest transverse scale possible in the simulation - twice the $60 \mu \mathrm{m}$ box size. The resulting model-predicted behavior of the spike and bubble is similar to that observed in the single-mode case. That is, the spike and bubble velocities begin large and decay smoothly in time. In this case, however, the spike and bubble are predicted to grow nearly symmetrically. In the simulation, the bubble growth is consistent with the model description. The spike velocity, on the other hand, abruptly increases at about $15 \mathrm{~ns}$ and subsequently undergoes a period of growth that is nearly linear in time. This fact, coupled with the agreement between the simulation and experiment, suggests the significant influence of compressibility or some other phenomenon outside the model's range of validity.

\section{Eight-mode}

The initial perturbation in the 8-mode targets [see Fig. 18(a)] is defined by the sum

$$
a(r)=\sum_{i=1}^{8} a_{i} \cos \left(\frac{2 \pi r}{\lambda_{i}}\right),
$$



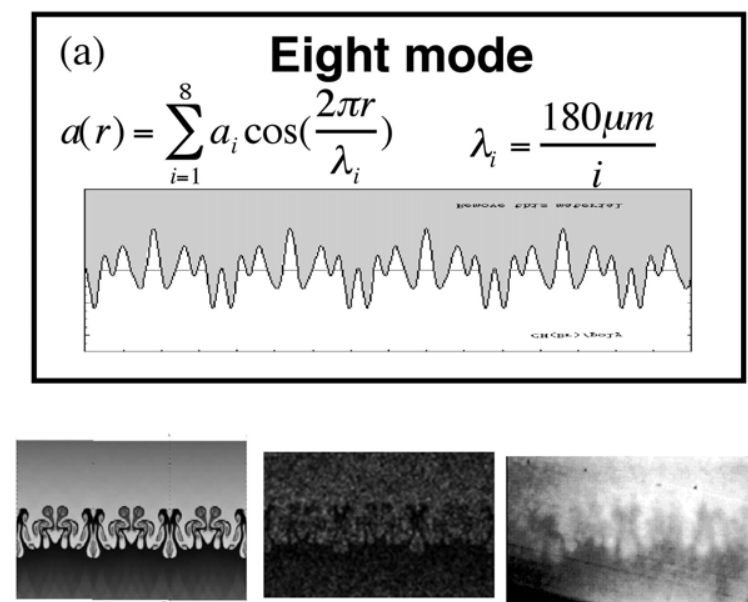

(b)

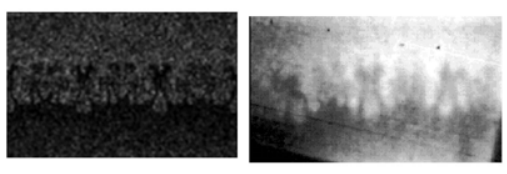

Simulated Radiograph $=$

Numerical Radiograph + Experimental

Numerical

Radiograph

Experimental Noise

Radiograph
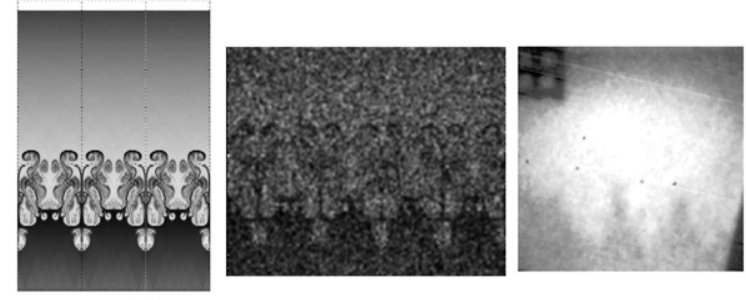

(c)

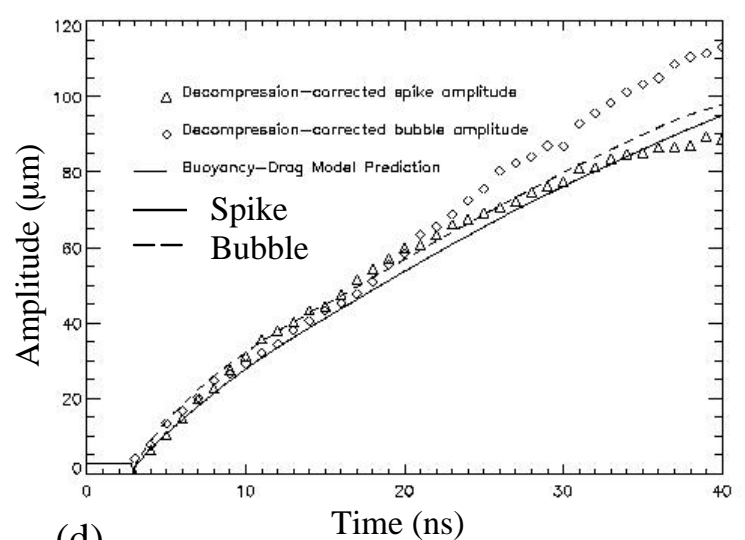

(d)

Figure 18: Eight-mode perturbation simulation and data. (a) Imposed interface perturbation. (b) CALE results are qualitatively in good agreement with the 8-mode data. (c) Comparison of decompression-corrected simulation results with the buoyancy-drag-model prediction shows only early-time agreement. 
where the wavelength vector is given by $\lambda_{i}=180 \mu \mathrm{m} / i$ and the components of the amplitude vector range in absolute value between 0.4 and $0.7 \mu \mathrm{m}$. The initial perturbation width is $4.9 \mu \mathrm{m}$ - again very close to the single-mode case.

As in the 2-mode case, the simulation results at $13 \mathrm{~ns}$ reproduce most of the features seen in the experiment, so that the simulation is in good qualitative agreement with the data on a range of scales from the largest down to the experimental resolution [See Fig.18(b)]. The simulation interpenetration width at this time is $134 \mu \mathrm{m}$, somewhat larger than the observed value of $120 \pm 10 \mu \mathrm{m}$.

Comparison with the 26 ns data is even more problematic than was the case with the 2-mode perturbation [Fig. 18(c)]. The numerical radiograph displays a dominant 180 $\mu \mathrm{m}$ mode on top of which is overlaid a feathery smaller-scale spike and bubble structure. The same dominant mode is apparent in the data, but the feathery structure is not. In fact, nothing at all is seen to protrude clearly out beyond the dominant mode. This could simply result from a loss of contrast associated with decreasing density and the appearance of 3D effects. But it could also indicate that the smallerscale structure has already transitioned to turbulent flow following the generation of initially laminar 3D structure. Considering both the Reynolds number of the flow and the time over which it is maintained in these experiments, the evolving interface could in fact undergo a turbulent mixing transition at as early as about $17 \mathrm{~ns} .^{23}$ The experimental images of the two-mode interface also show a significant loss of contrast between 13 and $26 \mathrm{~ns}$, though not so drastic as in the 8-mode case. While 
these data are suggestive, more experiments will be necessary before a firm conclusion about transition can be made. These experiments may require greater resolution and contrast between spike and bubble material, and should eventually explore the dependence on initial dimensionality and modal spectrum.

Our application of the buoyancy-drag model to the 8-mode case is shown in Fig. 18(d). In contrast with the 2-mode case, it is now the bubble front that exhibits anomalous behavior. The bubble's amplitude and velocity are both considerably greater than those of the spike after about 20 ns. Despite our efforts to account for bubble expansion and merger, we were again unable to show even qualitative agreement of the model with the simulation.

\section{Conclusions}

We have presented the results of 2D CALE simulations of blast-wave-driven hydro experiments performed at OMEGA. Simulations of single-, two-, and eightmode targets are in reasonable agreement with experiments on a range of scales from large down to the experimental resolution. In addition, the single mode evolution is well predicted by a buoyancy-drag model when the effects of target decompression are first subtracted away. We were unable, however, to use the model to correctly describe the evolution of the multimode spike and bubble fronts even when bubble expansion and merger were accounted for. The partial success of the model will be further discussed and explained in a later paper. 
Eulerian calculations appear to better reproduce the spike and bubble shapes than do the ALE runs, but the comparison is limited by the current resolution and the effect on the perturbation amplitude is negligible. The dependence of the instability evolution on the laser energy and pulse shape, x-ray preheat, and the EOS model have also been considered. The first three factors affect the amplitude at or below the 5\% level, but the choice of EOS, from commonly used tables, can significantly affect both the amplitude growth rate (at about the 10\% level) and the interface structure. All of these factors must be given careful attention in detail-sensitive simulations of experiments.

Analysis of the simulation data suggests that the RT and RM instabilities contribute roughly equally to the decompression-corrected perturbation growth, with RM dominant in the first few ns and RT dominant at later times. Together, the two instabilities account for about $50 \%$ of the total growth, with the remaining half resulting from target decompression. Consequently, all three effects must be considered in order to accurately describe the perturbation growth.

Because of limited computational resources, the simulation Reynolds number is two orders of magnitude smaller than in the experiment (currently $\operatorname{Re}^{\mathrm{CALE}} \sim 1200$ when $\left.\mathrm{Re}^{\text {exp. }} \sim 10^{5}\right)$. The hypothesized critical Re for the mixing transition lies in between these two values. The inherent 2D nature of the calculations provides an even more important limitation on their ability to reproduce all aspects of the latetime instability evolution. Consequently, the simulations cannot reproduce the smallest-scale features present in the experiment. If we consider structures computationally unresolved if their spatial scale is less than ten grid cells, then all 
unresolved scales are below the current experimental resolution. Therefore, the limited range of scales present in the simulations is important only when there is significant coupling between the large and small scales. Good agreement between data and simulations indicate that this is not the case until sometime after $14 \mathrm{~ns}$.

Having demonstrated the ability to accurately simulate the late nonlinear stages of the instability evolution for both single- and multimode perturbations, we can now with some degree of confidence proceed to model data taken at later times, as the instability approaches and, hopefully, passes through the transition to turbulence. We can also move to 3D calculations to investigate when and how the $2 \mathrm{D}$ symmetry of the experiments is broken. Since the simulations cannot reach the high Reynolds numbers present in the experiments, it will be interesting to observe the code predictions at the transition time as predicted by theory and observed in experiments. In fact, sudden deviation of simulation from data, after a period of good agreement, may be the best indication that transition has taken place. Continuing improvement in experimental resolution will of course also be helpful. With these tools, we intend to study the dependence of the time to transition on the initial modal spectrum. A key question is how and when will the initial conditions be "forgotten" and the instability proceed into a self-similar regime? Finally, what are the absolute limits of current computer codes and hardware resources in accurately modeling complex highReynolds number flows. These questions must be addressed if a real understanding of the nonlinear hydrodynamics present in core-collapse supernovae is to be attained. 


\section{Chapter 3: The effect of a short wavelength mode on the evolution of a long wavelength perturbation driven by a strong blast wave}

\section{Introduction}

In core-collapse supernovae, the sudden release of an enormous amount of energy near the star's center drives a strong blast wave out through layers of progressively less dense material. ${ }^{1,2}$ The transmission of a blast wave through an interface from a denser to a less dense material constitutes an unstable system. ${ }^{3}$ Preexisting perturbations on the interface grow to larger amplitude after passage of the shock due to two different mechanisms. First, the transmission of a shock through a perturbed interface results in vorticity deposition regardless of whether the traversal is from heavy to light or from light to heavy. The subsequent evolution of the vorticity field leads to perturbation growth, an effect called the Richtmyer-Meshkov (RM) instability. ${ }^{4,5}$ With the passage of a blast wave, the interface begins to decelerate after shock refraction. Since the pressure decreases monotonically with distance behind the shock front, there is an attendant reversal of pressure and density gradients $(\nabla \mathrm{P} \bullet \nabla \rho<0)$ in the heavy to light case. As long as this condition is satisfied at the interface, it is unstable to the Rayleigh-Taylor (RT) instability. ${ }^{6,7}$ Under the influence 
of the RM and RT instabilities, interface perturbations grow into spikes of heavier material "falling into" lighter fluid and bubbles of lighter fluid "rising into" heavier fluid. Shear that develops along the growing spikes drives Kelvin-Helmholtz (KH) growth, which contributes both to the development of characteristic mushroom caps at the spike tips and to the eventual breakup of the interface into a turbulent flow. In addition to core-collapse supernovae, these instabilities will be present during the implosion of inertial confinement fusion (ICF) ignition targets currently being designed for the National Ignition Facility (NIF). For double-shell targets, the resulting mixing of hot fuel with cooler shell material can in turn result in significant reduction or even complete elimination of thermonuclear yield. ${ }^{8}$ Laboratory astrophysics with lasers, which is motivated by both the diagnostic limitations inherent in observational astrophysics and the need to validate the codes used to model astrophysical systems, provides a link between the two classes of applications.

The question of the dependence of RM and RT growth on the initial modal spectrum is at the heart of both astrophysical and ICF applications of compressible mix. This is particularly true for the deep nonlinear and transitional regimes, where linear and weakly nonlinear theories have long-since become inapplicable but the similarity-based scaling arguments commonly applied to the turbulent regime are not yet necessarily valid. The deep nonlinear and transitional regimes must therefore bridge the gap between the earlier phases, where initial conditions have a strong and direct influence on the perturbation growth, and the turbulent regime perhaps characterized by self-similar growth independent of the initial spectrum. Most astrophysical systems of interest are not only turbulent but are very highly turbulent 
from very early times. For example, the Reynolds number in core-collapse supernovae is estimated ${ }^{9}$ to be of order $10^{10}$, many orders of magnitude above the value of $\sim 10^{4}$ suggested by Dimotakis ${ }^{10}$ as sufficient for the mixing transition. According to the analysis by Robey et al of transition in non-stationary flows, transition in supernovae should occur in about 2.8 seconds after the instability initiation. ${ }^{11}$ In simulations by Fryxell et al of SN1987A, perturbations on the $\mathrm{He} / \mathrm{H}$ interface did not grow to significant amplitudes until times of order $10^{3}$ seconds after the explosion while times of interest extend another order of magnitude higher. ${ }^{12}$ If an initial-condition-independent self-similar regime is ever to be attained at a blastwave-driven interface, it must occur virtually instantaneously in a core-collapse supernova if the initial condition are amenable. However, the same cannot be said of the laser-driven high energy density (HED) experiments designed to study such astrophysical events. Present-day experiments can be very nonlinear but are often not turbulent, with Reynolds number much lower than in their astrophysical counterparts (of order $10^{5}$ for those designed to study aspects of supernova hydrodynamics ${ }^{11}$ ) and time scales that are not long compared to predicted transition times. Even if the 1D hydrodynamics is appropriately scaled, the real relevance of "supernova-relevant" experiments is limited by whether or not the level of their dependence on initial conditions is similar to the dependence present in actual supernovae. If the complex nonlinear hydrodynamic mixing in supernovae is in fact initial-condition independent while current experiments are not, then future experiments should move to higher Reynolds numbers and longer time scales. 
Our intent is to study the dependence on initial conditions of blast-wavedriven unstable interface evolution in two phases. In this paper, we consider mostly two-mode interfaces in the regime of recent and current experiments on OMEGA, ${ }^{13}$ and for such cases present a mechanism whereby the unstable evolution of a strongly driven perturbed interface can depend critically on details of the initial mode spectrum. Specifically, we consider how the evolution of a long-wavelength mode is affected by a single short-wavelength component and the dependence of this effect on the relative phases of the two modes. In the second phase, and in a later paper, we will present a study of the dependence on initial conditions for many-mode interfaces under NIF-like drive conditions, in which the degree of phase coherence as well as the spectrum is varied.

We begin with brief descriptions of the experimental setup, relevant previous experiments, the $2 \mathrm{D}$ code CALE,${ }^{14}$ and the simulations. This is followed by a description of the new two-mode interfaces and a discussion of model predictions. The simulation results are presented and shown to exhibit complex behavior that is beyond the reach of existing models. When the drive is sufficiently strong, the nonlinear evolution of the short-wavelength mode can lead to the formation of jets that strongly affect the large-scale structure of the interface. This effect represents a nonlinear coupling between spikes and between spikes and bubbles. We describe the process of jet formation and its impact on the late-time interface evolution, including a significant dependence on the relative phases of the two modes. We compare the simulation results with data from recent two-mode experiments and consider earlier few-mode experiments in the context of these effects. Finally, we conclude with a 
summary of our results and a brief outline of our current work with broadband initial conditions.

\section{Experiments}

The experiment (see schematic in Fig. 1), which is described in greater detail elsewhere, ${ }^{11}$ uses 10 of the OMEGA laser's beams in a $5 \mathrm{~kJ} 1 \mathrm{~ns}$ pulse to drive a Mach 15 blast wave into one end of a cylindrical target. The average laser intensity on the target is typically $6 \times 10^{14} \mathrm{~W} / \mathrm{cm}^{2}$. The target consists of a heavier plastic pusher/ablator section [polyimide $\left(\mathrm{C}_{22} \mathrm{H}_{10} \mathrm{~N}_{2} \mathrm{O}_{4}\right)$ with a density of $1.41 \mathrm{~g} / \mathrm{cc}$ ] and a lighter foam payload section [carbonized resorcinol formaldehyde (CRF) with density $0.05 \mathrm{~g} / \mathrm{cc}]$ in contact with one another along a perturbed interface. In order to reduce lateral expansion, this multicomponent target assembly sits within a Be shock tube. Because the perturbation is machined into the plastic pusher but not into the foam payload, the two materials are actually in contact only at the highest peaks of the perturbation. As a result, there exists a gap between the two materials.

The interface velocity and deceleration (taken from a 1D CALE simulation) are plotted as functions of time in Fig. 2. As the shock front crosses the interface at 1 ns and impulsively accelerates it up to about $70 \mathrm{~km} / \mathrm{s}$, it deposits vorticity, which drives RM growth. The interface then begins to decelerate, and does so for the $40 \mathrm{~ns}$ remainder of the experiment. During the deceleration phase, the interface is RT unstable. Shock-deposited vorticity (RM) dominates the perturbation growth for the 


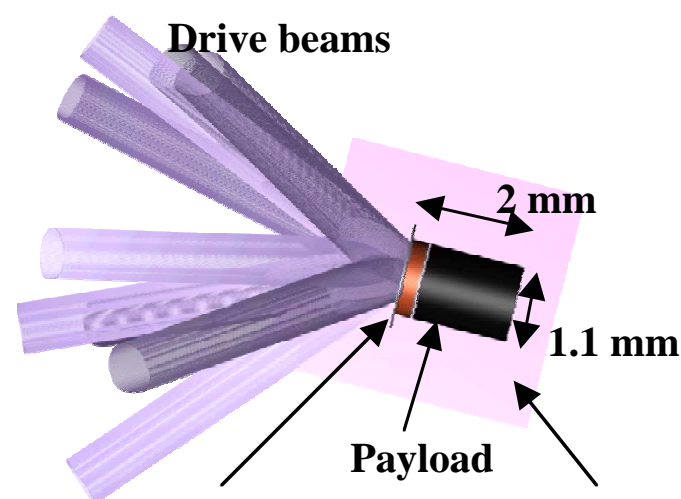

(a)

\section{Ablator/pusher Backlighter}

target

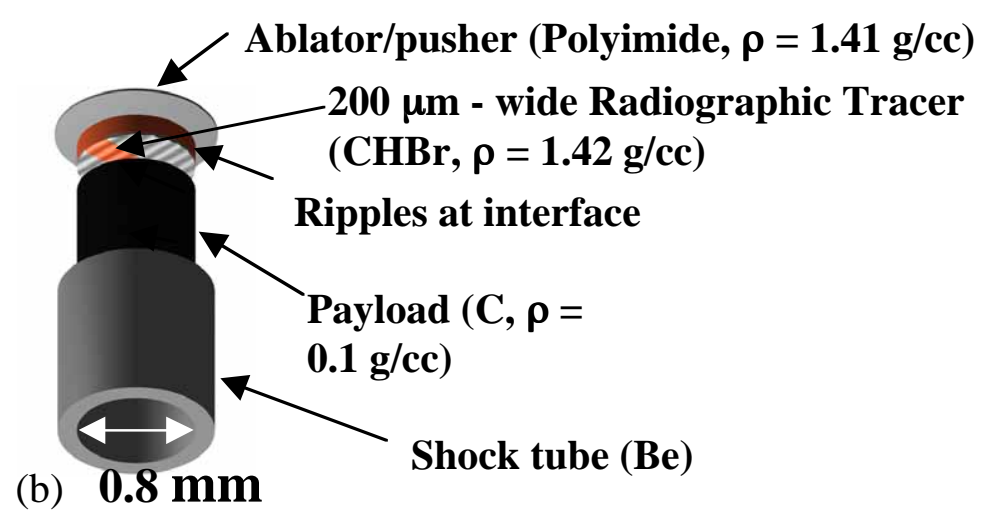

Figure 1: Target schematic showing (a) the overall experiment configuration and (b) an exploded view of the target package. 


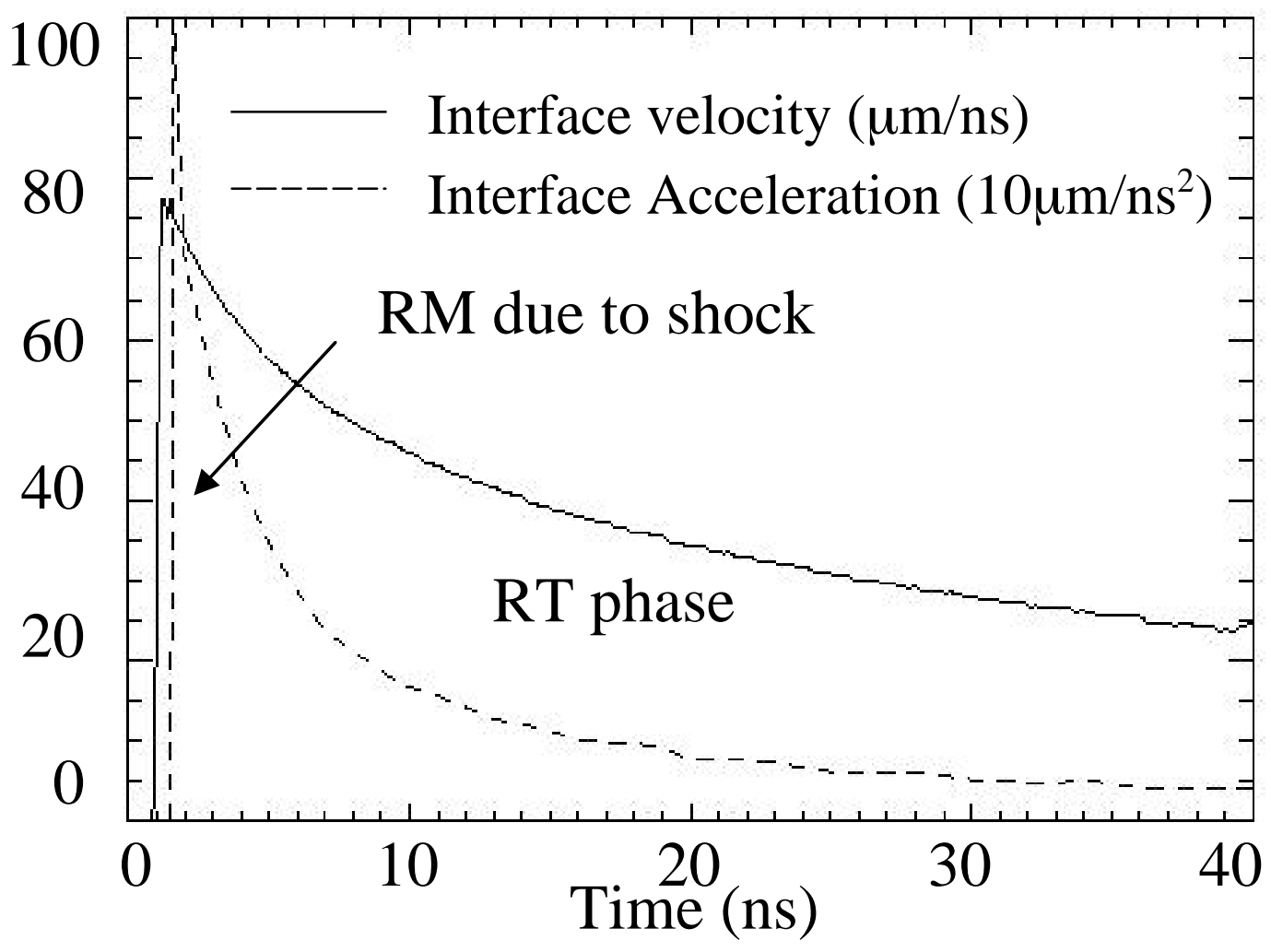

Figure 2: Interface velocity and deceleration. The shock induces RM growth. And the interface is RT unstable during the subsequent deceleration phase. The post-shock Atwood number is nearly constant at $\mathrm{A}^{*}=0.70$.

first couple of nanoseconds, while acceleration-induced (RT) growth dominates at later times. In addition to the RM and RT instabilities, target decompression occurs during the experiment, and is responsible for about $50 \%$ of the total perturbation growth at late times. ${ }^{15}$

The experiments are diagnosed via side-on and face-on x-ray radiography throughout their duration of up to $40 \mathrm{~ns}$. This is done with titanium backlighters that produce $4.7 \mathrm{keV}$ photons for target imaging. A nearly mass-matched radiographic tracer is embedded in the plastic pusher in order to reduce edge effects and concentrate x-ray opacity into a relatively thin slice near the target axis. 


\section{Simulation}

The experiments are simulated using the 2D radiation-hydrodynamics code CALE. CALE is an arbitrary Lagrangian-Eulerian code that uses a second-order (in space and time) finite differencing method to numerically solve the Euler equations. ${ }^{14}$ The relative importance of several numerical and physical factors for achieving good agreement between simulation and experiment were considered in detail in an earlier paper. ${ }^{15}$ In accordance with those results, the simulations in this work are characterized as follows: The computational grid spans the length of the target, and its width is generally determined by the minimum allowable considering symmetry constraints (i.e. one-half wavelength for a single mode simulation). The transverse resolution is 120 points per perturbation wavelength (ppw) of the longest wavelength $(50 \mu \mathrm{m})$ mode. The specified boundary conditions (BC's) are reflecting along the mean flow direction and free along the transverse direction. Simulations are run in Eulerian rather than ALE mode in order to avoid spurious KH activity at the evolving interface. The gap between the plastic and foam sections is included and filled with 1 $\mathrm{mg} / \mathrm{cc}$ air or foam. Electron conduction is included, but the effects of $\mathrm{x}$-ray and electron preheat are not. Tabular equations of state (EOS) are used for all materials, with LEOS or EOP for the plastic and EOP for the foam. Simulations are initialized with a driving slab with uniform temperature, density, and velocity taken from a $2 \mathrm{D}$ LASNEX $^{16}$ calculation. 
Such simulations have been shown to agree well, both in interface structure and spike-bubble averaged perturbation amplitude growth, with experiments involving single-, two-, and eight-mode interfaces. ${ }^{15}$ For the single-mode simulations, separate spike and bubble amplitude histories (as well as their average) were reasonably well predicted by a buoyancy-drag model when target decompression effects are first removed. Aside from the initial modal structure, the earlier experiments differ from those discussed in this work only in the foam density - now 50 rather than $100 \mathrm{mg} / \mathrm{cc}$.

\section{New two-mode interface (short on long) with varying phase}

We now consider the same single-mode perturbation studied previously (with a $50 \mu \mathrm{m}$ wavelength and $2.5 \mu \mathrm{m}$ initial amplitude), and study the effect of a single short wavelength component on its evolution. The scale of the secondary mode (mode 10 ) is one-tenth that of the primary mode (mode 1 ), or $5 \mu \mathrm{m}$ in wavelength and 0.25 $\mu \mathrm{m}$ in initial amplitude (see Fig. 3). With $120 \mathrm{ppw}$ in mode 1, mode 10 is resolved in the simulations to $12 \mathrm{ppw}$. According to Ofer et al, ${ }^{17}$ the finite numerical resolution of an RT-unstable mode (for incompressible flow under constant acceleration) results in a growth rate reduction below the theoretical value that is given by

$$
\gamma_{\text {eff }}=\gamma_{\text {theoretical }}(1-2 / \mathrm{ppw})
$$

For mode 10 , Eq. (1) predicts a $17 \%$ reduction in growth rate relative to the fully resolved value. 


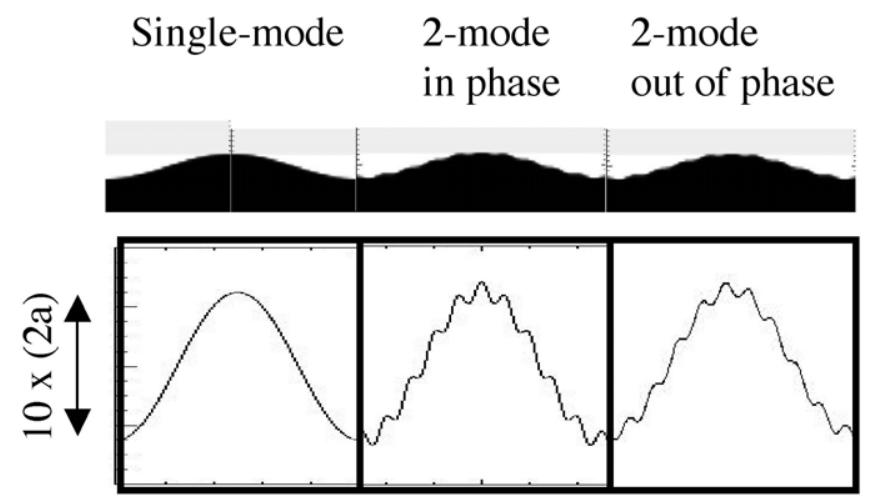

Mode 1: $\lambda=50 \mu \mathrm{m}, \mathrm{a}=2.5 \mu \mathrm{m}(\mathrm{ka}=0.3), 120 \mathrm{ppw}$ Mode 10: $\lambda=5 \mu \mathrm{m}, \mathrm{a}=0.25 \mu \mathrm{m}(\mathrm{ka}=0.3), 12 \mathrm{ppw}$

Figure 3: Single and two-mode initial interfaces. The short wavelength mode (mode 10 ) is either in phase or $\pi / 2$ out of phase with the long wavelength mode (mode 1 ).

Ofer et al, in their consideration of the effect of a secondary short wavelength on a primary long-wavelength mode, found that the main effect of the short wavelength component was to introduce an effective density gradient that acted to stabilize the growth of the primary mode. ${ }^{18}$ They used a 2D ALE code in their study and, like us, considered a moderate Atwood number $(\mathrm{A}=0.5$ compared to our postshock $\left.A^{*}=0.7\right)$. Their calculations were slightly better resolved than ours, with $17-18$ points per wavelength ( $\mathrm{ppw}$ ) in the shortest wavelength mode (compared to our value of $12 \mathrm{ppw}$ in the shortest wavelength mode). There are, however, several significant differences between their study and ours. Theirs was a pure RT system (no RM) with constant acceleration, while our blast-wave-driven interface is both RM and RT unstable with decaying acceleration. Our instability Mach number is higher, with $\mathrm{M}=$ $0.15-0.20$ in foam and $0.25-0.40$ in plastic, than their $\mathrm{M}<0.1$. It is also significant that their long wavelength mode remains linear throughout, while both of our modes 
enter the nonlinear regime early on. Finally, their short wavelength component is in phase (or 180 degrees out of phase, depending on one's choice of the positive direction). In order to investigate the phase dependence of the instability in the deep nonlinear regime, the relative phase of mode 10 with respect to mode 1 is in our study either $0, \pi / 4$, or $\pi / 2$.

With a phase difference of 0 or $\pi$, the initial symmetry allows us to limit the computational domain to one half of the mode 1 wavelength. Since CALE does not allow for periodic BC's, a reasonable treatment of the out-of-phase case requires that we include multiple wavelengths. This requirement must be balanced with the need to run with reasonably high resolution and in a reasonable amount of CPU-time. In light of these considerations, we include four mode 1 wavelengths in the out-of-phase case. The two modes are then technically modes 4 and 40, but because the ratio of their wavelengths is an integer, no modes lower than 4 can be generated via mode coupling. Consequently, the system is equivalent to the mode $1 /$ mode 10 system in the region acoustically isolated from the boundaries (while such a region exists). Late time density plots from the out of phase simulation show some competition between mode 4 bubbles, but not merger and the associated generation of larger, faster growing structures.

\section{$\underline{\text { V. Model predictions }}$}


In considering model predictions, we divide the perturbation evolution into three regimes: linear, early nonlinear, and nonlinear. Simple theory predicts that, neglecting certain potentially stabilizing factors such as surface tension, ${ }^{19}$ each RTunstable mode grows exponentially in the linear regime $(\mathrm{a} / \lambda<0.1)$ with a growth rate given by ${ }^{20}$

$$
\gamma=\sqrt{\frac{k g A}{1+k L}}
$$

where $\mathrm{k}$ is the perturbation wavenumber $2 \pi / \lambda, \mathrm{g}$ is the (constant) acceleration, $\mathrm{A}$ is the Atwood number, and $\mathrm{L}$ is the density gradient scale length at the interface. Thus the RT exponential growth rate (1/a)da/dt for mode 10 is greater than for mode 1 by a factor of $\sqrt{ } 10$ (i.e. $\gamma_{\text {mode } 10}=\sqrt{ } 10 \gamma_{\text {mode } 1}$ ). The amplitude growth rate da/dt of mode 10 is smaller than for mode 1 by the same factor $\left(\mathrm{da} / \mathrm{dt}_{\text {mode } 10}=\mathrm{da} / \mathrm{dt}_{\text {mode } 1} / \sqrt{ } 10\right)$.

According to Richtmyer's impulsive RM model ${ }^{4}$ adapted by Meyer and Blewett for the heavy to light case,${ }^{21}$ each RM-unstable mode amplitude grows linearly in time during the linear phase at the Meyer-Blewett velocity, given by

$$
\mathrm{v}=\mathrm{k} \mathrm{a} \mathrm{a}_{0} \mathrm{u}_{\mathrm{i}}
$$

Here $a_{0}$ is the average of the pre- and post-shock perturbation amplitudes, $A^{*}$ is the post-shock Atwood number, and $\mathrm{u}_{\mathrm{i}}$ is the velocity increase of the interface upon shock transmission. Because both the amplitude and wavelength of the short-wavelength mode are scaled in the same way relative to long-wavelength mode, the MeyerBlewett velocity is the same for modes 1 and 10 .

According to linear modal analysis where modes grow independently without interacting, mode 10 can have no effect on mode 1 regardless of the relative phase between the two modes. Ofer et al found that the short wavelength mode does 
introduce an effective density gradient at the interface that will somewhat stabilize the primary mode (via L in Eq. 2). ${ }^{18}$ According to their analysis, the mode 1 linear RT growth rate is reduced by $10 \%$ when $(\mathrm{a} / \lambda)_{10}$ reaches about 0.3 , or about the time mode 10 reaches its saturation velocity.

Because the targets under consideration are driven very strongly and the initial amplitudes are somewhat large (pre-shock $\mathrm{a} / \lambda=0.05$ and post-shock $\mathrm{a}^{*} / \lambda \approx 0.02$ ), the linear approximation is valid for a very short period of time. Considering RT only, modes 1 and 10 reach the nonlinear threshold value of $\mathrm{a} / \lambda=0.1$ in about $2 \tau^{\mathrm{RT}} \approx 2.0$ and 2/3 ns, respectively. For RM only, the linear approximation breaks down in about $\tau_{\mathrm{nl}}^{\mathrm{RM}} \approx 0.5$ and $0.05 \mathrm{~ns}$ for mode 1 and mode 10 , respectively. Thus RM growth, which dominates for the first couple of ns, provides the stronger limit on the linear regime. Mode 1 becomes nonlinear within 1 ns (of a 40 ns experiment) and mode 10 is nonlinear virtually instantaneously.

At later times, initially sinusoidal perturbations grow into characteristic spikes and bubbles. In the early nonlinear phase, which is also very short, mode coupling is present but weak, and Haan's spectral model ${ }^{22}$ is valid. Harmonic generation (of modes 2 and 20) introduces spike-bubble asymmetry, with spikes growing faster than bubbles. In addition, modes 1 and 10 couple to generate modes 9 and 11 .

The saturation velocity of the primary mode can in principle be reduced by the presence of a second mode where the two interfere constructively. ${ }^{23}$ Mode 10 interferes constructively with mode 1 at each mode 1 bubble tip when the two are in phase. However, because of their large separation in wave-number space, both modes should saturate at the single mode terminal velocity. Modes subsequently generated 
by mode coupling should also not reduce the mode 1 saturation time for the same reason and also because of how quickly mode 1 becomes nonlinear.

The early nonlinear phase ends when third order terms (and soon thereafter terms of all order) in the perturbation expansion become significant and the bubbles approach terminal velocity. For constant acceleration $\mathrm{RT}$ with $\mathrm{A}=1$, the bubbles rise with constant (terminal) velocity while spikes fall with constant acceleration in the nonlinear regime. ${ }^{24,25}$ When $\mathrm{A}<1$, the spike also reaches terminal velocity. ${ }^{25}$

Mode coupling between modes 1 and 10 is fairly weak ( $3^{\text {th }}$ order). In addition, Ofer et $\mathrm{ll}^{17}$ found that once a mode has reached its saturation amplitude, it no longer contributes to the growth of longer wavelength modes. Since mode 10 becomes nonlinear in well under a nanosecond, it does not have time to significantly affect the interface evolution via mode coupling. During the nonlinear phase, bubbles (and spikes for $A<1$ ) grow at terminal velocity $\propto \sqrt{\lambda}$, where $\lambda$ is the object's transverse size. ${ }^{24}$ Since larger structures grow faster, an inverse cascade driven by bubble competition and merger sets in and washes small-scale bubbles downstream. ${ }^{26,27}$

The coupling strength in modal models ${ }^{22,17}$ depends on the relative phases of interacting modes according to $\cos \left(\varphi_{\mathrm{j}} \pm \varphi_{\mathrm{k}}-\varphi_{\mathrm{i}}\right)$ for mode $\mathrm{i}$ driven by the interaction of modes $\mathrm{j}$ and $\mathrm{k}$. The plus sign is mode $\mathrm{i}$ generated by shorter wavelength modes (harmonic generation) while the minus sign is for mode i generated by the interaction of longer wavelength modes. Thus the coupling increases with increasing phase coherence and vanishes for modes that are out of phase.

The late-time scaling is determined by the nature of the time-dependent acceleration. If $g(t)$ falls off slower than $t^{-2}$, then the instability growth is $\mathrm{RT}$ rather 
than RM like. ${ }^{28}$ For an n-dimensional blast wave in the self-similar regime, dimensional analysis requires that the shock-front deceleration scales like $\mathrm{t}^{-2(\mathrm{n}+1) /(\mathrm{n}+2)}$. Away from the shock front, the resulting deceleration of the driven interface scales like $\mathrm{t}^{-2\{1-1 /[\gamma(\mathrm{n}+2)]\}}$, where $\gamma$ is the adiabatic index. In the infinitely compressible case $(\gamma=$ 1), the exponent is equal to $-4 / 3$ when $n=1$ and is greater than -2 for all positive $n$. For any dimension $n$, the exponent approaches -2 from above as the adiabatic index becomes large. Consequently, neglecting the true RM component associated with the passage of the shock front, perturbation growth at a blast-wave-driven interface is always RT-like. The bubble distribution approaches a scale-invariant attractor and then the growth of the bubble and spike fronts scales like ${ }^{29,30} h_{s, b} \sim \alpha_{s, b} A\left[\int d t \sqrt{g}\right]^{2}$, or $\mathrm{h}_{\mathrm{s}, \mathrm{b}} \sim \alpha_{\mathrm{s}, \mathrm{b}} \mathrm{At}^{2 /(3 \gamma)}$ for a 1D blast wave (neglecting material decompression). This follows from the assumption that the height of the front is proportional to the dominant wavelength or transverse bubble size and gives the well-known quadratic dependence for constant acceleration. ${ }^{31}$ In experiments and simulations, however, the inverse cascade to successively larger structures is limited by the size of the physical or computational box. In our simulations, which are intended to study the effect of a high 1-mode on a dominant low l-mode, the box size is not large compared to the wavelength of the lowest 1 mode with significant initial amplitude (mode 1 for the single mode and in-phase cases and mode 4 for the out-of-phase case). Once mode 1 becomes the fastest growing mode (which is true very early on due to its large initial amplitude) a scale-invariant bubble distribution cannot be attained. If we therefore assume that the dominant wavelength remains constant and the acceleration falls off like $\mathrm{t}^{-2[1-1 /(3 \gamma)]}$, then we find that $\mathrm{h} \sim \mathrm{t}^{1 /(3 \gamma)}$. This asymptotic behavior is captured by 
buoyancy-drag models such as that of Oron et al, where the spike (s) and bubble (b) evolution are governed by the equations ${ }^{32}$

$$
\left(\rho_{b, s}+2 \rho_{s, b}\right) \frac{d u_{b, s}(t)}{d t}=\left(\rho_{s, b}-2 \rho_{b, s}\right) g(t)-\frac{6 \pi}{\lambda} \rho_{s, b} u_{b, s}^{2} .
$$

In order to make some prediction of the late-time dependence on the initial phases, we have applied the modal model of Ofer et a ${ }^{17}$ with some modifications. In the early nonlinear stage, mode growth and coupling are determined by Haan's weakly nonlinear model. ${ }^{22}$ Unlike Haan's model, modes generated by mode coupling can couple with each other and with preexisting modes. This amounts to an approximate inclusion of higher order terms, thereby extending the model's range of validity. Modes saturate according to the Haan criterion ${ }^{23}$ and subsequently do not contribute to the growth of lower l-modes. Saturated low l-modes can, however, modify the phases of higher 1-modes through harmonic generation as long as the velocity of the driven mode does not exceed its saturation value. In our application of the model, the time dependence of the acceleration is included. In addition, our treatment of phases is more general than in the original implementation, which effectively allowed for cosine modes with phases of only 0 and $\pi$. In our case, the coupling term includes the $\cos \left(\varphi_{\mathrm{j}} \pm \varphi_{\mathrm{k}}-\varphi_{\mathrm{i}}\right)$ term. When the interaction of two modes generates a new mode, the phase of the new mode is determined by the resonance condition $\varphi_{\mathrm{i}}=\varphi_{\mathrm{j}} \pm \varphi_{\mathrm{k}}$. When an existing mode $\mathrm{i}$ is driven by two modes $\mathrm{j}$ and $\mathrm{k}$, producing a velocity increment $\mathrm{dv}_{\mathrm{i}}$, the phase of the driven mode is shifted according to

$$
\varphi_{i}^{n}=\tan ^{-1}\left[\frac{a_{i}^{n-1} \sin \left(\varphi_{i}^{n-1}\right)+d v_{i} d t \sin \left(\varphi_{j} \pm \varphi_{k}\right)}{a_{i}^{n-1} \cos \left(\varphi_{i}^{n-1}\right)+d v_{i} d t \cos \left(\varphi_{j} \pm \varphi_{k}\right)}\right]
$$


where $\mathrm{n}$ is the time step and $\mathrm{a}_{\mathrm{i}}$ is the amplitude of mode $\mathrm{i}$. This follows from the requirement that $a_{i} \cos \left(k_{i} x+\varphi_{i}^{n}\right)=a_{i}^{n-1} \cos \left(k_{i} x+\varphi_{i}^{n-1}\right)+d v_{i} d t \cos \left(\left(k_{j} \pm k_{k}\right) x+\varphi_{j} \pm \varphi_{k}\right)$ since, by definition, $k_{i}=k_{j} \pm k_{k}$.

The result is shown in Figure 4. For the bubble amplitude, the model predicts essentially no late time dependence of the large-scale structure on the initial presence of mode 10 , let alone on its phase relative to that of mode 1 . There is a strong effect predicted for the spike amplitude. However, since the spike evolution will clearly be strongly affected by $\mathrm{KH}$ rollup long before it reaches the shape predicted by the modal model, this prediction should not be taken too seriously. In fact, if we define the spike position as the point at which the spike width falls below $2 \mu \mathrm{m}$ (approximately equal to the width of four computational cells and the minimum spike width observed in the simulations), then the late-time effect of mode 10 on the spike amplitude virtually disappears.

In conclusion, the models considered predict that the effect of mode 10 on mode 1 via mode coupling will be weak at all times. Consequently, the main effect of mode 10 on the evolution of mode 1 should be a reduction of the growth of mode 1 due the effective density gradient provided by mode 10 .

There are several other factors not discussed here that could in principle play a role in the interface evolution. Of these, we are particularly interested in the late-time effect of the RM component, material decompression (particularly the resulting vortex dynamics in experiments that are best only quasi-2D), the relative contributions of electron conduction, viscosity, and finite resolution on stabilization of high-1 modes, reduction of Atwood number in the mixing layer due to the $\mathrm{KH}$ 


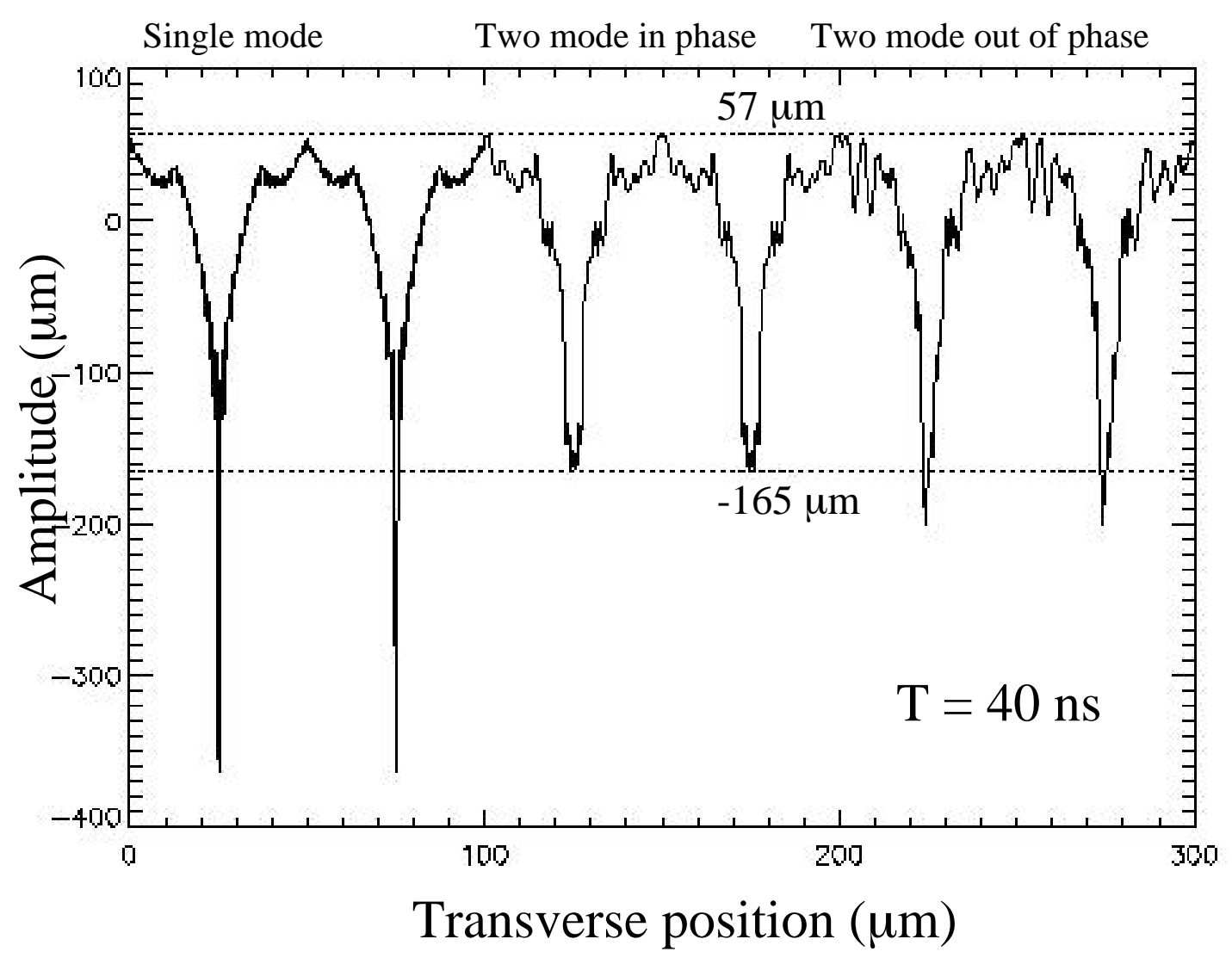

Figure 4: Modal model prediction of interface at $40 \mathrm{~ns}$. The upper dotted line denotes the bubble position $(57 \mu \mathrm{m})$, which is approximately equal for all three cases. The lower doted line (at $-165 \mu \mathrm{m}$ ) shows the approximate position at which the predicted spike widths fall below the minimum value observed in the simulations (about $2 \mu \mathrm{m}$ ). Like the predicted bubble amplitude, the spike amplitude defined in this way is virtually the same for all three cases. The Kelvin Helmholtz instability prevents the spikes from attaining shapes predicted by the model. The model also does not include material decompression and RM contribution.

instability, and reduction in Atwood number at the spike and bubble tips due to the large-scale density gradient present behind the shock front. Some of these issues will be treated in a later paper in the context of broadband initial conditions. 


\section{Simulation results}

The averaged amplitude histories for the different phase realizations (including mode 10 by itself) are shown in Fig. 5. Also included is the mode 1 prediction of Oron et al's buoyancy-drag model (given in Eq. 5).$^{32}$ In order to isolate the instability effects and facilitate comparison with the model, the effect of target decompression has been removed from all amplitude plots. ${ }^{15}$ As expected, the linear phase lasts no more than about $1 \mathrm{~ns}$. Before $10 \mathrm{~ns}$, the overall growth is not strongly affected by the presence of the short wavelength mode. Consequently, the singlemode buoyancy-drag model provides a good description of the growth in all three cases. While the acceleration is large, Mode 10 remains sufficiently small that the introduction of its amplitude into the buoyancy-drag model as a stabilizing density gradient results in virtually no change in the predicted mode 1 growth.

At intermediate times (between 10 and $20 \mathrm{~ns}$ ), the multimode perturbation amplitude histories reveal abrupt changes in the growth rates. After first falling below the single mode case, the growth of the in-phase interface suddenly increases, so that its final amplitude $(140 \mu \mathrm{m})$ is somewhat higher than in the single mode case (133 $\mu \mathrm{m})$. The growth rate in the out-of-phase case also falls below the single mode case at about $10 \mathrm{~ns}$. At about the time that the in-phase growth rate increases, however, the out-of-phase growth rate falls still further, so the averaged amplitude reaches only 80 $\mu \mathrm{m}$ at $40 \mathrm{~ns}$. The result is that, after $20 \mathrm{~ns}$, the phase-correlated noise leads to some 


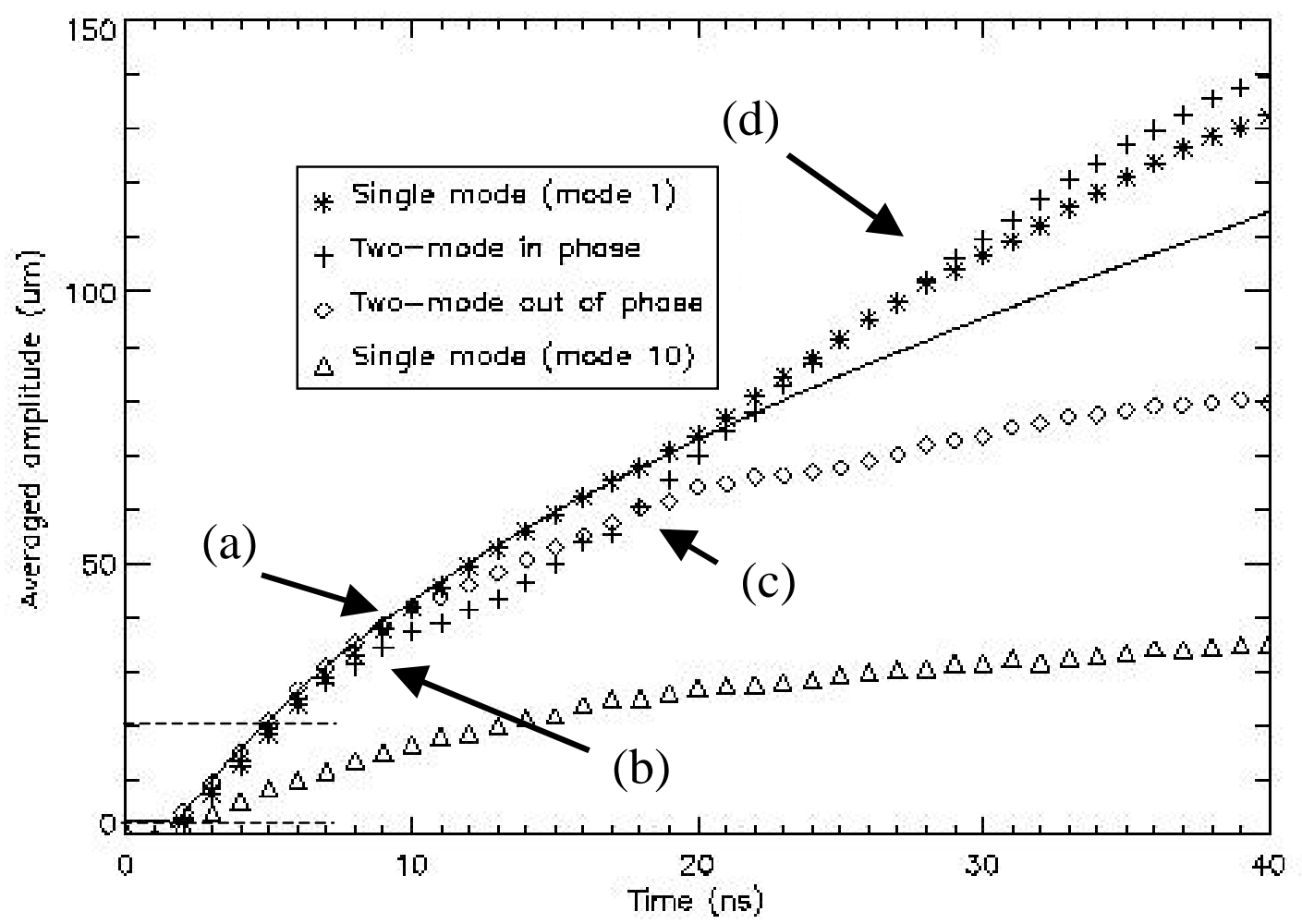

Figure 5: Spike-bubble averaged amplitudes corrected for decompression. (a) The bubble-merger-driven inverse cascade is complete by about $10 \mathrm{~ns}$. (b) Early on, the growth is not strongly affected by short- $\lambda$ "noise". (c) For the two-mode cases, sudden changes in growth rate occur at intermediate times. (d) After $20 \mathrm{~ns}$, the phasecorrelated (decorrelated) noise leads to growth enhancement (suppression) relative to single mode. The dashed lines show the single-mode saturation values $(\mathrm{a} / \lambda=0.4)$ for modes 1 (upper) and 10 (lower).

growth enhancement (by about 5\%) relative to the single mode while the phase decorrelated noise leads to tremendous growth suppression (by about 40\%).

Additional detail can be obtained from plots of separate spike and bubble amplitude histories, which are shown in Fig. 6. Without the short wavelength component, the mode 1 amplitudes are as expected for an interface driven by a decaying acceleration. The spike and bubble growth rates fall off with the acceleration, and the amplitudes begin to saturate as the drive strength approaches zero, with the spike amplitude significantly larger than that of the bubble. The spike- 

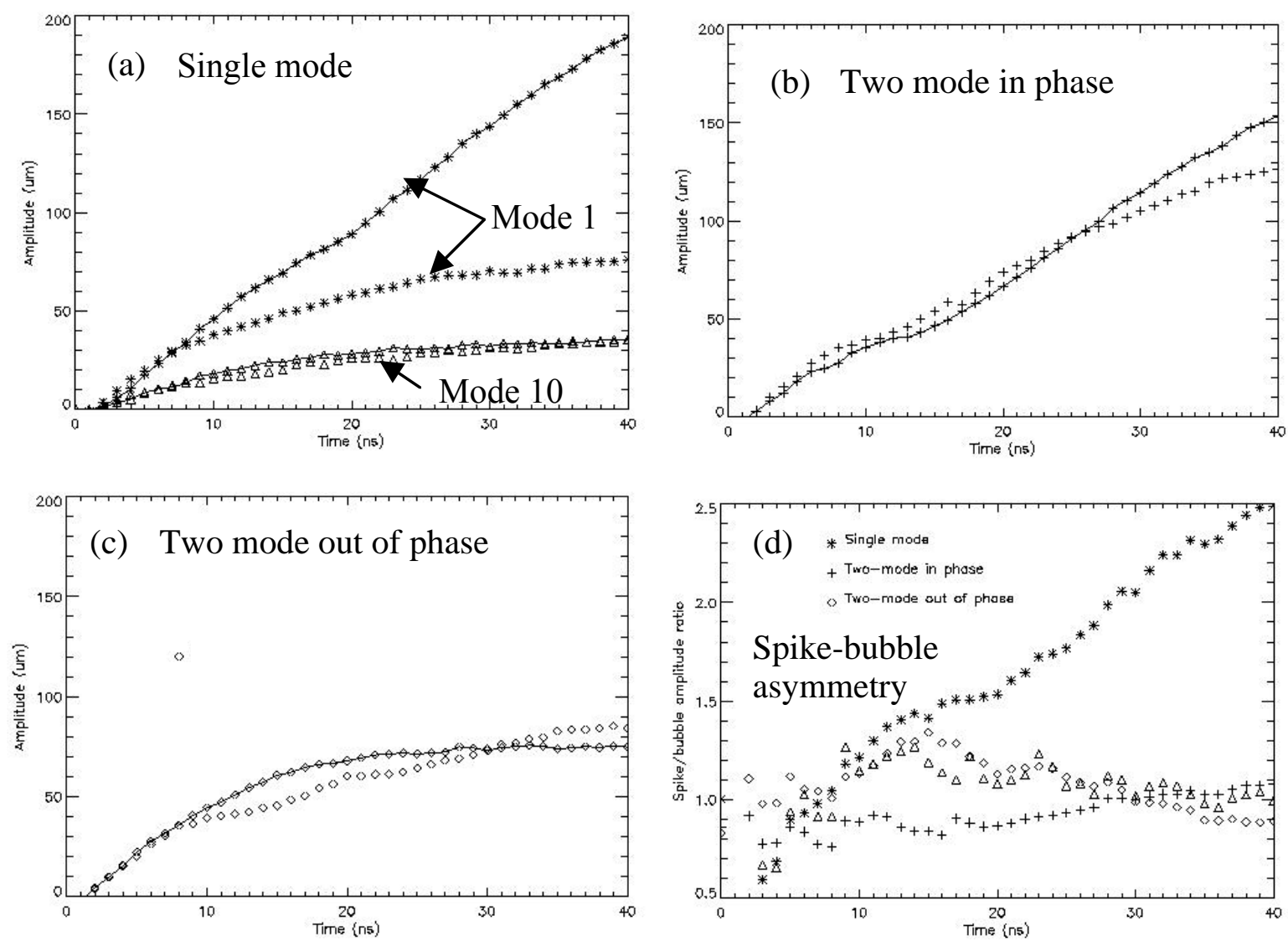

Figure 6: Separate spike and bubble amplitudes corrected for decompression. In each case, the line denotes the spike amplitude. (a) The mode 1 single mode growth is as expected, with the spike amplitude significantly larger than that of the bubble at late times. The spike and bubble growth is nearly symmetric in the mode 10 single mode case because of the effective Atwood number reduction due to the density gradient at the interface. (b) In the two mode in-phase case, there is some spike growth reduction relative to the single mode case, and tremendous bubble growth enhancement. (c) In the two mode out of phase case, there is slight bubble growth enhancement and tremendous spike growth reduction. (d) A plot of the spike-bubble asymmetry (the ratio of spike to bubble amplitudes) shows nearly symmetric growth in two mode cases.

bubble symmetry apparent in the mode 10 simulation likely results from an effective low Atwood number due to the finite density gradient at the interface, which is significant compared to the early-time amplitude of mode 10 but not mode 1 . The two-mode-in-phase case begins similarly, but the bubble growth rate suddenly increases at about $11 \mathrm{~ns}$. Late in time, the spike amplitude is somewhat reduced 
relative to the single-mode case (by less than $2 \%$ ), while the bubble shows tremendous growth enhancement (about 65\%). In fact the bubble amplitude is greater than the spike amplitude until about 25 ns. The out-of-phase case strongly differs from both the in-phase and the single-mode cases. The bubble growth is again enhanced, though only slightly (by about 1\%), but there is tremendous reduction of the spike growth (by about $60 \%$ ). In contrast with the single-mode case, the spike and bubble growth are nearly symmetric in both of the two-mode cases.

In summary, mode 10 has little effect on mode 1 during the linear and early nonlinear phases of the instability evolution, but has a strong effect during the deep nonlinear phase when the driving acceleration has decayed to below $25 \%$ of its peak value. The effect can lead to either reduction or enhancement of the overall amplitude growth depending on the phase of mode 10 relative to mode 1 . In both cases, the short wavelength noise has a symmetrizing effect on the spike-bubble growth. The phasecorrelated noise causes some growth enhancement relative to the single-mode case while the phase-decorrelated noise results in tremendous growth reduction.

Furthermore, this effect does not appear to result from mode 10 providing an effective density gradient at the mode 1 interface.

The operative mechanism can be understood by observing the interface as it evolves (see Figs. 7-10). At 2 ns [1 ns after shock refraction - see Fig 7(a)], both modes are apparent in the two-mode cases, and the effect of mode 10 on mode 1 is clearly small. At this point mode 1 is just entering the early nonlinear phase $(\mathrm{a} / \lambda=$ 0.1 ) while mode 10 has already attained $\mathrm{a} / \lambda=0.4$. In the single-mode case, $\mathrm{KH}$ rollup at the spikes is visible within $3 \mathrm{~ns}$ of the interaction time. In the two-mode cases, the 

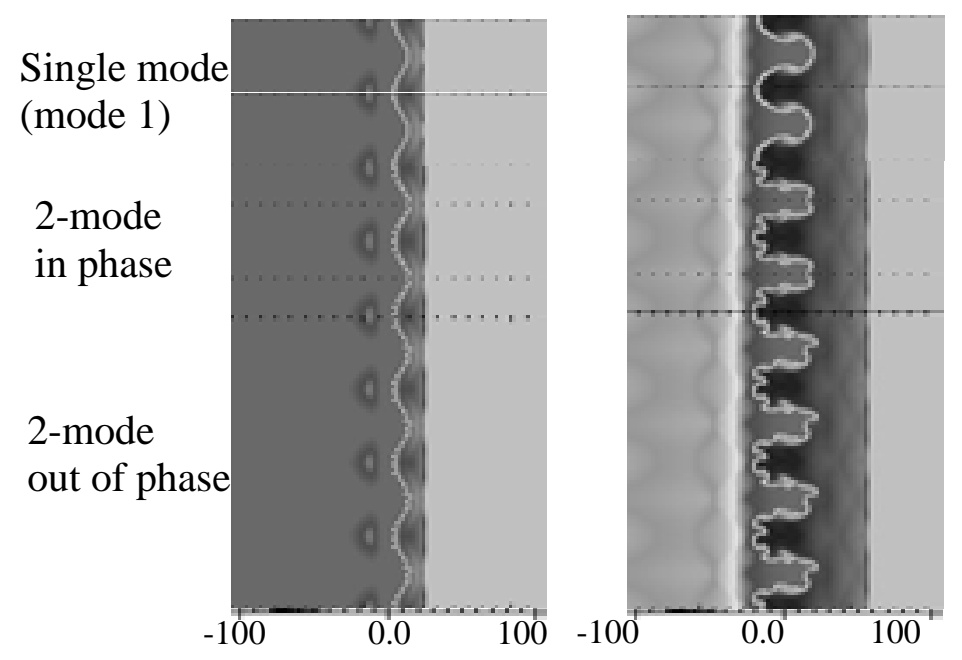

(a) $2 \mathrm{~ns}$

(b) $4 \mathrm{~ns}$
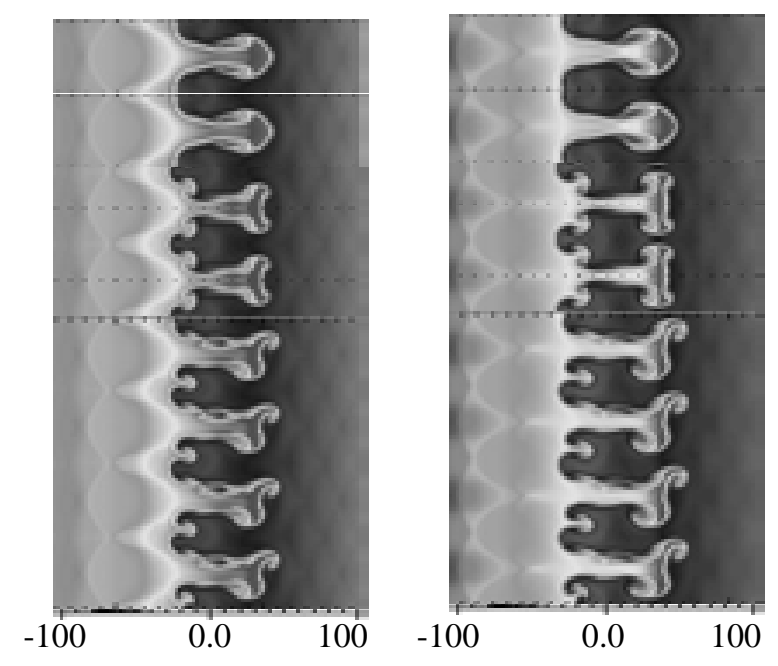

(c) $6 \mathrm{~ns}$

(d) $7 \mathrm{~ns}$

Figure 7: Density plots at (a) 2 ns, (b) 4 ns, (c) 6 ns, and (d) 7 ns. At $M>0.1$, the drag force affects not only the saturation velocity, but also the shape of the spike and bubble. 


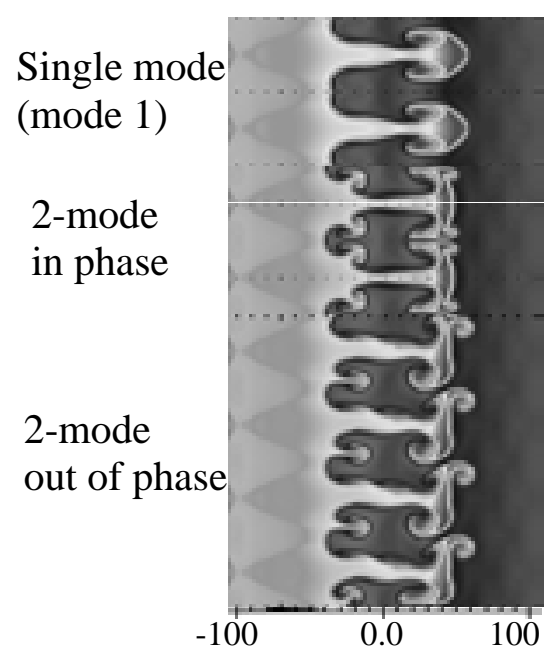

(a) $8 \mathrm{~ns}$

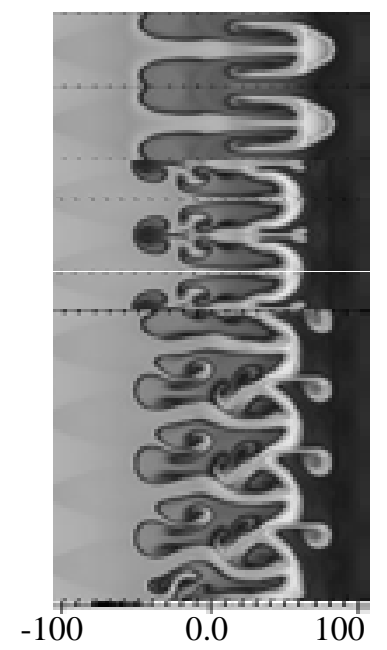

(c) $11 \mathrm{~ns}$

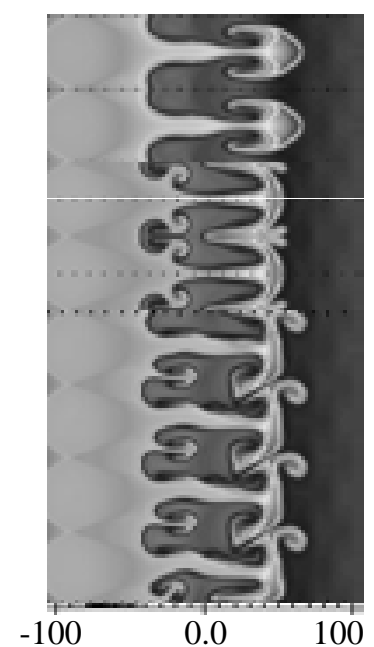

(b) $9 \mathrm{~ns}$

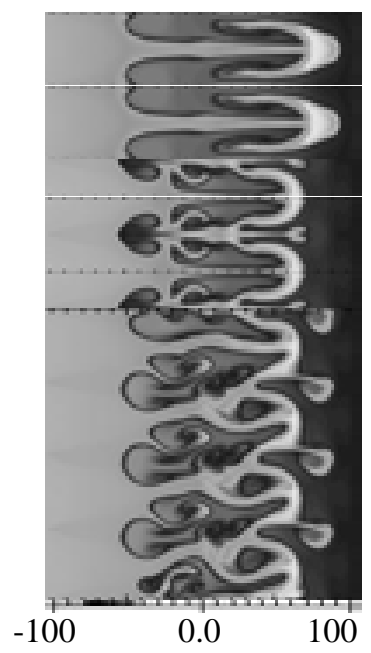

(d) $12 \mathrm{~ns}$

Figure 8: Density plots at (a) 8 ns, (b) 9 ns, (c) $11 \mathrm{ns,} \mathrm{and} \mathrm{(d)} 12$ ns. Deflection of spikes results in colliding spikes, especially for interfaces consisting of periodic arrays of spikes (phase coherence). Fast colliding spikes drive premature bubblemerger and produce upwards and downwards-directed jets, with most of the energy directed down for collision perpendicular to the zero-order flow velocity direction. Downwards-directed jets strike the inner surface of bubble tips, thereby depositing energy that accelerates bubble growth. 

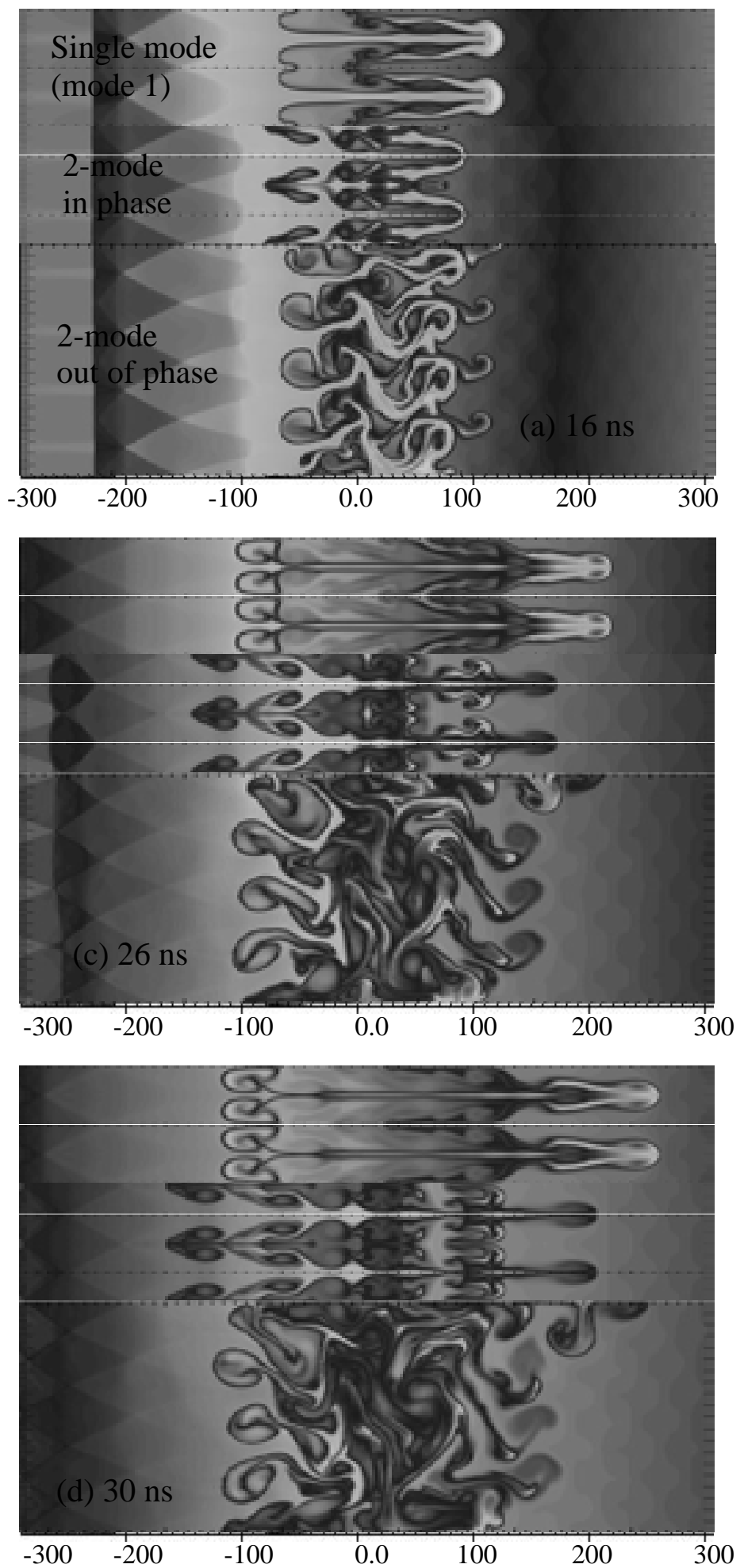

Figure 9: Density plots at (a) $16 \mathrm{~ns}$, (b) $26 \mathrm{~ns}$, and (c) $30 \mathrm{~ns}$. Coupling of the spike interaction process with the $\mathrm{KH}$ instability results in additional coupling between and generation of scales and greatly enhanced mixing in the layer. This is a complicated nonlin. transfer of energy from spikes to bubbles and from transverse to parallel KE. 

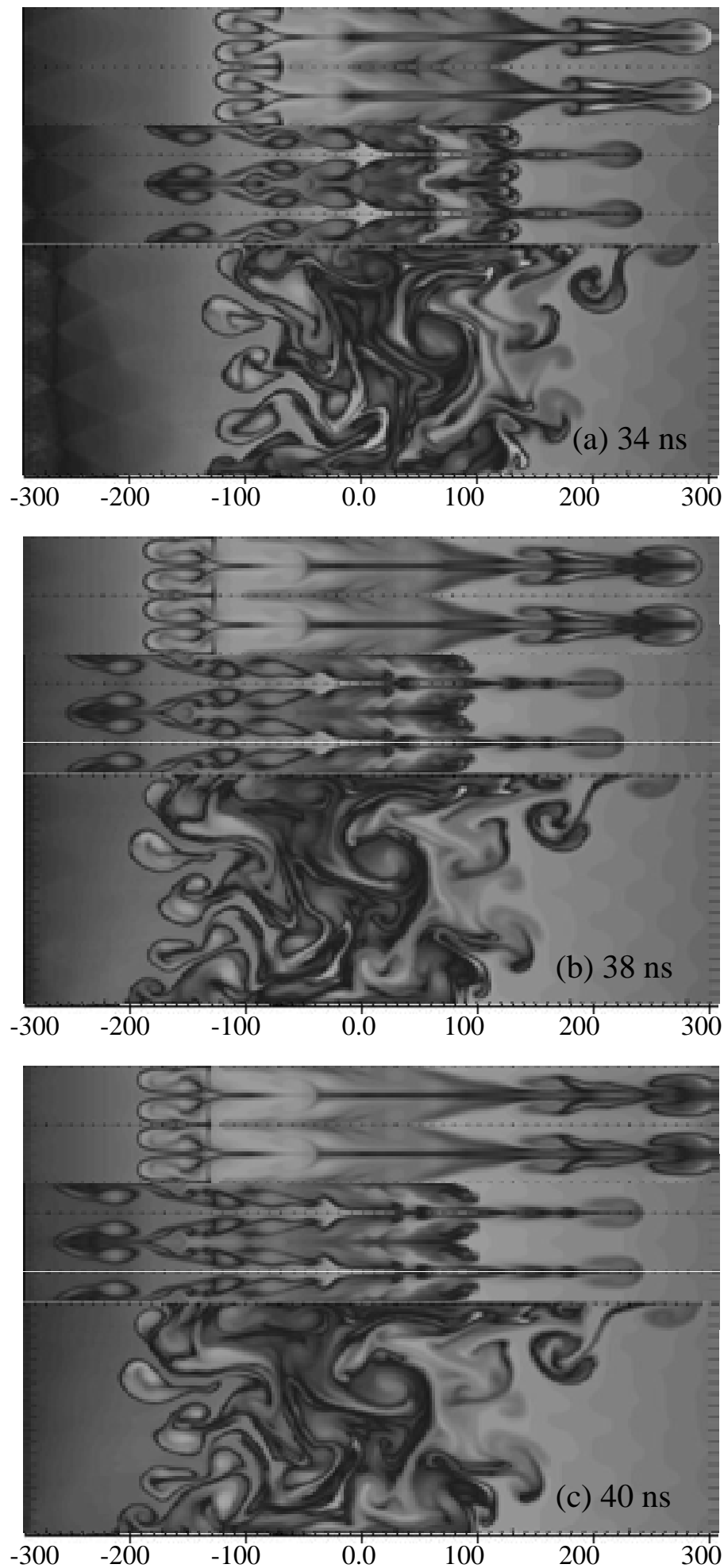

Figure 10: Density plots at (a) $34 \mathrm{~ns}$, (b) $38 \mathrm{~ns}$, and (c) $40 \mathrm{~ns}$. The result is that largescale features present during late nonlinear instability evolution are strongly affected by small-scale details of the initial conditions. In this case, phase coherence results in increased bubble growth while phase decoherence gives spike growth suppression. 
bubble merger process proceeds rapidly. The ten small-scale bubbles per mode 1 wavelength present at 2 ns merge into five by 4 ns, and then into two or three (depending on the phase) by $6 \mathrm{~ns}$. After one more merger, the process is complete by 10 ns. By 5 ns [see Figs. 7(b)-7(c)] the shape of the primary spikes has been significantly altered by the presence of mode 10 . The remaining secondary spikes (bubble merger is already underway) near the tips of the primary spikes have acquired a transverse growth component that is particularly pronounced in the in-phase case [see Fig. 7(d)]. In the in-phase case, pairs of transversely growing secondary spikes collide with one another at about 8 ns [Fig. 8(a)], driving premature bubble merger and producing upstream and downstream-directed jets. Since the collision direction is nearly perpendicular to the main flow direction, most of the collision energy is directed downstream. In the out-of-phase case, only a grazing collision occurs because every other of the secondary spikes (at the primary spike tips) still has a significant upstream velocity component. As a result, half of these secondary spikes are directed downward and eventually strike the primary spike stalks at about $11 \mathrm{~ns}$ [Fig. 8(c)]. This in turn causes a sudden reduction in the spike amplitude growth rate and leads to the large reduction in spike growth relative to the single-mode case observed at late times. Also at about $11 \mathrm{~ns}$, the downstream-directed jets produced in the in-phase case strike the inner surface of the primary bubble tips, thereby depositing energy that suddenly accelerates the bubble growth. At later times, $\mathrm{KH}$ activity near the primary spike tips effectively regenerates the smaller scales lost due to bubble merger. The process of secondary spike collision and jet formation can then continue, particularly in the in-phase case. This occurs, for example, between 26 and 
30 ns (see Fig. 9). Each new jet sends more spike material downstream into the primary bubble region, so that the coupling between $\mathrm{KH}$ and secondary spike interaction results in greatly enhanced mixing in the layer in addition to additional coupling between and generation of scales.

In the out-of-phase case, a large-scale vortex begins to form across several mode 1 wavelengths between 26 and 30 ns. This signifies that the edges of the computational domain have begun to influence the interface evolution all along the transverse direction. By this time, however, the spike amplitude in the out-of-phase case has already been greatly reduced relative to the other cases and has nearly saturated.

At very late times, there are large differences in the interface structure of all three similations (see Fig. 10). Thus the large-scale features present during the late nonlinear instability evolution are strongly affected by the details of the initial conditions. Not only the presence of the short-wavelength mode, but also its phase, has a dramatic impact on the final state.

\section{Comparison with experimental data}

Two sets of two-mode short on long experiments have been performed at Omega to investigate the effect of a short wavelength secondary mode on the evolution of a long-wavelength primary mode. The wavelengths and initial amplitudes of the two modes are the same as in the simulations. The first series of 
four targets was shot in April 2003. In an effort to obtain a true side-by-side comparison unaffected by shot-to-shot variations in drive energy, the interface in each of these targets was divided in two regions - single-mode on one side and two-mode on the other. Unfortunately, all information on which side of each target was single mode and which was two-mode was lost. Figure 11(a) shows a radiograph from one of these shots (half single mode/half two mode in phase) at $25 \mathrm{~ns}$. The spikes corresponding to the long-wavelength mode are just beginning to break up all along the interface. Interpretation of the data is difficult because the single-mode side of the target may have been corrupted by spike breakup on the two-mode side or by the discontinuity at the boundary between the two sides. In addition, the discontinuity at the boundary makes observations near the centerline unreliable, and it is precisely this region that is least affected by interface curvature and therefore generally the source of the best data.

Because of these factors, the second target series (shot in August 2003) included two single-mode targets (imaged at 18 and $25 \mathrm{ns)} \mathrm{and} \mathrm{two} \mathrm{two-mode} \mathrm{out-of-}$ phase targets (at 25 and $30 \mathrm{~ns}$ ). Data obtained at $25 \mathrm{~ns}$ is shown in Figs. 11(b)-11(c) to provide a comparison between the single-mode and the two-mode cases. The single-mode spike and bubble structure initially present in the single-mode target has persisted to late times, while any such structure initially present in the two-mode out of phase target has vanished. As in the simulation, the presence of the shortwavelength mode appears to have completely broken up the primary mode. The uniformity of the x-ray transmission through the mix layer suggests that 3D structure has emerged and a transition to turbulence may have taken place. 


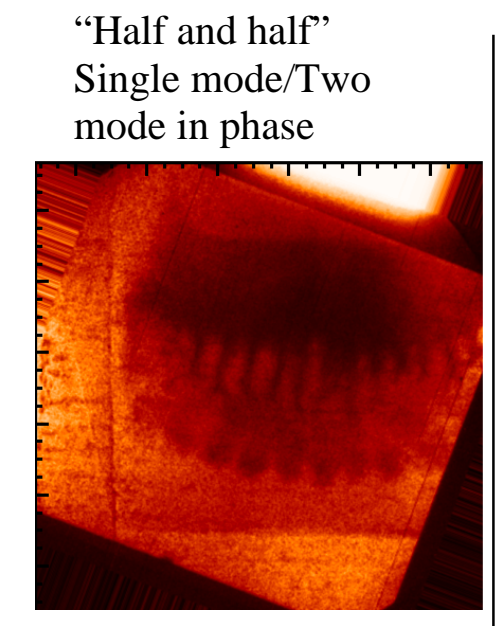

Apr. 25, 2003

(a)

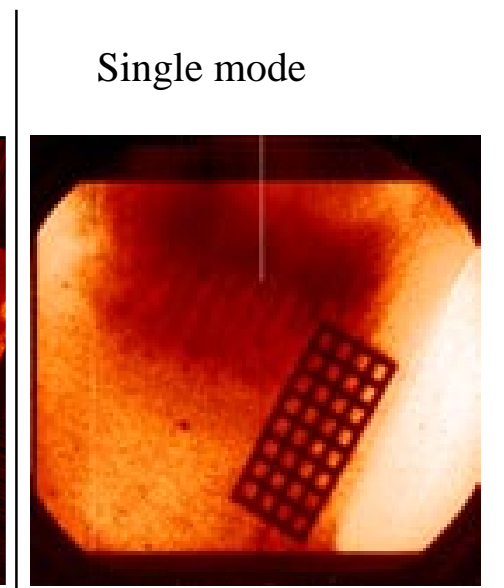

Signal $/$ noise $=1.146$
Two mode out of phase

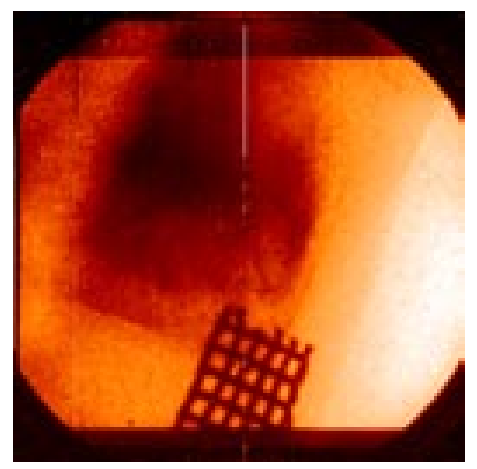

Aug. 21, 2003

Signal $/$ noise $=1.124$

(b)

Figure 11: Experimental radiographs at 25 ns. (a) A "half and half" target with a single mode perturbation on one side and two mode in phase on the other exhibits single-mode-like spike and bubble structure that is just beginning to break down. (b) The single mode target retains single-mode-like spike and bubble structure, while the (c) two mode out of phase shows reduced growth and a breakdown of large-scale structures.

Mix width data from all of the shots is compared in Fig. 12 with the simulation results, in this case shown without subtraction of the decompression effect. The data points are shifted by $1 \mathrm{~ns}$ to account for the way in which the simulations are initiated, and the experimental error is given by the height of the data-point boxes. Two data points are included for each half single-mode/half two-mode - one for each side of the target. Below $20 \mathrm{~ns}$, all three simulations agree to within the experimental error, and the data agree with the simulations. The single-mode and in-phase calculations agree with each other above $20 \mathrm{~ns}$ as well, while the predicted out-ofphase amplitude falls significantly lower. Data is available for all three cases at $25 \mathrm{ns,}$ but the predicted amplitude reduction is still comparable to the measurement 


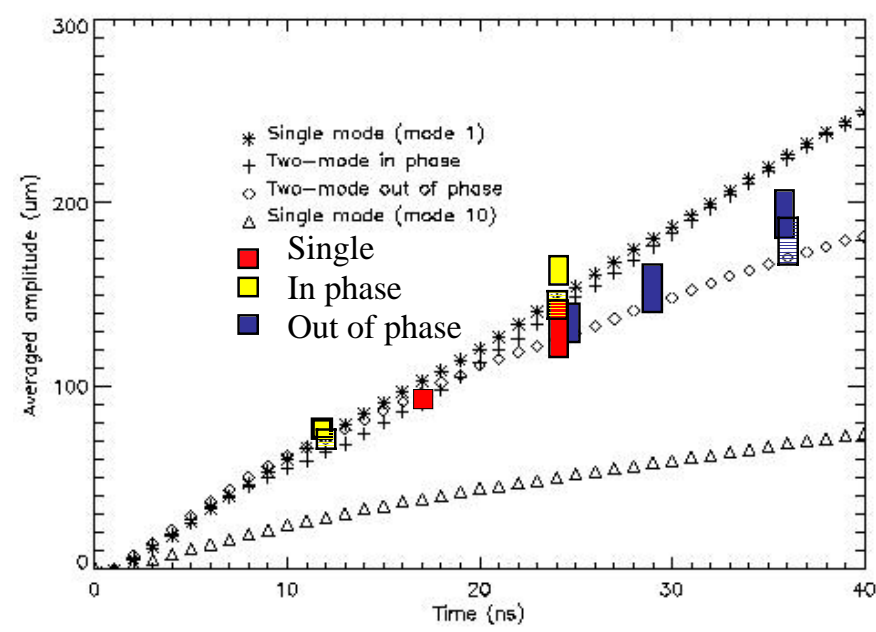

Figure 12: Comparison of data with simulation without decompression effect subtraction. The size of the data points is the size of the error bars. Two data points are included for each "half and half" targets, one from the single mode side and one from the two mode side. Since it is uncertain which side is which, both points are labeled either in phase or out of phase. As predicted by the simulations, the two mode out of phase targets exhibit reduced growth relative to the single mode. The late-time behavior of the two mode in phase case cannot be resolved by the data.

uncertainty. Above $25 \mathrm{~ns}$, available data is limited to an out-of-phase point at $30 \mathrm{~ns}$, which agrees with the simulation, and two points from a half single-mode/half out-ofphase target at $37 \mathrm{~ns}$. The smaller-amplitude side of the target agrees well with the two-mode out of phase simulation, while the larger-amplitude side falls between the predicted single mode and out-of-phase amplitudes. A possible explanation is that the larger-amplitude side is the single mode perturbation corrupted by the out-of-phase side.

Though the quality and quantity of the data are not sufficient to definitively validate the simulation results, they do confirm that the presence of the secondary mode can dramatically alter the evolution of the mix region in the late nonlinear regime. They also suggest that the breakup of the large-scale spikes, which almost certainly indicates the presence of 3D flow and might correspond to a transition to 
turbulence, results in a large reduction in the growth of the mix region relative to the single-mode result. In this case, the reduced amplitude is close to the prediction of the two-mode out-of-phase simulation.

\section{Analysis of earlier results}

In a previous paper, ${ }^{15}$ we presented simulations of two and eight mode experiments performed on the OMEGA laser. The 2-mode perturbation [see Fig. 13(a)] was characterized by a sum of two sinusoidal components (in phase) with wavelengths of 40 and $60 \mu \mathrm{m}$ and amplitudes of 1.25 and $1.5 \mu \mathrm{m}$, respectively. The wavelengths present in the eight-mode case are given by $\lambda_{i}=180 \mu \mathrm{m} / i$ with $i$ ranging from 1 to 8 , while the amplitudes range from 0.4 to $0.7 \mu \mathrm{m}$. As in the two-mode case, all modes in the eight-mode case were in phase. We found that CALE simulations agreed well with the experimental radiography in perturbation amplitude and interface structure. However, despite efforts to account for bubble merger, we were unable to successfully apply the buoyancy-drag model that had successfully predicted the single-mode growth.

In the two-mode case, the bubble growth is consistent with the model description. The spike velocity, on the other hand, abruptly increases at about $15 \mathrm{~ns}$ and subsequently undergoes a period of growth that is nearly linear in time. This can now be understood in the context of the secondary spike interaction and jet formation process. A pair of secondary spikes with both transverse and upstream growth components is clearly visible near the tip of every other primary spike at $13 \mathrm{~ns}$ in both 


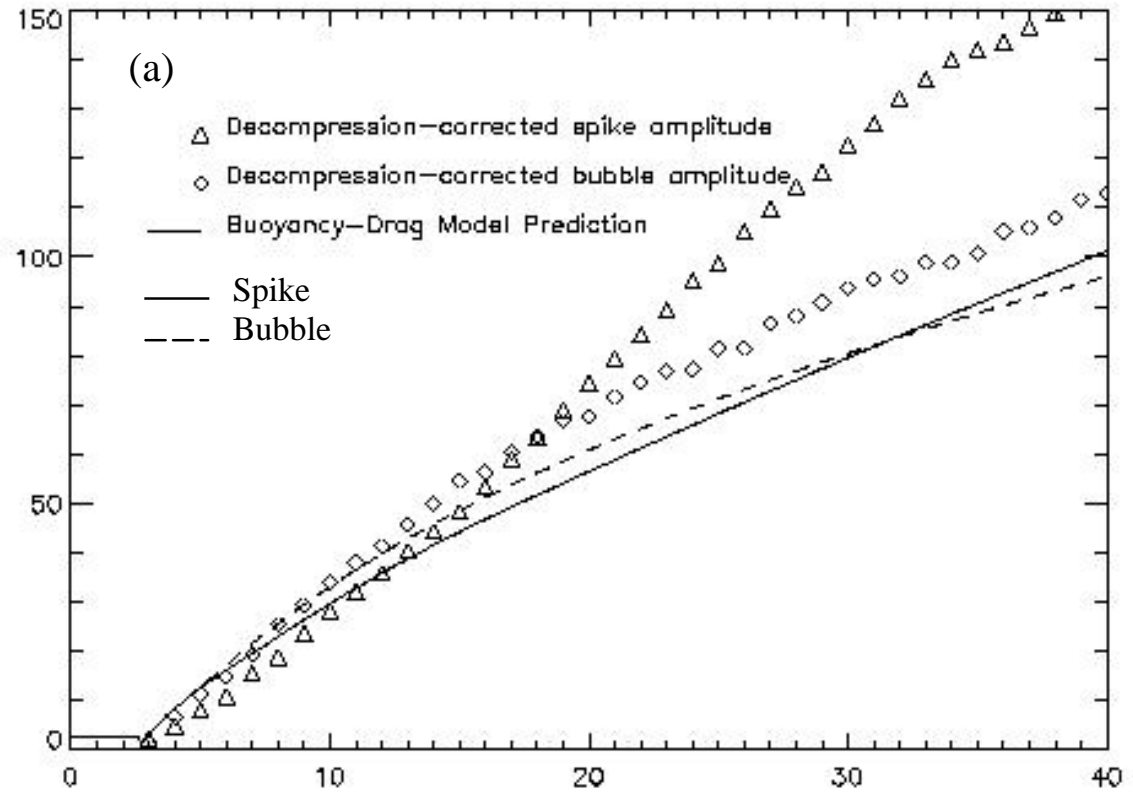

(b)

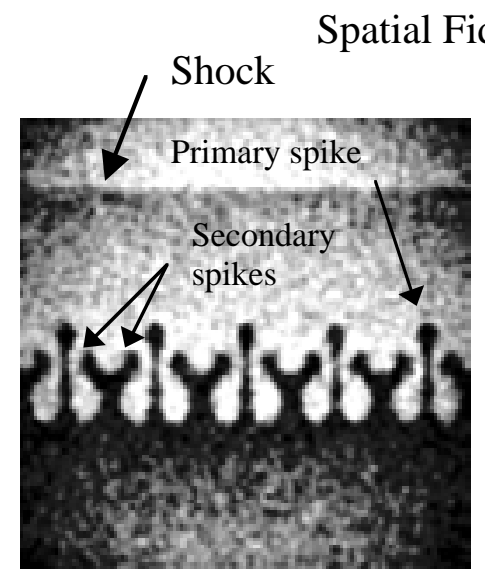

Simulated Radiograph

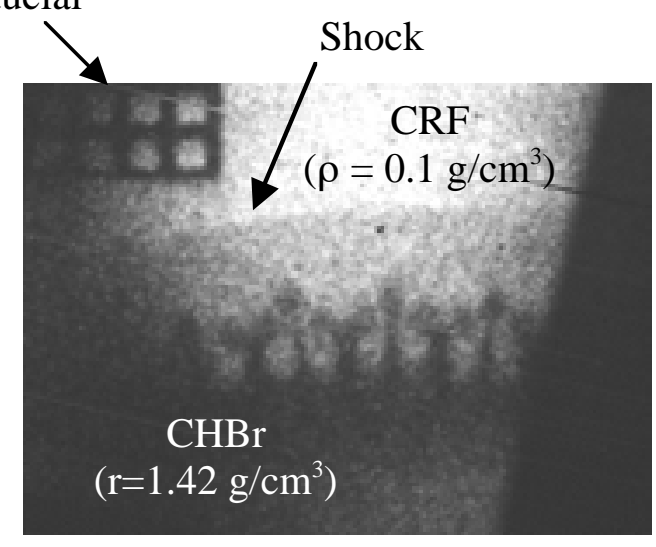

Experimental Radiograph

$$
\mathrm{T}=13 \mathrm{~ns}
$$

Figure 13: Previous two mode simulation and experiment show spike growth enhancement due to the interaction of secondary spikes. (a) Decompression-corrected spike and bubble amplitudes. The spike velocity increases suddenly at about $15 \mathrm{~ns}$. (b) Simulated and experimental radiographs at $13 \mathrm{~ns}$, just prior to secondary spike interaction. 
the simulation and experiment (see Fig. 13). In the simulation, the sudden increase in spike velocity occurs when these pairs of secondary spikes strike the intervening primary spikes, to which some of their upstream energy is transferred. Because the secondary spikes do not have a downstream growth component, there is no large downstream jet production and no consequent bubble growth enhancement. In the eight-mode case, the collision of pairs of spikes (the distinction between "primary" and "secondary" is ambiguous because there is less separation of the initial modes in wavenumber space) does create downstream-directed jets that strike the inner bubble surface, resulting in an abrupt increase in bubble velocity at about $18 \mathrm{~ns}$ (see Fig. 14). At late times, the bubble amplitude is significantly greater (by about $30 \%$ ) than that of the spikes.

In both the two and eight mode cases, spike-spike interactions strongly influence the late-time amplitude histories. The exact nature of the effect, however, including the relative influence on spikes and bubbles, depends on the details of the initial spectrum.

\section{$\underline{\text { IX. Discussion }}$}

The aerodynamic drag pressure acting on the developing spikes not only determines their terminal velocity, but also affects their shape. As the instability growth rate increases, so too does the transverse velocity of redirected secondary spikes. The degree to which the large-scale interface structure is influenced when redirected secondary spikes collide with each other or with primary spikes is 


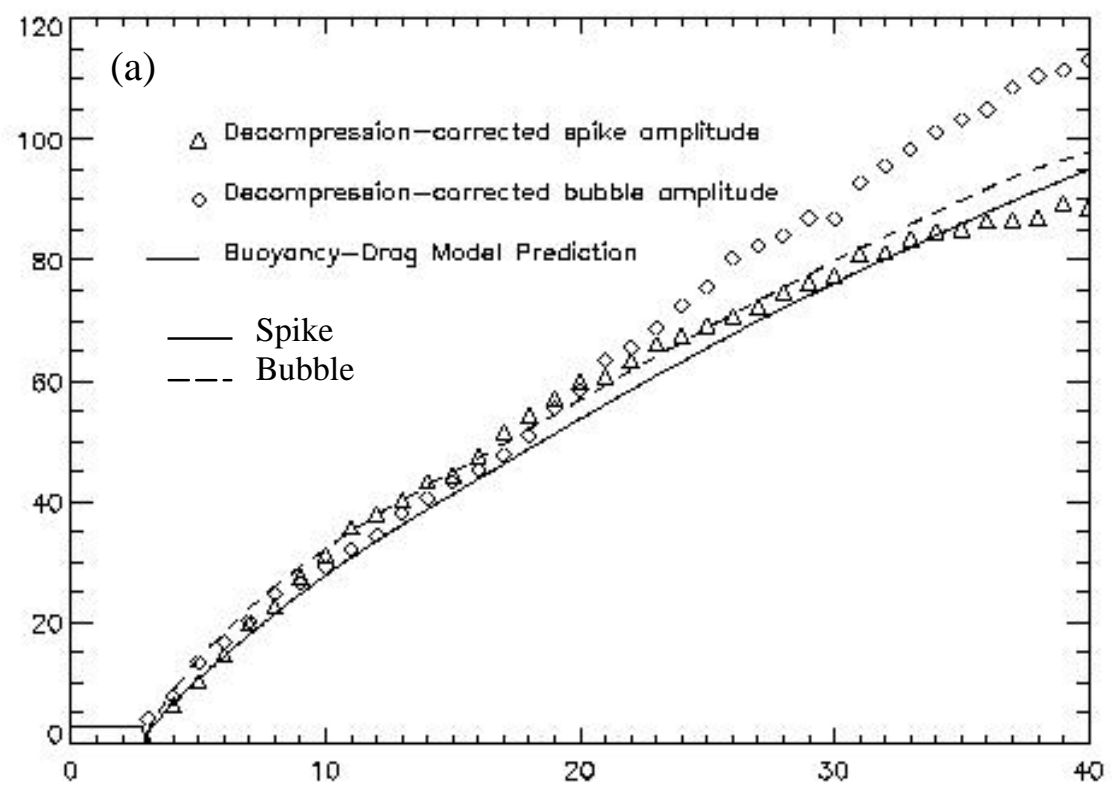

(b)

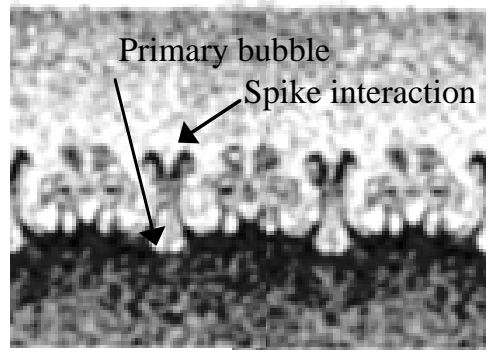

Simulated Radiograph

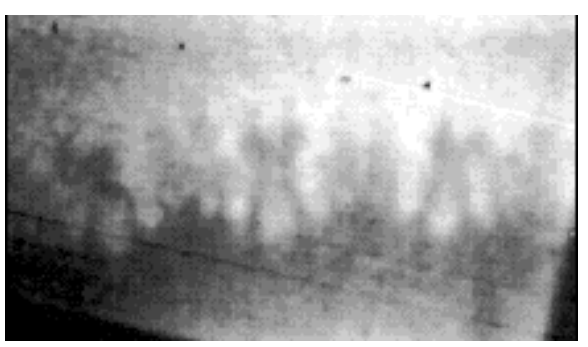

Experimental Radiograph

$$
\mathrm{T}=13 \mathrm{~ns}
$$

Figure 14: Previous eight mode experiment and simulation show bubble growth enhancement due to jet production and interaction with bubbles. (a) Decompressioncorrected spike and bubble amplitudes. (b) Simulated and experimental radiographs at 13 ns showing spike interaction that leads to jet production above the primary bubble tip. 
determined by their speed. The drag pressure is related to the thermal (or interface) pressure $\mathrm{P}_{\mathrm{i}}$ through the expression

$$
\rho_{s}^{*} v_{s}^{2}=\gamma_{s} M^{2} P_{i}
$$

where $\mathrm{M}$ is the instability Mach number, $\mathrm{v}_{\mathrm{s}}$ is the spike velocity, and $\rho_{\mathrm{s}}$ and $\gamma_{\mathrm{s}}$ are the density and adiabatic index of the spike material. When the Mach number is equal to $0.3\left(\mathrm{M}^{2} \approx 0.1\right.$ is the threshold for the appearance of compressibility effects $\left.{ }^{33}\right)$, the drag pressure is about one-tenth of the thermal pressure. Instability Mach numbers in our case in fact approach this regime (recall $\mathrm{M}_{\text {foam }}=0.15-0.20$ and $\mathrm{M}_{\text {plastic }}=0.25-0.40$ ). The process whereby the local pressure increase in secondary bubble regions results in partially redirecting the growth of secondary spikes into the transverse direction is reminiscent of the pinching effect noted by Li to occur during bubble merger. ${ }^{34}$ Because the flow velocity into the primary bubble is greater than into the secondary bubble, Bernoulli's equation requires that the pressure there be lower. Consequently, the spike dividing the two regions is redirected towards the primary bubble's center. Li hypothesized that the resulting reduction in effective Atwood number would eventually reduce the velocity of the larger bubble. But in our in-phase case, the headon collision of two fast-moving secondary spikes produces an even faster downstream-directed jet. When it strikes the inner surface of the primary bubble, its ram pressure in the bubble frame is $0.45 \mathrm{Mbar}$ - roughly equal to the $0.50 \mathrm{Mbar}$ thermal pressure of the plastic at the bubble position. It is therefore able to penetrate a significant distance into the plastic (in a process similar to a cratering event), thereby enhancing the bubble growth. Although there is no head-on collision of 
secondary spikes in the out-of-phase case, the redirected spikes are still sufficiently energetic to essentially punch through the primary spike stalks.

Whether or not redirected secondary spikes collide with each other or with primary spike stalks depends critically on the degree of phase coherence. Interfaces consisting of periodic arrays of spikes are more likely to evolve into colliding spikes. For a system such as ours with two commensurate modes, this requirement is satisfied when the modes are in phase. Of course real physical systems such as interfaces within supernovae and ICF targets, whether characterized by only a few or by many modes, are very unlikely to exhibit high degrees of phase coherence. Indeed, the perfect symmetry enforced by the in-phase calculation is arguably rather unphysical. Systems comprised of incommensurate modes can, however, have regions of locally significant phase coherence. Such regions will likely exhibit accelerated growth, but whether this eventually determines the dominant scales of the mix region remains to be seen.

In addition, there are important implications for those who wish to model physical systems. In multimode RT and RM simulations, the domain is often limited to a subsection or wedge of the full system with reflecting boundary conditions. In order to avoid unphysical effects at the boundaries, the initial perturbation spectrum sometimes includes only modes whose wavelengths are integer fractions of the full domain. The "random phase" assignment then amounts simply to a random assignment of plus or minus one to the amplitude of each mode. Since such spectra are actually characterized by a high degree of phase coherence, these simulations, if strongly driven, might significantly over-predict the growth of the mixing layer. 
Regardless of the degree of phase coherence, the interaction of redirected spikes represents a coupling between transverse and parallel motions and a complicated nonlinear transfer of energy from spikes to other spikes (driving premature bubble merger) and to bubbles (perhaps resulting in increased spike-bubble symmetry). Coupling of this process with the KH instability results in additional coupling between and generation of scales and greatly enhanced mixing in the layer. Since turbulence requires the development of a broad inertial range of scales, this will likely decrease the time to transition.

The result that large-scale features present during the late nonlinear instability evolution are strongly affected by details of the initial conditions must be reconciled with the expectation that, at some point near or after transition, the mixing layer will begin to grow at a rate that is independent of the initial spectrum. If this expectation is correct, then the memory of the initial conditions must somehow be erased. The observed dependence would in that case be a transient phenomenon that would eventually disappear as the bubble size distribution settles into a scale invariant form. However, this argument requires the continual emergence of larger scales and depends on the existence of a sustained drive. In our case, the acceleration is decaying as the shock moves continually further away from the interface. At the same time, perturbation growth continues because of material decompression. The combination of a decaying drive and continuing decompression means that transients can effectively be "frozen in" to the flow and thereby persist to late times. 


\section{$\underline{X . \text { Conclusions }}$}

In conclusion, we have shown one way in which compressibility affects the 2D evolution of hydrodynamically unstable interfaces. When the instability Mach number is higher than 0.1 , the pressure associated with kinematic drag on developing spikes can be sufficient to partially redirect spike growth into the transverse direction. For an interface with a long-wavelength primary mode and a short wavelength secondary mode, the interaction of redirected secondary spikes introduces sensitivity to the initial conditions (both the spectrum and phase distribution). The developing instability then "remembers" small-scale details of the IC's well into the late nonlinear stage of its evolution. Phase coherence tends to result in increased perturbation growth - especially of bubbles, which could lead some "random phase" multimode simulations to overstate the growth of the modeled physical system. Phase-decoherent noise generally results in growth suppression. Coupling of this process with the KH instability results in additional coupling between and generation of scales and greatly enhanced mixing in the layer.

Demonstrating the transition to turbulence in high Mach number experiments is a crucial step in developing an experiment-based understanding of supernova hydrodynamics. Nevertheless, in order to truly represent the desired astrophysical system, future experiments will need to not only reach transition, but to reach it early on while the acceleration is still large. This will likely require a significant increase in the drive intensity, and would also benefit from 3D IC's consisting of many short 
wavelength modes. With this in mind, we are beginning to use adaptive mesh refinement (AMR) simulations with NIF-like drive conditions to investigate the dependence on the spectrum and degree of phase coherence for interfaces with many modes over a wide range of scales. Finally, we note that we expect the jet effect to be significantly smaller, if not altogether absent, in 3D systems with broad spectra.

Consequently, 3D calculations are being planned to study the effect of initial conditions on determining the time to transition and the properties of the subsequent turbulent flow. 


\section{Chapter 4: Bubble merger model for the nonlinear}

\section{Rayleigh-Taylor instability driven by a strong blast wave}

\section{Introduction}

The basic Rayleigh-Taylor (RT) instability criterion, ${ }^{1,2}$ neglecting certain potentially stabilizing factors such as surface tension ${ }^{3}$, is the existence of anti-parallel components of pressure and density gradients $(\nabla \mathrm{P} \bullet \nabla \rho<0)$. When this condition is met at an interface between two materials with density ratio $\eta<1$, perturbations on the interface will grow in time. In the inviscid limit, the instability develops exponentially while the perturbations remain small (during the linear phase) with a growth rate given by ${ }^{4}$

$$
\gamma=\sqrt{\frac{k g A}{1+k L}}
$$

where $\mathrm{k}$ is the perturbation wavenumber, $\mathrm{g}$ is the acceleration, $\mathrm{A}=(1-\eta) /(1+\eta)$ is the Atwood number, and $\mathrm{L}$ is the density gradient scale length at the interface. At later times, initially sinusoidal perturbations grow into spikes of heavier fluid "falling" into lighter fluid and bubbles of lighter fluid "rising" into heavier fluid. For $A=1$, the bubbles rise with constant (terminal) velocity while spikes fall with constant acceleration in the nonlinear regime. ${ }^{5,6}$ When $\mathrm{A}<1$, the spike eventually also reaches terminal velocity. ${ }^{6}$ 


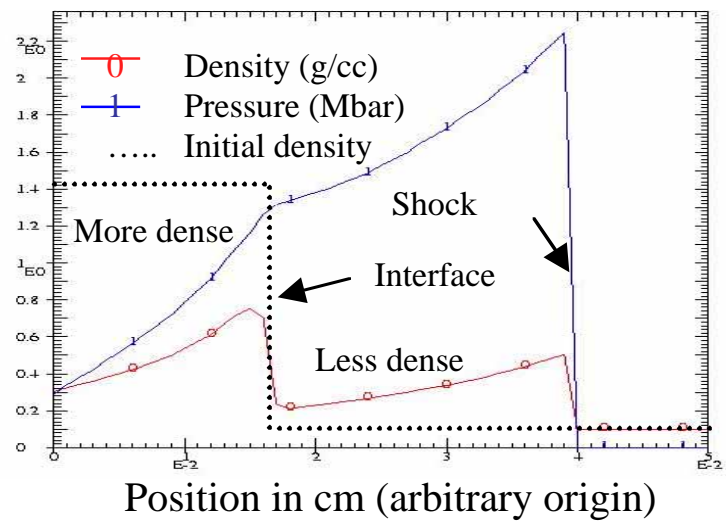

Figure 1: Density (curve 0) and pressure (curve 1) behind a blast wave that has passed through a material interface from a $1.42 \mathrm{~g} / \mathrm{cc}$ plastic to a $0.1 \mathrm{~g} / \mathrm{cc}$ foam. The interface is RT unstable due to the presence of antiparallel density and pressure gradients at the interface. The dotted line shows the initial (pre-shock) density profile. The data are from a 1D CALE simulation of the experiment-relevant planar hydrodynamics at 14 ns.

The RT instability criterion can also be satisfied at a material interface through which a blast wave has been transmitted from a heavier to a lighter fluid. ${ }^{8}$ As is illustrated in Fig. 1, since the pressure behind a blast wave is always falling in time at any fixed point and in distance behind the shock front (at least in the self-similar regime $^{9}$ ), an interface generally becomes RT unstable when it transmits a blast wave down a density gradient (ie from more dense to less dense material).

Shock and blast waves are common participants in astrophysical processes. ${ }^{10}$ In a core-collapse supernova, for example, the sudden release of an enormous amount of energy at the star's core drives a strong blast wave that propagates out through layers of progressively less dense matter. ${ }^{11,12}$ As the interfaces between these layers subsequently decelerate in the expansion fan behind the blast front, they are RT unstable. ${ }^{8}$ The potential significance of this phenomenon was realized with observations of SN1987A, when it was found that spherically-symmetric explosion 
models failed to correctly predict the velocity and arrival time at the surface of heavy elements originating from the star's central regions. It has been suggested that the discrepancy results from the failure of the 1D models to account for the turbulent (the Reynolds number has been estimated ${ }^{13}$ to be of order $10^{10}$ ) RT mixing that is certainly occurring at unstable interfaces. ${ }^{11,12,14,15}$

A great deal of experimental, theoretical, and computational work has been directed towards understanding the evolution of RT unstable systems, and much progress has been made in the last fifty years. Much of this effort has focused on the classical case of incompressible fluids in a uniform acceleration field. For systems driven by strong blast waves, such as core-collapse supernovae and the high-energydensity laser-driven experiments ${ }^{16-24}$ designed to study them, the acceleration is strongly time-dependent and the flow is compressible. Consequently, ideas and observations pertaining to classical RT systems do not necessarily apply. The purpose of this paper is to examine how models of nonlinear RT growth should be modified when applied to blast-wave-driven systems and what implications these differences have on self-similarity, loss of initial conditions, and transition to turbulence.

We begin by reviewing the stages of classical RT instability growth, focusing on the nonlinear regime, and describe the types of models commonly used to predict the evolution of multimode interfaces. We use a simple version of the Sharp-Wheeler bubble merger model ${ }^{25,26}$ to illustrate the loss of memory of initial conditions and the hypothesized establishment of a self-similar regime, and discuss their relationship to the system's transition to turbulence. After a brief review of relevant aspects of blast wave theory, we outline in more detail the peculiarities of blast-wave-driven RT and 
present an appropriately generalized buoyancy-drag model. We discuss bubble merger for the blast-wave-driven case and the statistical-mechanical merger model resulting from combining it with the single-mode buoyancy-drag model.

For planar blast-waves, the onset of self-similar instability growth and the loss of memory of initial conditions do not follow from the model as they do in the classical case. Instead, the ratio of mix width to dominant transverse scale grows slowly in time during what we call a quasi-self-similar regime.

Self-similarity and loss of memory of initial conditions are possible for divergent systems, but require very high initial characteristic mode numbers and high incident Mach numbers. This requirement has serious implications for supernovae. Initial conditions predicted by recent stellar calculations ${ }^{27.28}$ suggest that initial mode numbers present in supernova progenitors are not high enough to reach the selfsimilar regime. Instead, the late-time instability evolution would depend on the initial perturbation spectrum.

Finally, we include a generalization of the model for interfaces driven by nonideal blast waves and make some comparisons with simulations, experiments, and observations.

\section{Classical case}

In its most basic formulation, the Rayleigh-Taylor instability describes the evolution of an interface separating a heavier (more dense) fluid supported by a 
lighter (less dense) fluid in a uniform acceleration field. In the linear approximation, which is valid for ka $<1$, pertubation growth is exponential with a growth rate given by Eq. (1). When the amplitude becomes comparable to the wavelength, the linear approximation breaks down. Buoyancy-drag models treat bubbles of lighter fluid and spikes of heavier fluid as the fundamental nonlinear objects. The model of Oron $e t$ $a l,{ }^{29}$ which follows earlier work by Youngs ${ }^{30}$ and Dimonte and Schneider,${ }^{31}$ predicts that $2 \mathrm{D}$ bubbles "rise" with a velocity determined by

$$
\left(\rho_{b}+C_{a} \rho_{s}\right) \frac{d u_{b}(t)}{d t}=\left(\rho_{s}-\rho_{b}\right) g(t)-\frac{C_{D}}{\lambda} \rho_{s} u_{b}^{2}
$$

The equation for the spike velocity is obtained by simply interchanging spike and bubble densities. The added mass coefficient $\mathrm{C}_{\mathrm{a}}$ equals 2 for $2 \mathrm{D}$ perturbations and 1 for $3 D$ perturbations, while the drag coefficient $C_{D}$ is $6 \pi$ for $2 D$ perturbations and $2 \beta_{1}$ $\approx 7.66 \approx 1.22 \times 2 \pi$, where $\beta_{1}$ is the first zero of the first-order Bessel function, for $3 \mathrm{D}$ perturbations. Some authors, including Oron et al., ${ }^{29}$ use $C_{D}=2 \pi$ for 3D bubbles. These drag coefficients, originally derived by Layzer ${ }^{32}$ following earlier work by Davies and Taylor, ${ }^{33}$ apply to $\mathrm{A}=1$ bubbles rising in tubes of diameter $\lambda$ or $2 \mathrm{D}$ flow between parallel plates. In either case, $\lambda$ is twice the transverse scale of the bubble. Since spikes at $A=1$ are infinitely narrow, the transverse scale of the bubble is equal to the wavelength of a periodic array of bubbles. As A decreases below unity, however, the width of the spikes increases until, at $\mathrm{A}=0$, there is no distinction between spikes and bubbles. Rather than making the transverse scale in Eq. (2a) a function of the perturbation wavelength and Atwood number, we instead redefine the drag coefficients so that 


$$
\begin{aligned}
C_{D}{ }^{2 D} & =\left\{\begin{array}{ll}
3 \pi(1+A) & \text { for bubbles } \\
3 \pi(1-A) & \text { for spikes }
\end{array}\right\} \\
C_{D}{ }^{3 D} & =\left\{\begin{array}{ll}
\beta_{1}(1+A) & \text { for bubbles } \\
\beta_{1}(1-A) & \text { for spikes }
\end{array}\right\}
\end{aligned}
$$

With this definition, $\lambda$ is always the perturbation wavelength. Because of KelvinHelmholtz $(\mathrm{KH})$ rollup at the spike tips for $\mathrm{A}<1$, the actual spike width will be greater than that implied by (2b). The same is true for bubbles at low Atwood number. In those cases where $\mathrm{KH}$ effects have a significant impact on the spike and/or bubble width, the drag coefficients should be adjusted accordingly.

Strictly speaking, the values in (2a) apply to single bubbles that are laterally confined or periodic bubble arrays. For chaotic bubble fronts, experiments ${ }^{34}$ and simulations ${ }^{35}$ suggest that the drag coefficients should be smaller by a factor of about $6 \pi$, corresponding to asymptotic velocities that are higher by a factor of about 2.5 . Glimm and Li have suggested that this is because the leading bubbles in chaotic arrays are laterally less confined by their neighbors then those in periodic arrays. ${ }^{26}$ Equation (2a) is just Newton's second law, where the inertial term on the left of the equals sign contains an added mass coefficient $\mathrm{C}_{\mathrm{a}}$ and the two forces (per unit volume) on the right-hand side are buoyancy and drag. Terminal velocity is attained when the two forces balance one another, and the resulting asymptotic velocity is

$u_{b, s}^{a s y m}=\sqrt{\frac{\left|1-\eta_{b, s}\right|}{C_{D}} L g} \equiv C_{b, s} \sqrt{L g}$,

where $\eta_{\mathrm{b}, \mathrm{s}}=\rho_{\mathrm{b}, \mathrm{s}} / \rho_{\mathrm{s}, \mathrm{b}}$. In what follows, we typically drop the spike and bubble subscripts with the understanding that the merger models describe mergers between 
bubbles rather than spikes. Merger models are based on the fact that larger bubbles rise (or grow) faster than smaller bubbles and the observation that smaller bubbles "merge" to form larger bubbles. Statistical mechanics merger models for the Rayleigh-Taylor instability describe the evolution of a bubble size distribution function $g(\lambda, t)$, which gives the number of bubbles in the system with transverse sizes between $\lambda$ and $\lambda+d \lambda$ at time $t^{26,36}$ Such models are built out of two main components. The first component is a model for the velocity of a single bubble, such as Eq. (2) or, more typically, Eq. (3), and the second is a rule for the merger of two neighboring bubbles. Merger rates have been obtained from theory, ${ }^{26,32,36-39}$ simulation, ${ }^{26,40}$ and experiments. ${ }^{26}$ In the very simplest model, the merger rate is a constant independent of the size of the bubbles involved. ${ }^{36}$ In a more reasonable approach, which we will adopt, two neighboring bubbles are said to have merged when the larger of the two has risen above the smaller bubble by a constant fraction of the smaller bubble's transverse size. ${ }^{26,37}$ In either case, the smaller bubble, observed experimentally ${ }^{34}$ to be "washed down stream", is removed from the ensemble. In 2D, the diameter of the surviving bubble is equal to the sum of the diameters of the two pre-merger bubbles. In $3 \mathrm{D}$, area rather than diameter is the conserved quantity.

The evolution of the bubble-size distribution $\mathrm{g}(\mathrm{L}, \mathrm{t})$ is given by ${ }^{36}$ $N(t) \frac{d g(L, t)}{d t}=-2 g(L, t) \int_{0}^{\infty} g\left(L^{\prime}, t\right) \omega\left(L, L^{\prime}\right) d L^{\prime}+\int_{0}^{\infty} g\left(L^{\prime}, t\right) g\left(L-L^{\prime}, t\right) \omega\left(L^{\prime}, L-L^{\prime}\right) d L^{\prime}$

where the total number of bubbles $N(t)=\int_{0}^{\infty} g(L, t) d L$ and the merger rate $\omega\left(\mathrm{L}, \mathrm{L}^{\prime}\right)$ is inversely proportional to the time interval required for a merger between two adjacent bubbles with transverse scales L and L'. The first term on the right-hand side is the 
rate at which bubbles of size L are lost in merger event, and the second term gives the rate at which bubbles of size $\mathrm{L}$ are generated.

It has been proposed that, at least for the classical case, an RT-unstable system tends to approach a self-similar (or scale-invariant) regime independent of the initial conditions after several generations of bubble merger above the largest significant scales present in the initial spectrum. ${ }^{41}$ This is true of the model given in Eq. (4) for a wide range of merger rates, and is also supported by a large body of experimental, ${ }^{34}$ theoretical, ${ }^{37,42}$ and computational ${ }^{41}$ work. Late-time independence of initial conditions in hydrodynamically-unstable systems means that two interfaces, even if characterized by wildly different perturbation spectra at time zero, will eventually reach a regime in which their perturbation amplitudes, velocities, and statistical spectral properties (or bubble size distribution functions) will become equalized. Strictly speaking, we require that the relative difference between the two systems vanishes in the limit that $\mathrm{t}$ goes to infinity. The instability evolution is self-similar if the shape of the bubble distribution function does not change in time except for a linear multiplier that increases in proportion with the bubble size expectation value. In the self-similar regime, the mix width $\mathrm{h}(\mathrm{t})$ grows in proportion to the characteristic bubble size (the bubble size expectation value).

To illustrate the mechanism by which initial conditions might be erased in the RT instability, consider the following toy model of bubble merger: Assume there is only one bubble size (L) at any time $\mathrm{t}$, and that $\mathrm{L}_{0}$ is the bubble size at time zero. Then, at each merger, the bubble size is doubled, so we have

$$
\mathrm{L}(\mathrm{t})=2^{\mathrm{N}(\mathrm{t})} \mathrm{L}_{0}
$$


where $N(t)$ is the number of bubbles of size $L$ at time t. Now $N(t)$ is determined by the requirement that

$$
\mathrm{t}=\tau\left(\mathrm{L}_{0}\right)+\tau\left(2 \mathrm{~L}_{0}\right)+\ldots+\tau\left(2^{\mathrm{N}(\mathrm{t})} \mathrm{L}_{0}\right)
$$

where $\tau(L)$ is the merger time for a bubbles of size L. If we had $\tau(L)=\tau$ independent of $L$, then $N(t)=t / \tau$, and so $L$ would grow exponentially in time. Loss of initial conditions is possible because the merger rate is not independent of bubble size. Instead, larger bubbles take longer to merge than smaller bubbles. For classical RT, dimensional analysis requires that ${ }^{25}$

$$
\tau(L) \propto \sqrt{L / g} .
$$

The terminal velocity for bubbles of size $\mathrm{L}$ (and spikes for $\mathrm{A}<1$ ) is given by Eq. (3). The coefficient $\mathrm{C}$ depends on the dimension of the perturbation and the Atwood number. Note that Eq. (7) says that the bubble merger time is proportional to the time it takes the bubble to rise by one bubble diameter. We define the dimensionless constant $\mathrm{C}_{2}$ as the fraction of a bubble diameter the bubble must rise before merger takes place, so that

$$
\tau(L)=\left(C_{2} / C\right) \sqrt{L / g}=\left(C_{2} / C\right) \sqrt{L_{0} / g} 2^{i / 2}
$$

where $\mathrm{i}$ is the bubble generation number. Requirement (6) then becomes

$$
t=\frac{C_{2}}{C} \sqrt{\frac{L_{0}}{g}} \sum_{i=0}^{N} 2^{i / 2}=\frac{C_{2}}{C} \sqrt{\frac{L_{0}}{g}} \frac{\sqrt{2} 2^{N / 2}-1}{\sqrt{2}-1},
$$

from which it follows that

$$
N(t)=2 \log \left[\frac{C}{C_{2}}(\sqrt{2}-1) \sqrt{\frac{g}{L_{0}}} t+1\right]-1
$$

Inserting this into (5), we finally get 


$$
L(t)=\left[\frac{C}{C_{2}}\left(1-\frac{1}{\sqrt{2}}\right) \sqrt{g} t+\sqrt{\frac{L_{0}}{2}}\right]^{2} .
$$

For times such that the inequality

$$
t>>\frac{C_{2}}{C} \sqrt{\frac{L_{0}}{g}} \equiv \tau\left(L_{0}\right)
$$

is satisfied, the first term in the square brackets dominates, and we get the limiting result

$$
L(t) \rightarrow\left[\frac{C}{C_{2}}\left(1-\frac{1}{\sqrt{2}}\right)\right]^{2} g t^{2}
$$

That is, the dependence on initial conditions (the initial bubble size $\mathrm{L}_{0}$ ) is lost at times long compared to the first generation bubble merger time. Consequently, $\mathrm{gt}^{2}$ is the only transverse scale remaining in the problem.

Complete scale invariance follows from a similar argument. If we assume that the merger process begin only after the $\mathrm{L}_{0}$-size bubbles have reached their saturation velocity, then we can express the perturbation height $h(t)$ as the sum of the height at the saturation time $\mathrm{h}_{\text {sat }}$ plus the sum of the contributions of each generation:

$$
h(t)=h_{\text {sat }}+\sum_{i=0}^{N} u_{i}^{\text {asym }} \tau_{i}=h_{\text {sat }}+C_{2} L_{0}\left(2^{N+1}-1\right) .
$$

Using (5) and (9), this can be written as

$$
h(t)-h_{s a t}=C_{2}\left(2 L(t)-L_{0}\right) .
$$

With the solution for L(t) in Eq. (11), this becomes

$$
h(t)-h_{s a t}=(\sqrt{2}-1)^{2} \frac{C^{2}}{C_{2}} g t^{2}+C(\sqrt{2}-1) \sqrt{L_{0} g} t .
$$

When condition (12) is satisfied, we are left with 


$$
h(t)-h_{s a t}=\alpha(A) A g t^{2}
$$

where we have defined

$$
\alpha(A)=(\sqrt{2}-1)^{2} \frac{C^{2}}{C_{2} A} .
$$

Since $h_{\text {sat }}$ is of order $L_{0}$, it too can be neglected when (12) is satisfied if $C_{2}$ is greater than or of order unity. This yields the familiar result

$$
h(t)=\alpha(A) A g t^{2}
$$

Thus at late times the initial scale $\mathrm{L}_{0}$ is not retained in the expression for the perturbation height. Together, Equations (13) and (19) show that $\mathrm{gt}^{2}$ emerges as the only remaining length scale in the problem. Since $L(t)$ and $h(t)$ have the same temporal scaling, the perturbation height is proportional to the wavelength, with

$$
\mathrm{h}(\mathrm{t}) / \mathrm{L}(\mathrm{t})=2 \mathrm{C}_{2} \text {, }
$$

and the system is said to be in the scale invariant regime. Note that asymptotic selfsimilarity follows directly from Eq. (15) and, for our general merger model [Eq. (7)], depends only on the assumption that merger occurs after the bubble has traversed a constant fraction of its diameter.

The mechanism for the loss of initial conditions is worth restating and can be easily visualized as follows. We rewrite Eq. (9) as follows to express the time at which the scale $\mathrm{L}$ is reached:

$$
t(L)=\frac{C_{2}}{C} \sqrt{\frac{L}{g}} \sum_{i=0}^{N} \frac{1}{2^{i / 2}}
$$

where

$$
\mathrm{N}=\log _{2}\left(\mathrm{~L} / \mathrm{L}_{0}\right)
$$

For large N, the sum in Eq. (21) is dominated by the first several terms, while the 


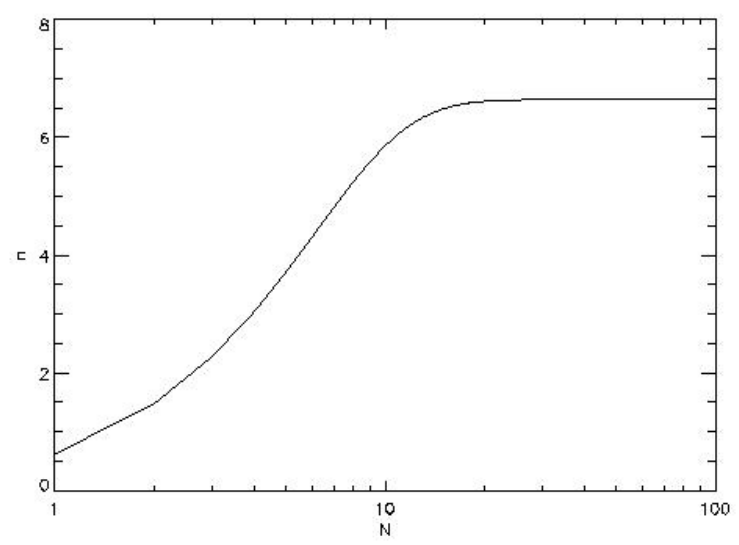

Figure 2: Loss of memory of the initial transverse scale: For a system having undergone $\mathrm{N}$ bubble merger generations, the number of generations $\mathrm{n}<\mathrm{N}$ necessary to include in the sum in Eq. (21) in order to reduce the error relative to the full sum (from 0 to $\mathrm{N}$ ) to $10 \%$. Above $\mathrm{N}=10$, the sum is dominated by the last seven terms.

later terms are of less relative importance. The relative error of a partial sum from $\mathrm{i}$ equals zero to $\mathrm{n}<\mathrm{N}$ is given by

Relative error $=\frac{2^{(N-n) / 2}-1}{\sqrt{2} 2^{N / 2}-1}$.

Figure 2 is a plot of the $\mathrm{n}$ at which the sum is $90 \%$ converged, as a function of $\mathrm{N}$. Above $\mathrm{N}=10$, the the sum is dominated by the last seven terms. Thus as long as $\mathrm{N}$ is large, its precise value is not important in determining $t(\mathrm{~L})$. In other words, after many generations of bubble merger, the time to reach a given scale is only weakly dependent on the initial scale.

Before proceeding, there are a few points worth making. First of all, we note that we could have arrived at Eq. (19) by assuming from the beginning that the system eventually becomes self-similar. ${ }^{43}$ That is, we use Eq. (20) to replace L(t) in Eq. (3) with $\mathrm{h}(\mathrm{t})$ and equate $\mathrm{u}^{\text {asym }}$ with $\mathrm{dh} / \mathrm{dt}$. The solution of the resulting first-order ordinary differential equation is again Eq. (19), now with

$$
\alpha(\mathrm{A})=(1 / 4) \mathrm{C}^{2} /\left(\mathrm{C}_{2} \mathrm{~A}\right) .
$$


This result makes no direct assumption about the form of the merger rate and differs from Eq. (18) only in the numerical pre-factor, which is now 0.25 instead of $\approx 0.1716$. It is not surprising that the exact value of the pre-factor depends on whether the bubble diameter varies discreetly, as in deriving Eq. (18), or continuously, as for Eq. (24).

A second point is that for bubbles $\alpha(\mathrm{A})$ depends on A like $1 /(1+\mathrm{A})$, and therefore changes by only a factor of two over the entire range of possible Atwood numbers (neglecting in this instance the Atwood number dependence of our model's drag coefficient). This reasoning does not necessarily apply to spikes, though it must in the limit of vanishing Atwood number (in which case there is no difference between spikes and bubbles). As A approaches unity, $\alpha(\mathrm{A})$ for spikes must also approach unity so that spikes freefall with the acceleration of gravity. The model fails for spikes at $\mathrm{A}->1$, where it predicts that $\alpha(\mathrm{A})$ increases without bound, because it is based on the assumption that the spikes have reached terminal velocity. Putting in reasonable values for the coefficients and assuming the above scaling for the merger time, we find $\alpha(A)=0.036 /\left[C_{2}(1+A)\right]$ for $2 \mathrm{D}$ bubbles and $\alpha(A)=0.097 /\left[C_{2}(1+A)\right]$ for $3 \mathrm{D}$ bubbles [or $0.053 /\left(\mathrm{C}_{2}(1+\mathrm{A})\right)$ in $2 \mathrm{D}$ and $0.142 /\left(\mathrm{C}_{2}(1+\mathrm{A})\right)$ in $3 \mathrm{D}$ with the prefactor in Eq. (24)]. For spikes, the expressions are the same if we make the substitution A -> -A. Despite the simplicity of our model and with $\mathrm{C}_{2} \approx 1$ as reported by Glimm and $\mathrm{Li}^{26}$ these expressions agree to within about a factor of two with experiments and simulations. ${ }^{43}$

Finally, we consider what a more precise expression for the bubble merger time might look like. In a real system, merger events in general involve bubbles of 
different size that satisfy a merger criterion that depends on the dimension of the system. In $2 \mathrm{D}$, the bubble diameter is conserved $\left[\mathrm{L}_{\mathrm{i}+1}=\mathrm{L}_{\mathrm{i}}+\mathrm{L}_{\mathrm{j}} \equiv \mathrm{L}_{\mathrm{i}}+\kappa^{2} \mathrm{~L}_{\mathrm{i}}=\left(1+\kappa^{2}\right) \mathrm{L}_{\mathrm{i}}\right.$ $\left.\equiv \mu \mathrm{L}_{\mathrm{i}}\right]$, while the bubble area is conserved in $3 \mathrm{D}\left[\mathrm{L}_{\mathrm{i}+1}^{2}=\mathrm{L}_{\mathrm{i}}^{2}+\mathrm{L}_{\mathrm{j}}^{2} \equiv \mathrm{L}_{\mathrm{i}}^{2}+\kappa^{4} \mathrm{~L}_{\mathrm{i}}^{2}=(1+\right.$ $\left.\left.\kappa^{4}\right) \mathrm{L}_{\mathrm{i}}^{2} \equiv \mu^{2} \mathrm{~L}_{\mathrm{i}}^{2}\right] \cdot{ }^{25}$ A periodic array of identical bubbles is stable, so the merger time for two bubbles of equal size should actually be infinite. Finally, the expression for the merger time should be symmetric in $L_{i}$ and $L_{j}$. If we again adopt the convention of Sharp and Wheeler that bubble merger occurs when the height of the larger bubble above the top of the smaller bubble is a fraction $\mathrm{C}_{2}$ of the smaller bubble radius, ${ }^{25}$ then we find

$$
\tau\left(L_{i}\right)=\left(C_{2} / C\right) \sqrt{L_{i} / g} /(\kappa-1)
$$

where $\mathrm{L}_{\mathrm{i}}$ is the smaller of $\mathrm{L}_{\mathrm{i}}$ and $\mathrm{L}_{\mathrm{j}}$ and

$$
\kappa=\sqrt{L_{j} / L_{i}} .
$$

This result, which was also derived by Alon et al..$^{36}$ for the Sharp-Wheeler bubble merger model, satisfies the requirements given above for a well-behaved merger time. The simple merger model discussed above has $\kappa=\sqrt{ } 2$, but all of its conclusions remain qualitatively unchanged for any other constant $\kappa$. Following the same procedure outlined above, we find that the transverse scale evolves according to

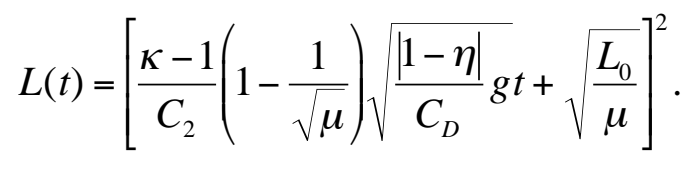

The bubble height growth is given by 


$$
\begin{aligned}
h(t) & =h_{0}+\frac{C_{2}}{\kappa-1}\left(1-\frac{1}{\mu}\right)\left(L(t)-\frac{L_{0}}{\mu}\right) \\
& =\mathrm{h}_{0}+\frac{1-1 / \sqrt{\mu}}{1-1 / \mu} \frac{\kappa-1}{C_{2}}\left(1-\frac{1}{\sqrt{\mu}}\right) \frac{|1-\eta|}{C_{D}} g t^{2}+2 \frac{\kappa-1}{C_{2}}\left(1-\frac{1}{\sqrt{\mu}}\right) \sqrt{\frac{|1-\eta|}{C_{D}} \frac{L_{0}}{\mu} g t} \\
& \rightarrow \frac{1-1 / \sqrt{\mu}}{1-1 / \mu} \frac{\kappa-1}{C_{2}}\left(1-\frac{1}{\sqrt{\mu}}\right) \frac{1-\eta \mid}{C_{D}} g t^{2}
\end{aligned}
$$

if $\mathrm{L}(\mathrm{t})$ varies discontinuously, resulting in the similarity parameter

$$
\frac{L(t)}{h(t)} \rightarrow \frac{\kappa-1}{C_{2}}\left(1-\frac{1}{\mu}\right),
$$

and by

$$
h(t)=\frac{1}{2} \frac{\kappa-1}{C_{2}}\left(1-\frac{1}{\sqrt{\mu}}\right) \frac{|1-\eta|}{C_{D}} g t^{2}
$$

if $\mathrm{L}(\mathrm{t})$ varies continuously, in which case we have

$$
\frac{L(t)}{h(t)} \rightarrow 2 \frac{\kappa-1}{C_{2}}\left(1-\frac{1}{\sqrt{\mu}}\right) .
$$

In conclusion, we have used a very simple model of bubble merger to illustrate and motivate the general properties of late-time multimode RT instability evolution. Specifically, we have shown how the initial conditions are erased from both the transverse and parallel scales after times much longer than the first generation of bubble merger. This forces the system into a scale invariant regime in which the wavelength grows in proportion with the perturbation amplitude, and is based on the fact that smaller bubbles merge faster than larger bubbles. Loss of initial condition information is equivalent to the relative loss of importance of increasingly higher order terms in a converging series expansion. In addition, the model suggests why $\alpha(\mathrm{A})$ of the bubble might depend weakly, if at all, on the Atwood number. 


\section{Blast-wave-driven interface motion}

The term blast wave is generally used to describe the fluid flow resulting from a strong explosion in a compressible medium, and the relevant scale-invariant solution to the Euler equations is discussed in the original works of Sedov ${ }^{44,45}$ and Taylor $^{46}$ and in several other excellent references (see, for example, Landau and Lifshitz, ${ }^{47}$ Zel'dovich and Raizer,, and Barenblatt $\left.{ }^{48}\right)$. Other than the most general overview of blast waves, it is important for our purposes to demonstrate RT instability due to the transmission of a blast wave through an interface, to determine the driving deceleration felt by the interface, and consider the effect of the large-scale fluid gradients on the developing instability. With this information in hand, we will extend the ideas of buoyancy-drag and statistical-mechanical merger models to the blast-wave-driven case.

A blast wave results when a large amount of energy is released suddenly in a small volume within a compressible medium, as in an explosion. The expanding source acts as a piston, driving a shock wave into the surrounding material. Because of the impulsive nature of the drive, the shock strength decays as the front moves away from the center. As long as the pre-shock pressure can be neglected relative to the post-shock value (ie while the Mach number $\mathrm{M}<<1$ ), the blast wave is described by a self-similar solution to the compressible Euler equations. In this idealized case, the only dimensional parameters in the problem are the energy released (E) and the 
pre-shock density $\left(\rho_{0}\right)$ in units of mass per (length) ${ }^{d}$ where $d$ is the dimension of the blast wave. Blast waves can be spherical $(d=3)$ as in the case of supernovae or other unconfined explosions, cylindrical $(\mathrm{d}=2)$, or planar $(\mathrm{d}=1)$. The term "planar blast wave" is often used to describe the flow in impulsively-driven shock tubes, including the millimeter-scale laser-driven tubes used for laboratory astrophysics experiments designed to study compressible mixing in supernovae. ${ }^{16-24}$ The motion of a gas under the action of an impulsive load is similar to, but distinct from, the solution for a planar blast wave. Whereas the position of the planar blast front scales like $\mathrm{t}^{2 / 3}$ independent of material compressibility (see below), the result in the impulsive load problem is $\mathrm{t}^{\alpha}$ where $\alpha=0.5$ at $\gamma=1$ and approaches $2 / 3$ as $\gamma$ tends to infinity. ${ }^{9}$ These differences can be approximately accounted for by applying the generalized model discussed below, which allows for arbitrary acceleration profile and velocity gradient.

The d-dimensional "radius" of the shock front must then be proportional to $\left(\mathrm{Et}^{2} / \rho_{0}\right)^{1 /(\mathrm{d}+2)}$, the only length scale that can be formed from these parameters and time. We define the constant of proportionality $\xi_{0}$ so that the shock displacement $r_{s}$ is given by

$$
r_{s}=\xi_{0}\left(\frac{E t^{2}}{\rho_{0}}\right)^{\frac{1}{d+2}}
$$

This expression is differentiated to determine the shock's velocity and deceleration as

$$
\begin{aligned}
& v_{s}=\frac{2}{d+2} \xi_{0}\left(\frac{E t^{2}}{\rho_{0}}\right)^{\frac{1}{d+2}} \frac{1}{t}=\frac{2}{d+2} \frac{r_{s}}{t} \\
& g_{s}=\frac{2}{d+2} \frac{d}{d+2} \frac{r_{s}}{t^{2}}=\frac{d}{d+2} \frac{v_{s}}{t}
\end{aligned}
$$

The similarity variable 


$$
\xi=r\left(\frac{\rho_{0}}{E t^{2}}\right)^{\frac{1}{d+2}}=\xi_{0} \frac{r}{r_{s}}
$$

varies from zero at the center to $\xi_{0}$ at the shock. Except for a time-dependent scale factor, the fluid variables must depend only on the similarity variable. The scale factors are determined by the strong-shock limit of the Rankine-Hugoniot relations, which for a polytropic equation of state require that the post-shock density, pressure, and fluid velocity are given by

$$
\begin{aligned}
& \rho_{s}=\frac{\gamma+1}{\gamma-1} \rho_{0} \\
& p_{s}=\frac{2}{\gamma+1} \rho_{0} v_{s}^{2} . \\
& u_{s}=\frac{2}{\gamma+1} v_{s}
\end{aligned}
$$

When the functions

$$
\begin{aligned}
& \rho(r, t)=\rho(\xi)=\rho_{s} \tilde{\rho}(\xi) \\
& p(r, t)=\rho(\xi, t)=p_{s}(t) \tilde{p}(\xi) \\
& u(r, t)=u(\xi, t)=u_{s}(t) \tilde{u}(\xi)
\end{aligned}
$$

are inserted into the Euler equations, they reduce to a set of ordinary differential equations for the dimensionless functions $\tilde{\rho}(\xi), \tilde{p}(\xi)$, and $\tilde{u}(\xi)$. These equations can be solved numerically or analytically, and a typical solution is shown in Fig. 3(a). Because the pressure falls of monotonically behind the shock front, an interface between two fluids is RT-unstable when it transmits a blast wave from the more dense to the less dense material.

The velocity of a lagrangian fluid element behind the shock front is determined by 

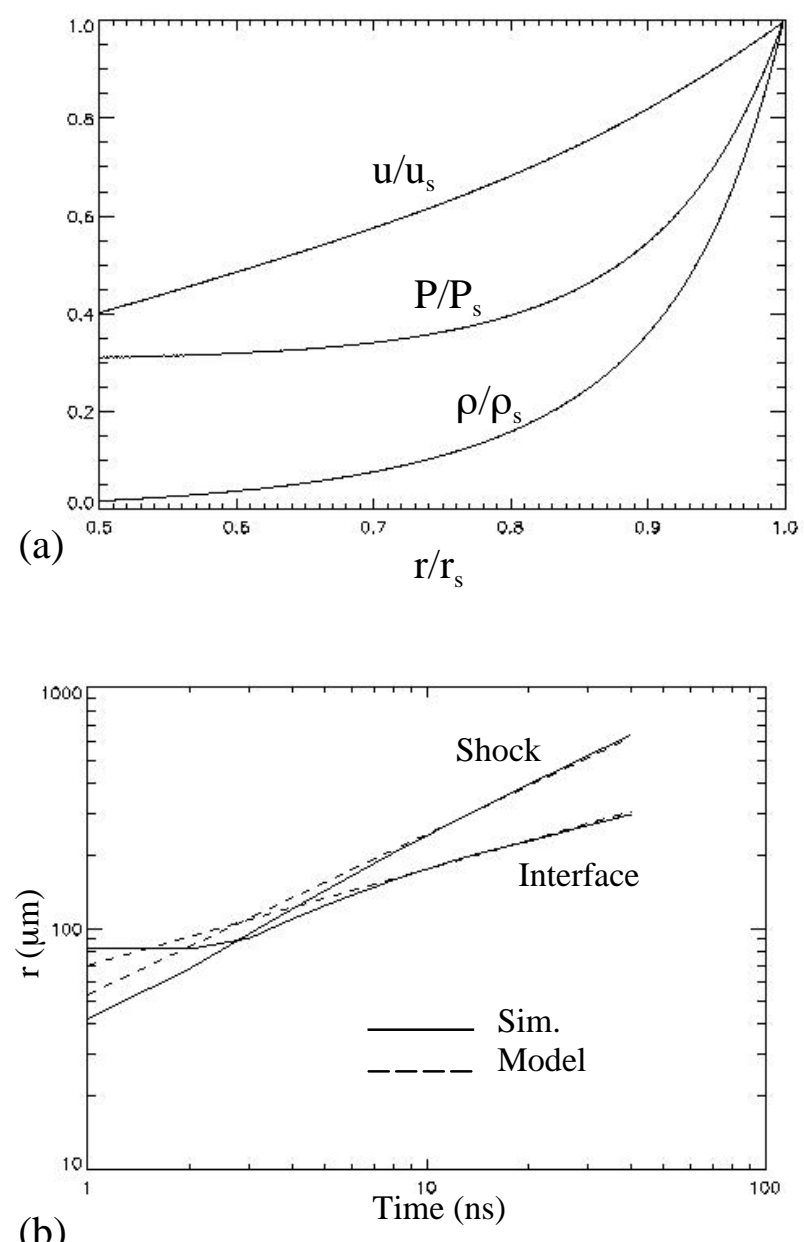

(b)

Figure 3: (a) Typical solution (with $\gamma=5 / 3$ ) to the self-similar spherical blast wave problem. (b) Motion of an interface between two identical fluids driven by a planar blast-wave. The model-predicted shock trajectory is from the self-similar solution and the interface trajectory assumes the asymptotic (linear) post-shock velocity gradient (see text). The model agrees well with the simulation after the shock has swept up twice the mass of the driver.

$$
\begin{aligned}
\frac{d u(\xi, t)}{d t} & =\frac{\partial u(\xi, t)}{\partial t}+\frac{\partial \xi}{\partial t} \frac{\partial u(\xi, t)}{\partial \xi}=\tilde{u} \frac{\partial u_{s}}{\partial t}+u_{s} \frac{d \xi}{d t} \frac{\partial \tilde{u}}{\partial \xi} \\
& =-\frac{2}{\gamma+1} g_{s}(t) \tilde{u}(\xi)\left[1+\frac{2}{d} \frac{\xi}{\tilde{u}(\xi)} \frac{\partial \tilde{u}(\xi)}{\partial \xi}\left(1-\frac{2 \xi_{0}}{\gamma+1} \frac{\tilde{u}(\xi)}{\xi}\right)\right],
\end{aligned}
$$

where the first term describes the decrease in time of the fluid velocity scale factor (the post-shock fluid velocity) and the second term describes the motion of the fluid 
element along the similarity solution. In obtaining this result, we have used the evolution equation of the similarity variable, which is given by

$$
\begin{aligned}
\frac{d \xi(r, t)}{d t} & =\frac{\partial \xi(r, t)}{\partial t}+u \frac{\partial \xi(r, t)}{\partial r} \\
& =-\frac{2}{d+2} \frac{\xi}{t}\left(1-\frac{2 \xi_{0}}{\gamma+1} \frac{\tilde{u}(\xi)}{\xi}\right)
\end{aligned}
$$

Given that the solution to the scale-invariant function $\tilde{u}(\xi)$ is known, Eq. (38) can be solved and its solution inserted into Eq. (37). This then gives the interface acceleration history $g_{i}(t)=d u\left(\xi_{i}(t), t\right) / d t$ that drives the RT instability.

The full analytic solution to the blast-wave problem with a polytropic EOS is rather cumbersome, but the asymptotic $(\xi \rightarrow 0)$ form of $\tilde{u}(\xi)$ is quite simple and given by

$$
\tilde{u}(\xi)=\frac{\gamma+1}{2 \gamma} \frac{\xi}{\xi_{0}} \Rightarrow u(r, t)=\frac{2}{d+2} \frac{1}{\gamma} \frac{r}{t} .
$$

In fact, this asymptotic solution is generally a good approximation to the full solution everywhere except just behind the shock front, where it under-predicts the velocity gradient. The boundary condition at the shock front is

$$
\tilde{u}\left(\xi_{0}\right)=1 \Rightarrow u\left(r_{s}, t\right)=\frac{2 \gamma}{\gamma+1} \frac{2}{d+2} \frac{1}{\gamma} \frac{r_{s}}{t}
$$

so the range of validity of the asymptotic solution is extended for more compressible ( $\gamma$ approaching unity) materials.

Equation (39) shows that, away from the shock front, the flow is characterized by a linear velocity gradient that decays in time, as in a centered rarefaction wave. Thus a blast wave can be thought of as a shock wave plus a rarefaction wave. 
If we insert the asymptotic solution Eq. (39) into Eq. (38) and integrate, we find that

$$
\xi_{i}(t)=\xi_{0}\left(\frac{t_{0}}{t}\right)^{\frac{2}{d+2} \frac{\gamma-1}{\gamma}}
$$

where $t_{0}=\sqrt{\rho / E}\left(r_{0} / \xi_{0}\right)^{(d+2) / 2}$ is the time at which the shock reaches the interface. The corresponding interface deceleration is then

$$
g_{i}(t)=\frac{r_{0}}{t_{0}^{2}} \frac{2}{\gamma(d+2)}\left[1-\frac{2}{\gamma(d+2)}\right]\left(\frac{t_{0}}{t}\right)^{2\left[1-\frac{1}{\gamma(d+2)}\right]},
$$

which can be integrated to obtain the interface velocity and position:

$$
\begin{aligned}
& u_{i}(t)=\frac{r_{0}}{t_{0}} \frac{2}{\gamma(d+2)}\left(\frac{t_{0}}{t}\right)^{1-\frac{2}{\gamma(d+2)}} \\
& r_{i}(t)=r_{0}\left(\frac{t}{t_{0}}\right)^{\frac{2}{\gamma(d+2)}} \cdot
\end{aligned}
$$

The position, velocity, and deceleration of the shock are recovered by setting $\gamma \rightarrow 1$ in Eqs. (42)-(44). This means that, for an infinitely compressible medium, the interface remains at the shock front at all times. As is the case of a steady shock, a more general $\gamma$ describes how quickly the shock pulls away from the interface.

Figure 3(b) shows a comparison of Eqs. (31) and (44) with a CALE ${ }^{49}$ simulation of an interface between two identical fluids driven by a planar blast wave. The simulation is initiated with a compressed slab at the origin with density and pressure appropriate for laser-driven experiments. ${ }^{24}$ When the shock reaches $200 \mu \mathrm{m}$, it has swept up twice the mass initially present in the driver. At subsequent times, the model agrees well with the simulation. 


\section{Buoyancy-drag model for blast-wave-driven case}

With expressions for the interface deceleration and velocity gradient in hand [Eq.'s (39) and (42)], we now proceed to generalize the simple buoyancy-drag and merger models presented in Section II model to the case of an interface driven by a strong blast wave. In particular, we consider how compressibility and the timedependence of the driving deceleration change the main conclusions of Section II. This extension entails three main complications.

First of all, the blast-wave-driven interface is unstable to the RichtmyerMeshkov (RM) instability ${ }^{50.51}$ in addition to RT. The RM instability results when a shock crosses a material interface, whether from light to heavy or heavy to light. The shock deposits vorticity via the baroclinic term in the vorticity equation, and the evolution of the deposited vorticity field results in perturbation growth. The simplest model of RM growth is the impulsive model, originally presented by Richtmyer, ${ }^{50}$ which treats the action of the shock as a delta-function acceleration. The impulsive model predicts that the instability grows linearly in time while the perturbation amplitude is small compared to its wavelength.

When both RM and RT are present, they do not necessarily add linearly, and there is to our knowledge no general (non-phenomenological) model that includes the effects of both. Simulations of blast-wave-driven laser experiments suggest that, for 
strong blast waves, RM dominates the instability growth very early on while RT dominates at later times. ${ }^{24}$ Single-mode simulations were well modeled by a buoyancy-drag model in which RM is approximately accounted for by simply setting the initial spike and bubble velocities equal to those predicted by the impulsive model. In what follows, we assume that the main effect of the RM component is to decrease the time required for the instability to reach the nonlinear state where the buoyancy-drag model is applicable, and make no additional accounting for RM effects.

The second complication is the time-dependence of the driving acceleration. For an interface driven by a blast wave, the deceleration is greatest just after passage of the shock front and subsequently decays in time. With a time-dependent acceleration, the number of possible length scales that can be formed in the problem independent of the perturbation scales is infinite $\left[\left(\int \mathrm{dt}^{\mathrm{n}} \mathrm{g}(\mathrm{t})^{\mathrm{n} / 2}\right)^{2 / \mathrm{n}}\right.$ for all positive integer $\mathrm{n}]$. In the classical case, these collapse into the single scale $\mathrm{gt}^{2}$. If we allow $\mathrm{g}$ in Eq. (3) to vary in time and still assume self-similarity, then we find that the mix width growth should scale like $\mathrm{s}^{34,43}$

$$
\mathrm{h} \sim\left[\int \mathrm{dt} \mathrm{g}^{1 / 2}\right]^{2}
$$

Generalizing the bubble-merger model presented for the classical case [in particular Eq. (24)], we find that the merger-time is given by

$$
(\kappa-1) \int_{t_{i}}^{t_{i}+\tau_{i}} u^{a s y m}\left(t^{\prime}\right) d t^{\prime}=(\kappa-1) C \sqrt{L_{i}} \int_{t_{i}}^{t_{i}+\tau_{i}} \sqrt{g\left(t^{\prime}\right)} d t^{\prime}=C_{2} L_{i}
$$

in the time-dependent acceleration case. Strictly speaking, $g(t)$ in Eq. (46) is the acceleration at the position of the bubble tip. In compressible systems, this is not necessarily the acceleration at the unperturbed-interface location. For the time being, 
we will nevertheless use the interface acceleration $g_{i}(t)$ as the drive for bubble growth, effectively assuming that the acceleration varies little over the bubble height. Summing both sides of (46) from $\mathrm{i}=1$ to $\mathrm{N}$ as before, we find that the transverse scale grows according to

$$
L(t)=\left[\frac{\kappa-1}{C_{2}}\left(1-\frac{1}{\sqrt{\mu}}\right) \int_{t_{0}}^{t} \sqrt{\frac{|1-\eta|}{C_{D}} g\left(t^{\prime}\right)} d t^{\prime}+\sqrt{\frac{L_{0}}{\mu}}\right]^{2}
$$

and that the late-time bubble amplitude height is given by

$$
h(t) \rightarrow \frac{\kappa-1}{C_{2}}\left(1-\frac{1}{\sqrt{\mu}}\right) \int_{t_{0}}^{t} \int_{t_{0}}^{t^{\prime}} d t^{\prime} d t^{\prime \prime} \sqrt{\frac{|1-\eta|}{C_{D}} g\left(t^{\prime}\right)} \sqrt{\frac{|1-\eta|}{C_{D}} g\left(t^{\prime \prime}\right)} .
$$

The ratio of (47) to (48) gives the same similarity parameter as in the constant $g$ case:

$$
\frac{L(t)}{h(t)} \rightarrow 2 \frac{\kappa-1}{C_{2}}\left(1-\frac{1}{\sqrt{\mu}}\right)
$$

since $\int_{t_{0}}^{t} \int_{t_{0}}^{t^{\prime}} d t^{\prime} d t^{\prime \prime} \sqrt{\frac{|1-\eta|}{C_{D}} g\left(t^{\prime}\right)} \sqrt{\frac{|1-\eta|}{C_{D}} g\left(t^{\prime \prime}\right)}=\frac{1}{2}\left[\int_{t_{0}}^{t} d t^{\prime} \sqrt{\frac{|1-\eta|}{C_{D}} g\left(t^{\prime}\right)}\right]^{2}$. Again, we recover the $\left[\int \mathrm{dt} \mathrm{g}^{1 / 2}\right]^{2}$ scaling for both $\mathrm{L}(\mathrm{t})$ and $\mathrm{h}(\mathrm{t})$ after several merger generations.

Experimental data obtained on a rocket-rig apparatus by $\operatorname{Read}^{34}$ and Dimonte et al. ${ }^{43}$ agree with this scaling for both rising and falling accelerations. This suggests that time dependence alone does not invalidate the ideas of scale invariance and loss of initial conditions.

However, it is important to note that Eq. (42) for the interface deceleration, though non-zero at all times, is only valid while the Mach number of the shock is high enough that we can neglect the pre-shock pressure $\left(M^{2}>>1 \Rightarrow M \tilde{>}\right)$. For a real blast wave, the Mach number eventually approaches unity and the interface 


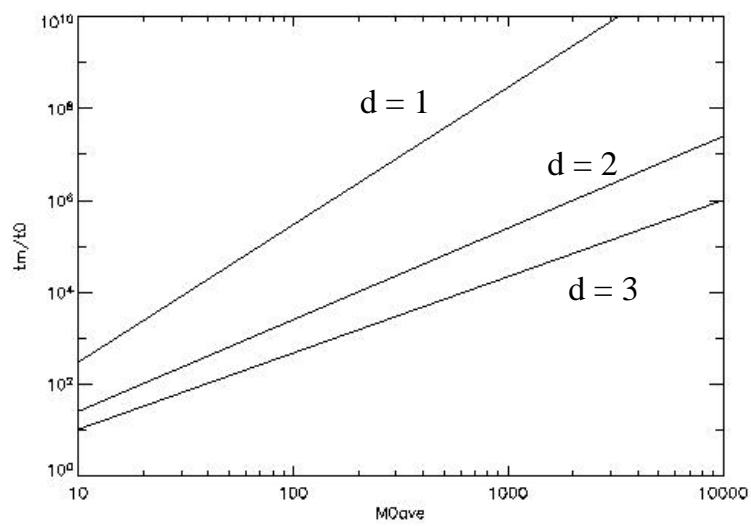

(a)

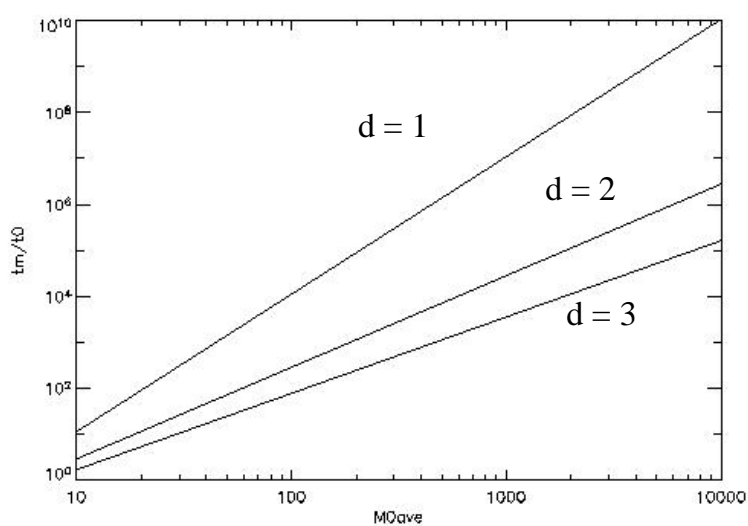

(b)

Figure 4: Estimated limiting drive time as a function of the average incident Mach number of the blast wave $\left[\mathrm{r}_{0} /\left(\mathrm{ct}_{0} /\right)\right]$. At $\mathrm{t}=\mathrm{t}_{\mathrm{m}}$, the Mach number predicted by the model in the strong shock approximation is equal to one in (a) and 3 in (b).

acceleration goes to zero. Thus the interface is driven for a finite time that we can approximate as

$$
\frac{t_{m}}{t_{0}}=\left(\frac{2}{d+2} \frac{r_{0}}{c t_{0}}\right)^{(d+2) / d}=\left(\frac{2}{d+2} M_{0}^{a v e}\right)^{(d+2) / d}
$$

or

$$
\frac{t_{m}}{t_{0}}=\left(\frac{2}{3(d+2)} \frac{r_{0}}{c t_{0}}\right)^{(d+2) / d}=\left(\frac{2}{3(d+2)} M_{0}^{a v e}\right)^{(d+2) / d}
$$

which is the time at which Eq. (32) predicts that the shock speed is equal to the preshock sound speed c [Eq. 50(a) and Fig. 4(a)] or three times the sound speed [Eq. 
50(b) and Fig. 4(b)]. In this expression, $\mathrm{M}_{0}{ }^{\text {ave }}$ is the average Mach number up to $\mathrm{t}_{0}$. $\mathrm{A}$ significant change in the shock speed due to transmission through the interface can be approximately accounted for by rescaling $\mathrm{t}_{0}$ on the right-hand side of Eq. (50). If the bubble amplitude grows to the point that it is not small compared to the interface coordinate $r_{i}$, then the drive at the bubble tip falls below the interface deceleration and the value of $\mathrm{t}_{\mathrm{m}}$ will consequently be somewhat reduced.

For blast-wave-driven systems, loss of memory of initial conditions can occur only if the time-dependent term in Eq. (47) becomes large compared to the constant term, which includes the initial transverse scale:

$$
\frac{\kappa-1}{C_{2}}\left(1-\frac{1}{\sqrt{\mu}}\right) \int_{t_{0}}^{t} \sqrt{\frac{|1-\eta|}{C_{D}} g\left(t^{\prime}\right)} d t^{\prime}>>\sqrt{\frac{L_{0}}{\mu}} .
$$

Because of the drive decay, this condition must be satisfied in a time $t<t_{m}$.

The third complication is the violation of the assumption of incompressibility. For a blast wave propagating through a single material that is initially homogeneous and isotropic, the density falls off monotonically as the material decompresses in the rarefaction behind the shock front. Because of the density gradient, spikes and bubbles experience decreasing Atwood numbers as their amplitudes increase. ${ }^{40}$ This is a relatively small effect for high Atwood number systems in the nonlinear regime, and is not accounted for in our model. A more significant effect results from the velocity gradient associated with the density gradient. In the self-similar regime (while the blast wave Mach number is large), the post-shock fluid velocity is approximately proportional to $\mathrm{r} / \mathrm{t}$ (the $\xi \rightarrow 0$ asymptotic result where $\xi$ is the similarity variable) except for just behind the shock front. Decompression provides another source of perturbation growth in addition to the RT and RM instabilities. We 
can account for the post-shock material decompression by adding the a term $\beta_{b, s} h_{b, s} / t$, where $h_{b, s}$ is the bubble (spike) amplitude, to Eq. (3) for the terminal velocity of the bubble or spike:

$$
\frac{d h_{b, s}(t)}{d t}=\sqrt{\frac{\left|1-\eta_{b, s}\right|}{C_{D}} \lambda g(t)}+\beta_{b, s} \frac{h_{b, s}}{t},
$$

where again $\eta_{\mathrm{b}, \mathrm{s}}=\rho_{\mathrm{b}, \mathrm{s}} / \rho_{\mathrm{s}, \mathrm{b}}$. The terminal velocity now depends explicitly on time and on the bubble or spike height in addition to the transverse scale and time-dependent acceleration. The coefficient $\beta$ is given in Eq. (39) as $\frac{2}{\gamma(d+2)}$ for the case of an ideal blast wave away from the shock front. Equation (52) is valid, however, for any RTunstable system in a velocity gradient proportional to $\mathrm{r} / \mathrm{t}$ and interface position that scales like $\mathrm{t}^{\beta}$. This allows for fits in systems driven by non-ideal blast waves and when $\beta$ is different in each of the spike and bubble regions. This is the only accounting we make within the model for any deviations from the single-material blast-wave solution resulting from shock transmission through the interface. Again, the situation is complicated if we account for the fact that the acceleration at the spike and bubble tips is not that same as at the unperturbed interface position. It follows from Eqs. (31), (42), and (44) that $g_{b, s}(t)=g_{i}(t)\left[1 \mp h_{b, s}(t) / r_{i}(t)\right]$, where the minus (plus) sign is for the bubble (spike). The approximation $g_{b, s}(t) \approx g_{i}(t)$, which we will always make, is valid when the perturbation amplitude is small compared to the interface radius.

Equation (52) can be integrated to yield the perturbation amplitude history for a single mode driven by a strong planar blast wave: 


$$
h(t)=h\left(t_{0}\right)\left(\frac{t}{t_{0}}\right)^{\frac{2}{\gamma(d+2)}}+2 r_{0} \sqrt{\frac{\lambda}{r_{0}} \frac{1-\eta \mid}{C_{D}}\left(\frac{\gamma(d+2)}{2}-1\right)}\left(\frac{t}{t_{0}}\right)^{\frac{1}{\gamma(d+2)}}\left[\left(\frac{t}{t_{0}}\right)^{\frac{1}{\gamma(d+2)}}-1\right] . \quad \mathrm{d}=1
$$

The effect of stretching due to material decompression is present both in the first term, resulting in stretching of the initial perturbation, and in the second term, giving enhanced stretching as the perturbation grows in amplitude. If we remove the timedependent factor in the first term and the first time-dependent factor in the second term, we recover the result for a perturbation driven by a blast-wave acceleration but without decompression. In the absence of decompression, the relative importance of the first term, which includes the initial amplitude of the perturbation, tends to zero as $\mathrm{t}$ tends to infinity. Significantly, the relative importance of the initial amplitude does not tend to zero when decompression is present. Asymptotically, the ratio of the first term in Eq. (53) to the second approaches a constant value of $\frac{h_{0}}{\sqrt{r_{0} \lambda}} \sqrt{\frac{C_{D}}{6 \gamma|1-\eta|} \frac{1}{1-\frac{2}{3 \gamma}}} \sim \frac{h_{0}}{\sqrt{r_{0} \lambda}}\left\{1, \eta^{-1 / 2}\right\}_{b, s}$, where the first factor is for bubbles and the second for spikes, for a planar blast wave. This will typically be much less than one even for bubbles, but could be significant for initially nonlinear perturbations on an interface initially located no more than a few wavelengths away from the center.

For interfaces driven by multidimensional blast waves $(d>1)$, the wavelength grows in time due to divergence according to

$$
\lambda(t)=2 \pi r_{i}(t) / m=\lambda_{0} r_{i}(t) / r_{0},
$$

where $m=2 \pi r_{0} / \lambda_{0}$ is the perturbation mode number, and Eq. (53) is no longer valid. Instead, using Eq. (54) in Eq. (52), we find 
$h(t)=h\left(t_{0}\right)\left(\frac{t}{t_{0}}\right)^{\frac{2}{\gamma(d+2)}}+\frac{2 r_{0}}{\gamma(d+2)} \sqrt{\frac{\lambda_{0}}{r_{0}} \frac{1-\eta}{C_{D}}\left(\frac{\gamma(d+2)}{2}-1\right)}\left(\frac{t}{t_{0}}\right)^{\frac{2}{\gamma(d+2)}} \ln \left(\frac{t}{t_{0}}\right) . \quad \mathrm{d}>1$

Unlike the planar case, the relative contribution of the initial amplitude in diverging systems tends to zero as late times (though only logarithmically). The continually increasing wavelength gives a continually increasing growth rate for a given acceleration, but the acceleration in higher dimensions falls off much faster than in 1D. The net result is that at late times the instability grows faster in $1 \mathrm{D}$ than in higher dimensions.

Rather than restricting ourselves to ideal blast waves, we also consider the more general case where we only require that the instability develop in a linear velocity gradient given by $\Delta \mathrm{u}=\beta \Delta \mathrm{r} / \mathrm{t}$, and that the interface trajectory follow

$$
r_{i}(t)=r_{0}\left(\frac{t}{t_{0}}\right)^{\beta}
$$

The interface is driven by an arbitrary acceleration $\mathrm{g}(\mathrm{t})$, and the evolution of a single mode is given by

$$
h(t)=h\left(t_{0}\right)\left(\frac{t}{t_{0}}\right)^{\beta}+r_{0} \sqrt{\frac{|1-\eta|}{C_{D}}}\left(\frac{t}{t_{0}}\right)^{\beta} \int_{t_{0}}^{t}\left(\frac{t^{\prime}}{t_{0}}\right)^{-\beta} \sqrt{\frac{\lambda\left(t^{\prime}\right)}{r_{0}} \frac{g\left(t^{\prime}\right) t_{0}^{2}}{r_{0}}} \frac{d t^{\prime}}{t_{0}},
$$

where the wavelength is time-dependence only in diverging systems $(\mathrm{d}>1)$.

This more general version of the model can be applied to laser-driven RT experiments in which $\mathrm{a} \sim 1 \mathrm{~ns}$ pulse from a high-powered laser is used to drive a decaying shock into a millimeter-scale beryllium shock tube. Though it resembles (and is often referred to as) a planar blast wave, the drive deviates somewhat from the self-similar Taylor-Sedov solution discussed above. Figure 5 shows a comparison of 


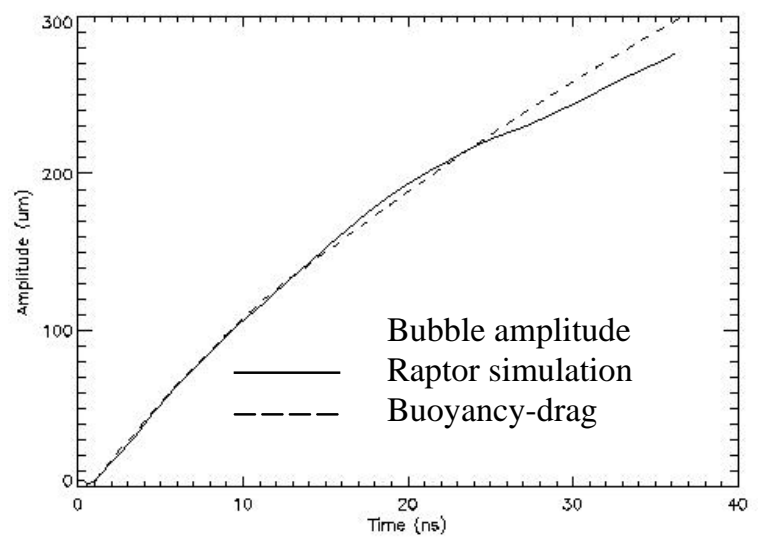

Figure 5: Application of generalized buoyancy-drag model (Eq. 57) to bubble amplitude growth in a simulation of a planar RT experiment in which a high-powered laser drives a plastic-foam interface with a pre-imposed single-mode perturbation. The drive resembles a1D blast wave with $\mathrm{M}_{0}{ }^{\text {ave }} \approx 60$. The model curve (dashed line) with $\beta=0.51$ agrees very well with the simulation (solid line).

Eq. (57) with an $A=0.7$ Raptor $^{52}$ simulation of a proposed experiment in which a 1 ns $25 \mathrm{~kJ}$ drive is applied to a single mode perturbation with $50 \mu \mathrm{m}$ wavelength and $2.5 \mu \mathrm{m}$ initial amplitude (The simulations and related experiments are discussed in detail elsewhere ${ }^{16-24,53}$ ). The model curve (dashed line) with $\beta=0.51$ agrees very well with the bubble amplitude history predicted by the simulation (solid line). A value for $\beta$ can be obtained from the simulation either by measuring the velocity gradient, which gives $\beta \approx 0.61$ or plotting the interface trajectory [using Eq. (56)], which gives $\beta \approx 0.42$. The average of these two gives the best agreement between model and simulation. Because the model assumes that spikes and bubbles instantaneously reach their terminal velocities, it does not accurately describe the spike growth at high Atwood number. In such cases, one would have to numerically solve the buoyancydrag differential equation [Eq. (2a)] for the spike velocity relative to the flow at the spike-tip position, adding at each time step the decompression velocity $\beta \mathrm{h}_{\mathrm{s}}(\mathrm{t}) / \mathrm{t}$ to find the velocity relative to the unperturbed interface. This is not necessary, however, in 
order to qualitatively capture the bubble-front evolution and associated inverse cascade to larger transverse scales.

\section{Merger model for blast-wave-driven case}

We now consider how the merger model presented in Section II is altered in the blast-wave-driven case. Rather than assume that the basic tenets of the model are the same regardless of the relative sizes of the merging bubbles, we again treat the more general case where a bubble of diameter L mergers with a larger bubble with diameter $\kappa^{2} \mathrm{~L}(\kappa>1)$. For a merger event that begins at time $t_{\mathrm{i}}$, the difference in height between the two bubbles reaches a value of $C_{2} L$ at time $t_{i+1}=t_{i}+\tau_{i}$, where $\tau$ is the merger time. Equation (57) for motion of a single bubble is applied to two bubbles of diameter $\mathrm{L}$ and $\kappa^{2} \mathrm{~L}$, and the merger time is determined from their difference:

$$
\begin{aligned}
& C_{2} \sqrt{L_{i}}\left\{\left(\frac{t_{i+1}}{t_{0}}\right)^{-\beta},\left(\frac{t_{i}}{t_{0}}\right)^{-\beta / 2}\right\}_{d=1, d>1}=\frac{\Delta h_{i}}{\sqrt{L_{i}}}\left\{\left(\frac{t_{i}}{t_{0}}\right)^{-\beta},\left(\frac{t_{i}}{t_{0}}\right)^{-\beta / 2}\right\}+ \\
& \sigma_{0}^{-1} \sqrt{r_{0}} \int_{t_{i}}^{t_{i+1}}\left(\frac{t}{t_{0}}\right)^{-\beta} \sqrt{\frac{t_{0}^{2} g(t)}{r_{0}}}\left\{1,\left(\frac{t}{t_{0}}\right)^{-\beta / 2}\right\} \frac{d t}{t_{0}}
\end{aligned}
$$

In (58), we have defined the parameter $\sigma_{0}^{-1} \equiv(\kappa-1) \sqrt{|1-\eta| / C_{D}}$ and $\Delta \mathrm{h}_{\mathrm{i}}=\mathrm{h}_{\mathrm{KL}}\left(\mathrm{t}_{\mathrm{i}}\right)$ $h_{L}\left(t_{i}\right)$ is the separation between the two bubbles at $t_{i}$. The distinction between the $d=1$ and $d>1$ is important because, in the divergent case $(d>1)$, we require that the difference in height between the two bubbles reach $\mathrm{C}_{2} \mathrm{~L}\left(\mathrm{t}_{\mathrm{i}+1}\right)=\mathrm{C}_{2} \mathrm{~L}\left(\mathrm{t}_{\mathrm{i}}\right)\left(t_{i+1} / t_{i}\right)^{\beta}$ before merger. 
Recalling that $\mu \equiv 1+\kappa^{2}$ for $2 \mathrm{D}$ perturbations and $\mu \equiv \sqrt{1+\kappa^{4}}$ for $3 \mathrm{D}$ perturbations, we find that

$$
L_{i}=\left\{\mu^{i} L_{0}, \frac{2 \pi r_{i}\left(t_{i}\right)}{m_{i}}\right\}_{d=1, d>1}=\left\{\mu^{i} L_{0}, 2 \pi r_{0}\left(t_{i}\right)\left(\frac{t_{i}}{t_{0}}\right)^{\beta} \frac{\mu^{i}}{m_{0}}\right\}=\mu^{i} L_{0}\left\{1,\left(\frac{t_{i}}{t_{0}}\right)^{\beta}\right\} .
$$

Inserting (59) into (58), we find

$$
\begin{aligned}
\mu^{i / 2} \sqrt{L_{0}}\left\{\left(\frac{t_{i+1}}{t_{0}}\right)^{-\beta}, 1\right\}_{d=1, d>1}= & \frac{\Delta h_{i}}{C_{2} \mu^{i / 2} \sqrt{L_{0}}}\left\{\left(\frac{t_{i}}{t_{0}}\right)^{-\beta},\left(\frac{t_{i}}{t_{0}}\right)^{\beta / 2}\right\}+ \\
& \frac{\sqrt{r_{0}}}{C_{2} \sigma_{0}} \int_{t_{i}}^{t_{i+1}}\left(\frac{t}{t_{0}}\right)^{-\beta} \sqrt{\frac{t_{0}^{2} g(t)}{r_{0}}}\left\{1,\left(\frac{t}{t_{0}}\right)^{-\beta / 2}\right\} \frac{d t}{t_{0}}
\end{aligned}
$$

As in the incompressible case, we wish to sum both sides in i to find an implicit relation for $\mathrm{L}_{\mathrm{N}}=\mathrm{L}\left(\mathrm{t}_{\mathrm{N}}\right)=\mathrm{L}_{0} \mu^{\mathrm{N}} \equiv \mathrm{L}(\mathrm{t})$. Carrying out the sum, we find

$$
\begin{aligned}
\left\{\sqrt{L_{0}} \sum_{i=0}^{N} \mu^{i / 2}\left(\frac{t_{i+1}}{t_{0}}\right)^{-\beta}, \frac{\sqrt{2 \pi r_{0} \mu / m_{N}}-\sqrt{L_{0}}}{\sqrt{\mu}-1}\right\}_{d=1, d>1} & =\sum_{i=0}^{N} \frac{\Delta h_{i}}{C_{2} \mu^{i / 2} \sqrt{L_{0}}}\left\{\left(\frac{t_{i}}{t_{0}}\right)^{-\beta},\left(\frac{t_{i}}{t_{0}}\right)^{\beta / 2}\right\}+ \\
& \frac{\sqrt{r_{0}}}{C_{2} \sigma_{0}} \int_{t_{0}}^{t_{N}}\left(\frac{t}{t_{0}}\right)^{-\beta} \sqrt{\frac{t_{0}^{2} g(t)}{r_{0}}}\left\{1,\left(\frac{t}{t_{0}}\right)^{-\beta / 2}\right\} \frac{d t}{t_{0}}
\end{aligned}
$$

where the perturbation mode number $m_{N}=m_{0} / \mu^{N}=2 \pi r_{0} /\left(\mu^{N} L_{0}\right)$. An important consequence follows from the fact that the sum on the left-hand side of Eq. (61) depends on the drive in the planar case but is independent of the drive in divergent systems. The second term on the right-hand side, which dominates at late times, is independent of the initial conditions. If the left-hand side reduces to a function of $\mathrm{L}(\mathrm{t})$ that is has no explicit dependence on $\mathrm{L}_{0}$ in the limit $\mathrm{L}(\mathrm{t}) / \mathrm{L}_{0}->$ infinity, then loss of memory of initial conditions is possible. For $d=1$, this is not true in general for arbitrary $g(t)$, and can only be true for special choices of the merger time such as 
$\frac{t_{i+1}}{t_{0}}=\left(\frac{L_{0} \mu^{i}}{r_{0}}\right)^{p}=\left(\frac{L_{i}}{r_{0}}\right)^{p}$ for arbitrary p. For planar blast waves, we will show that this

condition is approximately met only within a small region of the parameter space.

For diverging systems, on the other hand, we have

$$
m(t)=\left[\frac{1}{C_{2} \sigma_{0}} \frac{\sqrt{\mu}-1}{\sqrt{2 \pi \mu}} \int_{t_{0}}^{t}\left(\frac{t}{t_{0}}\right)^{-\beta / 2} \sqrt{\frac{t_{0}^{2} g(t)}{r_{0}}} \frac{d t}{t_{0}}+\sqrt{\frac{L_{0}}{2 \pi r_{0} \mu}}\right]^{-2} . \quad \mathrm{d}>1
$$

At late times, the mode number is independent of the initial conditions, as is the transverse scale

$$
L(t)=\left[\frac{\kappa-1}{C_{2} \sigma_{0}}\left(1-\frac{1}{\sqrt{\mu}}\right) \int_{t_{0}}^{t}\left(\frac{t}{t_{0}}\right)^{-\beta / 2} \sqrt{\frac{t_{0}^{2} g(t)}{r_{0}}} \frac{d t}{t_{0}}+\sqrt{\frac{L_{0}}{r_{0} \mu}}\right]^{2}\left(\frac{t}{t_{0}}\right)^{\beta} . \quad \mathrm{d}>1
$$

Inserting the time-dependent transverse scale into Eq. (57), we find that the mix width grows according to

$$
\begin{aligned}
\frac{h(t)}{r_{0}}= & \left\{\begin{array}{l}
\frac{h_{0}}{r_{0}}+\frac{1}{\sqrt{\mu}} \sqrt{\frac{L_{0}}{r_{0}}} \int_{t_{0}}^{t} d t^{\prime}\left(\frac{t^{\prime}}{t_{0}}\right)^{-\beta / 2} \sqrt{\frac{|1-\eta|}{C_{D}} \frac{t_{0}^{2} g\left(t^{\prime}\right)}{r_{0}}}+ \\
\frac{\kappa-1}{C_{2}}\left(1-\frac{1}{\sqrt{\mu}}\right) \int_{t_{0}}^{t} \int_{t_{0}}^{t^{\prime}} d t^{\prime} d t^{\prime \prime}\left(\frac{t^{\prime}}{t_{0}}\right)^{-\beta / 2} \sqrt{\frac{|1-\eta| t_{0}^{2} g\left(t^{\prime}\right)}{C_{D}}}\left(\frac{t^{\prime \prime}}{t_{0}}\right)^{-\beta / 2} \sqrt{\frac{|1-\eta|}{C_{D}} \frac{t_{0}^{2} g\left(t^{\prime \prime}\right)}{r_{0}}}
\end{array}\right\}\left(\frac{t}{t_{0}}\right)^{\beta} \\
= & \left\{\begin{array}{l}
\frac{h_{0}}{r_{0}}+\frac{1}{\sqrt{\mu}} \sqrt{\frac{L_{0}}{r_{0}}} \int_{t_{0}}^{t} d t^{\prime}\left(\frac{t^{\prime}}{t_{0}}\right)^{-\beta / 2} \sqrt{\frac{|1-\eta|}{C_{D}} \frac{t_{0}^{2} g\left(t^{\prime}\right)}{r_{0}}}+ \\
\frac{1}{2} \frac{\kappa-1}{C_{2}}\left(1-\frac{1}{\sqrt{\mu}}\right)\left[\int_{t_{0}}^{t} d t^{\prime}\left(\frac{t^{\prime}}{t_{0}}\right)^{-\beta / 2} \sqrt{\frac{|1-\eta|}{C_{D}} \frac{t_{0}^{2} g\left(t^{\prime}\right)}{r_{0}}}\right]^{2}
\end{array}\right\}\left(\frac{t}{t_{0}}\right)^{\beta}
\end{aligned}
$$

In this general form [as in Eq. (48)], the perturbative nature of the model is apparent. The last term on the right-hand side, which describes the interaction between two bubbles, is the first-order nonlinear term in a multi-bubble interaction expansion. In truncating the series after the first nonlinear term, we are assuming that interactions between three or more bubbles are insignificant compared to pair interactions. Note 
also that the initial amplitude appears only in the first term on the right-hand side and the initial transverse scale appears only in the second term on the right-hand side. The third term, which dominates at late times (as long as $\mathrm{g}(\mathrm{t})$ falls off slower than $1 / \mathrm{t}^{2}$ ) is again independent of the initial conditions, and we again find an asymptotically-selfsimilar state with the same constant ratio of transverse to parallel scales as in the incompressible case:

$$
\frac{L(t)}{h(t)} \rightarrow 2 \frac{\kappa-1}{C_{2}}\left(1-\frac{1}{\sqrt{\mu}}\right) . \quad \mathrm{d}>1
$$

In fact, Eqs. (63) and (64) are identical to Eqs. (47) and (48) for the incompressible case except for the stretching factors $\left(\mathrm{t} / \mathrm{t}_{0}\right)^{\beta}$ and $\left(\mathrm{t} / \mathrm{t}_{0}\right)^{-\beta / 2}$ in the integrals. Because of the $\left(t / t_{0}\right)^{-\beta / 2}$ factors, the time required for loss of memory of initial conditions is longer for systems in a parallel velocity gradient then it would be in incompressible systems with the same time-dependent acceleration $g(t)$.

We now summarize the specific results of Eqs. (57)-(64) applied to ideal (high Mach number) blast waves. We first treat the non-divergent (planar) case and then proceed to consider the effects of divergence on the model. With $d=1$, Eq. (58) becomes

$$
C_{2}=\frac{\Delta h_{i}}{L_{i}}\left(\frac{t_{i+1}}{t_{i}}\right)^{\frac{2}{\gamma(d+2)}}+\sigma^{-1} \sqrt{\frac{r_{0}}{L_{i}}}\left(\frac{t_{i+1}}{t_{i}}\right)^{\frac{1}{\gamma(d+2)}}\left[\left(\frac{t_{i+1}}{t_{i}}\right)^{\frac{1}{\gamma(d+2)}}-1\right], \quad \mathrm{d}=1
$$

where we have defined the parameter

$$
\sigma^{-1} \equiv 2(\kappa-1) \sqrt{\frac{\mid 1-\eta}{C_{D}}\left(\frac{\gamma(d+2)}{2}-1\right)} .
$$

Equation (66) is a quadratic in $\left(t_{i+1} / t_{i}\right)^{1 /[\gamma(d+2)]}$ that can be solved to yield 


$$
\left(\frac{t_{i+1}}{t_{i}}\right)^{\frac{1}{\gamma(d+2)}}=\frac{1}{2\left(1+\frac{\sigma \Delta h_{i}}{\sqrt{L_{i} r_{0}}}\right)}\left[1+\sqrt{1+4 \sigma C_{2} \sqrt{\frac{L}{r_{0}}}\left(1+\frac{\sigma \Delta h_{i}}{\sqrt{L_{i} r_{0}}}\right)}\right] . \quad \mathrm{d}=1
$$

If $\Delta \mathrm{h}_{\mathrm{i}}$ is zero or otherwise negligible, then we have the somewhat simpler result

$$
\left(\frac{t_{i+1}}{t_{i}}\right)^{\frac{1}{\gamma(d+2)}}=\frac{1}{2}\left[1+\sqrt{1+4 \sigma C_{2} \sqrt{\frac{L_{i}}{r_{0}}}}\right]=\frac{1}{2}\left[1+\sqrt{1+4 \sigma C_{2} \mu^{i / 2} \sqrt{\frac{L_{0}}{r_{0}}}}\right] . \quad \mathrm{d}=1
$$

Together with the Eq. (61), Eq. (68) allows us to determine in what regions of parameter space loss of memory of the initial transverse scale might be possible. The sum in (61) has the necessary properties for loss of initial conditions as long as the inequality

$$
4 \sigma C_{2} \mu^{i / 2} \sqrt{\frac{L_{0}}{r_{0}}} \ll<1 \Rightarrow \mu^{i}<<\frac{1}{4} \frac{1-\eta}{C_{D}}(\kappa-1)^{2} \frac{r_{0}}{L_{0}} \text { is satisfied. The generalization of Eq. (23) }
$$

to arbitrary $\mu$ suggests that, for classical RT, memory loss of initial conditions requires that $\mu^{N} \sim 100$, or $L=100 L_{0}$. This suggests that the above inequality is satisfied if $\frac{L_{0}}{r_{0}}<<\frac{1}{400} \frac{1-\eta}{C_{D}}(\kappa-1)^{2}$. In this case, a perturbation expansion of Eq. (68) gives $t_{i+1} / t_{i} \approx 1+\frac{\gamma(d+2) \sigma C_{2}}{\sqrt{\mu}-1}\left(\sqrt{L_{i} / r_{0}}-\sqrt{L_{0} / r_{0}}\right)$, which makes the sum in (61) independent of $\mathrm{L}_{0}$ when $\mathrm{L}_{\mathrm{N}} / \mathrm{r}_{0}$ becomes large compared to one. However, because our truncation of the perturbation expansion requires that $\mathrm{L}_{\mathrm{N}} / \mathrm{r}_{0}$ is small, we cannot conclude that memory of the initial transverse scale is lost for planar blast waves even if the initial scale is very small compared to $r_{0}$.

In general, the temporal evolution of the transverse scale can be determined by numerically solving the expression

$$
t_{N}=t_{0} \frac{t_{1}}{t_{0}} \ldots \frac{t_{N}}{t_{N-1}}=\frac{t_{0}}{2^{N}} \prod_{i=0}^{N-1}\left[1+\sqrt{1+4 \sigma C_{2} \mu^{i / 2} \sqrt{\frac{L_{0}}{r_{0}}}}\right] \quad \mathrm{d}=1
$$


for $N(t)$ and then evaluating $L(t)=L\left(t_{N}\right)=L_{0} \mu^{N(t)}$. Similarly, the evolution of the bubble height determined from

$$
h_{N}=h_{0} \frac{h_{1}}{h_{0}} \ldots \frac{h_{N}}{h_{N-1}}
$$

where, from Eq. (53), we have

$$
\frac{h_{i+1}}{h_{i}}=\left(\frac{t_{i+1}}{t_{i}}\right)^{\frac{2}{\gamma(d+2)}}\left[1+\frac{2 r_{0}}{h_{i}} \mu^{i / 2} \sqrt{\frac{L_{0}}{r_{0}} \frac{1-\eta \mid}{C_{D}}\left(\frac{\gamma(d+2)}{2}-1\right)}\left(1-\left(\frac{t_{i}}{t_{i+1}}\right)^{\frac{1}{\gamma(d+2)}}\right)\right] \cdot \mathrm{d}=1
$$

The ratio $t_{i+1} / t_{i}$ becomes large compared to unity after many generations of bubble merger, at which point

$$
\frac{h_{i+1}}{h_{i}}>\left(\frac{t_{i+1}}{t_{i}}\right)^{\frac{2}{\gamma(d+2)}} \cdot \quad \mathrm{d}=1
$$

while $L_{i+1} / L_{i}=\mu$ for all $\mathrm{i}$. Asymptotically, then, the ratio $\mathrm{L}(\mathrm{t}) / \mathrm{h}(\mathrm{t})$ (the similarity parameter) is bounded by

$$
\begin{gathered}
\frac{L_{N}}{h_{N}}=\frac{L_{N}}{L_{N-1}} \frac{h_{N-1}}{h_{N}} \frac{L_{N-1}}{h_{N-1}}<\mu\left(\frac{t_{N}}{t_{N-1}}\right)^{\frac{-2}{\gamma(d+2)}} \frac{L_{N-1}}{h_{N-1}} \\
\quad<\mu^{N}\left(\frac{t_{N}}{t_{N-1}} \ldots \frac{t_{1}}{t_{0}}\right)^{\frac{-2}{\gamma(d+2)}} \frac{L_{0}}{h_{0}}<\frac{L_{0}}{h_{0}}\left(4 \sigma C_{2} \sqrt{\frac{L_{0}}{r_{0}}} \mu^{(N-3) / 4}\right)^{-N}, \mathrm{~d}=1
\end{gathered}
$$

where we have used Eqs. (59) and (68) in addition to (72). According to (73), the similarity parameter decreases with increasing merger generation (or increasing time) at large $\mathrm{N}$. Thus there is no memory loss of initial conditions in the $\mathrm{d}=1$ case and no self-similar regime in which the mix width grows in proportion to the characteristic transverse scale. However, numerical evaluation of the model shows that, after about two generations of bubble merger, the value of the similarity parameter is nearly independent of the initial conditions (see Fig. 6). We call the quasi-self-similar 

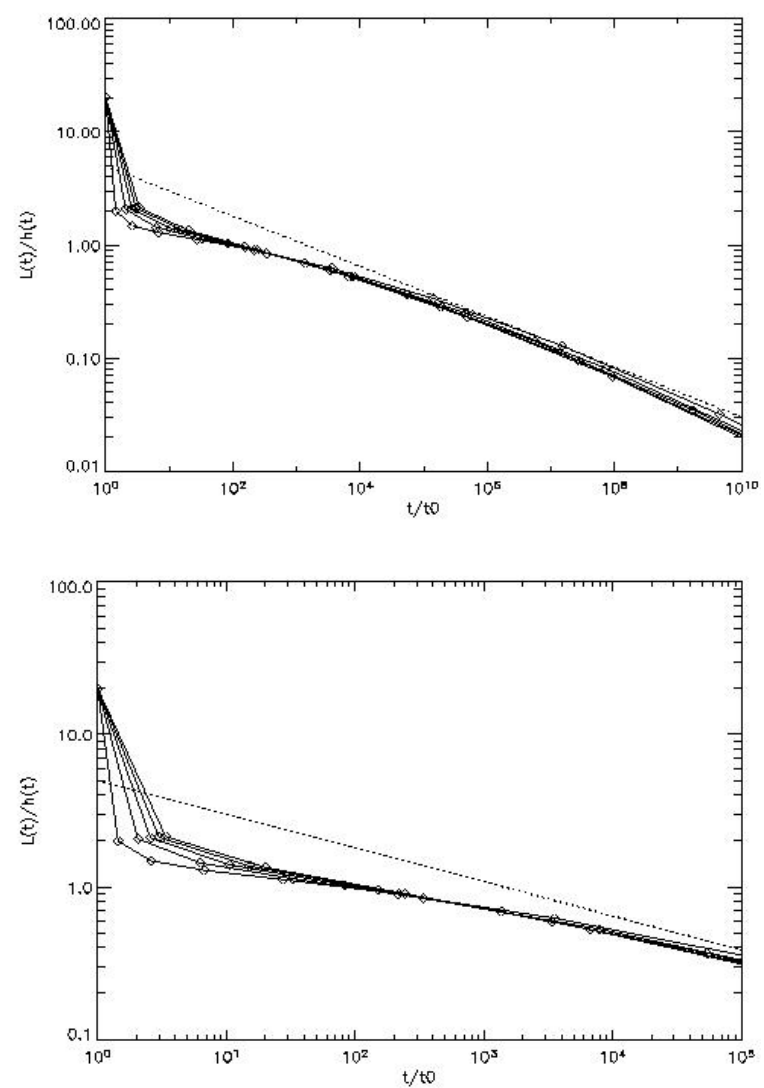

Figure 6: Quasi-self-similar regime: Rather than approaching a constant, the similarity parameter $\mathrm{h} / \mathrm{L}$ decays in time. However, the model predicts that, after about two generations of bubble merger, similarity parameter is independent of the initial conditions. In addition, the instability goes through a period of quasi-self-similar growth during which the similarity parameter changes quite slowly. At very late times, the similarity parameter scales like $\left(\mathrm{t} / \mathrm{t}_{0}\right)^{-\beta / 2}$.

regime and note that there is a period after its establishment when the similarity parameter is of order unity and changes quite slowly. At very late times, $h / L$ falls off like $\left(\mathrm{t} / \mathrm{t}_{0}\right)^{-\beta / 2}$. During this asymptotic phase, the bubble merger time has become very long and the instability evolution is dominated by amplitude stretching due to decompression.

Unless the Mach number of the incident blast wave is very high, however, it is unlikely that the $\mathrm{h} / \mathrm{L} \sim\left(\mathrm{t} / \mathrm{t}_{0}\right)^{-\beta / 2}$ state will be reached before the strong shock 
approximation is violated and the driving deceleration disappears (see Fig. 3).

Because of this limitation, there is a maximum scale, determined by the initial conditions, that can be generated at a given interface. Thus the drive imposes an "effective box size" on the system that may or may not be smaller than the actual physical or computational box size.

In the divergent case, the merger time is determined by

$$
C_{2}=\frac{\Delta h_{i}}{L_{i}}+\frac{\sigma^{-1}}{\gamma(d+2)} \sqrt{\frac{r_{0}}{L_{i}}} \ln \left(\frac{t_{i+1}}{t_{i}}\right), \quad \mathrm{d}>1
$$

from which it is easy to obtain

$$
\frac{t_{i+1}}{t_{i}}=\exp \left\{\gamma(d+2) \sigma\left(C_{2}-\frac{\Delta h_{0}}{L_{i}}\right) \sqrt{\frac{L_{i}}{r_{0}}}\right\} \rightarrow \exp \left\{\gamma(d+2) \sigma C_{2} \sqrt{\frac{L_{i}}{r_{0}}}\right\} . \mathrm{d}>1
$$

From Eq. (62) evaluated for $\mathrm{d}>1$ with the blast-wave-driven interface deceleration Eq. (42), we have

$$
m(t)=\left[\frac{1}{\sqrt{2 \pi} \gamma(d+2) C_{2} \sigma}\left(1-\frac{1}{\sqrt{\mu}}\right) \ln \left(\frac{t}{t_{0}}\right)+\frac{1}{\sqrt{\mu m_{0}}}\right]^{-2} \cdot \quad \mathrm{d}>1
$$

At late times, the mode number is independent of the initial conditions, as is the transverse scale

$$
\frac{L(t)}{r_{0}}=\left[\frac{1}{\gamma(d+2) C_{2} \sigma}\left(1-\frac{1}{\sqrt{\mu}}\right) \ln \left(\frac{t}{t_{0}}\right)+\sqrt{\frac{L_{0}}{r_{0} \mu}}\right]^{2}\left(\frac{t}{t_{0}}\right)^{\frac{2}{\gamma(d+2)}} \cdot \quad \mathrm{d}>1
$$

Inserting the time-dependent transverse scale into Eq. (57), we find that the mix width

$$
\text { grows according to } \quad d>1
$$

$$
\frac{h(t)}{r_{0}}=\left\{\frac{h_{0}}{r_{0}}+\frac{1}{\gamma(d+2) \sigma(\kappa-1)}\left[\frac{1}{2 \sqrt{2 \pi} \gamma(d+2) C_{2} \sigma}\left(1-\frac{1}{\sqrt{\mu}}\right) \ln \left(\frac{t}{t_{0}}\right)+\sqrt{\frac{L_{0}}{r_{0} \mu}}\right] \ln \left(\frac{t}{t_{0}}\right)\right\}\left(\frac{t}{t_{0}}\right)^{\frac{2}{\gamma(d+2)}}
$$


Recalling the limiting time $t_{m}=t_{0}\left(\frac{2}{d+2} M_{0}^{a v e}\right)^{(d+2) / d}$ for a blast wave, we find that there is a minimum mode number $\mathrm{m}_{\mathrm{lim}}$ that can be generated in a given divergent system, corresponding to a maximum transverse scale:

$$
m_{\lim }=2 \pi\left[\frac{1}{\gamma d C_{2} \sigma}\left(1-\frac{1}{\sqrt{\mu}}\right) \ln \left(\frac{2}{d+2} M_{0}^{a v e}\right)+\sqrt{\frac{2 \pi}{\mu m_{0}}}\right]^{-2} . \quad \mathrm{d}>1
$$

For very high Mach numbers and/or very high initial mode numbers, the limiting mode is independent of the initial mode number [see Fig. 7(a)]. Furthermore, the dependence of $\mathrm{m}_{\lim }$ on the initial Mach number (logarithmic squared) is fairly weak within the range of reasonable Mach numbers for strong blast waves [see Fig. 7(b)-7(c)]. Above about $\mathrm{M}_{0}^{\text {ave }}=20$, the $\mathrm{m}_{\text {lim }}$ varies by a factor of only a few when the Mach number varies by an order of magnitude.

Fryxell et al. have performed simulations of SN187A in which the unstable metal/He and $\mathrm{He} / \mathrm{H}$ interfaces are seeded by random grid-scale velocity perturbations behind the shock front. ${ }^{54}$ As the resolution of the calculations is varied, so too is the perturbation wavelength. At low resolution, corresponding to low initial mode number, the perturbation growth depends strongly on the resolution. At higher resolution, corresponding to a higher initial mode number of about $75-150$, the authors note that a "preferred" mode number in the range of 16-20 emerges at late times [see Fig. 8(a)]. In comparing with the merger model, we must choose an appropriate incident mach number $\mathrm{M}_{0}^{\text {ave }}$. The Mach number of the shock is actually relatively low before shock breakout into the stellar atmosphere. It is only a few in the deep interior and climbs to about $\mathrm{M} \sim 10-20$ at the $\mathrm{He} / \mathrm{H}$ interface. ${ }^{54}$ After breakout, the Mach number is of order $10^{2}$. Since this higher value determines the lifetime of 


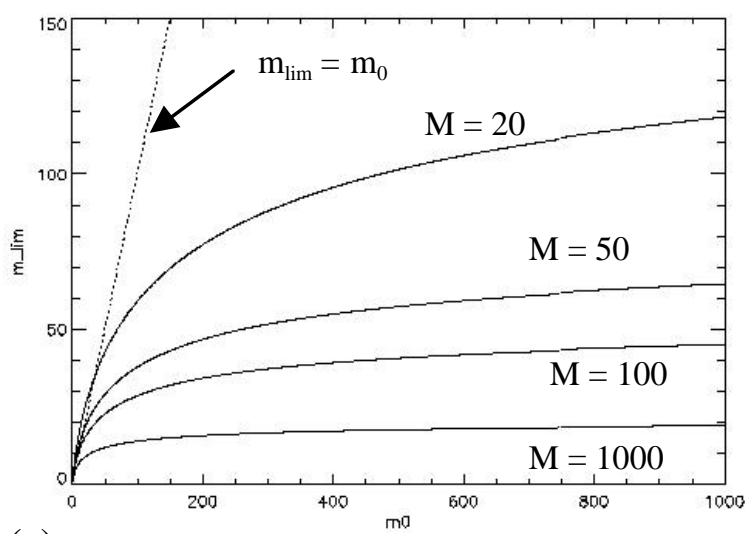

(a)

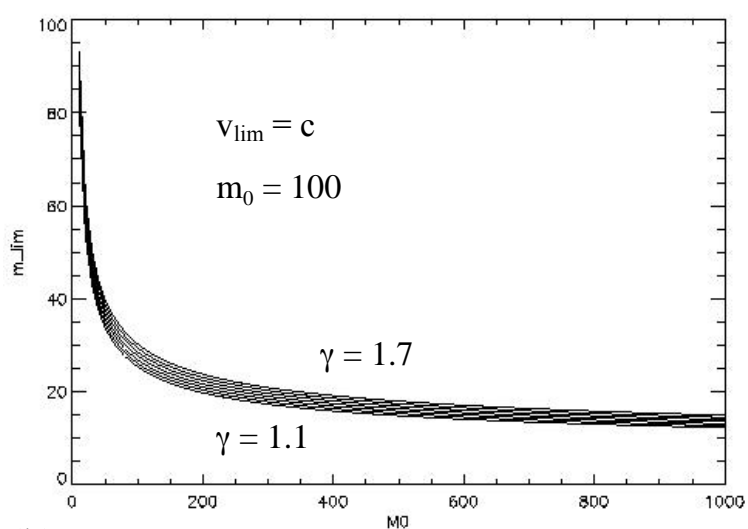

(b)

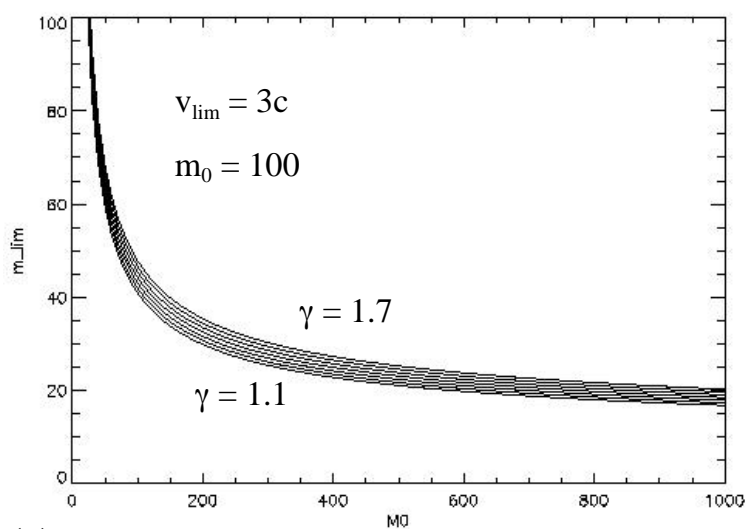

(c)

Figure 7: Minimum mode number in spherical-blast-wave-driven system. (a) For a given Mach number and high enough initial mode number, the dependence of $\mathrm{m}_{\text {lim }}$ on the initial mode number is weak. [(b)-(c)] For a given initial mode number and high enough $\mathrm{M}_{0}^{\text {ave }}$, the minimum mode number depends weakly on the adiabatic index and the incident Mach number. In (a), $\mathrm{v}_{\lim }=\mathrm{c}$ and $\gamma=1.5$. 
the blast wave, we take $\mathrm{M}_{0}{ }^{\text {ave }} \sim 100$. This suggests that memory of the initial mode number is likely to be retained until $\mathrm{t}_{\mathrm{m}}$ unless $\mathrm{m}_{0}$ is greater than about 100 [see Fig. 4(a)]. At $\mathrm{M}_{0}^{\text {ave }} \sim 100$, the model predicts a limiting (minimum) mode number $\mathrm{m}_{\lim } \sim$ 20-30, which is comparable to the preferred mode number $\mathrm{m} \sim 16-20$ found in the simulations.

We have performed a CALE $^{49}$ simulation of a hypothetical laser-driven experiment that exhibits behavior very similar to the SN calculations of Fryxell et $a l .{ }^{54}$ In the simulation [see Fig. 8(b)], we assume that a laser has been used to heat the interior of a $50 \mathrm{mg} / \mathrm{cc}, 2.5 \mathrm{~mm}$ outer diameter, $0.75 \mathrm{~mm}$-thick foam shell to $200 \mathrm{eV}$. A high Mach number spherical blast wave is driven through the shell and into the surrounding $10^{-5} \mathrm{~g} / \mathrm{cc}$ air, driving the foam/air interface RT unstable. No perturbation is pre-imposed, but a grid-generated perturbation with $\mathrm{m}_{0} \approx 100$ has appeared by $5 \mu \mathrm{s}$. After a limited merger period, a late-time mode $\approx 24$ emerges in the freeze-out stage at about $20 \mu \mathrm{s}$.

Recent simulations aimed at studying $\mathrm{RT}$ at the $(\mathrm{C}+\mathrm{O}) / \mathrm{He}$ layer within corecollapse supernovae suggest that modes as low as $\mathrm{m}_{0} \approx 24$ may be present with significant amplitudes at the pre-shock interface due to neutrino-driven convection [see Fig. 8(c)]. ${ }^{27}$ According to Eq. (79), there should likely be no significant generation of larger scales in such a system. In fact, the simulations show that the late-time interface structure is dominated by the low-m modes present in the initial conditions.

Experimental observations of late-time modal structure in RT-unstable, divergent, blast-wave-driven systems, is rather limited, and we note here only two 


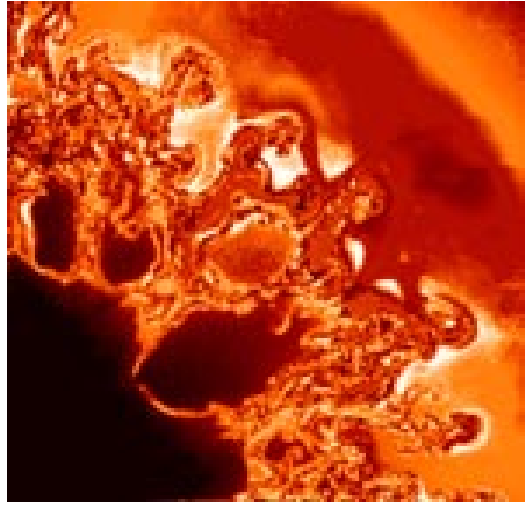

(a)
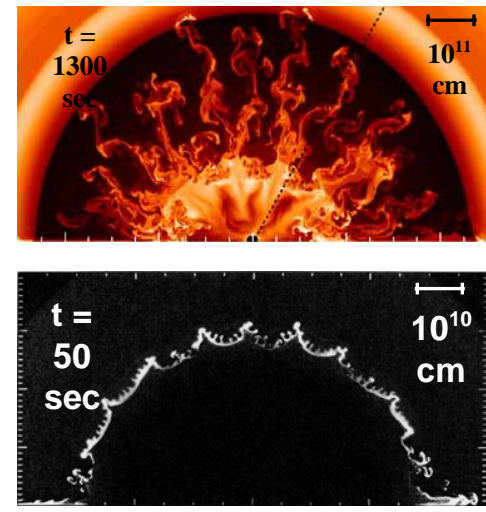

(c)
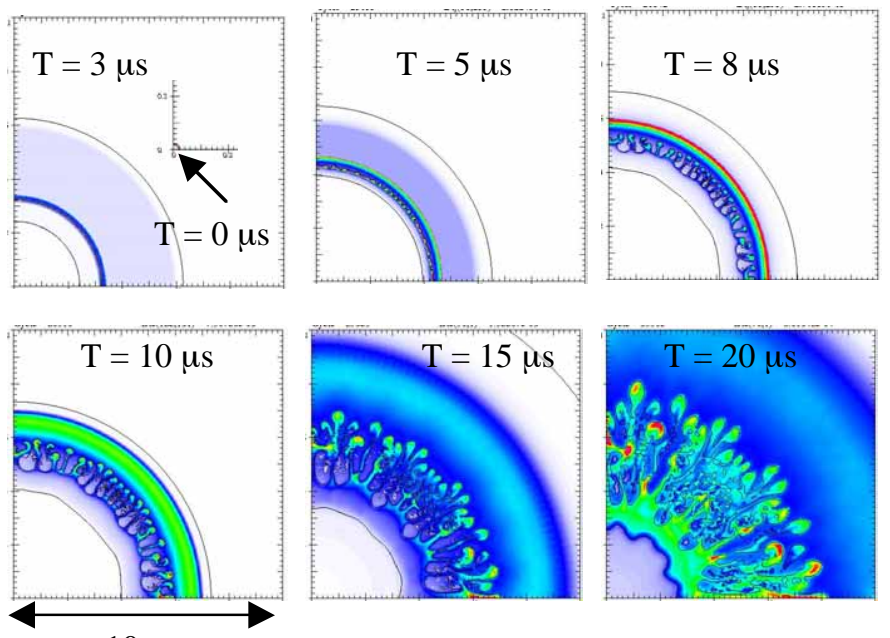

(b)

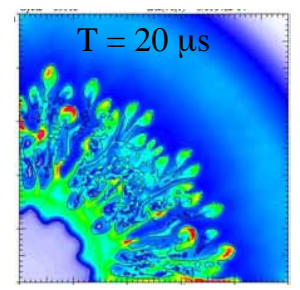

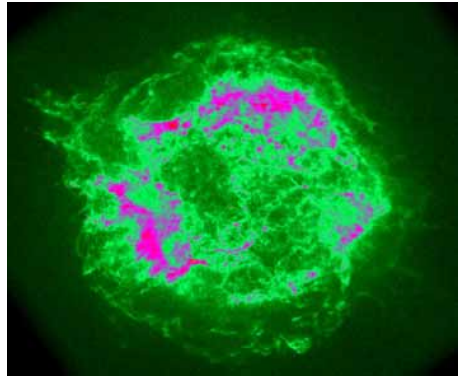

(d)

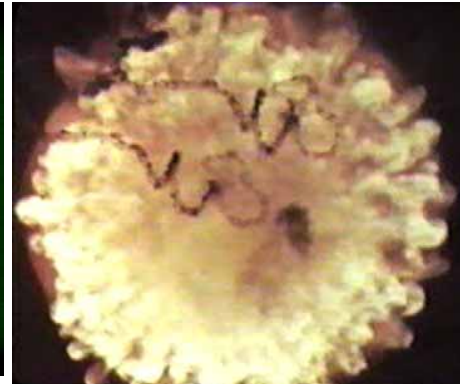

(e)

Figure 8: Similarities in asymptotic interface structure appear in a variety of systems driven by spherical blast waves. (a) Prometheus simulations of SN1987A with imposed grid-scale velocity perturbations behind the shock [Muller et al., Astron. Astrophys. 251, 505 (1991)]. When the initial mode number is high, a "preferred" mode number $\mathrm{m} \sim 16-20$ emerges at late times. (b) CALE simulation of a hypothetical laser experiment in which a spherical blast wave drives an RT-unstable foam/air interface. No perturbation is pre-imposed, but a grid-generated perturbation with $\mathrm{m}_{0} \approx 100$ has appeared by $5 \mu$ s. After a limited merger period, a late-time mode $\approx 24$ emerges in the freeze-out stage. (c) Recent SN simulations including neutrinodriven convection [Kifonidis et al., Astron. Astrophys. 408, 621 (2003)] show largescale early-time perturbations that continue to dominate at late times. (d) X-ray images of the Cassiopeia A SNR [Credit: NASA/CXC/SAO] show large-scale structures corresponding to $\mathrm{m} \sim 20$. (e) Images of high-altitude nuclear detonations [U.S. Department of Energy Nuclear Testing Archive, Las Vegas, NV] show latetime freeze-out stage with $\mathrm{m} \sim 18-36$. 
examples. First of all, x-ray images of the Cassiopeia A core-collapse supernova remnant, ${ }^{55}$ obtained via the Chandra X-Ray Observatory, ${ }^{56}$ do appear to show spikes of core material protruding out from the explosion center [see Fig. 8(d)]. The explosion does not appear to have been completely isotropic, and the spikes are not uniform in angular position or transverse scale. Nevertheless, there are several large spikes visible in one quadrant with transverse scale that corresponds to a mode number $\mathrm{m} \sim 20$, similar to that seen in both the supernova and laser experiment simulations. Similarly, film footage of high-altitude nuclear detonations shows asymptotic modal structure that suggests $\mathrm{m} \sim 18-36$ and is reminiscent of supernova simulations [see Fig. 8(e)]. ${ }^{57,58}$ In both cases, the observed minimum mode number is in the range of limiting mode numbers predicted by the model for high incident Mach number and high initial mode number. Alternatively, the late-time structure could result from low modes $(\mathrm{m}<100)$ of significant amplitude present in the initial conditions.

\section{Statistical-mechanics model for blast-wave-driven case}

The principle value of the simple two-bubble-size merger model of Section V is that, despite its simplicity, it appears to qualitatively capture several of the important aspects of multimode, nonlinear, blast-wave-driven RT. More quantitative results can be achieved by applying the same expressions for the asymptotic bubble velocity [Eq. (52)], merger time [Eqs. (58), (68), and (75)], and corresponding 


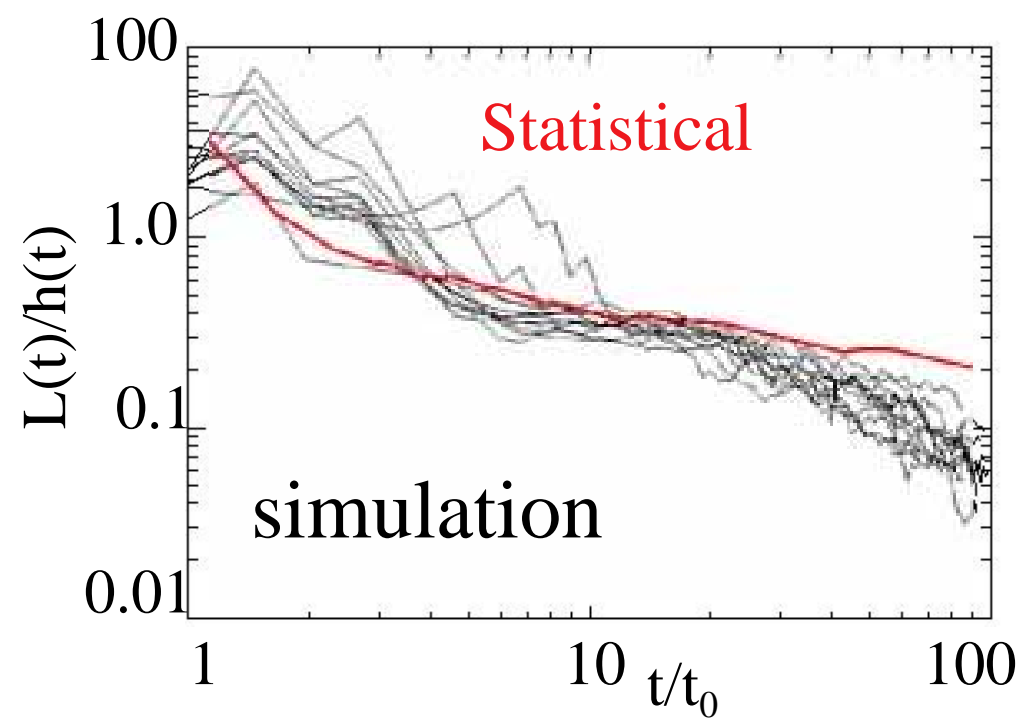

Figure 9: Time-dependence of the similarity parameter for the planar case. The prediction of the merger model applied to a broad distribution of bubble size is shown in red, and results from several 2D Raptor simulations with a variety of initial spectra are shown in black. The model-predicted value of the similarity is lower here than in the simpler two-bubble-size model of Section V (compare Fig. 6), but agrees well with the simulations.

amplitude increments [Eqs. (57), (71), and (78)], to a distribution of bubble sizes.

This allows us to follow the model-predicted evolution of the bubble-size distribution function $\mathrm{g}(\mathrm{L}, \mathrm{t})$, which is the solution to the statistical mechanical merger model equation [Eq. (4)]. Results of the statistical model represent for more sensible comparisons with experiments and simulations. In Fig. 9, we show the timedependence of the similarity parameter $\langle\mathrm{L}(\mathrm{t})\rangle / \mathrm{h}(\mathrm{t})$ for the planar case as predicted by the statistical model (shown in red), where $\langle\mathrm{L}(\mathrm{t})\rangle$ is the bubble size expectation value of $g(L, t)$ (the average bubble size), and $h(t)$ remains the extent of the mix region. For the initial conditions, bubbles are selected at random from a uniform probability distribution from zero to $0.05 \mathrm{~L}_{\mathrm{box}}$, where $\mathrm{L}_{\mathrm{box}}$ is the box size. There are then about 40 bubbles at time zero, and their initial amplitudes are all set equal to $r_{0} / 200$. Because 
the $\langle\mathrm{L}(\mathrm{t})\rangle$ contains contributions from smaller as well as dominant bubbles while $\mathrm{h}(\mathrm{t})$ is set by the largest bubbles, the similarity parameter is lower by a factor of a few than the prediction of the two-bubble-size model (compare Fig. 6). This lower value of $\langle\mathrm{L}(\mathrm{t})\rangle / \mathrm{h}(\mathrm{t})=0.2-0.4$ for $\mathrm{t} / \mathrm{t}_{0}=10-100$ is in good agreement with Raptor simulations (black curves in Fig. 9), which give $\langle\mathrm{L}(\mathrm{t})\rangle / \mathrm{h}(\mathrm{t}) \approx 0.1-0.4$ for the same $\mathrm{t} / \mathrm{t}_{0}$ and with analogous initial conditions. ${ }^{53}$ The simulations use initial spectra of various shapes (uniform, gaussian, $\mathrm{k}^{-1}$, and $\mathrm{k}^{-2}$ ) that all include modes of the same size relative to the computational box as in Fig. 9a. The drive used in the simulations is from the same planar laser-driven experiments described in Sec. IV. The agreement between the model and the simulations is particularly noteworthy in light of the fact that the interface motion in the simulations deviates somewhat from the ideal planar blast-wave-drive assumed in the model application.

\section{Conclusion}

For blast-wave-driven RT, self-similarity and loss of initial conditions are violated in the planar case but preserved in higher dimensions where divergence is present. The reason for this result is that in divergent systems the stretching factor for the transverse scales is the same as that of the parallel scales. Behind a planar blast wave, parallel scales are stretched in the rarefaction fan while transverse scales remain unaffected. Consequently, $\mathrm{L}(\mathrm{t}) / \mathrm{h}(\mathrm{t})$ decays asymptotically rather than approaching a constant value. After an early-time transient, the establishment of a 
quasi-self-similar regime is possible in systems driven by planar blast waves. Though time-dependent, the similarity parameter is nearly independent of the initial conditions during the quasi-self-similar regime. In addition, there is a period after the establishment of quasi-self-similarity but before the driving acceleration dies away during which the similarity parameter is of order unity and changes slowly in time.

The finite duration of the blast-wave drive sets a maximum scale that can be generated on a given interface. For divergent systems, this corresponds to a minimum mode number that depends weakly on the incident Mach number and initial mode number as long as both are sufficiently high. Self-similarity and loss of memory of initial conditions are in principle possible for divergent systems, but only for high initial characteristic mode numbers and high incident Mach numbers. This requirement has serious implications for supernovae. Initial conditions predicted by recent stellar calculations ${ }^{27.28}$ suggest that initial mode numbers present in supernova progenitors are not high enough to reach the self-similar regime. If these predictions are correct, the late-time interface structure observed in supernova remnants likely depends strongly on the initial conditions present within the star at the time of explosion. 


\section{Chapter 5: Effect of Initial Conditions on 2D Rayleigh-}

\section{Taylor Instability and Transition to Turbulence in Planar}

\section{Blast-wave-driven Systems}

\section{Introduction}

The post-linear evolution of the Rayleigh-Taylor (RT) instability ${ }^{1,2}$ remains incompletely understood. This is particularly true for multimode perturbations, which are also the most important for practical applications in inertial confinement fusion (ICF) and astrophysics. There is some evidence from theoretical, ${ }^{3,4}$ computational, ${ }^{5}$ and experimental ${ }^{6}$ work that memory of the initial perturbation spectrum is lost as the interface evolves into a self-similar regime in which the mix width grows in proportion with the dominant transverse scale length. The existence of such a regime has yet to be proven, however, even for the most fundamental case of incompressible fluids in a uniform gravitational field. In addition, many physical systems of interest involve compressible systems undergoing time-varying accelerations, where results obtained for the idealized case do not necessarily apply. One class of such systems includes core-collapse supernovae, in which strong blast waves propagate from near the star's core up through layers of progressively less dense material. ${ }^{7,8}$ Each driven interface is susceptible to both RT and Richtmyer-Meshkov ${ }^{9,10}$ (RM) instabilities. ${ }^{11}$ In 
addition, perturbation growth results from material expansion in the large-scale velocity gradient behind the shock front. ${ }^{12,13}$ Understanding the growth of the resulting turbulent mixing zone may be required to explain the anomalously-fast transport of core material to the star's surface..$^{7,8,14,15}$

In order to study this problem, a series of laser-driven laboratory experiments have been designed and conducted on the Nova ${ }^{16}$ and Omega ${ }^{17}$ lasers, ${ }^{12,13,18-25}$ and additional experiments are currently being planned for the National Ignition Facility ${ }^{26}$ (NIF). These experiments are intended in part to study the effect of the initial conditions on the nonlinear instability growth, the time to transition, and growth of the post-transition turbulent mixing zone for high Mach number blast-wave driven systems. In this paper, we present computational results for a planer blast-wavedriven system under NIF-like drive conditions. Using the multi-physics, AMR, higher order Godunov Eulerian hydrocode, Raptor, ${ }^{27}$ we consider the late nonlinear instability evolution for multiple amplitude and phase realizations of a variety of multimode spectral types. We show that compressibility leads to a breaking of the self-similarity and allows for memory of the initial conditions to be retained in the mix-width at all times. The loss of transverse spectral information is demonstrated, however, along with the existence of a quasi-self-similar regime over short time intervals. Aspects of the initial conditions, including the rms amplitude and characteristic wavelength, are shown to have a strong effect on the time to transition to the quasi-self-similar regime. Even different randomized amplitude and phase realizations of the same initial spectrum develop significantly different late-time amplitudes and growth rates. 


\section{Code and calculation setup}

The simulations are performed in 2D using the multi-physics radiation hydrodynamics code Raptor, which uses a $2^{\text {nd }}$ order (in space and time) Godunov method applied to the Euler equations. ${ }^{27}$ Raptor is parallelized and uses adaptive mesh refinement (AMR), making it well-suited to problems such as ours that require high resolution in only a portion of the computational domain. We use the LEOS equation of state (EOS) tables,$^{28}$ and include in the calculations electron conduction but not radiation.

Our hypothetical target [see schematic in Fig. 1(a)] represents an extension of previous and ongoing decelerating Rayleigh-Taylor experiments performed on the Omega laser and discussed in detail elsewhere. ${ }^{25}$ The cylindrical target consists of a $150 \mu \mathrm{m}$ plastic pusher section (density $1.42 \mathrm{~g} / \mathrm{cc}$ ) in contact with a less dense $2.2 \mathrm{~mm}$ payload section. An initial perturbation is machined onto the contact-surface end of the pusher. In place of the carbon foam payload used in the Omega experiments, we assume cryogenic hydrogen with density $0.086 \mathrm{~g} / \mathrm{cc}$. We expect that this change, which is motivated by uncertainties in the foam EOS tables ${ }^{13}$ would not qualitatively change the results if carbon foam was to be used in the actual experiments.

The width the computational domain was typically $200 \mu \mathrm{m}$, so that the $50 \mu \mathrm{m}$ wavelength in the previous $2 \mathrm{D}$ single-mode experiments corresponds to mode 4 . The typical resolution is 512 cells in the transverse direction (512 ppb), corresponding to 128 points per wavelength (ppw) in mode 4 . Resolution finer and coarser by up to a factor of four in each direction was used in resolution studies that are detailed below. 


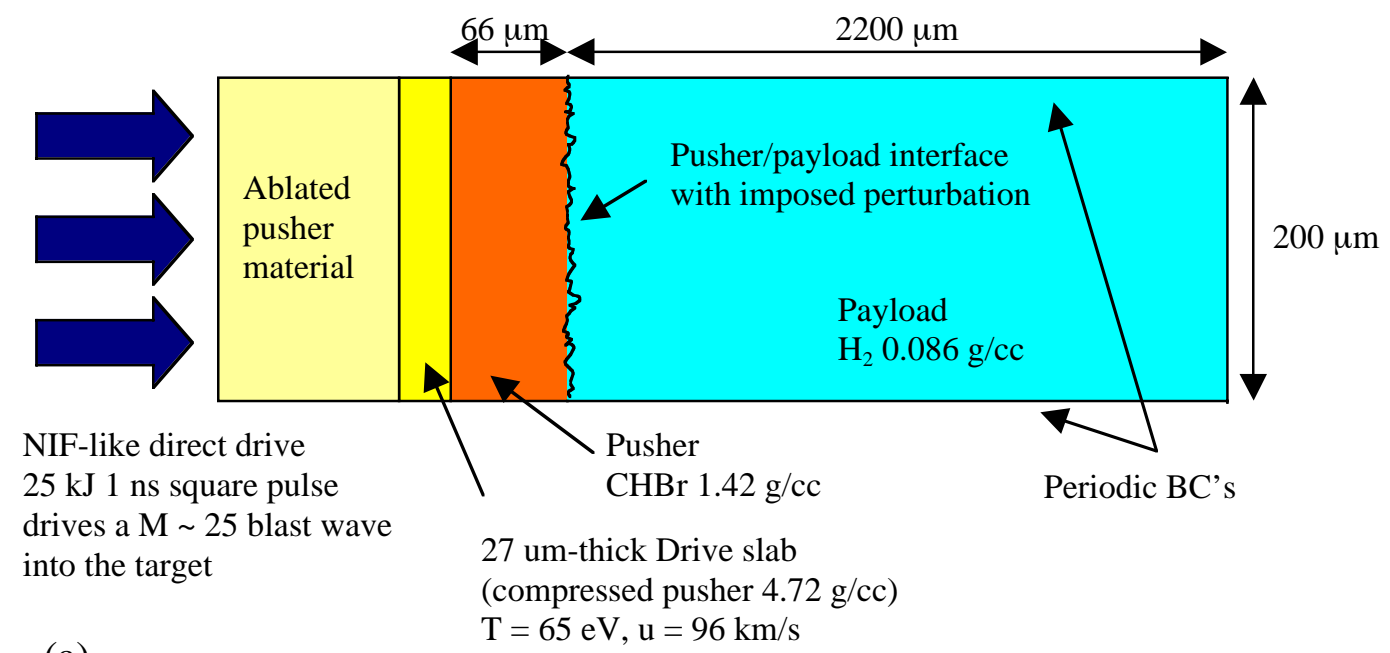

(a)

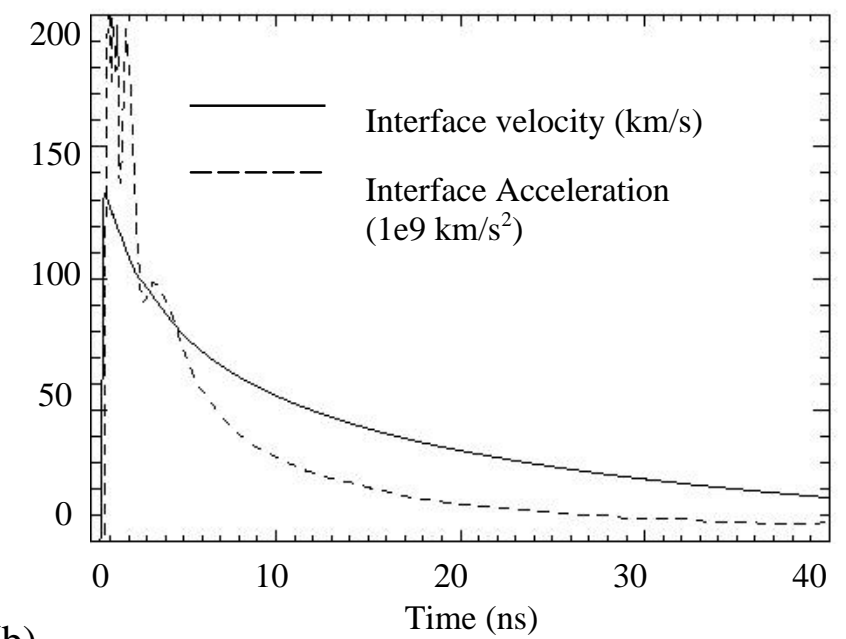

(b)

Figure 1: (a) Target schematic (not to scale). (b) Variation in time of interface velocity and deceleration.

Open boundary conditions are used in the parallel (to the shock) direction while periodic conditions are specified in the transverse direction.

The end of the pusher opposite the perturbation is driven with a $1 \mathrm{~ns}$ laser pulse, which launches a strong blast wave into the target. We assume a pulse energy of $25 \mathrm{~kJ}$ for the NIF-like drive, which is five times greater than that used in the Omega experiments. This higher laser intensity would provide significant drive over a 
longer period of time than that achieved on previous experiments, and would allow for the generation of larger transverse scales. This is important in part because bubble-merger pictures of multimode instability evolution are generally thought to require multiple merger generations above the largest significant scales present in the initial conditions before a stationary scale-invariant bubble distribution is attained. ${ }^{5} \mathrm{~A}$ systems is said to have progressed through one merger generation each time the characteristic transverse scale is doubled, so this corresponds to scales at least an order of magnitude larger than the initial conditions.

The simulations are initiated with a high-velocity, heated, compressed slab with characteristics taken from a laser-driven Lasnex $^{29}$ simulation at the end of the laser pulse.

The Mach numbers of the incident and transmitted blast waves are in the range of 10-30, where the precise value depends on the degree to which x-ray preheat can be controlled (the incident Mach number with no preheat would be about 60). The resulting initial interface speed is about $130 \mu \mathrm{m} / \mathrm{ns}$ [see Fig. 1(b)]. This is nearly twice the maximum interface speed obtained in the Omega experiments, ${ }^{13}$ and the instability is seen to develop about twice as fast. The post-shock Atwood number remains nearly constant at about 0.7 . The simulations are continued out to a maximum of $40 \mathrm{~ns}$, which is about the latest time usable data has been obtained from the Omega experiments. Throughout the duration of the experiment, the interface is RT unstable due to the reversal there of the pressure and density gradients (typical pressure, density, and velocity profiles are shown in Fig. 2). 


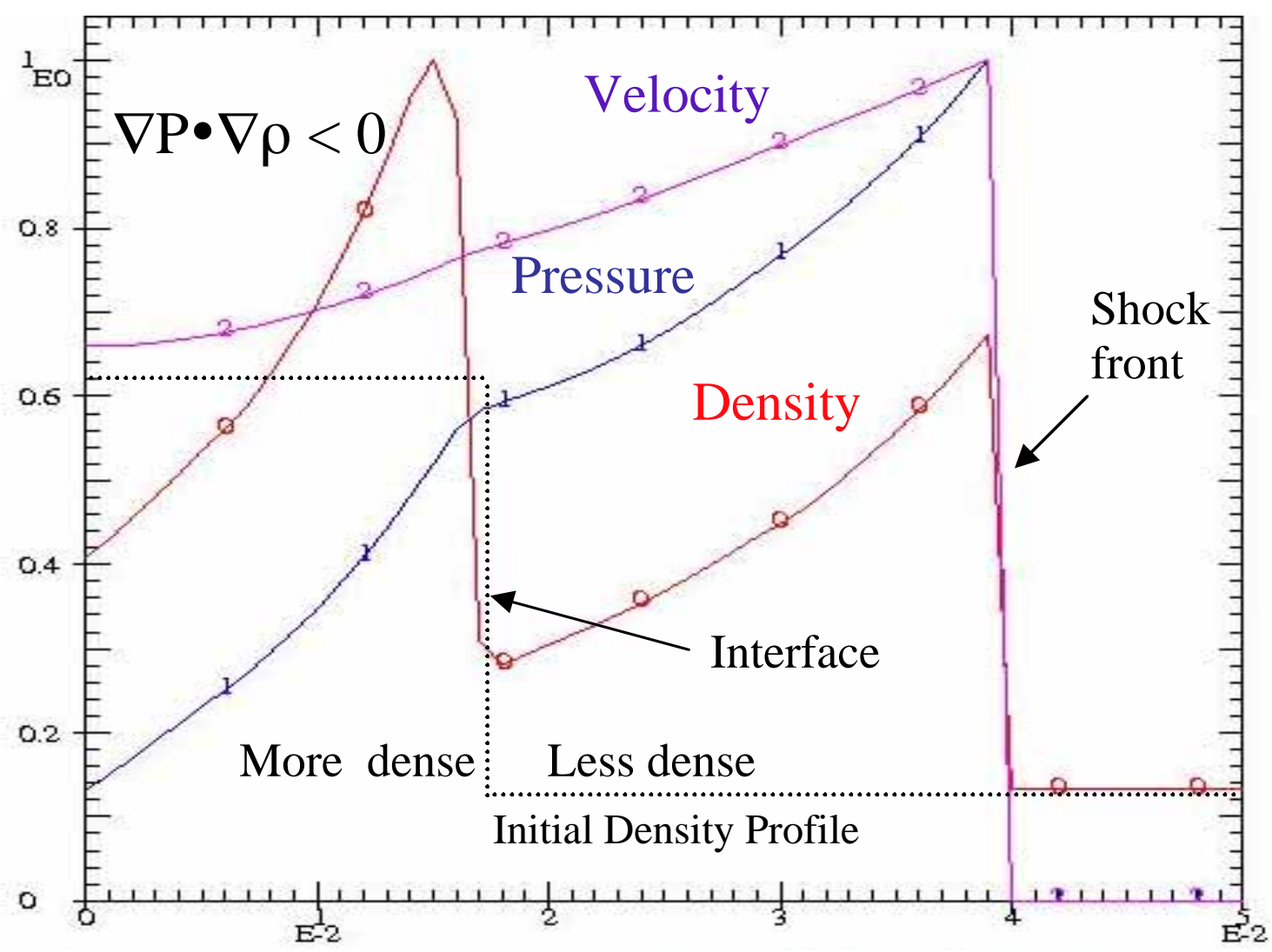

Figure 2: Pressure, density, and velocity profiles (normalized to their maximum values) after refraction of a planar blast wave through a heavy-light interface in the target. The Rayleigh-Taylor instability criterion is satisfied at the post-shock interface, which is also Richtmyer-Meshkov unstable. The fluid velocity falls off approximately linearly behind the shock front. The corresponding decompression results in additional perturbation growth.

An estimate of the relative importance of $\mathrm{RM}$ to the instability growth is obtained from "pure RM" simulations driven by a steady shock with the same strength as that of the blast wave at the time it reaches the interface. In Fig. 3, the growth rate from a pure RM calculation is compared to a blast-wave driven simulation. The same initial perturbation - large amplitude mode 4 with a narrow gaussian small amplitude short wavelength component [Fig. 4(e) and discussion below] - is used in both cases. The perturbation growth is dominated by RM for 


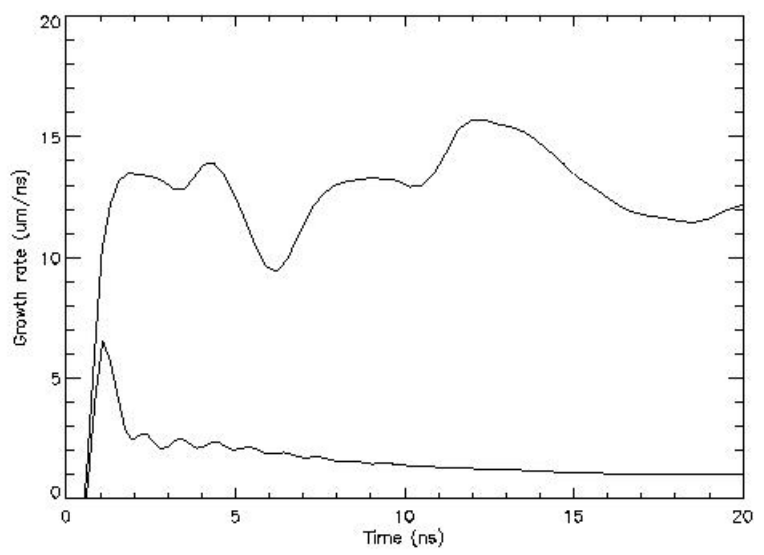

Figure 3: Comparison of pure RM and blast-wave-driven (RT+RM+decompression) growth rates suggest that RT+stretching effects dominate after about $1 \mathrm{~ns}$ of instability growth.

about the first ns, while combined RT plus decompression dominate at later times as the interface decelerates in the rarefaction behind the shock front. This is consistent with CALE simulations of Omega-driven systems in which the instability developed half as fast and RM was found to dominate for twice as long. ${ }^{13}$

\section{Characterization of initial conditions}

When we speak of dependence on initial conditions, we have in mind the effect of the initial perturbation spectrum on the observable properties of the mix region. During the linear regime when mode coupling can be neglected, this can be determined in a straightforward manner from the linear (possibly time-dependent) growth rates. Well-established mode coupling models make the weakly nonlinear regime fairly tractable as well. ${ }^{30-32}$ Our interest is the effect of the initial conditions on 

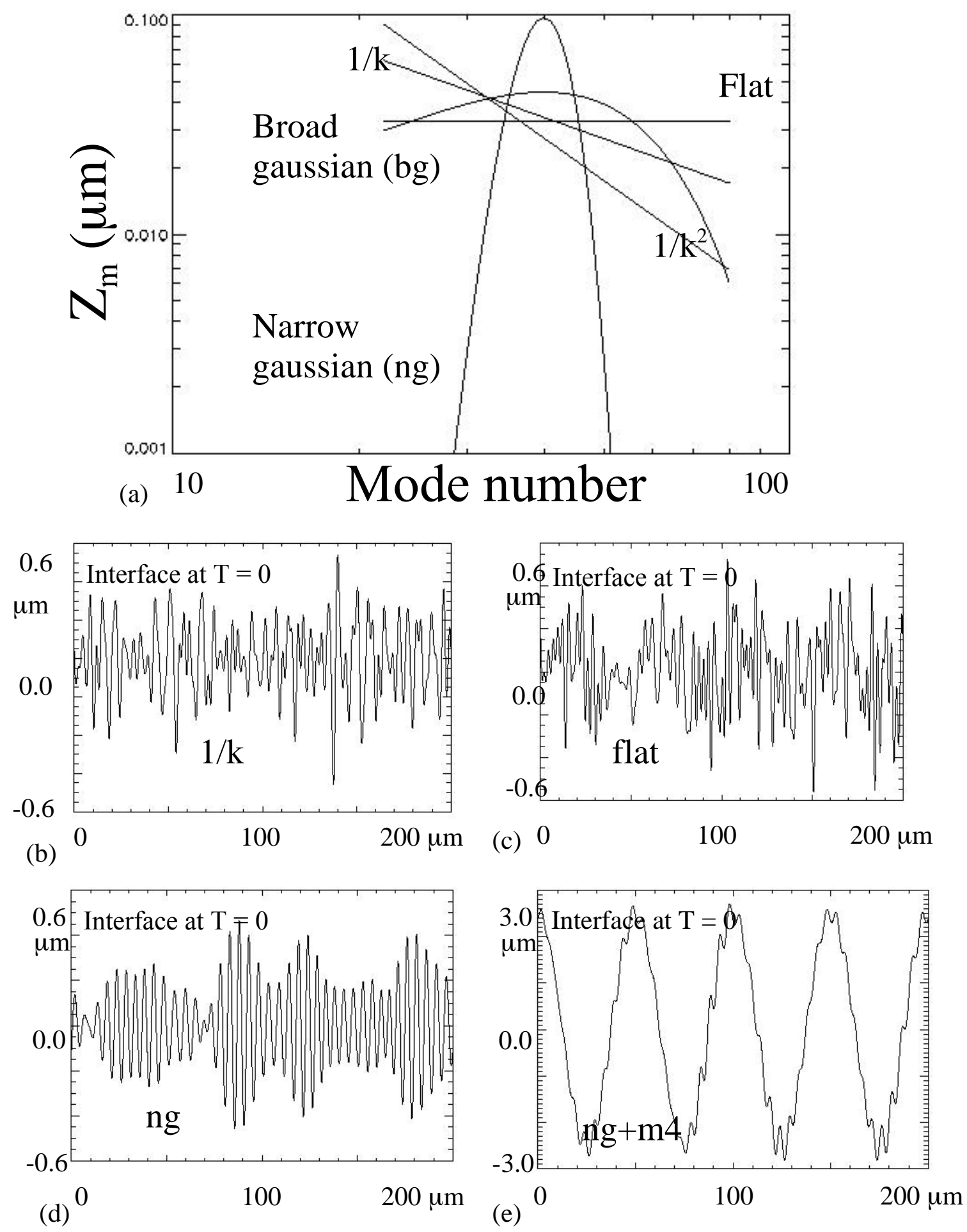

Figure 4: (a) Initial spectral shapes and typical interface profiles: (b) hyperbolic (1/k), (c) flat, (d) narrow gaussian, (e) and narrow gaussian with large-amplitude mode 4. 
the instability growth in the strongly nonlinear regime; before, during, and after any transition to a self-similar or turbulent state.

The principle observables during the deep nonlinear phase are the amplitudes and growth rates of the spike and bubble fonts, and the statistical properties of the internal structure of the mix region. The internal structure of the interface region is characterized by spectra (density, kinetic energy, velocity, and enstrophy) and by the degree of small-scale mixing of the two fluids.

The most general distinction we make in classifying initial spectra is between continuous and bi-component spectra. Bi-component spectra include a longwavelength component and a separated (in k-space) short-wavelength component. In this study, the long-wavelength component always consists of a single mode (mode 4) with a wavelength of $50 \mu \mathrm{m}$ and initial amplitude that is typically $2.5 \mu \mathrm{m}$. This is the same mode used in ongoing Omega experiments that we have previously modeled extensively. ${ }^{13}$ With its nominal initial amplitude, mode 4 is only marginally linear $(\mathrm{a} / \lambda=0.05)$. The post-shock amplitude, however, is an order of magnitude smaller. The bi-component spectral class is particularly important for considering potential effects of short-wavelength "noise" - possibly unresolved in calculations or not included in simple experiments - on the large-scale interface structure.

Five different spectral shapes were considered for the continuous class, and four of these were also used for the short wavelength component of the bi-component class. In a typical case, random phases were assigned to each mode and randomized amplitudes were selected from the given spectrum. For example, a flat spectrum 
included modes 4 to 80 with random phases and amplitudes chosen from a uniform distribution. After the amplitude assignment, the resulting spectrum is normalized to give the desired rms amplitude - typically either about $2.5 \mu \mathrm{m}$ (large amplitude case) or $0.25 \mu \mathrm{m}$ (small amplitude case). The other four spectral types include a short wavelength component either with or without a single large amplitude long wavelength mode (mode 4) in order to investigate the effect of short wavelength noise on a long wavelength primary mode. The short wavelength component, which includes modes 20 to 80 , is given by either a narrow gaussian centered at mode 40 with half-width $\approx 4$, a broad gaussian centered at mode 40 with half-width 20 , a hyperbolic $(1 / \mathrm{k})$ specrum, or a $1 / \mathrm{k}^{2}$ spectrum. Examples of initial spectra and interface profiles are shown in Fig. 4. Multiple randomized amplitude and phase realizations were generated from each spectral type in order to provide information about the typical level of fluctuations of measurable quantities within each spectrum.

We can characterize continuous spectra by their initial rms amplitude $\mathrm{a}_{\mathrm{rms}}$ (or $\langle\mathrm{a}\rangle_{0}$ ), their characteristic wavenumber $\langle\mathrm{k}\rangle$ ( or characteristic mode number $\langle\mathrm{m}\rangle$ ), the initial degree of linearity $\langle\mathrm{ka}\rangle_{0}$, the initial spectral shape, and the width of the initial spectrum $\Delta \mathrm{k} /<\mathrm{k}>$. For bi-component spectra, we can add to our parameter list the relative rms amplitudes $\left\langle a{ }_{0 s}\right|\langle a\rangle_{01}$, the relative widths of the spectral components $\Delta \mathrm{k}_{\mathrm{s}} / \Delta \mathrm{k}_{\mathrm{l}}$, and the separation of the relative two components $\left\langle\mathrm{k}_{\mathrm{s}}\right\rangle \mid\left\langle\mathrm{k}_{\mathrm{l}}\right\rangle$ or $\left(\left\langle\mathrm{k}_{\mathrm{s}}\right\rangle-\right.$ $\left.\left\langle\mathrm{k}_{1}\right\rangle\right) /\left(\left\langle\mathrm{k}_{\mathrm{s}}\right\rangle+\left\langle\mathrm{k}_{\mathrm{l}}\right\rangle\right)$. In the bi-component case, we focus in particular on the relative rms amplitudes and spectral shape. The ratio of characteristic wavelengths is only varied from $9.25\left(1 / \mathrm{k}^{2}\right.$ spectrum) to 11.30 (broad gaussian), with the two components always well-separated in k-space (by an order of magnitude). Since the width of the 
long-wavelength component is in every case a single mode, all variation of the relative spectral widths is left to the short-wavelength component. The initial spectral width can be thought of as one aspect of the spectral shape, and was only considered in this context in the bi-component case.

A list of these parameters is given in Table 1 together with a brief summary of their effect on the large-scale observables in the simulations. Not surprisingly, we found a link between the two measures used to characterize the internal structure. The simulations all exhibit a transition to a well-mixed state (a "2D mixing transition") that is correlated with a loss of transverse spectral information. Consequently, the effect of the initial conditions on the nonlinear interface structure is represented simply as their effect on the time to transition. Furthermore, transition results in changes in the spike and bubble growth rates, so anything that affects the transition time also affects the perturbation amplitudes and velocities. In the next sections, the information in Table 1 is developed in detail.

\section{$\underline{\text { IV. Results and discussion }}$}

\section{A. Growth of the mix layer}

Mix width history plots from 52 2D simulations are shown in Fig. 5. Most of the various trajectories fall in to one or the other of two families. The upper family contains the runs with the large amplitude $(2.5 \mu \mathrm{m})$ mode 4 in the initial spectrum with or without a short wavelength small amplitude component. Short wavelength components included in the plot are the narrow gaussian, broad gaussian, or 


\begin{tabular}{|c|c|c|c|}
\hline Spectral parameters & $\begin{array}{l}\text { Range of parameter } \\
\text { variation and Notes }\end{array}$ & $\begin{array}{l}\text { Effect of parameter } \\
\text { increase on: } \\
\text { Perturbation } \\
\text { amplitudes and } \\
\text { growth rates }\end{array}$ & \begin{tabular}{|l}
$\begin{array}{l}\text { Effect of } \\
\text { parameter } \\
\text { increase on: }\end{array}$ \\
Time to transition
\end{tabular} \\
\hline \multicolumn{4}{|l|}{ Continuous spectra } \\
\hline rms amplitude $\langle a>$ & $0.0025 \mu \mathrm{m}-2.5 \mu \mathrm{m}$ & Increase & Decrease \\
\hline $\begin{array}{l}\text { Characteristic } \\
\text { wavelength }\langle\mathrm{k}\rangle\end{array}$ & $\langle\mathrm{m}\rangle=4-46$ & Decrease & Decrease \\
\hline $\begin{array}{l}\text { Initial nonlinearity } \\
\text { ka> }\end{array}$ & $\begin{array}{l}\langle\mathrm{a} / \lambda\rangle=5 \mathrm{e}-4-5 \mathrm{e}-2 \\
\text { Sets thresholds for } \\
\text { spike interaction and } \\
\text { transition. }\end{array}$ & $\begin{array}{l}\text { Effect contained in } \\
\text { Individual } \\
\text { dependence on }\langle\mathrm{a}\rangle \\
\text { and }\langle\mathrm{k}\rangle\end{array}$ & Decrease \\
\hline Spectral shape & $\begin{array}{l}\text { Single mode, flat, } \\
\text { narrow gaussian, } \\
\text { broad gaussian, } \\
1 / \mathrm{k}, 1 / \mathrm{k}^{2}\end{array}$ & Little effect & Little effect \\
\hline Spectral width $\Delta \mathrm{k} /<\mathrm{k}>$ & $\begin{array}{l}0-1.8 \\
\text { Not varied independent of } \\
k \mathrm{k}>\end{array}$ & $\begin{array}{l}\text { Decrease for given } \\
\mathrm{k}_{\text {min }} .\end{array}$ & $\begin{array}{l}\text { Decrease for given } \\
k_{\text {min }}\end{array}$ \\
\hline \multicolumn{4}{|l|}{$\begin{array}{l}\text { Bi-component } \\
\text { spectra (long + shorts) }\end{array}$} \\
\hline $\begin{array}{l}\text { Shorts/longs rms } \\
\text { amplitude ratio } \\
\langle\mathrm{a}\rangle_{\mathrm{s}} \mid\langle\mathrm{a}\rangle_{1}\end{array}$ & $\begin{array}{l}(<0.001)-0.1 \\
\text { Effect on bubble growth } \\
\text { may be opposite for } \\
\text { multimode long wavelength } \\
\text { component }\end{array}$ & $\begin{array}{l}\text { Decrease for spikes } \\
\text { and increase for } \\
\text { bubbles }\end{array}$ & Decrease \\
\hline $\begin{array}{l}\text { Spectral shape of } \\
\text { shorts }\end{array}$ & $\begin{array}{l}\text { Narrow gaussian, broad } \\
\text { gaussian, } 1 / \mathrm{k}, 1 / \mathrm{k}^{2}\end{array}$ & Little effect & Little effect \\
\hline Spectral shape of longs & Not varied & & \\
\hline $\begin{array}{l}\text { Ratio of characteristic } \\
\text { wavelengths } \\
\langle\mathrm{k}\rangle_{\mathrm{s}} \mid\langle\mathrm{k}\rangle_{1}\end{array}$ & $\begin{array}{l}9.50-11.30 \\
\text { Not varied } \\
\text { independent of shape. }\end{array}$ & $\begin{array}{l}\text { Little effect over } \\
\text { range considered }\end{array}$ & $\begin{array}{l}\text { Little effect over } \\
\text { range considered }\end{array}$ \\
\hline $\begin{array}{l}\text { Ratio of spectral } \\
\text { widths } \Delta \mathrm{k}_{\mathrm{s}} / \Delta \mathrm{k}_{1}\end{array}$ & $\begin{array}{l}\text { Not varied } \\
\text { independent of } \\
\text { relative amplitude } \\
\text { and shape }\end{array}$ & Inconclusive & Inconclusive \\
\hline
\end{tabular}

Table 1: List of the parameters used to classify and characterize the initial spectral conditions and a summary of their effect on the nonlinear instability evolution. See text for detailed explanation. 

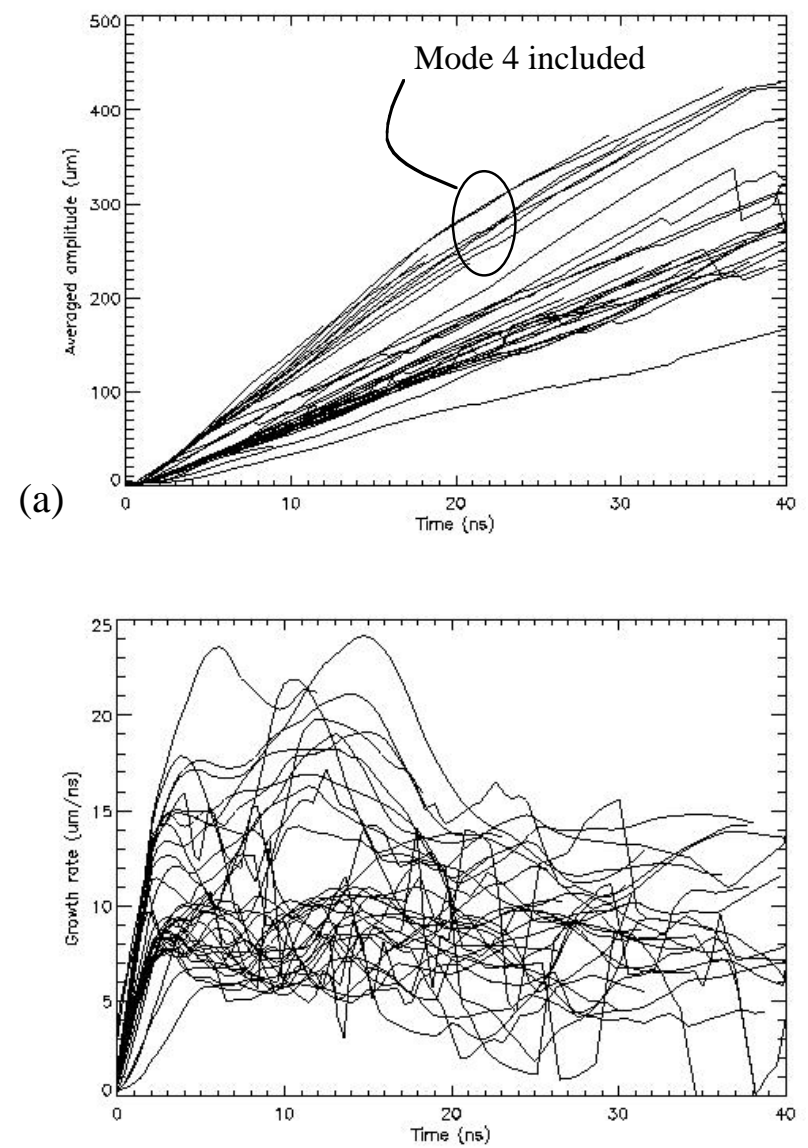

(b)

Figure 5: (a) Mix width histories and (b) growth velocities from 52 2D simulations with different spectral initial conditions. There is no apparent approach to a selfsimilar regime independent of the initial conditions. Even when runs with large mode 4 are excluded, $\alpha_{b}$ varies over a range of about $0.035-0.065$ for different IC's, while $\alpha_{\mathrm{s}}$ varies over about $0.050-0.100$.

hyperbolic spectrum, or by a single mode 40 . The rms amplitude in each case differs from the mode 4 amplitude by less than $1 \%$. The lower family consists primarily of runs with the small amplitude short wavelength component, with mean rms amplitude of about $0.25 \mu \mathrm{m}$. The standard deviation from the mean rms amplitude is less than $5 \%$, and the maximum deviation is less than $50 \%$. The small amplitude flat spectrum cases are also contained within the lower family. The large amplitude flat spectrum 
cases initially lie slightly above the upper family, but then fall below it at about 2-3 ns, eventually joining the lower family between 10 and 20 ns. The two curves below the lower family are from runs with rms amplitude of $0.025 \mu \mathrm{m}$ (upper curve) and $0.0025 \mu \mathrm{m}$ (lower curve).

Within the lower family, the amplitude is not well correlated with initial rms amplitude variations at the few-percent level. Furthermore, the difference between runs with different spectral shape (but similar initial rms amplitude) is generally not much greater than the difference seen between different amplitude and phase realizations of the same spectrum. This is illustrated in Fig. 6, which compares the bubble amplitude and velocity evolution for several spectral shapes, including two random phase realizations of the same narrow gaussian distribution without amplitude randomization (dotted lines). Solid lines show the amplitude from simulations with a 1/k spectrum (upper solid), flat (middle solid), and broad gaussian (lower solid). All five simulations begin with an rms-amplitude of $0.258 \mu \mathrm{m}$. After $10 \mathrm{~ns}$, the amplitude and velocity difference between the two narrow gaussian cases is greater than $25 \%$ and is at least as significant as the differences arising from the various spectral shapes.

\section{B. Phases of instability growth}

In general, the instability evolution can be divided into three phases, as shown in Figure 7. During the early-time phase (Phase I - which actually included the linear early nonlinear, and into the nonlinear regimes), the growth rate is determined by the most unstable mode. RM dominates initially, but only for about $1 \mathrm{~ns}$. During this 


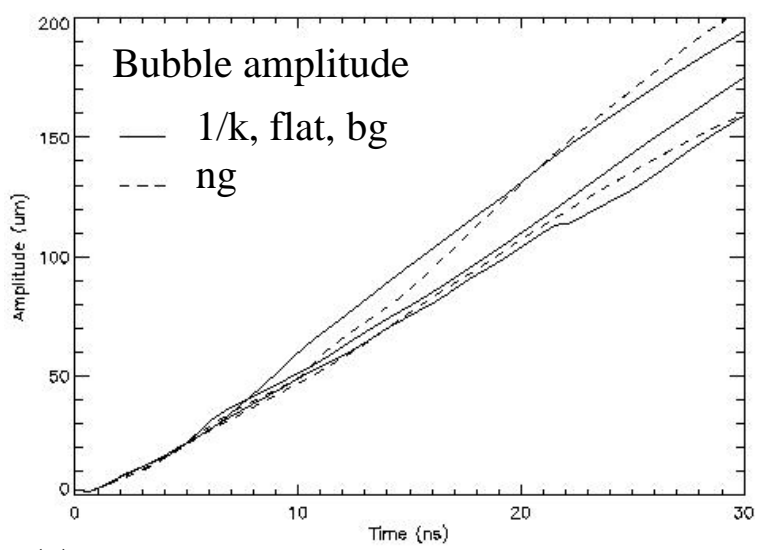

(a)

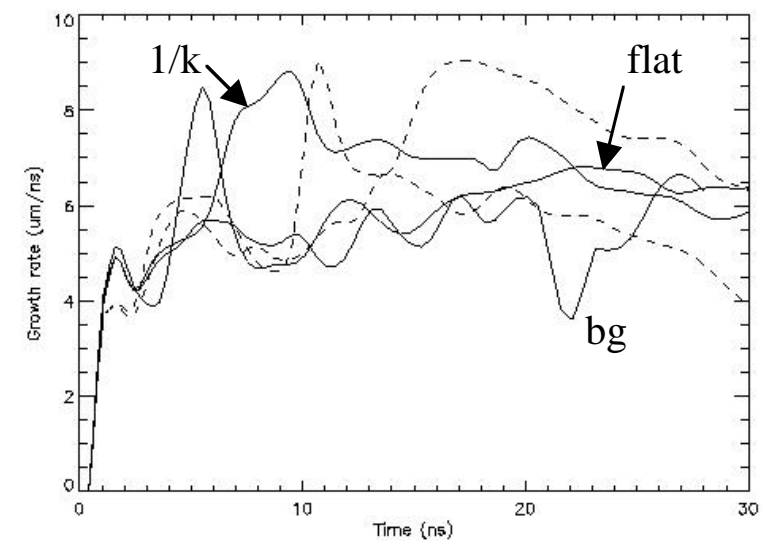

(b)

Figure 6: Sensitivity of bubble (a) amplitude and (b) velocity to spectral shape. Solid lines denote three initial spectra: 1/k [upper solid in (a)], flat [middle solid in (b)], and broad gaussian [lower solid in (a)]. The dotted lines denote two phase realizations of the same narrow gaussian spectrum. In each case, the initial rms-amplitude is 0.258 $\mu \mathrm{m}$.

period, the inverse cascade to larger scales is initiated, and there are up to three generations of bubble merger. The growth rate depends on the rms amplitude, but does not depend strongly on the spectral details. During Phase II, there are changes in the growth rates (sometimes rather abrupt) that result in a strong dependence on the spectral details as well as the initial rms amplitude. Consideration of separate spike and bubble amplitude histories shows that the spike growth is more sensitive than the bubble growth to the initial spectrum. These changes appear to be random and are not 

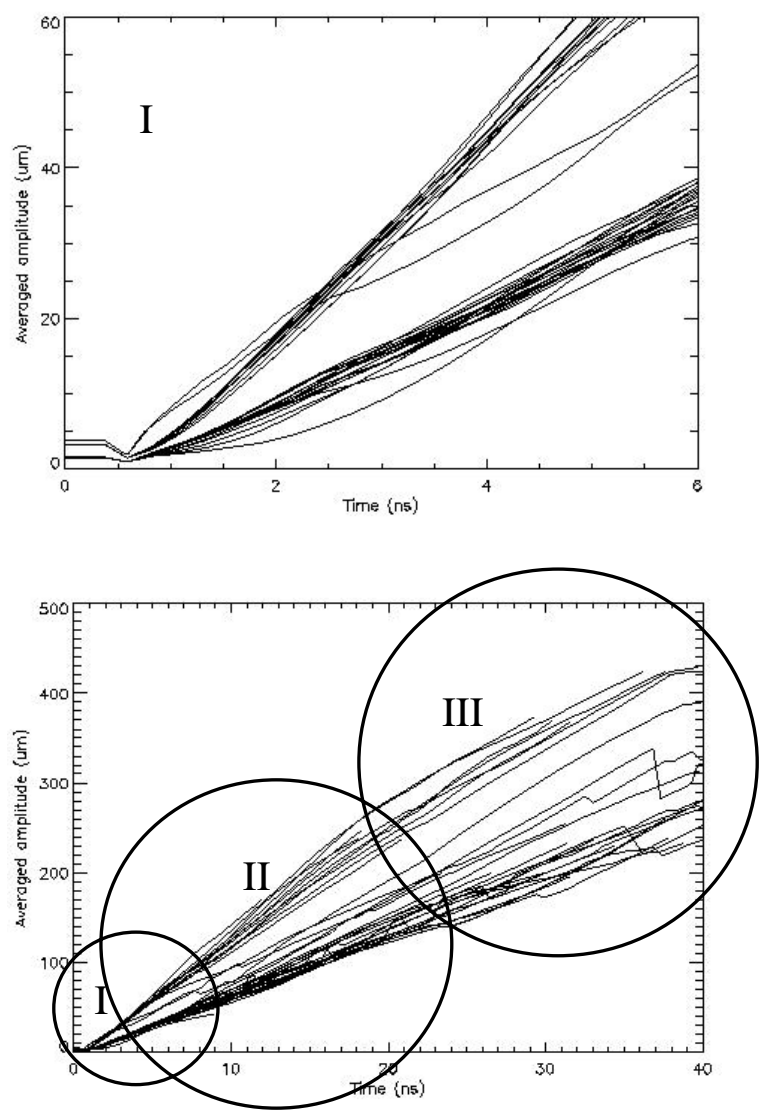

Figure 7: Three phases of instability growth. Phase I: The early-time (linear, early nonlinear, and into nonlinear) phase is dominated by RM for about $1 \mathrm{~ns}$. The growth rate is determined by the most unstable mode, and the inverse cascade is initiated. Phase II: Changes in growth rate result in strong dependence on spectral details in addition to the initial $\mathrm{a}_{\mathrm{rms}}$. Phase III: Mode 1 emerges as the dominant transverse scale after up to 5 bubble merger generations. The acceleration profile introduces an "effective box size". The asymptotic velocity depends on amplitude, time in addition to the transverse scale and the degree of mix in the layer.

well correlated with small changes in the initial rms amplitude or with the spectral shape.

Phase III begins when mode 1 emerges as the dominant transverse scale after up to five bubble merger generations. After this scale is reached, the inverse cascade is halted and the growth is no longer self-similar-like. One would tend to conclude at 


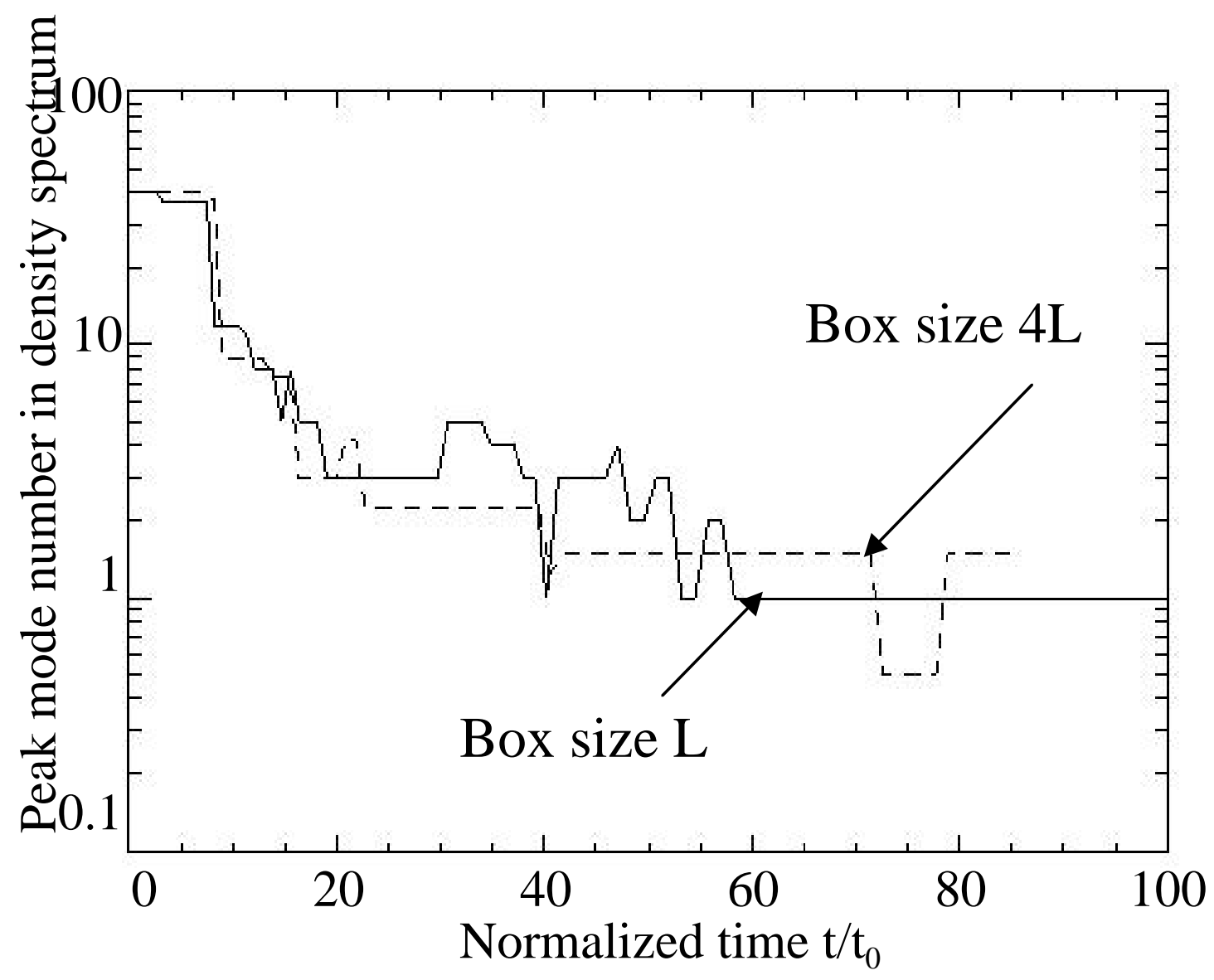

Figure 8: "Effective box size" imposed by the decaying nature of the drive coupled with the finite duration of the experiment. With a box-size of $\mathrm{L}=200 \mu \mathrm{m}$, mode 1 emerges as the dominant mode at about $\mathrm{t} / \mathrm{t}_{0}=60(24 \mathrm{~ns})$. Even when the width of computational domain is quadrupled, longer-wavelength modes do not dominate at late times. After $25 \mathrm{~ns}$, the time required to generate lower 1-modes exceeds the time remaining in the experiment. Both cases use the same narrow gaussian spectral shape.

this point that this signals the end of the calculation's range of validity because the computational box has been "filled" so that end effects corrupt further evolution. In fact, calculations run with twice or even four times the nominal box size generally show no significant change in the perturbation growth history and or the late-time dominant transverse scale (see Fig. 8). We attribute this to the decaying nature of the driving acceleration. As the acceleration approaches zero, the time to generate larger 
scales (which even with constant acceleration take longer to form than shorter scales) tends to infinity. Thus the decaying acceleration profile coupled with the finite experiment duration introduces an "effective box size" even in the absence of an experimentally or computationally-imposed physical box size.

\section{Quasi-self-similar regime}

The main point in plotting all the amplitude trajectories together on one plot (Fig. 5) is to show that they generally diverge in time rather than converge as one might expect during approach to a stationary self-similar bubble distribution. That is, there is no apparent approach to a self-similar regime independent of the initial conditions. This is true even if one considers only those runs from the lower family with only the short wavelength component and with initial rms amplitude of about $0.25 \mu \mathrm{m}$. If one assumes self-similarity (ie that the characteristic transverse scale is a constant fraction of the mix-width) and takes into account the time-dependence of the acceleration and the large-scale velocity gradient present in the zero-order hydro, then the spike and bubble growth in each run can be characterized by a constant factor $\alpha$ (the $\alpha$ of $h(t)=\alpha \mathrm{Agt}^{2}$ models). In a true self-similar regime, the value of this parameter should be a universal constant with weak (if any) dependence on Atwood number. ${ }^{33}$ Within the $\mathrm{a}_{\mathrm{rms}}=0.25 \mu \mathrm{m}$, shorts only subgroup, we instead find that $\alpha_{\text {bubble }}$ varies over a range of about $0.035-0.065$ while $\alpha_{\text {spike }}$ varies over $0.050-0.100$. This nearly covers the entire range of values reported from different experiments and simulations (see, for example, Ref. 34 and references therein), though it falls somewhat short of the values reported for spike growth at this Atwood number (as 


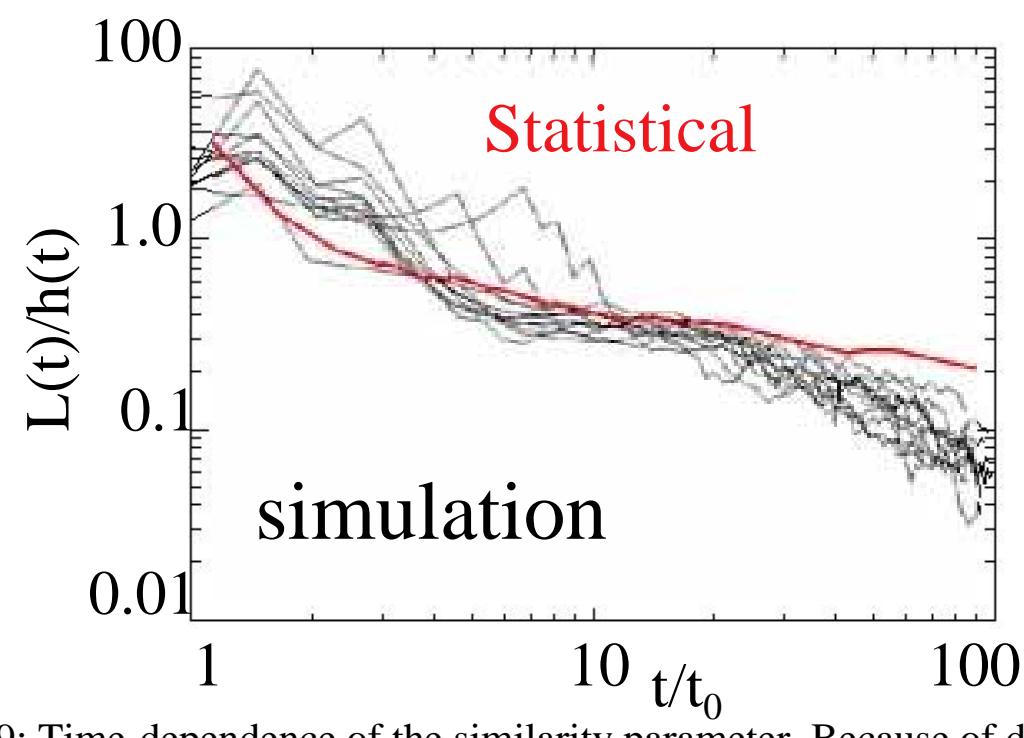

Figure 9: Time-dependence of the similarity parameter. Because of decompression, the similarity parameter decays in time rather than approaching a constant asymptotic value. This behavior is well-predicted by a statistical-mechanics bubble merger model that includes decompression and the time-dependence of the drive.

high as 0.120 ). Thus the assumption of self-similarity does not lead to a useful means of characterizing the instability growth. Instead, memory of the initial conditions is retained throughout the experiment at least in the mix width. Rather than approaching a constant, the similarity parameter (the ratio of characteristic transverse scale to perturbation amplitude) decays in time. The time dependence of the similarity parameter is shown in Fig. 9 for various initial conditions, including examples of each spectral type included in the study, with rms-amplitudes varying over three orders of magnitude. A narrow gaussian with four times the nominal box width and three flat spectrum calculations with twice the nominal box width are included to show that variations in system size (boundary effects) do not change the general behavior of the similarity parameter.

Because of decompression and drive decay, the asymptotic bubble and spike velocities depend on the amplitude and time as well as on the transverse scale and the 
degree of mix in the layer. The amplitude dependence arises because of the velocity gradient, which is approximately proportional to $\Delta \mathrm{r} / \mathrm{t}$ and characteristic of a rarefaction fan. Here $\Delta \mathrm{r}$ is the distance in the parallel direction between any two points, in particular the distance from the unperturbed interface to the position of the spike or bubble tip. Thus the contribution of material decompression and stretching to the spike or bubble velocity at a given time is proportional to its amplitude.

We have extended an existing statistical-mechanics bubble merger model ${ }^{35}$ to include decompression and the time-dependence of the drive. ${ }^{36}$ Details of the model are presented in Ref. 36. We note here only that the model prediction (the red line in Fig. 9) also shows a decaying similarity parameter and agrees well with the simulations.

However, there is apparently loss of transverse spectral information and a period of "quasi-self-similar" growth. This is illustrated in two ways Fig. 10. Figure 10(a) shows a time series of log density plots from a small initial amplitude simulation with a flat spectrum (modes 4-80). During this period, which covers the first $10 \mathrm{~ns}$ of growth, the inverse cascade to progressively larger scales is apparent. In Fig. 10(b), the images are rescaled so that the mix-width appears approximately constant in time. The similarity in interface structure in the rescaled images shows that the ratio of dominant transverse scale to mix width does not change much over this time interval. Since the value of this ratio does tend to slowly decrease over time as the material decompresses, we refer to this as a "quasi-self-similar" regime.

Figure 10(c) shows log density plots from simulations with different initial spectral types at early and intermediate times (2.4 and $11.5 \mathrm{~ns})$. Early on, the 


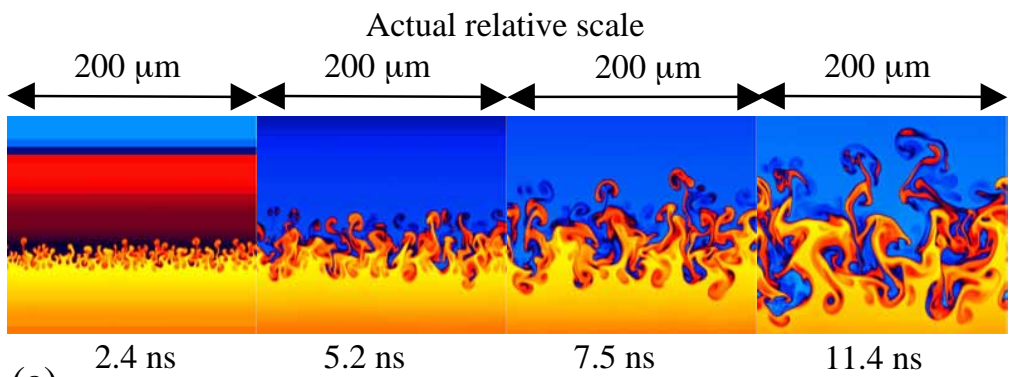

(a) $\quad 2.4 \mathrm{~ns} \quad 5.2 \mathrm{~ns} \quad 7.5 \mathrm{~ns}$ Rescaled images show self-similar-like growth

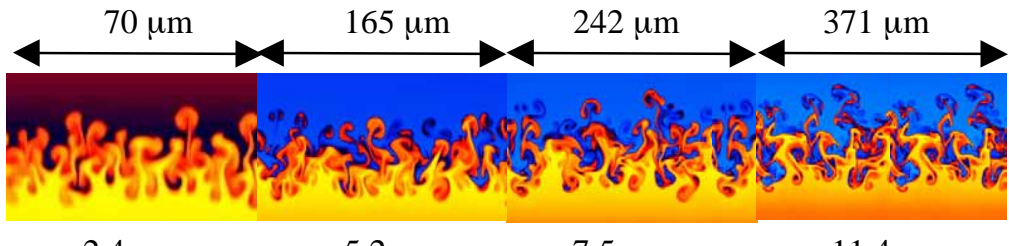

(b)

$$
\begin{array}{llll}
2.4 \mathrm{~ns} & 5.2 \mathrm{~ns} & 7.5 \mathrm{~ns} & 11.4 \mathrm{~ns}
\end{array}
$$

$\begin{array}{lccc}\text { Flat } & \text { Flat } & \text { Narrow gaussian } & \text { Hyperbolic } \\ =2.5 \mu \mathrm{m} & \mathrm{a}_{\mathrm{m}}=0.25 \mu \mathrm{m} & \mathrm{a}_{\mathrm{m}}=0.25 \mu \mathrm{m} & \mathrm{a}_{\mathrm{rms}}=0.25 \mu \mathrm{m}\end{array}$ $\mathrm{a}_{\mathrm{rms}}=2.5 \mu \mathrm{m} \quad \mathrm{a}_{\mathrm{rms}}=0.25 \mu \mathrm{m} \quad \mathrm{a}_{\mathrm{rms}}=0.25 \mu \mathrm{m} \quad \mathrm{a}_{\mathrm{rms}}=0.25 \mu \mathrm{m}$

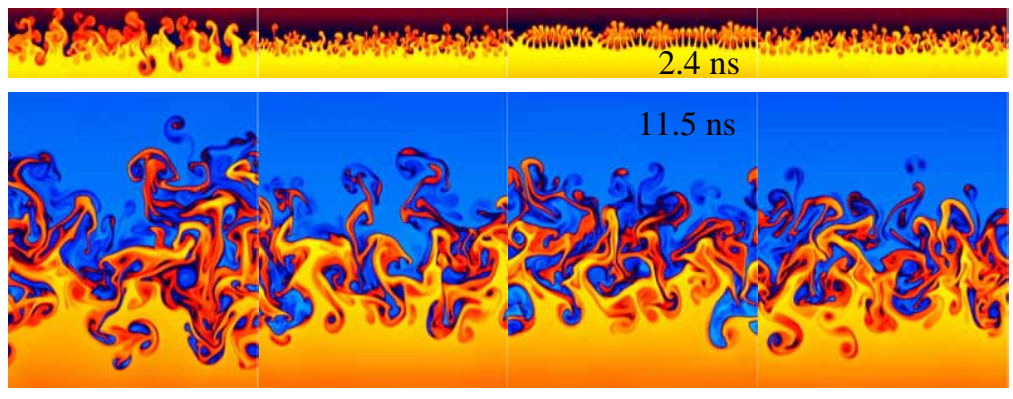

(c)

Figure 10: Quasi-self-similar regime. (a) A time series of log density plots from a small initial amplitude simulation with a flat spectrum (modes 4-80) shows the inverse cascade to progressively larger scales. (b) The same images are rescaled so that the mix-width appears approximately constant in time. The similarity in interface structure in the rescaled images shows that the ratio of dominant transverse scale to mix width does not change much over this time interval. (c) The loss of transverse spectral information is illustrated by log density plots from simulations with different initial spectral types at early and intermediate times (2.4 and $11.5 \mathrm{~ns})$. Early on, the interface structure is clearly correlated with the initial conditions. The later-time images appear far more similar to one other. 
interface structure is clearly correlated with the initial conditions. In particular, mode 40 is apparent in the early-time narrow gaussian image. The later-time images, on the other hand, appear far more similar to one other. As was noted previously, there is a wide spread in the late time amplitude growth, but the dependence on spectral type within that spread is generally not much larger than the variation between different realizations with the same spectral shape.

The loss of transverse spectral information is illustrated more quantitatively in Fig. 11, which compares average $1 \mathrm{D}$ density fluctuation spectra at $\mathrm{t}=0$ and $\mathrm{t}=10 \mathrm{~ns}$ for a variety of short-wavelength spectral shapes. The density fluctuation is defined by $\delta \rho(z)=\rho(z)-\langle\rho(z)>$, where $<\rho(z)>$ is the $\mathrm{z}$-dependent transverse density average. Each spectrum shown in Fig. 11 is the average of several (typically nine) 1D spectra evenly spaced throughout the interior of the mix region. Despite significant differences in the initial spectral shapes and rms-amplitudes, all transverse spectral information above about mode 10 has been lost by $10 \mathrm{~ns}$, and memory of the initial conditions is retained only in the amplitudes of the long wavelength modes. For runs with the same initial rms-amplitude, the low-mode end of the spectrum is also very similar, suggesting that only memory of the initial amplitude and not the spectral shape has been retained. An inertial range with Kolmogorov $\mathrm{k}^{-5 / 3}$ scaling is visible between modes thirty and eighty.

Transverse and parallel turbulent kinetic energy spectra and velocity fluctuation spectra from the same simulations are shown in Figs. 12 and 13, respectively. The fluctuating components of the energy and velocity are defined in the same way as the density fluctuation: $\delta \mathrm{KE}_{\mathrm{x}, \mathrm{z}}(\mathrm{z})=\mathrm{KE}_{\mathrm{x}, \mathrm{z}}(\mathrm{z})-<\mathrm{KE}_{\mathrm{x}, \mathrm{z}}(\mathrm{z})>$ and 

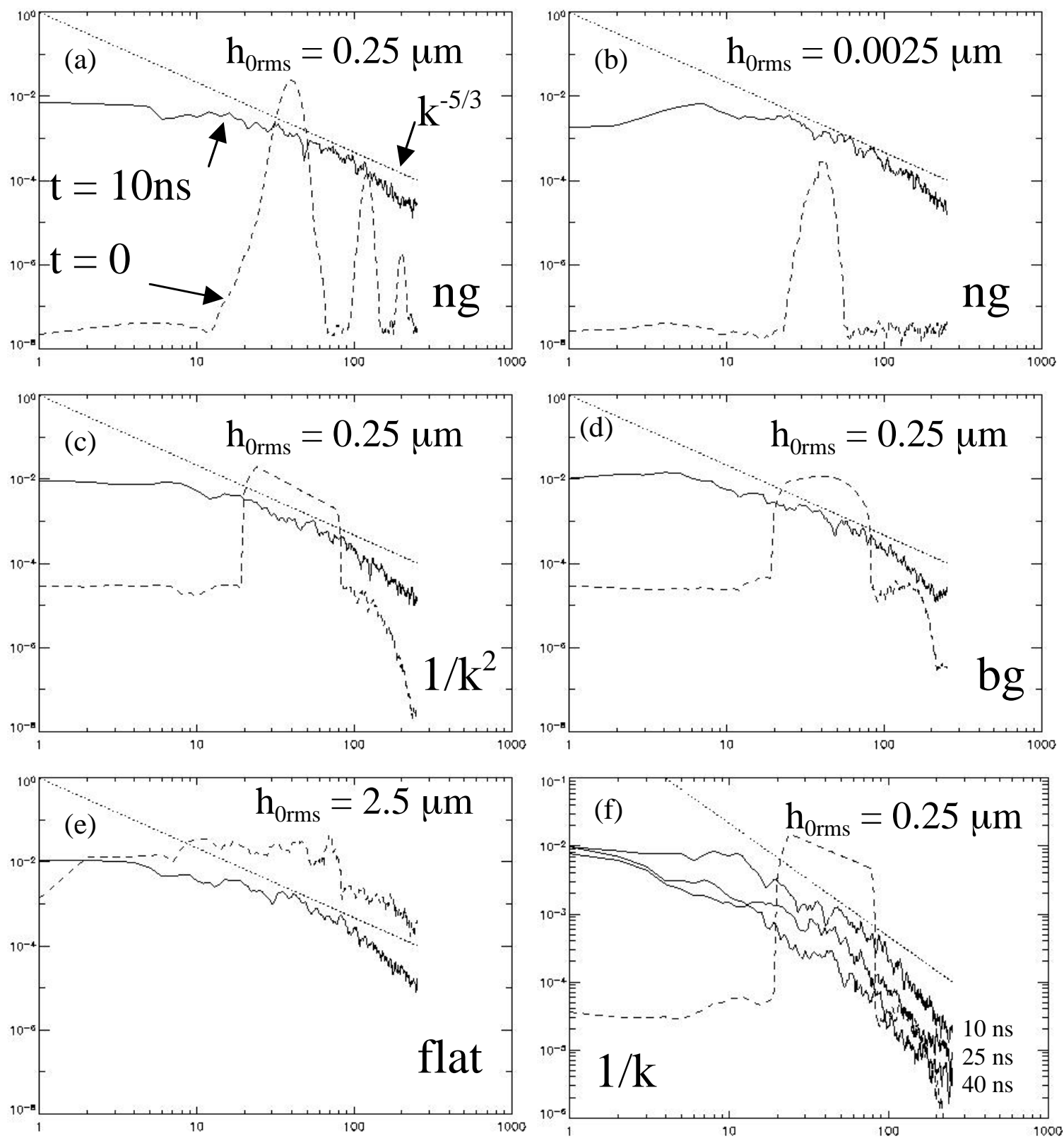

Figure 11: Loss of transverse spectral information. Density spectra at $t=0$ and $t=10$ ns for a variety of short-wavelength spectral shapes: (a) Narrow gaussian with $\mathrm{h}_{0 \mathrm{rms}}=$ $0.25 \mu \mathrm{m}$, (b) narrow gaussian with $\mathrm{h}_{0 \mathrm{rms}}=0.0025 \mu \mathrm{m}$, (c) $1 / \mathrm{k}^{2}$ spectrum with $\mathrm{h}_{0 \mathrm{rms}}=$ $0.25 \mu \mathrm{m},(\mathrm{d})$ broad gaussian with $\mathrm{h}_{0 \mathrm{rms}}=0.25 \mu \mathrm{m}$, and (e) flat (modes 4-80) with $\mathrm{h}_{0 \mathrm{rms}}$ $=2.5 \mu \mathrm{m}$. (f) hyperbolic $(1 / \mathrm{k})$ spectrum with $\mathrm{h}_{0 \mathrm{rms}}=0.25$. By $10 \mathrm{~ns}$, all transverse spectral information above about mode 10 has been lost, and memory of the initial conditions is retained only in the amplitudes of the long wavelength modes. For runs with the same initial rms-amplitude, the low-mode end of the spectrum is also very similar, suggesting that only memory of the initial amplitude and not the spectral shape has been retained. In (f), we include spectra at 25 and $40 \mathrm{~ns}$. After establishment of the quasi-self-similar regime, there is little change in the spectra shape except for a steeper slope at the lowest modes. 

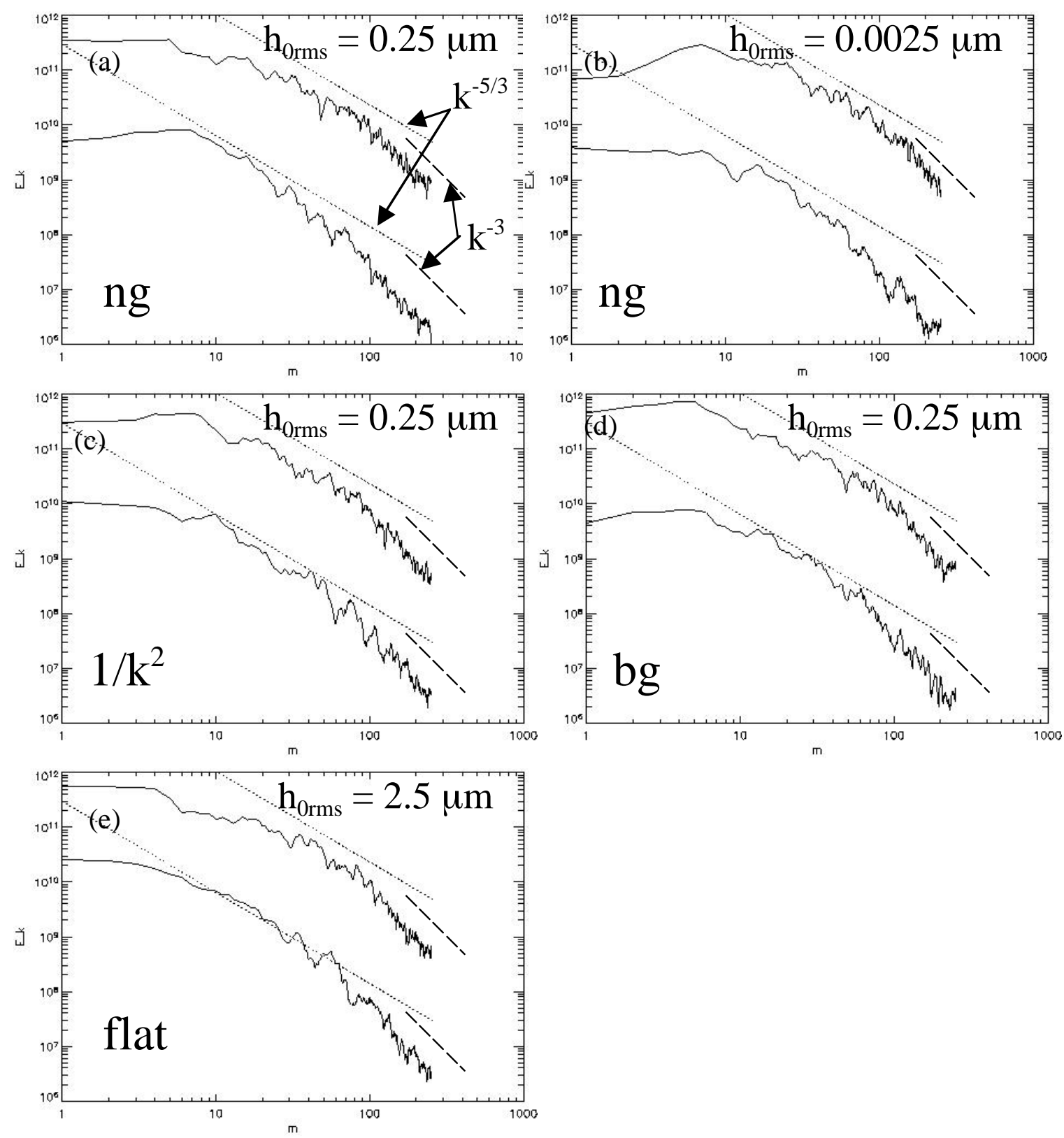

Figure 12: Transverse and parallel turbulent kinetic energy spectra at $10 \mathrm{~ns}$. The upper solid curve is the parallel directed turbulent kinetic energy and the lower solid line is the transverse directed turbulent kinetic energy. (a) Narrow gaussian with $\mathrm{h}_{0 \mathrm{rms}}$ $=0.25 \mu \mathrm{m}$, (b) narrow gaussian with $\mathrm{h}_{0 \mathrm{rms}}=0.0025 \mu \mathrm{m}$, (c) $1 / \mathrm{k}^{2}$ spectrum with $\mathrm{h}_{0 \mathrm{rms}}=$ $0.25 \mu \mathrm{m}$, (d) broad gaussian with $\mathrm{h}_{\text {orms }}=0.25 \mu \mathrm{m}$, and (e) flat (modes 4-80) with $\mathrm{h}_{\text {orms }}$ $=2.5 \mu \mathrm{m}$. By $10 \mathrm{~ns}$, the spectral shape depends weakly on the initial conditions, and both transverse and parallel components exhibit a limited $\mathrm{k}^{-5 / 3}$ inertial range. In most cases, the high-mode end of the spectrum is somewhat steeper than a $\mathrm{k}^{-3}$ scaling. 

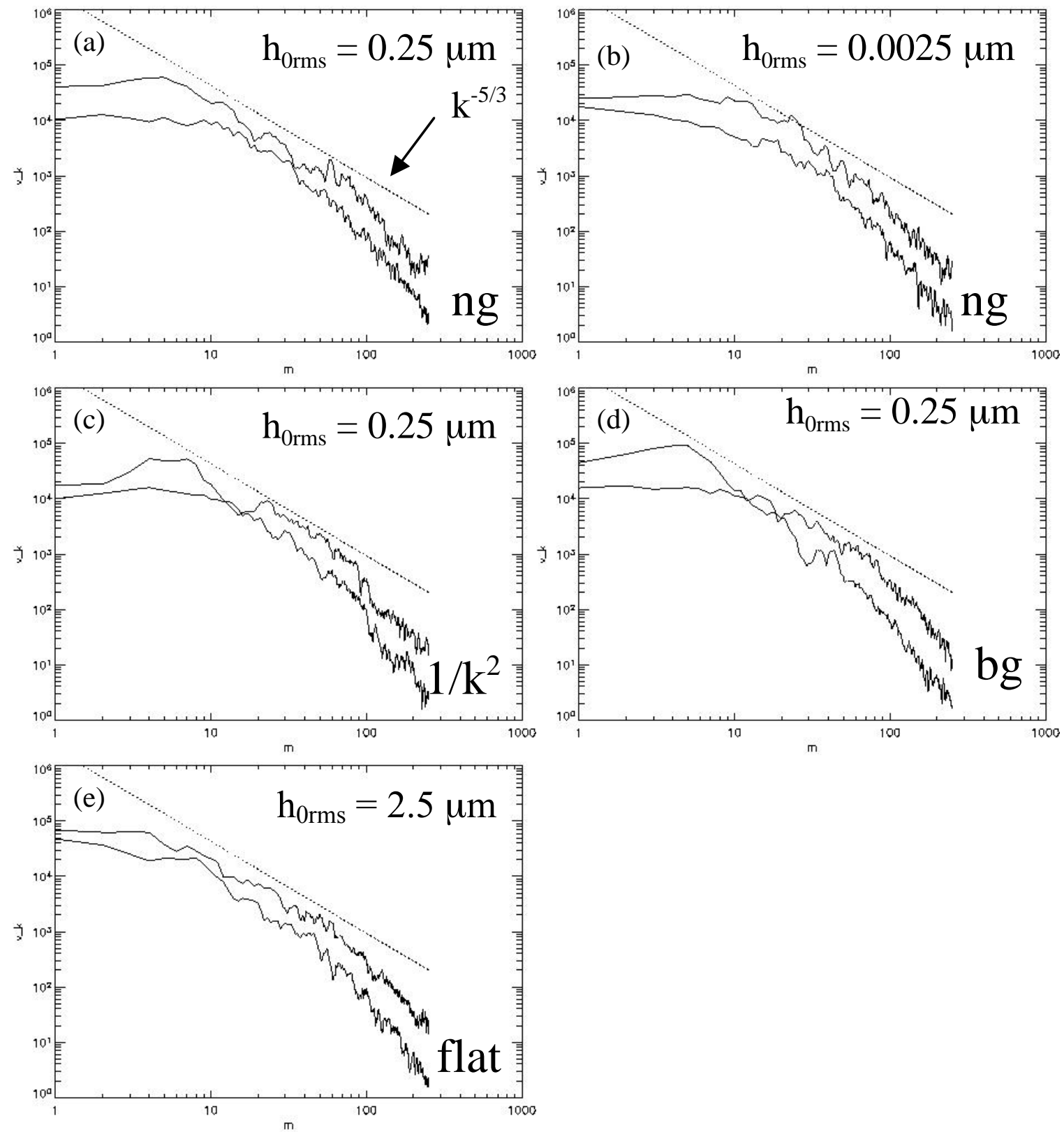

Figure 13: Transverse and parallel velocity fluctuation spectra at $10 \mathrm{~ns}$. The upper solid curve is the parallel velocity fluctuation and the lower solid line is the transverse velocity fluctuation. (a) Narrow gaussian with $\mathrm{h}_{0 \mathrm{rms}}=0.25 \mu \mathrm{m}$, (b) narrow gaussian with $\mathrm{h}_{0 \mathrm{rms}}=0.0025 \mu \mathrm{m}$, (c) $1 / \mathrm{k}^{2}$ spectrum with $\mathrm{h}_{0 \mathrm{rms}}=0.25 \mu \mathrm{m}$, (d) broad gaussian with $\mathrm{h}_{0 \mathrm{rms}}=0.25 \mu \mathrm{m}$, and (e) flat (modes 4-80) with $\mathrm{h}_{0 \mathrm{rms}}=2.5 \mu \mathrm{m}$. By $10 \mathrm{~ns}$, the spectral shape depends weakly on the initial conditions, and both transverse and parallel components exhibit a limited $\mathrm{k}^{-5 / 3}$ inertial range. 
$\left|\delta \mathrm{v}_{\mathrm{x}, \mathrm{z}}(\mathrm{z})\right|=\left|\mathrm{v}_{\mathrm{x}, \mathrm{z}}(\mathrm{z})-<\mathrm{v}_{\mathrm{x}, \mathrm{z}}(\mathrm{z})>\right|$, where " $<>$ " again denotes transverse average and $\mathrm{KE}_{\mathrm{x}, \mathrm{z}}$ $=\rho \mathrm{v}_{\mathrm{x}, \mathrm{z}}{ }^{2}$. In each case, the upper solid curve is the parallel (z) component and the lower solid line is the transverse (x) component. By $10 \mathrm{~ns}$, the spectral shape depends weakly on the initial conditions, and both transverse and parallel components exhibit a limited $\mathrm{k}^{-5 / 3}$ inertial range. In most cases, the high-mode end of the spectrum is reasonably well approximated by a $\mathrm{k}^{-3}$ scaling.

Scaling laws for the energy spectrum of stationary 2D "turbulence" were first put forward by Kraichnan, who considered an unbounded system into which energy is uniformly injected at some wavenumber $\mathrm{k}_{\mathrm{inj}}{ }^{37}$ At scales larger than the injection scale, he predicted that an inverse energy cascade driven by vortex merger would result in a Kolmogorov $\mathrm{k}^{-5 / 3}$ scaling. Below the injection scale, a forward enstrophy cascade would give $\mathrm{E}(\mathrm{k}) \sim \mathrm{k}^{-3}$. Recent experiments using flowing soap films agree with Kraichnan's predictions at both high and low wavenumbers. ${ }^{38}$

Thus our observation of an inertial range with $\approx-5 / 3$ scaling at lower mode numbers indicates a transition to $2 \mathrm{D}$ turbulence. At higher mode numbers, the energy spectra are typically fall of somewhat steeper than $\mathrm{k}^{-3}$. After establishment of the quasi-self-similar regime, there is little change in the spectra shape except for a steeper slope at the lowest modes [see Fig. 11(f)].

The RT instability can in principle inject energy into the system at all scales in the density spectrum, but the growth of under-resolved modes is inhibited. The upper end of the inertial range in the energy spectra in Fig. 12 corresponds to injection scales at modes resolved by 3-8 ppw. This is certainly insufficient to resolve the forward enstrophy cascade, so it is not surprising that the spectra tend to fall off 

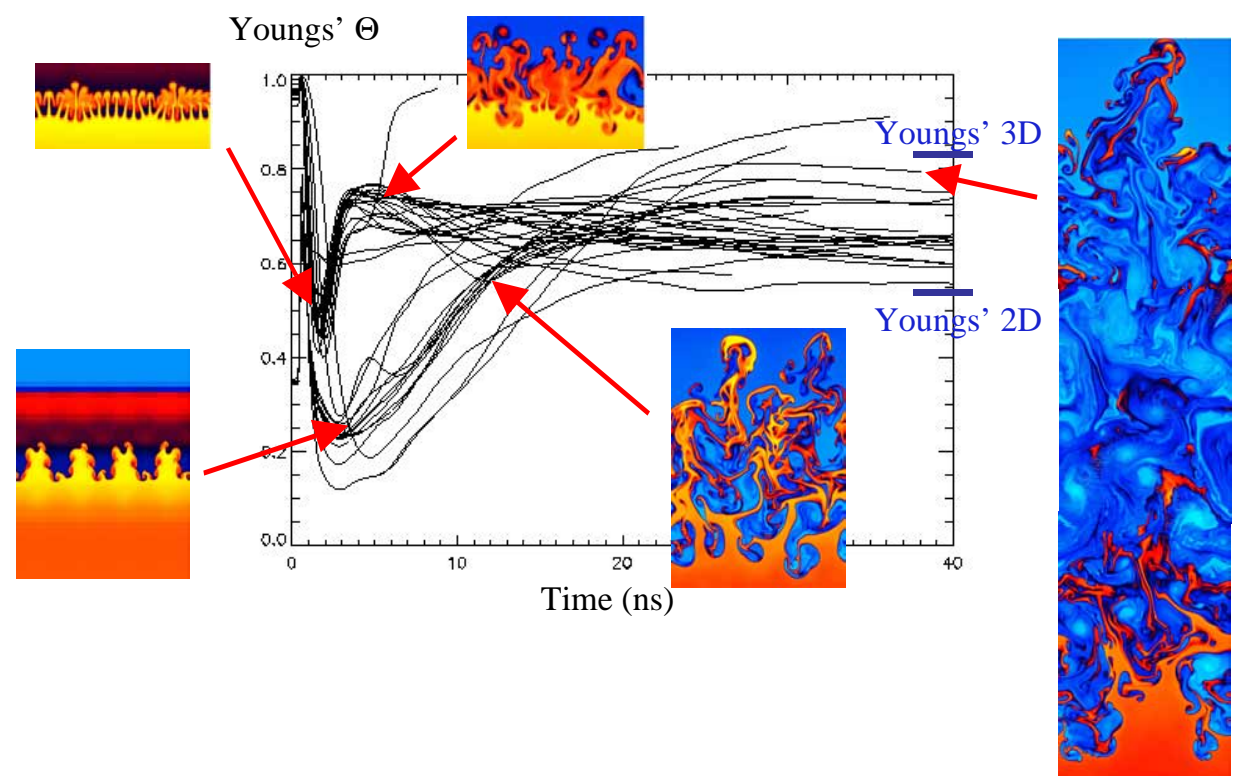

Figure 14: Degree of mixedness for all 52 2D simulations included in Fig. 5. Transition to quasi-self-similar regime results in an increase in mixedness. For a similar density ratio, Youngs* reports $\Theta \approx 0.83$ in $3 \mathrm{D}$ and $\Theta \approx 0.54$ in $2 \mathrm{D}$ [D.L. Youngs, Lasers and Particle Beams, 12(4), 725 (1994)].

somewhat faster than $\mathrm{k}^{-3}$ at higher mode numbers.

The degree of "mixedness", which Youngs calls the molecular mix fraction, ${ }^{33}$ provides a good measure of when the transition to the quasi-self-similar regime takes place. The mixing parameter is defined by

$$
\Theta \equiv \frac{\int\langle f(1-f)\rangle d z}{\int\langle f\rangle d z \int\langle 1-f\rangle d z},
$$

where $\mathrm{f}$ is the volume fraction of either of the two fluids, the averaging is done in the transverse direction, and the integral is performed in the parallel direction through the extent of the mix region. The mixing parameter time histories from all 52 2D simulations included in Fig. 5 are shown in Figure 14. Again, most of the curves fall into one of two families. The upper family contains runs without the large amplitude 
mode 4, while the lower family consists of all the runs with the large mode 4 . In both cases, there is a clear transition from a state that is not well mixed to a state with higher degree of mixedness that tends to an asymptotic value between about 0.6 and 0.8. With no large mode 4 , this transition occurs within a few ns, and corresponds to the transition to the quasi-self-similar regime. The same transition occurs when the large mode 4 is included in the initial spectrum, but the time to transition is several times longer. Thus the presence of the long wavelength mode delays the transition to a turbulent-like state. Comparison of Fig. 14 with amplitude history plots shows that, in addition to the increase in mixedness, transition to the quasi-self-similar regime is marked by a decrease in the spike velocity and often an increase in the bubble velocity. The spike velocity decreases in particular for the runs with large-amplitude mode 4, where the transition is associated with the breakup of the primary spikes. This breakup allows the spikes' parallel energy to be diverted into the transverse direction and results in a decrease in the effective Atwood number in the mix region. When the initial spectrum gives an array of nearly identical bubbles, transition can allow for bubble competition and the generation of larger scales, resulting in an increase in the velocity of the bubble front.

The effective Atwood number reduction experienced by the spikes due to increased mixing after their breakup results in an increase in the bubble to spike amplitude ratio, shown in Fig. 15 for several representative cases. In the single-mode (mode 4) case, the amplitude ratio approaches a value of about 0.48 . This is just slightly higher than the value of $\sqrt{\rho_{b} / \rho_{s}} \approx 0.42$ predicted by a buoyancy-drag model assuming that the spike reaches terminal velocity early on. ${ }^{5,39}$ With the spike 


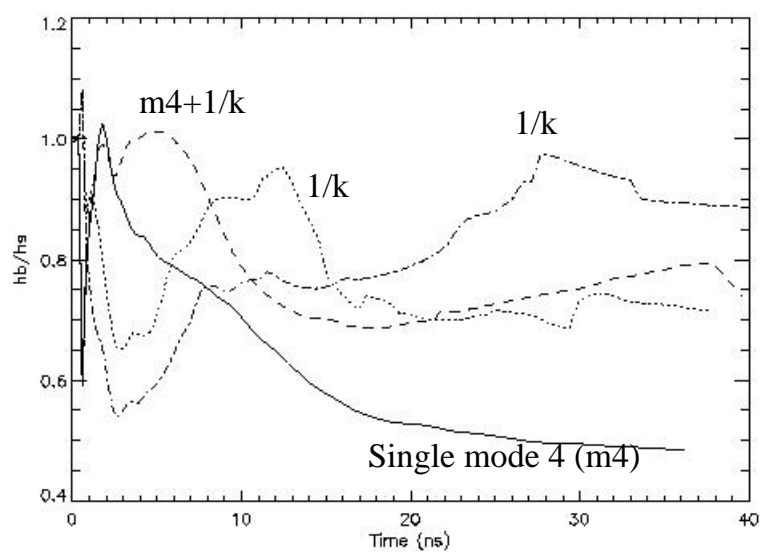

Figure 15: Bubble to spike amplitude ratio: In the single mode 4 case, the amplitude ratio approaches a value of about 0.48 . This is just slightly higher than the value of 0.42 predicted by a buoyancy-drag model assuming that the spike reaches terminal velocity early on. With the spike interaction and breakdown associated with transition to the quasi-self-similar regime, the amplitude ratio is much closer to unity and typically greater than 0.7 at late times.

interaction and breakdown associated with transition to the quasi-self-similar regime, the amplitude ratio is much closer to unity and typically greater than 0.7 at late times. The same tendency towards spike-bubble amplitude symmetry due to spike breakup was reported by Youngs, who also noted an associated weak dependence of $h_{b} / h_{s}$ on the density ratio. ${ }^{33}$

In a true self-similar regime (at least for the case of incompressible flow, no RM component, and constant acceleration - all of which are violated here), the posttransition mixedness should be a universal constant depending only on the Atwood number. ${ }^{33}$ For a density ratio similar to ours (and with very weak density ratio dependence), Youngs found in his 3D calculations that the asymptotic degree of mix increases at higher resolution where the inertial range is better resolved. Extrapolating the observed trend to infinite resolution, he reports a value of about 0.83 in $3 \mathrm{D}$ and 0.54 in $2 \mathrm{D} .{ }^{40}$ Cook et al. find similar values in their high resolution, classical RT 


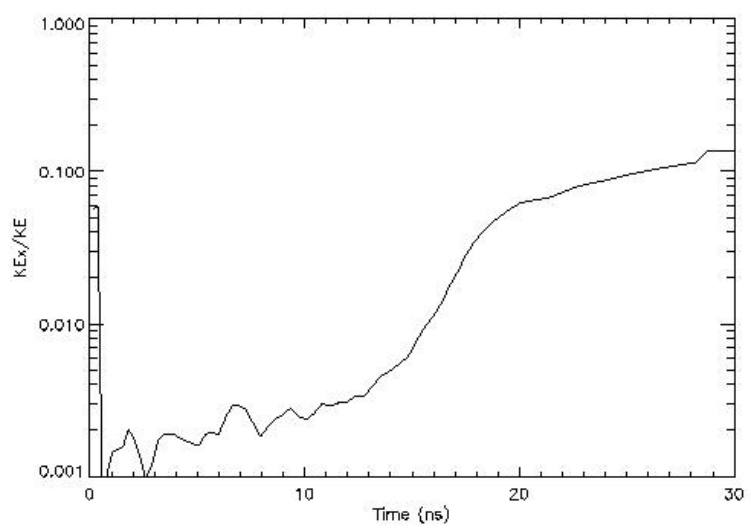

Figure 16: Ratio of transverse to total kinetic energy, integrated over the mix region, from the single mode 4 simulation. Below $15 \mathrm{~ns}$, the relative transverse kinetic energy increases slowly. The breakup of the mode 4 spikes at 15-20 ns corresponds to a much faster order of magnitude increase.

calculations. ${ }^{41}$ The values we find are distributed throughout this range, and are consistently higher than the reported $2 \mathrm{D}$ value.

Finally, we note that there is no true turbulent mixing transition ${ }^{42}$ in the simulations. This is to be expected due to the low effective Reynolds number and the 2D nature of the simulations. Three dimensional turbulence is characterized by a forward cascade of energy to smaller scales where it is eventually dissipated. Vortex stretching, which is the mechanism of coupling to smaller scales, is fundamentally a $3 \mathrm{D}$ process and therefore absent in 2D systems. However, the abrupt increase in mixedness observed in the $2 \mathrm{D}$ calculations and associated with the onset of strong spike interaction and breakup is reminiscent of a turbulent mixing transition. While spikes grow without interacting with one another, their energy is directed almost entirely in the parallel direction. When they interact and breakup, a significant fraction of their energy is diverted into the parallel direction (see Fig. 16) and smaller scales are generated via the Kelvin Helmholtz $(\mathrm{KH})$ instability. ${ }^{43}$ Because of this 
forward cascade, a 2D system undergoing spike breakup in a sense temporarily mimics 3D turbulence, and there is an associated "2D turbulent mixing transition".

\section{Resolution study}

A resolution study was performed in order to verify adequate convergence of the growth rates and interface structure at the nominal resolution of 512 points across the computational domain (512 ppb). The resolution was varied from 128 to 2048 cells per box width (ppb), or from four times less than to four times greater than the nominal resolution. Log density plots [Fig. 17(a)] and perturbation amplitude histories [Fig. 17(b)] from a series of calculations initialized with the same narrow gaussian (shorts only) spectrum suggest that the mix width and interface structure are reasonable well-resolved at $256 \mathrm{ppb}$. Even at $128 \mathrm{ppb}$, the mix width is only reduced by $15-25 \%$ relative to the highest resolution case. This is impressive considering that, at $128 \mathrm{ppb}$, the initially dominant mode (mode 40) is resolved to only 3.2 points per wavelength (ppw). Considering the extreme drive strength and only marginally linear initial conditions, the perturbation becomes nonlinear very quickly and the observed fast convergence is perhaps due to the fast generation of larger, better resolved scales due to mode coupling ${ }^{30}$ and nonlinear interactions among spikes. ${ }^{43}$

A plot of the mixing parameter [Fig. 17(c)] as a function of time shows that, in contrast to Youngs's 3D calculations, ${ }^{33}$ there is more mixing at lower resolution where the numerical diffusion is greater. The algorithm used to identify the spike and bubble positions is based on the product of the volume fractions of the two fluids averaged over the transverse direction (the mix width is by definition bounded by the 


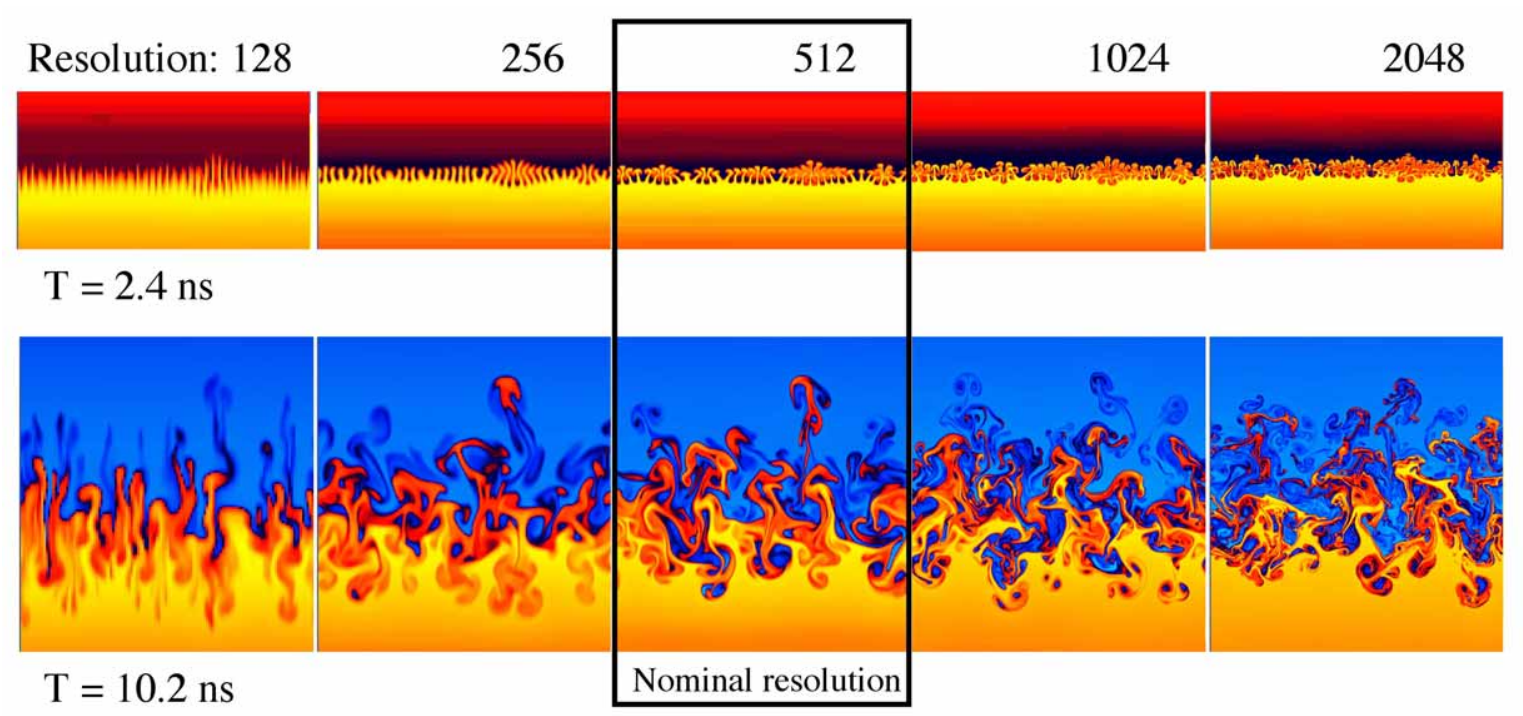

(a)

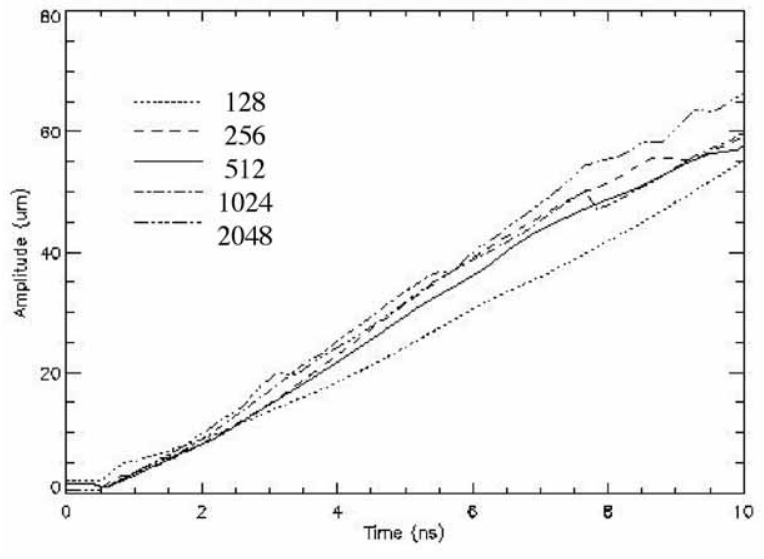

(b)

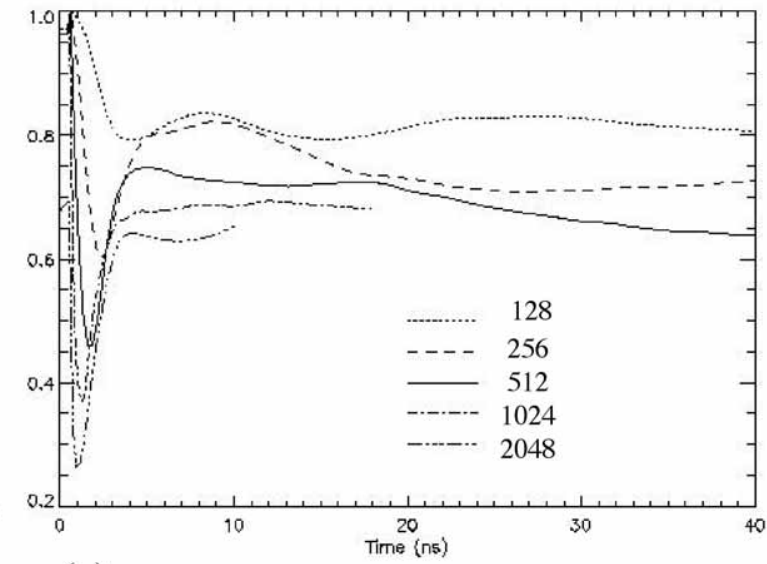

(c)

Figure 17: Resolution study: The resolution is varied from 128 to 2048 cells per box width (ppb), or from four times less than to four times greater than the nominal resolution of $512 \mathrm{ppb}$. (a) Log density plots and (b) perturbation amplitude histories suggest that the interface structure is reasonable well-resolved at $256 \mathrm{ppb}$. (c) A plot of the mixing parameter as a function of time shows that there is more mixing at lower resolution where the numerical diffusion is greater.

$2.5 \%$ points). When the outlying spikes are more diffuse due to decreased resolution, the algorithm identifies an edge that corresponds to a smaller spike amplitude. This is the primary reason for the correlation between lower resolution and reduced amplitude. 
Density, directed kinetic energy, and velocity spectra from a calculation at the highest resolution (2048 ppb) are shown in Fig. 18. Results from a simulation at the nominal $512 \mathrm{ppb}$ resolution with the same narrow gaussian spectral shape are included for comparison. For each quantity, the low-k end of the inertial range is about the same at either resolution. The high-k end extends to much higher mode numbers in the $2048 \mathrm{ppb}$ case, consistent with its higher numerical Reynolds number and resulting in an inertial range that spans about one decade. The minimum energy injection scale is at about mode 500-700, which again says that the driving instability injects energy into modes resolved by at least 3-5 ppw.

\section{E. Dependence of transition time on initial conditions}

We have already seen how several of the factors listed in Table 1 affect the large-scale instability evolution. In all stages of the instability, larger initial amplitudes give larger amplitude later on. The initial shape of the short-wavelength spectral component has little effect on the late-time growth, including the time to transition to a turbulent-like state. This statement assumes, however, that the initial spectrum includes multiple modes that are not both commensurate and in phase with

one another. Without numerical or physical sources of random noise, such spectra can only lead to a limited inverse cascade that gives rise to stable periodic arrays of bubbles.

We have also seen that there is a correlation between transition to the quasiself-similar regime, which is characterized by a loss of transverse spectral information, and a "2D mixing transition" to a state characterized by a mixedness of 

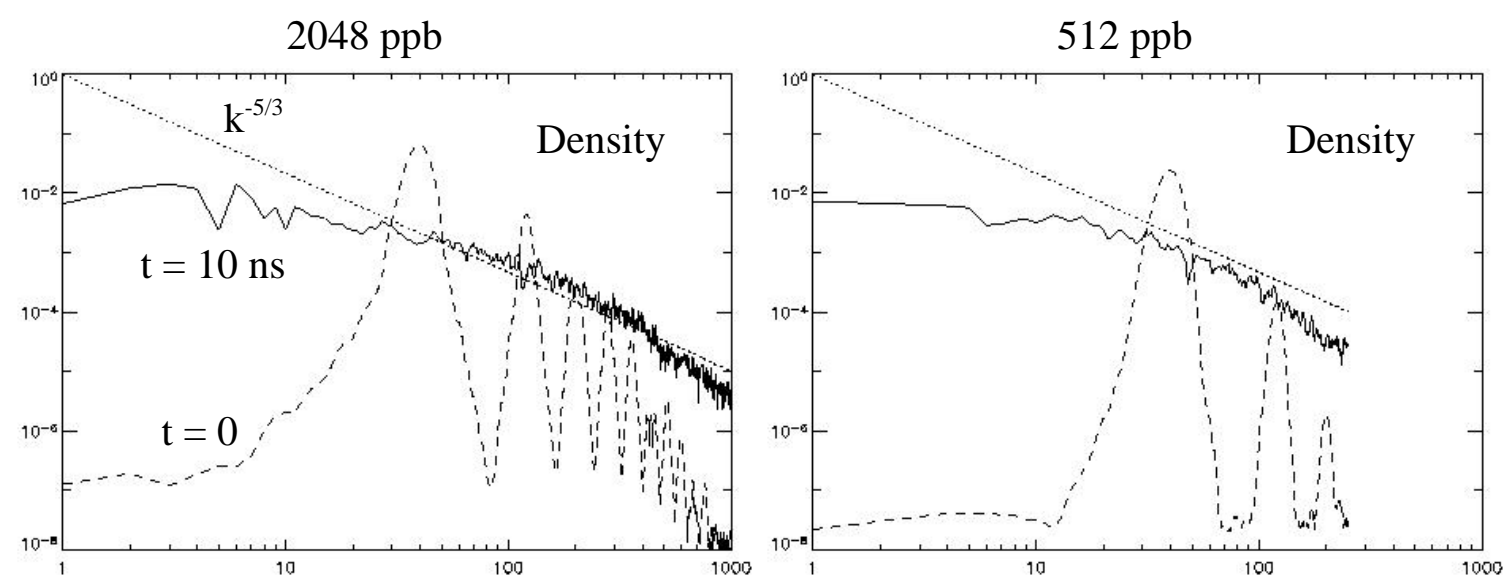

(a)
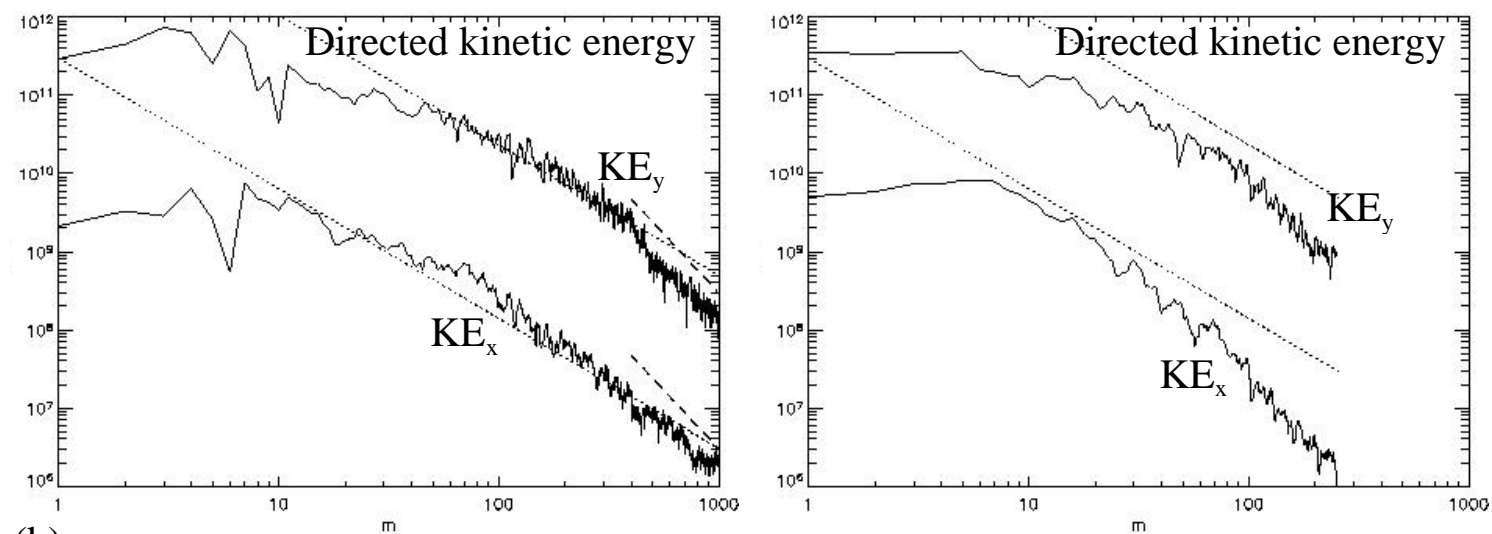

(b)
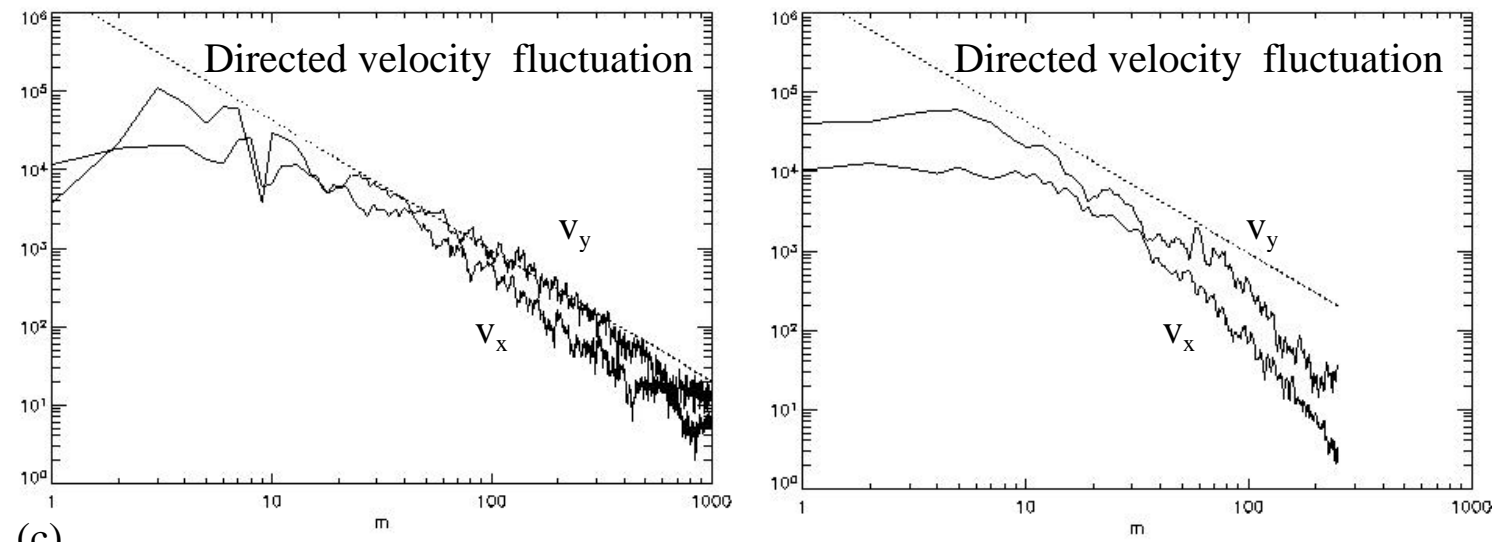

(c)

Figure 18: (a) Density, (b) directed turbulent kinetic energy, and (c) directed velocity fluctuation spectra at 0 and $10 \mathrm{~ns}$ for highest resolution (2048 ppb). The initial spectrum is narrow gaussian with randomized amplitudes and phases. Spectra from a $512 \mathrm{ppb}$ case with the same spectral shape are included for comparison. In the energy spectra, dashed lines denote the $\mathrm{k}^{-3}$ scaling associated with a forward enstrophy cascade in 2D turbulence. 
$0.6-0.8$. What remains is to determine how the various parameters in Table 1 affect the time to transition.

There are several ways in which we can consider how the transition time to the quasi-self-similar "turbulent" state depends on the initial conditions. In this section, we will consider variations of the rms-amplitude (resulting in variation of $<\mathrm{ka}>$ in addition to $\langle\mathrm{a}>$ ), the effect of varying the short wavelength cutoff for a flat spectrum (variation of $\langle\mathrm{k}\rangle,\langle\mathrm{ka}\rangle$, and $\Delta \mathrm{k} /\langle\mathrm{k}\rangle$ ), the effect short wavelength "noise" on a long wavelength primary mode (variation of spectral shape and $\langle\mathrm{k}\rangle_{\mathrm{s}} \mid\langle\mathrm{k}\rangle_{1}$ ), and the effect of a long wavelength mode on a short wavelength spectral component (variation of $\left.\langle a\rangle_{s} /\langle a\rangle_{1}\right)$.

\section{Initial rms-amplitude and nonlinearity thresholds}

In order to quantify the dependence of the transition time on the initial rmsamplitude, three simulations were run in which the peak of the narrow gaussian spectrum (called $\mathrm{a}_{40}$ ) was varied over three orders of magnitude. The peaks of the initial spectra considered were at $\mathrm{a}_{40}=0.001 \mu \mathrm{m}, \mathrm{a}_{40}=0.01 \mu \mathrm{m}$, and at the nominal value of $\mathrm{a}_{40}=0.1 \mu \mathrm{m}$. This corresponds to an initial degree of linearity, expressed as $\mathrm{a}_{40} / \lambda_{40}$, of 2e-4. 2e-3. and 2e-2, respectively. In each case, the rms-amplitude was about 2.5 times greater than then $\mathrm{a}_{40}$.

Results from the calculations are shown in Fig. 19. Regardless of the initial amplitude, spike interaction begins when $\mathrm{h} / \lambda_{0} \approx 2$. This nonlinearity threshold for spike interaction is slightly greater with smaller initial nonlinearity $h_{0} / \lambda_{0}$. This could be due to the increased shock-deposited vorticity (RM) or increased instability Mach 
Spectrum peak (narrow gaussian)

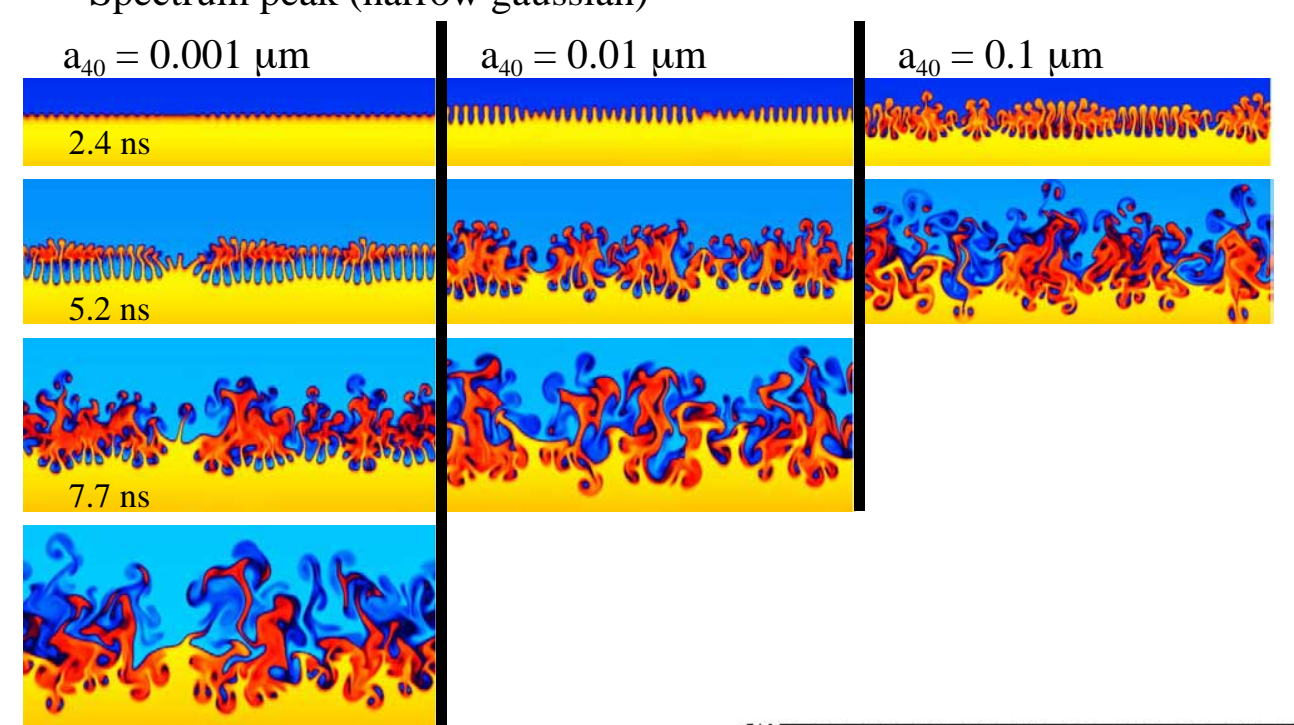

(a)
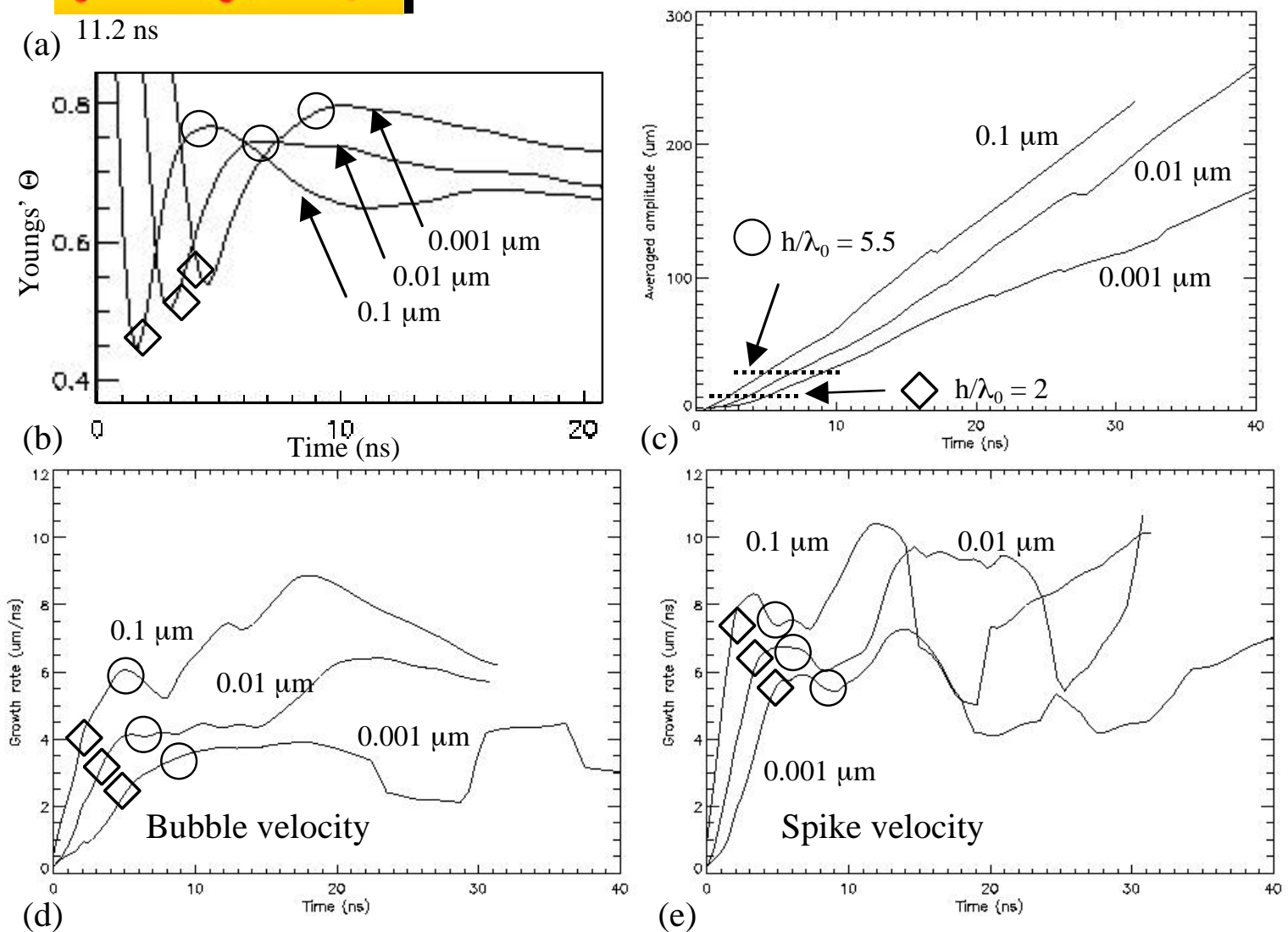

Figure 19: Dependence of amplitude growth and transition time on the initial $\mathrm{a}_{\mathrm{rms}}$ : (a) Log density plots show spike interaction begins when $h / \lambda_{0} \approx 2$. (b) The mixing parameter peaks at later times for smaller initial $\mathrm{a}_{\mathrm{rms}}$, coincident with the loss of initial transverse spectral information. (c) Amplitude histories show transition to the quasiself-similar regime when $\mathrm{h} / \boldsymbol{\lambda}_{0} \approx 5-6$. (d) Bubble and (e) spike velocity histories show that spike breakup results in a reduction in the growth rate followed by accelerated growth after establishment of the quasi-self-similar regime and the generation of larger scales. In (b)-(e), the spike interaction threshold $\mathrm{h} / \lambda_{0} \approx 2$ is denoted by diamonds and the transition threshold $\mathrm{h} / \lambda_{0}=5.5$ is denoted by circles. 
number in the larger-amplitude cases, or a combination of the two. At the same time spike interaction begins, the spike growth rate begins to saturate and the acceleration

of the bubble front begins to decrease. Spike breakup continues until $h / \lambda_{0} \approx 5-6$ (again somewhat greater for perturbations initially more linear), at which point the mixing parameter reaches its maximum value and the transverse density fluctuation spectrum has reached its asymptotic form. This signifies the loss of initial transverse spectral information and the emergence of the quasi-self-similar regime. At the same time, the post-transition amplitudes and velocity is strongly dependent on the initial rms amplitude [see Figs. 19(d)-19(e)]. Spike and bubble velocities subsequently increase again as the inverse cascade to larger scales progresses. The same sequence is followed for broader initial spectra, without significant change in the nonlinearity thresholds for spike interaction and transition.

\section{Effect of short-wavelength mode on large-scale interface structure}

We are interested in the effect of short wavelength modes on the global instability development for three primary reasons. First of all, some RT-unstable interfaces in real systems [possibly including the $\mathrm{Si} /(\mathrm{C}+\mathrm{O})$ interface in core-collapse supernovae $]^{44}$ are characterized by distinct long and short-wavelength spectral components. In order to accurately describe the instability development in such systems, we must first understand the importance of the short-wavelength modes. 
Secondly, simulations typically use initial spectra that do not extend all the way down to the viscous cutoffs. If unresolved scales have a significant impact on the large-scale interface structure, then such simulations are inadequate.

Finally, laser-driven instability experiments designed to study supernova hydrodynamics are often limited in modal content, typically to no more than a few prescribed modes plus small-scale noise. Since supernovae are presumably not so limited, the experiments are not truly representative of their astrophysical counterparts if short wavelength modes are important. In both cases, one system (a simulation or a laboratory experiment) is employed to study a second physical system that may be less limited in modal content, and it is important to understand the effect of the unresolved scales.

In this section, we consider the effect of short wavelength modes on the global instability development in two ways. First, we vary the short wavelength cutoff in a series of five simulations with initially flat spectra and observe the resulting variation in growth rates and interface structure. The long wavelength cutoff is in each case mode 4 , while the short wavelength cutoffs included in the study are $m_{\max }=\{80,40$, $20,10,4\}\left(\mathrm{m}_{\max }=\mathrm{m}_{\min }=4\right.$ for the single mode calculation $)$. This gives initial characteristic mode numbers of $\langle\mathrm{m}\rangle=\{42,22,12,7,4\}$, and relative spectral widths of $\Delta \mathrm{m} /\langle\mathrm{m}\rangle \approx\{1.8,1.6,1.3,0.9,0.0\}$. The initial rms-amplitude is set to $0.25 \mu \mathrm{m}$ in each case, giving linearity parameters $\langle\mathrm{a} / \lambda\rangle \approx\{0.006,0.005,0.004,0.003,0.005\}$.

Log density plots at several times are shown in Fig. 20(a) from all but the single mode simulation. There is more mixing early on when the initial spectrum extends to higher mode numbers [see Fig. 20(b)], and the inverse cascade to larger 

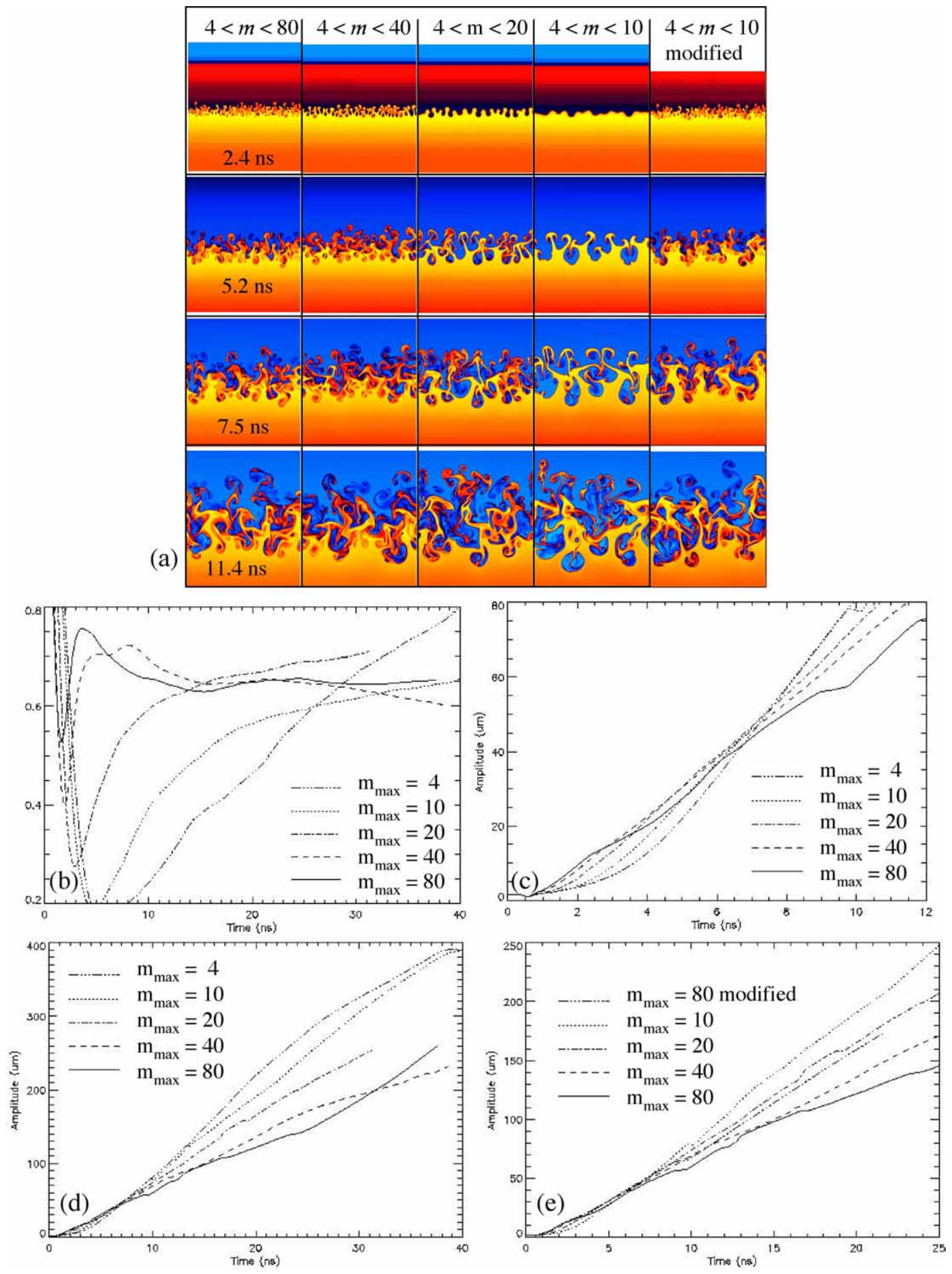

Figure 20: Variation of short-wavelength cutoff for flat spectrum from $\mathrm{m}_{\max }=4$ (single mode) to $\mathrm{m}_{\max }=80$. (a) Log density plots, (b) mixing parameter, and (c)-(d) amplitude histories show decreased mixing and enhanced growth with lower cutoff when the initial rms amplitude is fixed at $0.25 \mu \mathrm{m}$. (e) A modified $\mathrm{m}_{\max }=80$ is constructed in which the the first 7 modes are replaced with the spectrum from the $\mathrm{m}_{\max }=10$ calculation. The initial $\mathrm{rms}$ amplitude is increased by nearly $\sqrt{2}$ relative to the $\mathrm{m}_{\max }=10$, but the amplitude is reduced to below the $\mathrm{m}_{\max }=20$ result. 
scales proceeds more rapidly. Amplitude history plots show that higher shortwavelength cutoff leads to faster growth during the first few ns but reduced growth at later times [see Figs. 20(c)-20(d)]. The one exception to this pattern is the $\mathrm{m}_{\max }=80$ case, which ends up growing faster asymptotically than the $\mathrm{m}_{\max }=40$ case due to the emergence of a large single dense spike at about $25 \mathrm{~ns}$.

These observations can be partially explained as follows: Spectra extended to higher mode numbers initially give faster growth because of increased shockdeposited vorticity (RM) and because the RT exponential growth rate $\gamma \propto \sqrt{k}$ in the linear regime. The dominance of high modes ends when the low modes become nonlinear and their velocities begin to saturate at their terminal values. If there were no low-1 modes in the initial spectrum, or if their initial amplitudes were sufficiently small, then the growth of low-1 modes would be dominated by nonlinear interaction between high-1 modes (mode coupling). ${ }^{30}$ In our case, the initial amplitudes of the low-1 modes are of order $\mathrm{a} / \lambda \sim 0.05 / \sqrt{ } \mathrm{N}$ where the number of modes $\mathrm{N}$ varies from 1 to 81 . When $\mathrm{N}$ is less than or of order 10 , the preexisting low-1 modes become nonlinear within a few ns and mode coupling does not play a significant role. This is evident in the $\mathrm{m}_{\min }=10$ and $\mathrm{m}_{\min }=20 \log$ density time series, in which it is apparent that the large wavelength structure at $11.4 \mathrm{~ns}$ is correlated with that at $2.4 \mathrm{~ns}$. The difference in the late time growth rates is partially a reflection of the initial amplitudes of the low-l modes that begin to dominate the growth early on. These amplitudes are decreased when we increase the relative spectral width $\Delta \mathrm{k} /\langle\mathrm{k}\rangle$ while holding the initial rms amplitude constant. 
In addition to larger initial amplitudes in the long-wavelength modes, spectra with lower high-mode cutoff give faster growth because they transition to turbulence later. Consequently, they experience less of the density-gradient stabilization associated with enhanced mixing in the post-transition state. A modified $\mathrm{m}_{\max }=80$ interface was constructed to isolate the effect of enhanced mixing from the initialamplitude effect. In the modified initial spectrum, the first 7 modes in the $\mathrm{m}_{\max }=80$ spectrum were simply replaced with the spectrum from the $\mathrm{m}_{\max }=10$ case. Because of this change, the initial rms amplitude is just under $\sqrt{ } 2$ times greater than in the other cases, and the low l-mode amplitudes are the same as in $\mathrm{m}_{\max }=10$ case. Despite the increase in rms amplitude and a large increase in the number of unstable modes, the post-transition growth of the mix region is decreased rather than increased relative to the $\mathrm{m}_{\max }=10$ calculation [see Fig. 20(e)]. In fact, the resulting late-time amplitude history lies below the $\mathrm{m}_{\max }=20$ curve and only rises above the $\mathrm{m}_{\max }=40$ curve at about $12 \mathrm{~ns}$. Thus the presence of the short wavelength modes leads to a significant reduction in the nonlinear growth of the mixing layer.

The opposite effect was found by Milovich et al. in simulations of NIF double-shell ignition target designs ${ }^{45}$ In the double shell targets, instabilities develop on a metal/foam interface during capsule implosion. The perturbation spectrum was taken from measurements of an Omega glass capsule, and a series of calculations was run in which the number of modes was increased from about 40 up to several hundred. The angular resolution was determined such that the shortest-wavelength mode in the initial conditions was resolved to at least $20 \mathrm{ppw}$, and $1_{\min }$ was set to $12 \mathrm{in}$ each case. The late-time perturbation growth was found to increase with increasing 
$1_{\max }$, with a particularly dramatic increase when $1_{\max }$ was increased from 102 to 204 .

There are several differences between their system and ours that might contribute to this discrepancy. First of all, the number of modes is varied much more widely in their calculations than in our, and their initial spectrum falls off relatively slowly above mode 50. Since they do not renormalize the initial spectrum each time, runs with more modes have greater initial rms amplitudes. Secondly, modes in their initial spectrum are typically far more linear than ours. This allows more time for shortwavelength modes to couple and generate larger scales during the weakly nonlinear regime before saturation. The most significant difference, however, is that their low-1 modes do not have time to grow from the initial conditions up to nonlinear amplitudes. With $1_{\max }=54$, the perturbations at ignition time (the end point of the calculation) remain linear. Even with $1_{\max }=102$, the low-1 modes appear to have attained a degree of nonlinearity $\mathrm{a} / \lambda \sim 1$. When $1_{\max }$ is increased to 204 , however, the late-time perturbations are very nonlinear and scales larger than the initial conditions have been generated. This indicates that significant mode coupling and associated pumping of large scales to nonlinear amplitudes is possible only with $1_{\max }>200$. In our system, the low-l modes do not have to rely on mode coupling in order to reach large amplitudes on the time-scale of the experiment. Since the addition of shorter wavelengths does not increase the initial rms amplitude by more than a factor of $\sqrt{ } 2$, its main effect is to hasten the transition to a state with lower effective Atwood number and greater energy isotropy.

An extensive series of calculations was run to investigate the effect of short wavelength modes on the evolution of a single long wavelength mode. The long 
wavelength mode is mode 4 , with nominal initial amplitude of $2.5 \mu \mathrm{m}$. The shortwavelength component is bounded by modes 20 and 80, and typically has an rmsamplitude that is $1 / 10$ that of the nominal mode 4 amplitude. The shape of the shortwavelength spectral component was either narrow gaussian, broad gaussian, hyperbolic (1/k), or $1 / \mathrm{k}^{2}$ [see Fig. 4(a)]. A representative initial interface profile (with a 1/k short wavelength component) is shown in Fig. 4(e). Changes in spectral shape result in small changes in the characteristic mode number of the short wavelength component, and hence in the k-space separation parameter $\langle\mathrm{k}\rangle_{\mathrm{s}} \mid\langle\mathrm{k}\rangle_{1}$, which varies over 9.25-11.30. These simulations make up the upper family of amplitude history curves in Figs. 5(a) and 7(a) and the lower family of mixing parameter curves in Fig. 14. The single-mode amplitude history (no short-wavelength component) is the uppermost curve in the large-amplitude family, indicating that the short-wavelength component reduces the late-time perturbation growth by as much as $20 \%$. Plots of separate spike and bubble amplitude histories (not included) show that there is twice as much variation (about 30\%) in the spike amplitudes as there is in the bubble amplitudes (about 15\%).

We found in section IV.A that the location of a given curve within the smallamplitude family of Figs. 5 and 7 is not well correlated with the spectral shape. The same is true for the bi-component upper family. Figure 21 includes amplitude history plots from the single mode calculation, mode 4 plus hyperbolic shorts (with $\langle\mathrm{k}\rangle_{\mathrm{s}} \mid\langle\mathrm{k}\rangle_{1}$ $=10.75)$, and mode 4 plus broad gaussian shorts (with $\left.\langle\mathrm{k}\rangle_{\mathrm{s}} \mid\langle\mathrm{k}\rangle_{1}=11.30\right)$. The difference in amplitude between the two mode-4-plus-shorts cases is characteristic of the differences seen between different short-wavelength spectral shapes or different 
randomized amplitude and phase realizations of the same spectral shape. Again, the shape of the initial spectrum is not important in determining the late-time large-scale interface structure.

The principle effects of short wavelength modes on the large-scale interface structure are to increase the degree of mixing and accelerate the transition to the quasi-self-similar "turbulent" state [see Fig. 21(a)]. Both of these effects involve the interaction and breakup of spikes and an associated significant reduction in the overall growth rate of the spike front [see Fig. 21(b)]. The single mode spikes eventually break down as well under the influence of a numerical noise that effectively adds a short wavelength component. This happens later in time though, indicating that the rms amplitude of the short-wavelength is important in determining its effect on the long-wavelength mode. The late-time growth rate of the bubble front is increased if the breakup of spikes allows for bubble competition and merger on what would otherwise be a stable periodic array of bubbles [see Fig. 21(c)]. Because of the strong influence of the short-wavelength component on the transition time, coupled with the strong effect of transition on the global characteristics of the flow, systems comprised of a single mode or a few commensurate modes make poor surrogates for real physical systems. For broadband spectra, it appears that the presence and rms amplitude of the short wavelength component but not its spectral shape are important. This suggests that computational or experimental surrogates for systems dominated early on by long-wavelength modes need not accurately reproduce the details of the short-wavelength spectral component as long as the low l-modes are well resolved. Because this scenario might depend on the dimension of the 

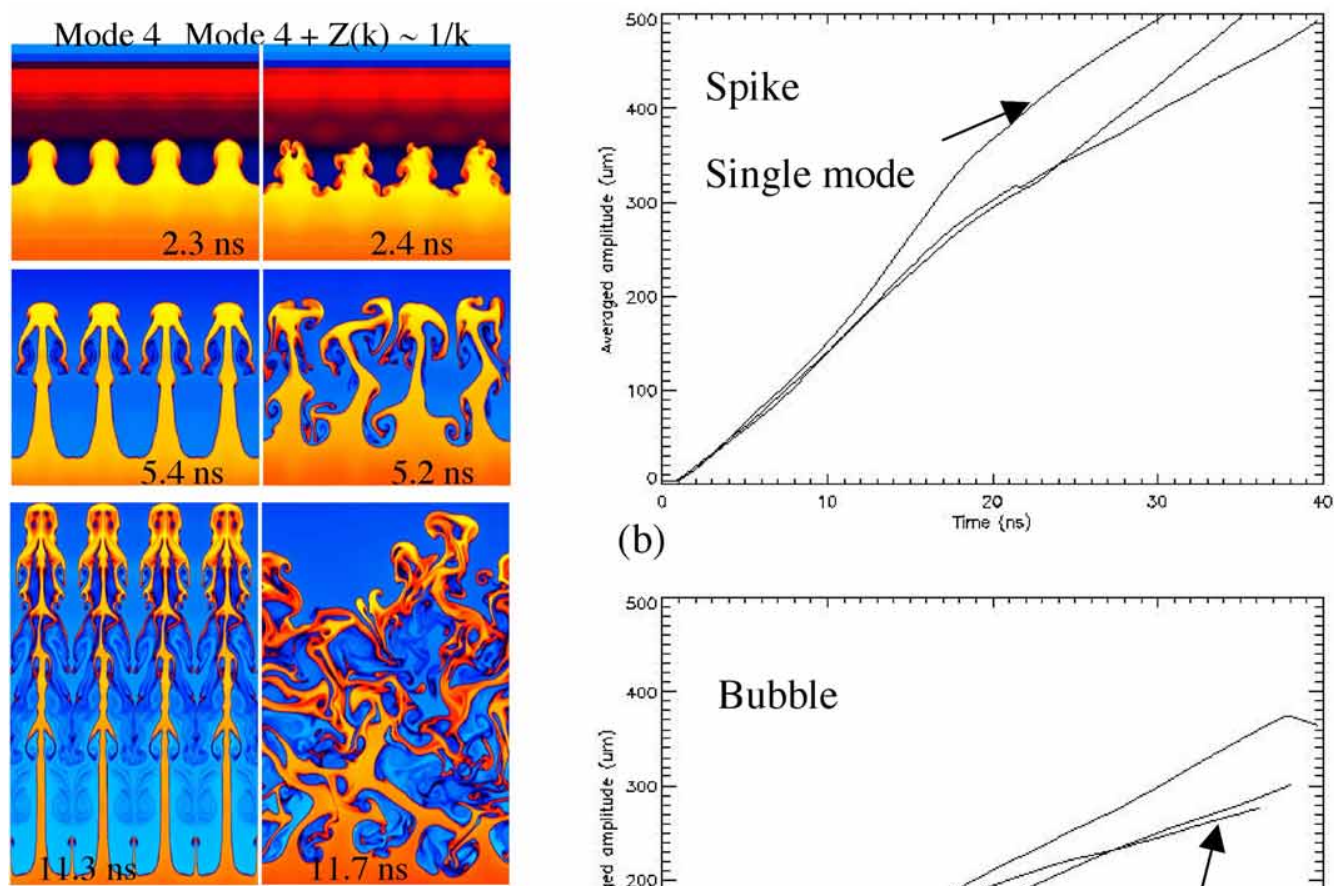

(b)

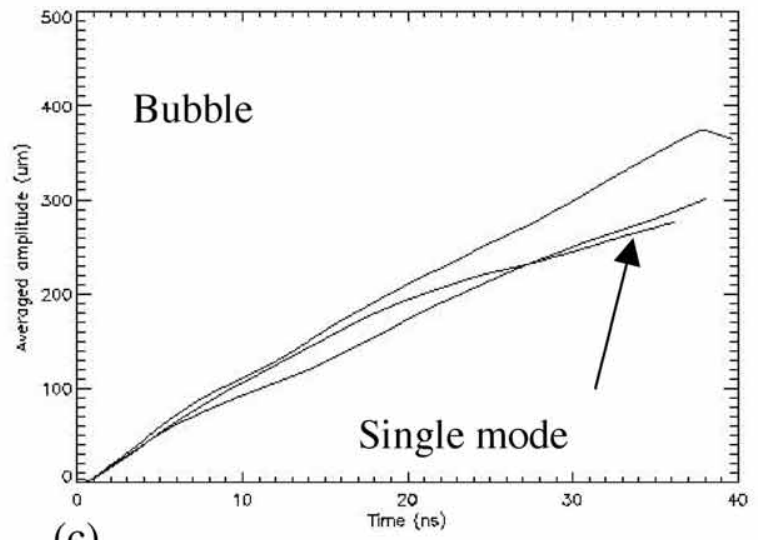

(c)

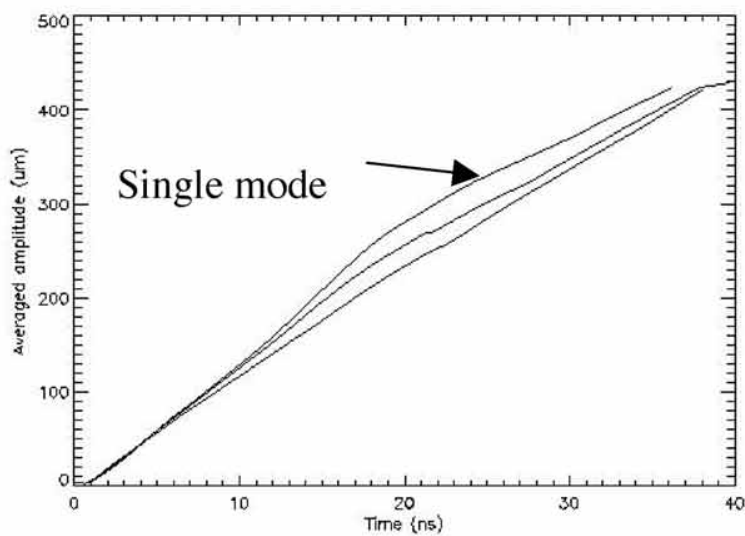

(d)

Figure 21: Effect of shorts on long: (a) Breaks the single-mode symmetry, resulting in (b) reduction in late-time spike growth and (b) enhancement of the late-time bubble velocity. (d) The net effect is an increase in the growth of the averaged amplitude. Amplitude plots include single mode 4 , mode 4 with hyperbolic shorts, and mode 4 with broad gaussian shorts. 


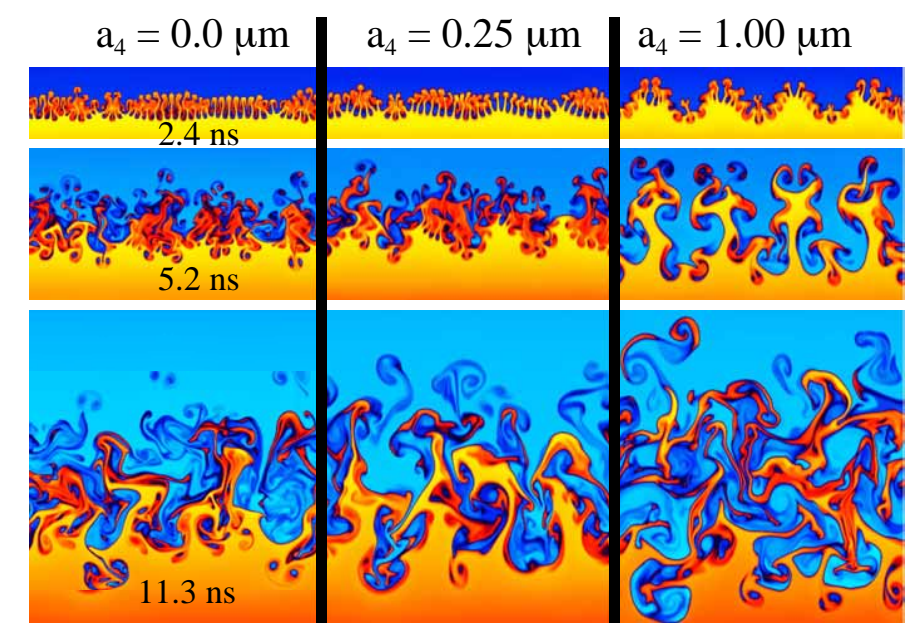

(a)

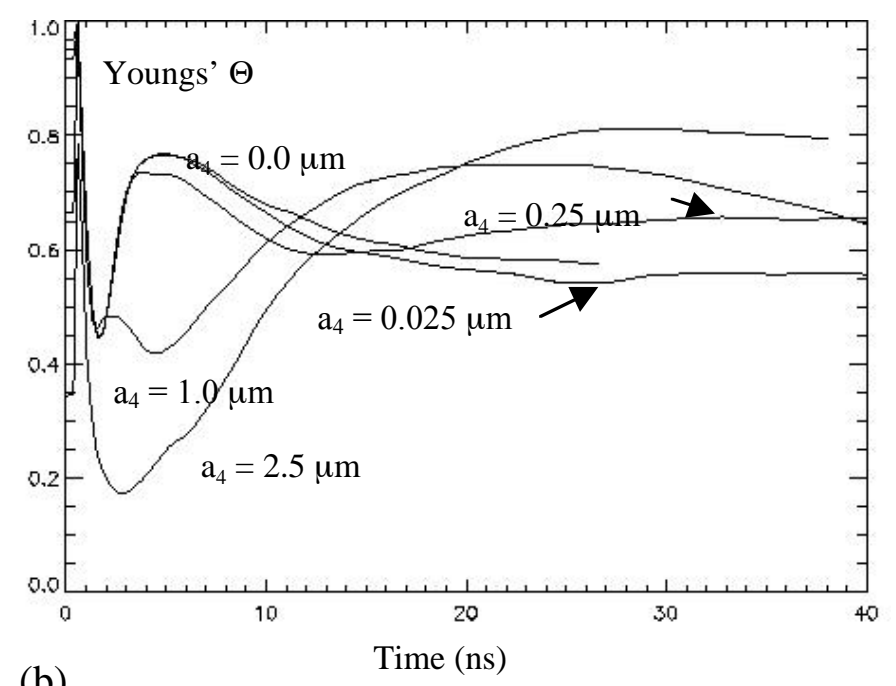

(b)

Figure 22: Effect of long on shorts. (a) Suppresses early-time growth of small-scale structure and (b) delays the transition to "turbulence".

perturbation, we will consider in a later paper the effect of unresolved modes on the evolution of 3D systems capable of undergoing a turbulent mixing transition.

\section{Variation of $\left\langle a_{s}\right\rangle \mid\left\langle a_{1}\right\rangle$}

Finally, we consider the variations in the relative rms amplitudes of the long and short-wavelength components. We ran a series of five calculations, each of which included the same narrow gaussian short-wavelength spectrum plus mode 4 (see Fig. 
22). The relative amplitude parameter $\langle a\rangle_{s} \mid\langle a\rangle_{1}$ was controlled by varying the mode 4 amplitude from 0.0 to $2.5 \mu \mathrm{m}$, with intermediate values of $0.025,0.25$, and $1.0 \mu \mathrm{m}$. When the initial amplitude of mode 4 is less than or equal to the rms amplitude of the short-wavelength component $\left(\langle a\rangle_{s}|<a\rangle_{1} \geq 1\right)$, the instability evolution closely resembles the shorts-only case. The transition time in particular is not sensitive to the presence or amplitude of mode 4 as long as its amplitude is small [see Fig. 22(b)]. When the mode- 4 amplitude is larger than $\langle a\rangle_{s}$, there is a qualitative change in the instability development. Mode 4 begins to dominate the growth within a few ns, in a time that is roughly equal to or less than the time required for the short-wavelength modes to reach their nonlinearity thresholds and undergo transition. Shear that develops along the mode- 4 spikes and bubble as they grow into the nonlinear regime greatly inhibits the development of small-scale structure and delays the time to transition [see Fig. 22(a)]. This delay contradicts what one would expect based on transition to shear-layer turbulence, which should appear earlier along larger spikes due to their faster terminal velocities and consequently higher Reynolds numbers. The same stabilizing effect was noted and described by Ofer $e t$ al. in their discussion of the effect of a secondary long-wavelength mode on a short-wavelength primary mode ${ }^{46}$, and is also visible in a calculation by Youngs. ${ }^{5}$ The short-wavelength spectral component is in large part lost and must be regenerated later after the mode-4 spikes reach their interaction and transition nonlinear thresholds.

\section{Comparison with classical case}


Some of the results we have found in our 2D, high-resolution simulations of blast-wave-driven systems are applicable to classical RT systems, while others are not. Potential sources of differences are the time-dependence of the drive and compressibility. Compressibility effects include the RM contribution, moderately high instability Mach number (up to $\mathrm{M}^{2} \sim 0.1$ in our calculations), stretching of perturbations due to material decompression, and the associated density gradient behind the shock front.

Our "effective box size", which arises because of the decay of the driving acceleration, is absent in classical systems. However, most if not all multi-mergergeneration experiments and simulations performed to date are affected by a physical or computational box size at late times.

The process of spike interaction and breakup will likely proceed similarly in both systems. However, we might expect to find lower nonlinearity thresholds in our case due to shock-deposited vorticity and higher instability Mach number. From classical RT simulations of Ofer et al., it appears that spike interaction begins at about $\mathrm{h} / \lambda \sim 2-3 .{ }^{46}$ This might be slightly higher than our value of $\mathrm{h} / \lambda \sim 2$, but the difference seems too small to be meaningful.

In planar blast-wave-driven systems, a true self-similar regime independent of initial conditions is not possible due to decompression. We have found in its place a quasi-self-similar regime that is limited in time and transverse scale due to drive decay. Self-similarity in classical RT systems remains a possibility (but even there has not yet been conclusively demonstrated), and we might expect a correspondence between transition requirements in classical systems and the onset of quasi-self- 
similar growth in blast-wave-driven systems. Early rocket-rig experiments ${ }^{6}$ and simulations ${ }^{5}$ suggested self-similar-like growth after about 2.5 generations of bubble merger. This is frequently expressed as the requirement that transverse scales ten times larger than the most unstable mode or the initial characteristic scale must be generated in order to transition to take place. More recent water-channel experiments are also consistent with this requirement. ${ }^{47}$ After 2.5 merger generations, the initial characteristic mode 40 in our shorts-only calculations has shifted to mode 7. According to Fig. 8, this occurs at about 6 ns. The observed transition time, based on the mixing parameter or the loss of transverse spectral information, is at about 3-4 ns or after just one merger generation. Again, this accelerated transition is likely due to enhanced vorticity due to RM and very fast spike growth, both of which might facilitate spike interaction and breakdown. In 2D calculations with instability Mach number of the same order as ours (but without a shock), Glimm et al. report spike interaction and transition to a "multiply connected structure" after about 1.5 merger generations. ${ }^{48}$ The fact that they consider this transition (and the resulting growth rate reduction) to be an unphysical $2 \mathrm{D}$ artifact points to the need for comparison with highly-resolved 3D calculations.

Despite the decreased drag on 3D objects and the suggestion that the posttransition growth reduction in $2 \mathrm{D}$ calculations is artificial, early 3D classical RT calculations by Youngs show reduced growth relative to the $2 \mathrm{D}$ case in the turbulent regime.$^{49}$ This reduction is likely due to increased dissipation in $3 \mathrm{D}$, as suggested by Youngs in the same paper. A similar effect has been observed in ongoing state-of-theart simulations by Cook et al., who consider the instability growth in terms of four 
evolutionary stages. ${ }^{41}$ After a short period of independent modal growth, a modemode interaction phase begins when $\mathrm{h} /\langle\lambda\rangle \sim 2$ (equal to our threshold for spike interaction). During this "weak turbulence" phase, the mixing parameter reaches a local maximum at $\mathrm{h} /\langle\lambda\rangle=4.3$ ( similar to our peak at $\mathrm{h} /\langle\lambda\rangle \sim 5$ ). A second increase in the mixing parameter occurs between $\mathrm{h} /\langle\lambda\rangle \sim 8-19$, which they call the turbulent mixing transition. The mixing and similarity parameters appear to have reached their asymptotic values by about $\mathrm{h} /\langle\lambda\rangle=19$, which marks the beginning of a strong turbulence phase that extends to the end of their calculation at $h /\langle\lambda\rangle=30$. In some of our 2D calculations, we too see a second peak in the mixing parameter [see Fig. 19(b) for an example] approached over about $h /\langle\lambda\rangle \sim 14-24$. We find these parallels encouraging in light of the high-resolution, high-order nature of the Cook et al. calculations along with our expectation that transition should proceed qualitatively similarly in classical and blast-wave-driven systems. More detailed comparisons, however, will have to await completion of our 3D blast-wave-driven simulations.

\section{$\underline{\text { VI. Conclusions }}$}

We have presented and discussed results from a series of over $702 \mathrm{D}$ highresolution AMR simulations of hydrodynamically-unstable interfaces driven by a strong blast wave under NIF-like drive conditions. The mix-width time histories show no apparent approach to a self-similar regime independent of the initial conditions. This is due to decompression and drive decay, which result in an asymptotic velocity 
that depends on the amplitude and time as well as on the transverse scale and the degree of mix in the layer. For sufficiently long but finite experiment duration, drive decay also leads to an effective box size that sets a maximum transverse scale that can be generated. After this scale is reached, the inverse cascade is halted and the growth is no longer self-similar-like. There is, however, a period of quasi-self-similar growth after generation of scales larger than the initial conditions but before the generation of the effective-box-size scale.

The existence of the quasi-self-similar state and the drive-imposed effective box size make the blast-wave-driven case distinct from classical RT. However, transition to the quasi-self-similar state is very similar to its classical counterpart. In both cases, transition is marked by an increase in the degree of mixedness, a decrease in the spike velocity, and often an increase in the bubble velocity.

For continuous and bi-component (short on long) spectra, we have identified several parameters that classify and characterize the initial conditions. We have investigated how variations of a subset of these parameters can affect the observable properties of the deep nonlinear instability evolution. We found, for example, that a long wavelength mode can inhibit the development of small scales and delay the transition to a turbulent-like state when its amplitude is larger than the rms amplitude of the short-wavelength spectral component.

Most notably, apparently random variations observed in late-time amplitudes and growth rates were not well correlated with initial spectral shape. The presence of the short wavelength component is important for facilitating the transition to turbulent-like flow, but its effect on the large scales does not depend strongly on its 
spectral shape. Only the average properties are important, such as the initial rms amplitude and characteristic wavenumber. This bodes well for simulations of similarly strongly-driven systems that leave a portion of the short-wavelength end of the spectrum unresolved. As long as the system contains some fast-growing and interacting modes that can be resolved computationally or reproduced experimentally (and has the correct initial rms amplitude), the late-time instability evolution will likely closely resemble the fully resolved or complete system. This reaffirms the hope that laser-driven experiments can serve as useful and relevant platforms for studying compressible mixing in supernovae despite their drastically more limited available range of scales. Similarly, carefully-designed numerical simulations need not necessarily reproduce the full range of spectral details present in their physical counterparts in order to reasonably reproduce the late-time large-scale interface structure. These conclusions apply in particular to systems with long-wavelength modes large enough in amplitude to reach the nonlinear phase early on.

It is important to remember that these conclusions are based solely on $2 \mathrm{D}$ calculations and might be altered somewhat in 3D. In a later paper, we will extend the discussion and analysis to high-resolution 3D simulations currently underway. This analysis will include a discussion of the effects of initial conditions on the turbulent mixing transition and the nature of the subsequent turbulent flow. 


\section{Chapter 6: Transition to Turbulence and Effect of Initial Conditions on 3D Compressible Mixing in Planar Blast- wave-driven Systems}

\section{Introduction}

An interface between two fluids becomes hydrodynamically unstable when it transmits a blast wave down the density gradient. Perturbations grow under the combined influence of the Rayleigh-Taylor ${ }^{1,2}(\mathrm{RT})$ and Richtmyer-Meshkov ${ }^{3,4}(\mathrm{RM})$ instabilities. In addition, material decompression behind the shock front results in amplitude stretching that represents a significant contribution to the growth at late times. ${ }^{5}$ RM dominates at very early times, but its contribution relative to RT quickly becomes insignificant as the shock-deposited vorticity spreads out and decays away., In the nonlinear phase, initial perturbations evolve into spikes of denser material growing in the direction of shock propagation and bubbles of less dense material lagging ever further behind the unperturbed interface position. If the interface is driven by a sufficiently strong blast wave, 2D and/or 3D secondary instabilities will eventually cause nonlinear spikes to interact with one another and break down. Energy and momentum are thereby diverted into the transverse direction and a transition to a turbulent or turbulent-like state occurs. 
In a previous paper, we considered the effect of the initial perturbation spectrum on the nonlinear evolution of a 2D blast-wave-driven system, including spike interaction. ${ }^{6}$ Based on high-resolution 2D Raptor ${ }^{7}$ simulations, we concluded that memory of certain aspects of the initial conditions, such as the rms amplitude, is retained in the mix-width at all times. Spike interaction and breakup was found to correspond to an increase in small-scale mixing, a significant reduction in spike velocity, and a loss of initial transverse spectral information. Consequently, we referred to it as a 2D turbulent mixing transition. At late times, the inverse cascade driven by bubble competition and merger was halted due to drive decay, which imposed an effective box size on the system. After transition but before the emergence of the effective box size, we observed a quasi-self-similar regime during which the similarity parameter $\langle\lambda\rangle / \mathrm{h}$ decays slowly in time, with a value only weakly dependent on the initial conditions.

In this paper, we extend this study to include 3D calculations of systems that are otherwise identical to the laser-driven targets described in the 2D paper. Our goal is to determine how the deep nonlinear instability evolution differs in the 3D case, where vortex stretching makes transition to full 3D turbulence possible. In particular, we would like to understand how the initial conditions affect the time to transition and the nature of subsequent turbulent flow.

We will show that nonlinearity thresholds for spike interaction and breakup are not significantly changed in $3 \mathrm{D}$, and that the post-transition state is more thoroughly mixed than in the 2D case. More mix gives a lower effective Atwood number that leads to a large reduction in the bubble growth rate relative to the $2 \mathrm{D}$ 
values despite the decreased drag in 3D. Surprisingly, the velocity of the spike front is not significantly changed when going from 2D to 3D. It is however reduced relative to its pre-transition value. This is particularly interesting in light of the fact that $2 \mathrm{D}$ simulations of blast-wave-driven mixing in core-collapse supernovae consistently underpredict the asymptotic spike velocities by about a factor of two (see Ref. 8 and references therein). This problem is not solved by $3 \mathrm{D}$ calculations that predict spike velocities the same as in $2 \mathrm{D}$, suggesting a balance between velocity enhancement due to decreased drag and reduction due to increased small-scale mixing.

\section{Transition to turbulence in RT-instability-driven systems}

Before continuing on with simulation results, we wish to clarify what we mean by turbulence. This is particularly important for turbulence in multimode RT systems because there are at least four ideas of turbulence that appear in the literature, and they do not all affect the instability growth in the same way.

First of all, RT-unstable systems can exhibit turbulence in the classical fluid mechanical sense. Unfortunately, there is no universal consensus on what classical turbulence is either. We describe it as a disordered state that undergoes random fluctuations in both space and time and is characterized by energy-flow from some large driving scale down to dissipative scales via the 3D phenomenon of vortex stretching. When driven by the RT instability, there is a range of driving scales set by 
the density spectrum or bubble size distribution. When bubble competition and merger are active, the dominant driving scale for the turbulence grows in time.

Transition to classical turbulence typically requires that the Reynolds number be greater than some threshold value. ${ }^{9}$ This requirement amounts to sufficient spectral separation between the driving and dissipative scales. Dimotakis observed that many systems exhibit a transition to a state of increased molecular mixing, which he called the turbulent mixing transition, above a critical Re of about $20,000 .^{10}$ This corresponds to three orders of magnitude separation between the driving and dissipative scales, allowing for an extended inertial range that is decoupled from both. Above the critical Re for the mixing transition, the internal structure of the flow is less Re-dependent than before the transition.

In non-stationary flows, there is a time constraint as well. ${ }^{11-14}$ Even if the Reynolds number is high enough that an inertial range can in principle exist, transition cannot take place until the inertial range has had time to develop. A final requirement for transition that is common to both non-stationary and stationary flows is the existence of some seed perturbation that instabilities can act on. The seed requirement is often not focused on because it will naturally be satisfied in virtually every system, whether physical or computational, due if nothing else to thermal or numerical noise.

The turbulent mixing transition often takes place in flows that have already undergone a transition from a laminar, ordered state to a disordered, spectrally complex state. ${ }^{10}$ The term "turbulence" is often also applied to any such system that appears random, regardless of whether or not there is a forward energy cascade via 
vortex stretching down an inertial range. This includes 3D simulations that leave the physical dissipation range and much of the inertial range unresolved. The resulting computational $\mathrm{Re}$ is often greatly reduced relative to the actual $\mathrm{Re}$ in the modeled system, even to the point that there is no discernible inertial range and the computational Re is lower than the experimentally observed critical Re. We will refer to such flows as weakly turbulent or turbulent-like, and to the initiating transition as the weak transition.

Two-dimensional turbulence is distinct from its 3D counterpart in that it lacks the vortex stretching and associated forward cascade that are central to 3D turbulence. There is, however, a forward enstrophy cascade and an inverse energy cascade driven by vortex pairing and merger. ${ }^{15,16}$ Since $2 \mathrm{D}$ simulations are often used to model 3D turbulent systems, it is important in each case to consider whether or not transition in the $2 \mathrm{D}$ system proceeds similarly to transition in the analogous fully resolved $3 \mathrm{D}$ system. This will likely depend on whether or not the latter is driven by instabilities that are inherently 3D (such as the Widnall vortex ring instability ${ }^{17}$ ). In such cases, 2D calculations of course cannot be counted on for predicting the transition time.

Finally, we note that the word turbulence is sometimes used to describe the inverse cascade, driven by bubble competition and merger in $3 \mathrm{D}$ as well as in $2 \mathrm{D}$, that is characteristic of nonlinear multimode RT evolution. This continual generation of successively larger scales leads to acceleration of the bubble front and explains the conjectured loss of initial conditions and establishment of a self-similar regime. ${ }^{18-20}$ Like transition to classical turbulence, transition to the self-similar regime requires time, space, and a seed. The seed spectrum must contain multiple, incommensurate 
modes that are unstable (ie not so small that they are stabilized by viscosity or other mechanisms). Otherwise, scales larger than those present in the initial conditions cannot be generated and the asymptotic state will be characterized by a stable, periodic array of bubbles. The characteristic wavelength of the spectrum will shift towards the low-l end of the initial spectrum, but will not pass beyond it. Noise (including numerical) present in the system can of course serve as the required seed, eventually triggering the interaction and breakup of spikes, but the transition will be delayed. ${ }^{6}$

The space requirement for transition to self-similar "turbulence" is that the system or box size be at least several times larger than the longest-wavelength significant modes present in the initial conditions. ${ }^{19}$ There must also be sufficient time for larger scale to be generated. If the space requirement is not met and until the time condition is satisfied, the scale-invariant bubble distribution ${ }^{21}$ will not be realized.

In blast-wave-driven systems (or other system with similarly decaying drive), the effective box size can play the same role as the physical box size in limiting the inverse cascade. ${ }^{6,22}$ If the drive becomes very small at late times but does not vanish, then thinking in terms of the effective box size amounts to a transformation of the time requirement into the spatial domain.

\section{Effect of transition on RT instability growth}


The evolution of an RT-unstable interface is likely to be similarly affected by transition to a classically turbulent or turbulent-like 2D or 3D state. Our previous 2D simulations agree with 2 and 3D simulations of others that such transitions leads to a higher degree of small-scale "molecular" mixing and typically a reduction of the growth rate. ${ }^{6}$ We have noted, however, that the velocity of the bubble front can be increased if the transition leads to bubble competition in what would otherwise be a stable, periodic array. In that case, transition to a classically turbulent or turbulentlike state provides the seed requirement for a subsequent transition to quasi-selfsimilar "turbulence". In our simulations, the 2D transition was found to occur when the dominant modes in the spectrum reached a nonlinearity threshold of about $h / \lambda \approx$ 5-6. For spectrally complex initial conditions, the 2D and self-similar transitions occurred virtually simultaneously. In single or few-mode systems, the self-similar transition sometimes occurred significantly later than the 2D transition.

While transition to a classically turbulent or turbulent-like state leads to enhanced mixing that tends to reduce the RT growth rates, ${ }^{23,24}$ transition to a (quasi)self-similar regime leads to an inverse cascade that tends to enhance the perturbation growth. ${ }^{25}$ When both transitions are coincident, the overall effect on the growth rates depends on which effect wins out. Simulations in 2 and 3D tend to show an initial growth rate reduction, especially for the spikes, but the growth must eventually be enhanced if the system allows for unlimited generation of larger structures with higher terminal velocities. ${ }^{6,24}$

We now consider the question of coincidence among the various transitions. It has been proposed that the mixing-transition time in RT-unstable systems is limited 
by the viscous growth of a shear layer along the spike stalks, and with it the Liepmann-Taylor scale that sets the low-l end of the inertial range. ${ }^{13,14}$ However, for broadband systems in a big box at high Reynolds number (ie seed and space requirements met for both classical and self-similar transitions), the mixing transition is preceded by nearly coincident weak and self-similar transitions. Once modes become nonlinear and begin to couple and generate larger scales, marking transition to self-similar turbulence, spikes soon reach their nonlinearity interaction and breakup thresholds. ${ }^{6}$ These interactions drive mix-layer-scale vortices, effectively shortcircuiting the process whereby the low-l end of the inertial range is limited by the viscous growth of a shear layer. Even in 2D, spike interaction and breakup due to Kelvin-Helmholtz (KH) activity gives an abrupt forward cascade, thereby temporarily mimicking full 3D turbulence characterized by vortex stretching and the associated transfer of energy down to the dissipative scales. Diffusive growth of the shear layer will likely provide the time limitation for transition to classical turbulence only in single-mode or otherwise spectrally simple systems.

When the mixing transition occurs in a system that has already undergone transitions to weak and self-similar turbulence, it can only cause a reduction of the growth rates by effectively lowering the Atwood number within the mix layer.

At low Re, the weak and self-similar transitions need not be coincident. For example, consider that the inverse cascade can occur at any Reynolds number, and in $2 \mathrm{D}$ as well as 3D. Equivalently, the presence of an inverse cascade does not imply strong interactions between spikes and the associated increase in mixedness. The Reynolds number will eventually become high if the inverse cascade is allowed to 
continue long enough $\left(\operatorname{Re} \sim \lambda^{3 / 2} \mathrm{~g}^{1 / 2}\right.$ if $\left.\mathrm{h} \sim \lambda\right)$, but the system can be "turbulent" in the self-similar sense at lower Re as well.

Conversely, a system with simple modal content can exhibit localized classical turbulence, including the mixing transition, if the Reynolds number is high enough. For example, in RM shock tube experiments by Jacobs et al. ${ }^{26}$ secondary instabilities lead to transition to classical turbulence within the $\mathrm{KH}$ rollups of singlemode spikes, while the large-scale single-mode structure remains intact and there is no bubble competition. Even so, spike interaction and breakup should increase the extent to which areas of turbulence permeate the mix region.

\section{$\underline{\text { IV. Effect of decompression and drive decay in classical turbulence }}$}

After an initial compression upon passage through he shock front, material in a blast-wave-driven system undergoes decompression in the post-shock velocity gradient. This expansion has an effect on any developing turbulence that can be understood by considering the vorticity equation for compressible fluids:

$$
\begin{aligned}
\frac{d \omega}{d t}=\omega \cdot \nabla \mathrm{v} & -\omega(\nabla \cdot \mathrm{v})+\frac{1}{\rho^{2}} \nabla \rho \times \nabla P+\nabla \times g+v \nabla^{2} \omega \\
& +\nabla v \times \nabla^{2} \mathrm{v}+\nabla \frac{1}{\rho} \times(\nabla \mathrm{v} \cdot \nabla \mu)+\frac{1}{\rho} \nabla \times(\nabla \mathrm{v} \cdot \nabla \mu)
\end{aligned}
$$

where $\mu$ and $v$ are, respectively, the dynamic and kinematic viscosities. If we neglect the baroclinic and viscous terms and assume only conservative forces, then this becomes 


$$
\frac{d \omega}{d t}=\omega \cdot \nabla v-\omega(\nabla \cdot v)
$$

Next, we make the reasonable assumption that decompression in the $\mathrm{u}_{\mathrm{z}} \approx \beta \mathrm{z} / \mathrm{t}$ rarefaction is the only significant deviation from incompressibility. In that case, we find

$$
\frac{d \omega}{d t}=\omega \cdot \nabla \mathrm{v}-\beta \frac{\omega}{t}
$$

The first term on the right-hand side describes vortex stretching, and the second represents the decay of vorticity due to the decompression. Finally, we expand v into the compressible part $\mathrm{u}_{\mathrm{z}}$ (which is also irrotational) and the incompressible part v', which, when inserted into (3), gives

$$
\frac{d \omega}{d t}=\omega \cdot \nabla \mathrm{v}^{\prime}+\hat{z} \beta \frac{\omega_{z}}{t}-\beta \frac{\omega}{t}=\omega \cdot \nabla \mathrm{v}^{\prime}-\beta \frac{\omega_{\perp}}{t},
$$

where $\omega_{\perp}$ is the transverse vorticity vector. We can draw two conclusions from Eq. (4) about the effect of the decompression on turbulence. First of all, the vortex stretching term does not include a contribution from the decompression. This is because any increase in vorticity due to stretching in the rarefaction is exactly balanced by the vorticity decay term. Thus the parallel (to the shock propagation direction) component of the vorticity is unaffected by the decompression. The transverse vorticity, on the other hand, remains "frozen into" the fluid and decays as the density decreases. If the turbulence was initially isotropic and viscosity not important, this should lead to an asymmetric vorticity field at late times biased in the parallel direction. In fact, vorticity due to shock deposition and RT strongly favors the transverse direction, with a significant parallel component arising only with the onset of strong spike interaction. Decompression may therefore help drive a post-transition 
vorticity field that is strongly anisotropic at the large scales towards isotropy by retarding the decay of its parallel component.

For a high Mach number Taylor-Sedov blast wave, we found in an earlier paper that the interface deceleration scales approximately as $g \sim \mathrm{t}^{-2[1-1 /(\gamma(\mathrm{d}+2))]}$ where $\gamma$ is the adiabatic index and $\mathrm{d}$ is equal to the dimension of the blast wave (ie three for spherical and one for planar). ${ }^{22}$ If we neglect for the moment the decompression and assume that the characteristic wavelength scales like $\left[\int \sqrt{g(t)} d t\right]^{2}$ as expected for incompressible RT in the scale-invariant regime, ${ }^{19,27}$ then we find that the Reynolds number scales like $\operatorname{Re} \sim \mathrm{t}^{1+2 /(\gamma(\mathrm{d}+2))}$, compared to $\mathrm{Re} \sim \mathrm{t}^{3}$ in the constant $\mathrm{g}$ case. Thus the Reynolds number continues to increase in the blast-wave-driven case, but it does so much more slowly than with constant acceleration. For a system driven by a real blast wave, the driving acceleration will eventually vanish and any turbulence will be left to decay away.

\section{Calculation setup and description}

The simulations are performed using the multi-physics radiation hydrodynamics code Raptor, which uses a $2^{\text {nd }}$ order (in space and time) Godunov method applied to the Euler equations. ${ }^{7}$ Raptor is parallelized and uses adaptive mesh refinement (AMR), making it well-suited to problems such as ours that require high resolution in only a portion of the computational domain. The calculation setup is nearly identical to our 2D simulations detailed elsewhere, ${ }^{6}$ and only an abbreviated 
description will be given here. The only difference in the 3D calculations is that the initial spectra are now typically 3D, and the nominal resolution is 256 cells across the computational domain instead of 512. The highest level of refinement is reserved for the interface region, and the total number of cells grows with the mix width, reaching (to date) over fifty million.

Our hypothetical target [see schematic in Fig. 1(a)] consists of a $150 \mu \mathrm{m}$ plastic pusher section (density $1.42 \mathrm{~g} / \mathrm{cc}$ ) in contact with a cryogenic hydrogen (density $0.086 \mathrm{~g} / \mathrm{cc}$ ) $2.2 \mathrm{~mm}$ payload section. An initial perturbation is imposed at the pusher/payload interface, and the width the computational domain was typically 200 $\mu \mathrm{m}$. Open boundary conditions are used in the parallel (to the shock) direction while periodic conditions are specified in the transverse direction.

The end of the pusher opposite the perturbation is driven with a $25 \mathrm{~kJ}, 1 \mathrm{~ns}$ laser pulse, which launches a strong planar blast wave into the target. Planar experiments with these drive properties will be possible within a few years as the National Ignition Facility ${ }^{28}$ (NIF) becomes operational. The simulations are initiated with a high-velocity, heated, compressed slab with characteristics taken from a laserdriven Lasne ${ }^{29}$ simulation at the end of the laser pulse. The Mach numbers of the incident and transmitted blast waves are in the range of 10-30, where the precise value depends on the degree to which x-ray preheat can be controlled (the incident Mach number with no preheat would be about 60). The resulting initial interface speed is about $130 \mu \mathrm{m} / \mathrm{ns}$ [see Fig. 1(b)]. The post-shock Atwood number remains nearly constant at about 0.7 .

In this paper, we will present results from four high-resolution (256 ppb) 


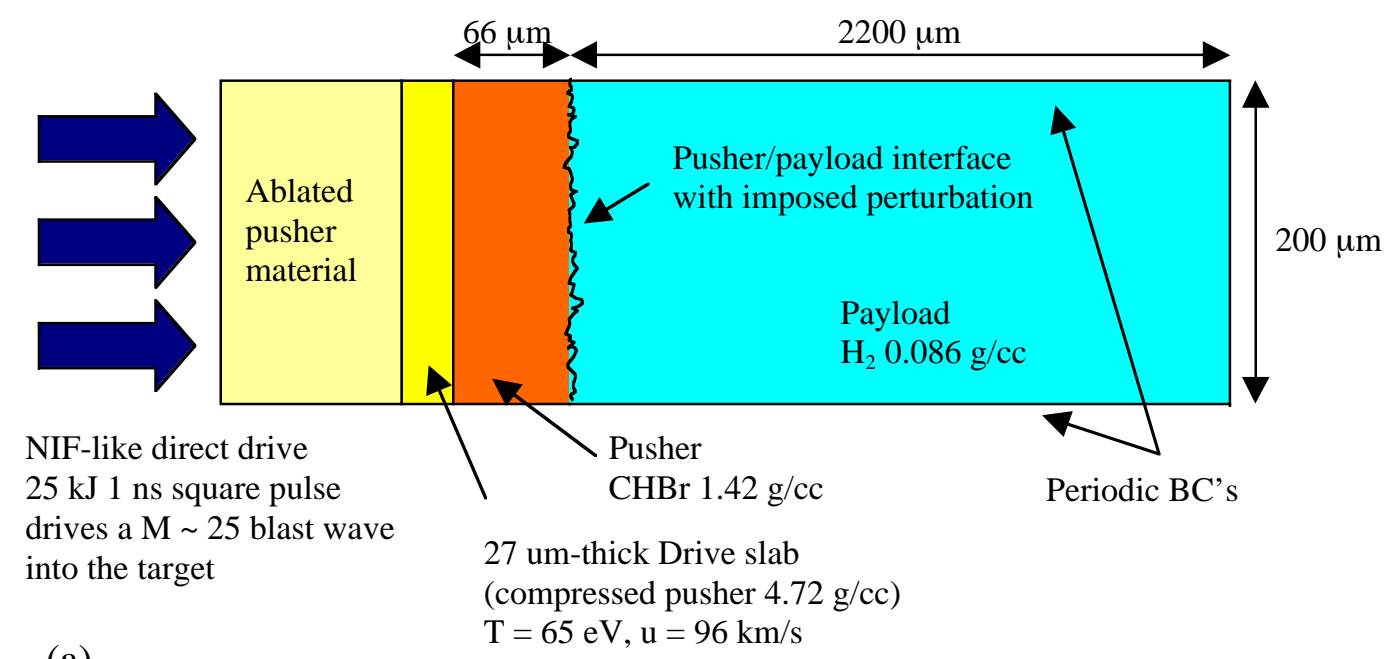

(a)

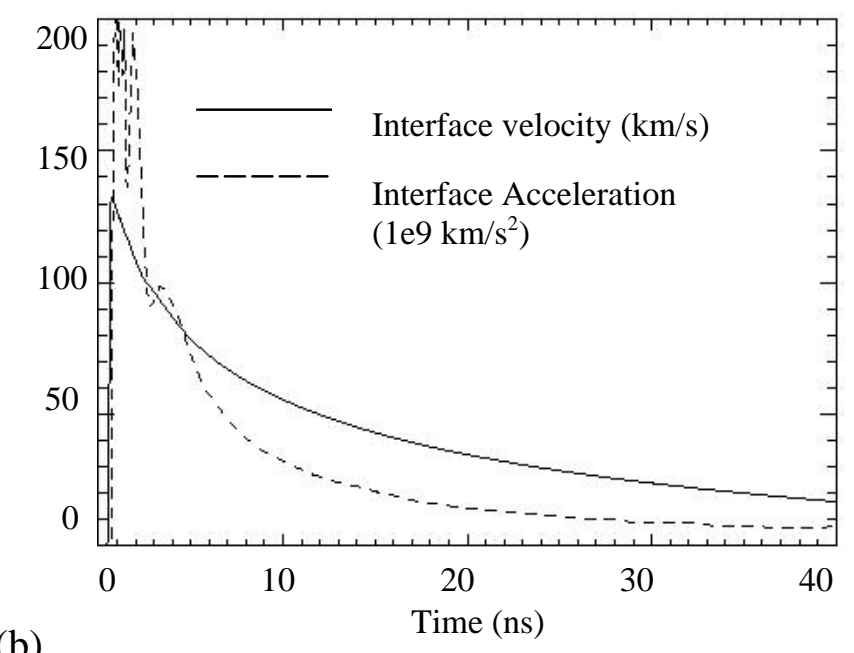

Figure 1: (a) Target schematic (not to scale). (b) Variation in time of interface velocity and deceleration.

multimode runs, one low-resolution $(128 \mathrm{ppb})$ mode 4 "egg-crate" run [given by $\mathrm{z}_{0}=$ $(2.5 \mu \mathrm{m}) \cos \left(\mathrm{k}_{4} \mathrm{x}\right) \cos \left(\mathrm{k}_{4} \mathrm{y}\right)$, where $\mathrm{k}_{4}$ corresponds to mode 4], and two runs intended to test the code. Two of the high-resolution multimode runs include only a short wavelength spectral component with modes 20-80, given by a narrow gaussian in two cases and a $1 / \mathrm{k}^{2}$ spectrum in the other (see Fig. 2). Each interface was constructed by determining all modes in the annulus satisfying $20 \leq \operatorname{sqrt}\left(\mathrm{k}_{\mathrm{x}}^{2}+\mathrm{k}_{\mathrm{y}}{ }^{2}\right) \leq 80$ (for integer $\mathrm{k}_{\mathrm{x}}$ 


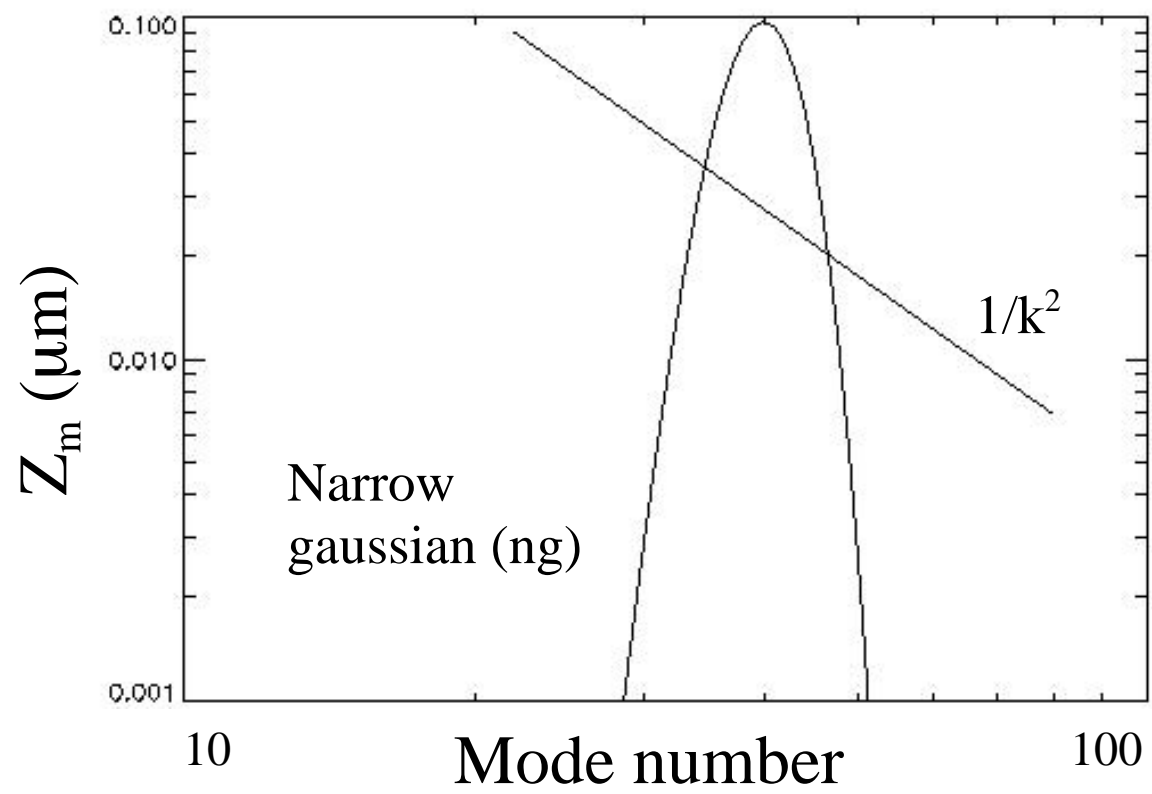

Figure 2: Initial spectral shapes used for the short-wavelength component in the 3D calculations.

and $\mathrm{k}_{\mathrm{y}}$ ) and assigning to each a random phase and randomized amplitude taken from the prescribed spectrum. Contour plots of the initial conditions are shown in Figs. 3(a)-3(b). The other two multimode calculations include a simple long wavelength component in addition to the narrow gaussian short wavelength component [see Figs. 3(c)-3(d)]. The long wavelength component is either a single 2D mode 4 or the 3D mode 4 "egg crate".

In estimating the Reynolds number in the calculations, we take $\operatorname{Re}_{\mathrm{h}}(\mathrm{t}) \equiv$ $[2 \mathrm{~h}(\mathrm{t}) / \Delta \mathrm{x}]^{4 / 3}$ where $2 \mathrm{~h}(\mathrm{t})$ is the mix width and $\Delta \mathrm{x}$ is the cell size. This give a timedependent Reynolds number that climbs as high as $\sim 5500$ in the first $18 \mathrm{~ns}$ of a $40 \mathrm{~ns}$ experiment. This is the same order of magnitude as the time-independent Reynolds number based on the width of the computational domain $\mathrm{Re}_{\mathrm{L}}=1626$. If the perturbation amplitude growth is similar in $2 \mathrm{D}$ and $3 \mathrm{D}$, then we expect to find $\mathrm{Re}_{\mathrm{h}} \sim$ 


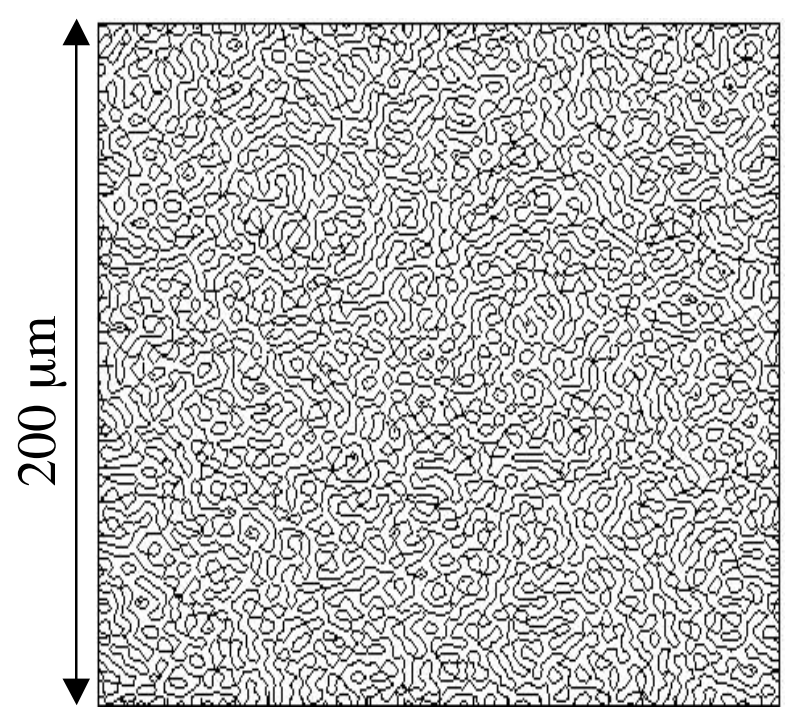

(a)

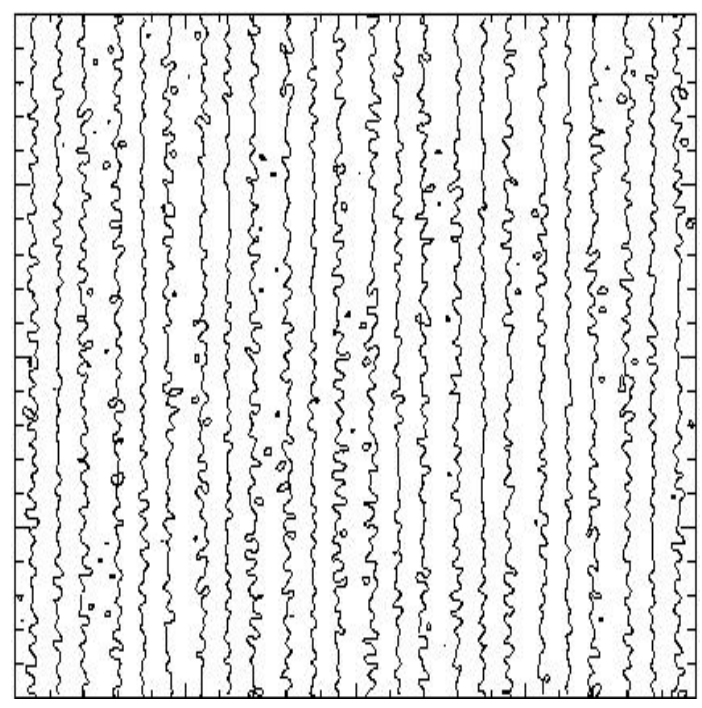

(c)

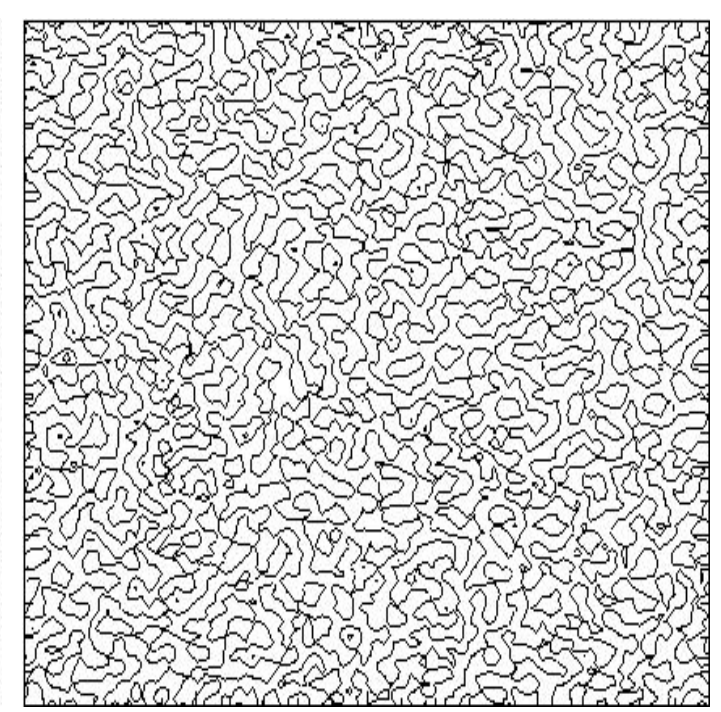

(b)

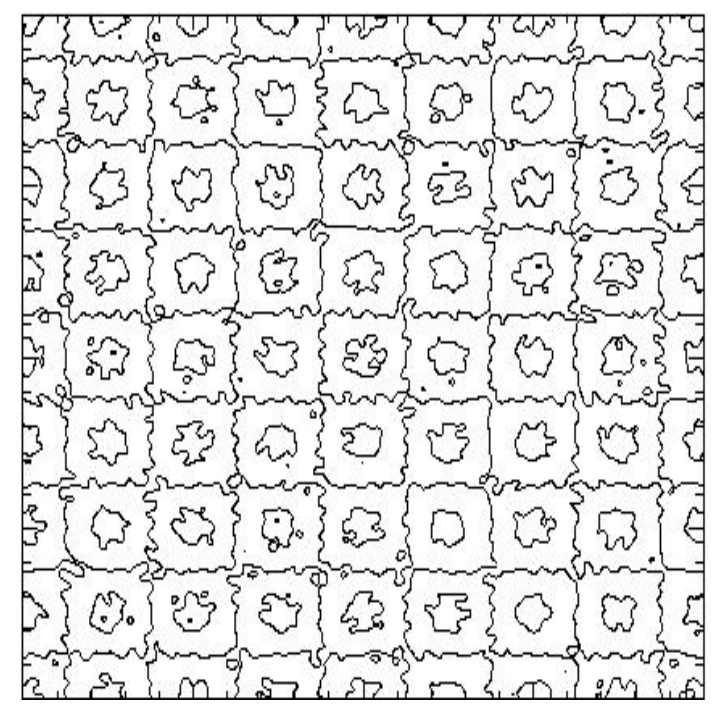

(d)

Figure 3: Initial interface contour plots: (a) narrow gaussian (ng), (b) $1 / \mathrm{k}^{2}$, (c) narrow gaussian shorts plus 2D mode 4, and (d) narrow gaussian shorts plus mode 4 "egg crate".

11000 at $40 \mathrm{~ns}$. The estimated Re number in the actual experiments is of order $10^{5}$, or two orders of magnitude greater than in the simulations. ${ }^{13}$ Typical of numerical simulations of turbulent systems, the dissipative scales are much smaller than the grid scale. We might expect, however, to qualitatively capture the effect of the turbulence 
on the large scales if we adequately resolve the lower end of a self-similar inertial range. According to Dimotakis, this scale can be estimated by the Liepmann-Taylor scale, which he defines as $\lambda_{\mathrm{LT}}=5 \mathrm{LRe}_{\mathrm{h}}^{-3 / 4}$ where $\mathrm{L}$ is the driving scale. ${ }^{10}$ With $\mathrm{L}=2 \mathrm{~h}$, we find for the experiments $\lambda_{\mathrm{LT}} \approx 3-10 \mu \mathrm{m}$. In the simulations, there are then about 413 cells per Liepmann-Taylor scale, which corresponds to mode 67-20. Thus we estimate that we are beginning to resolve the upper end of the inertial range with 256 ppb. We would feel much more comfortable with another factor of 2-4 in resolution, but such calculations are for now prohibitively time-consuming.

\section{Simulation results}

\section{A. Test calculations}

Two calculations were run to test the 3D version of Raptor adapted for use in this study. In the first, an interface was generated from a uniform 2D spectrum including modes 4 through 80 . The purpose of this test was to see when numerical noise would introduce observable $3 \mathrm{D}$ effects into the simulation. The problem was run out to $8.7 \mathrm{~ns}$, at which point the width of the mix region was about 24 times the initial characteristic wavelength (see Fig. 4). Despite this high degree of nonlinearity and a complicated interface structure, there were absolutely no indications of 3D flow even at the end of the calculation. Thus a simulation that begins $2 \mathrm{D}$ will remain $2 \mathrm{D}$ well into the nonlinear regime.

Since the first test involved grid-aligned perturbations, we ran in second test in which a single mode 4 perturbation was set up with its wavevector rotated by 45 


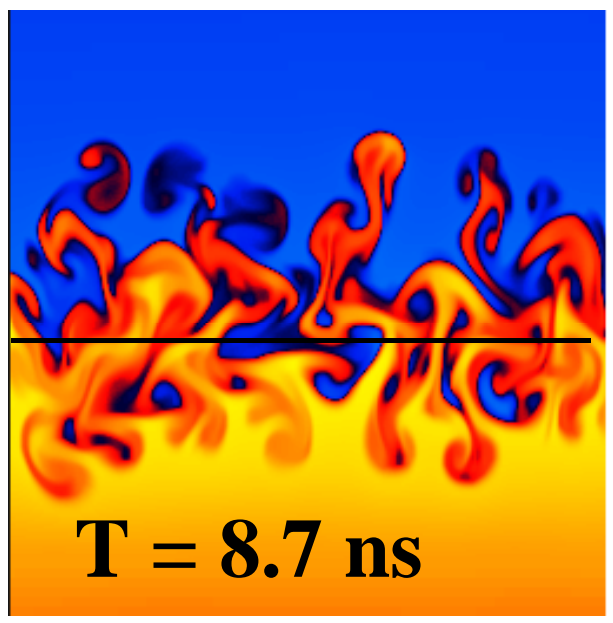

(a)

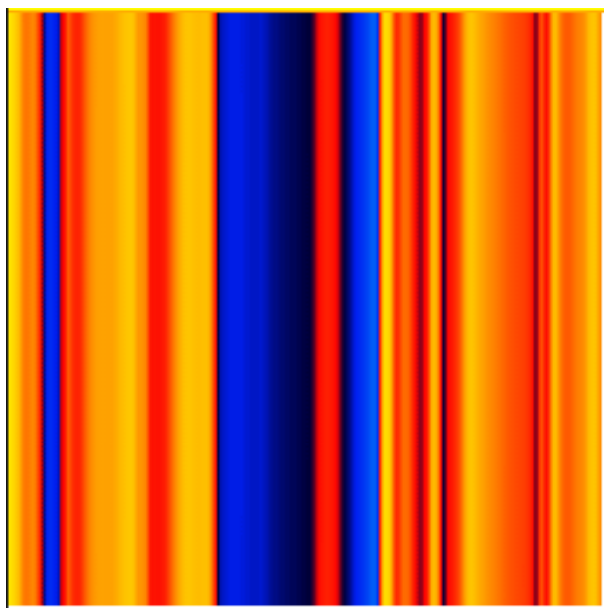

(b)

Figure 4: Log density slices through a 3D simulation with 2D initial conditions from a uniform (flat) spectrum including modes 4 through 80 . The dark line in (a) denotes the location of (b) the horizontal slice. The initial rms amplitude in this calculation was $0.32 \mu \mathrm{m}$.

degrees about the vertical axis. The calculation was run out to $14 \mathrm{~ns}$, at which point the nonlinearity was $a / \lambda=1.27$. The flow at the boundaries was corrupted due to the periodic boundary conditions, but the perturbation away from the walls remained single mode despite the fact that is was non grid-aligned (see Fig. 5). 


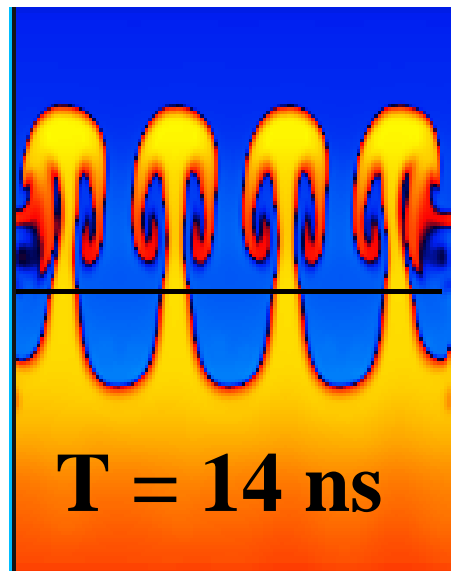

(a)

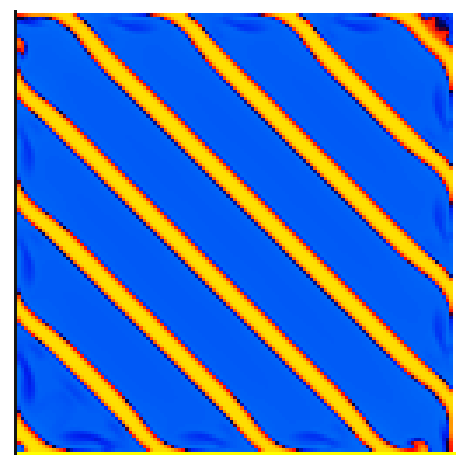

(b)

Figure 5: Log density slices through a 3D simulation with initial conditions given by a single mode (mode 4 ) oriented at $45^{\circ}$. The dark line in (a) denotes the location of (b) the horizontal slice. The resolution in this calculation was 128 cells in the transverse direction and the initial perturbation amplitude was $1.25 \mu \mathrm{m}$.

\section{B. Effect of dimensionality on single mode growth}

In Fig. 6, we compare the growth of the mode 4 "egg-crate" with a single 2D mode 4 . The $3 \mathrm{D}$ spikes and bubbles have the same transverse scale as the $2 \mathrm{D}$ spikes and bubbles, so, during the pre-transition phase, the difference in their growth rates should be set by the ratio of their drag coefficients ${ }^{25,30,31}$ $u_{3 D} / u_{2 D}=\sqrt{C_{2 D} / C_{3 D}} \approx \sqrt{6 \pi /(2 \pi \cdot 1.22)} \approx \sqrt{2.46} \approx 1.57$. In fact, the 3D growth rate 


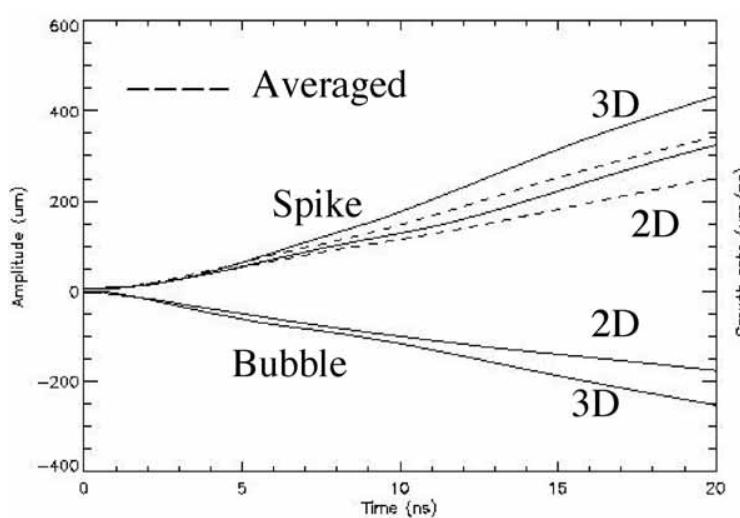

(a)

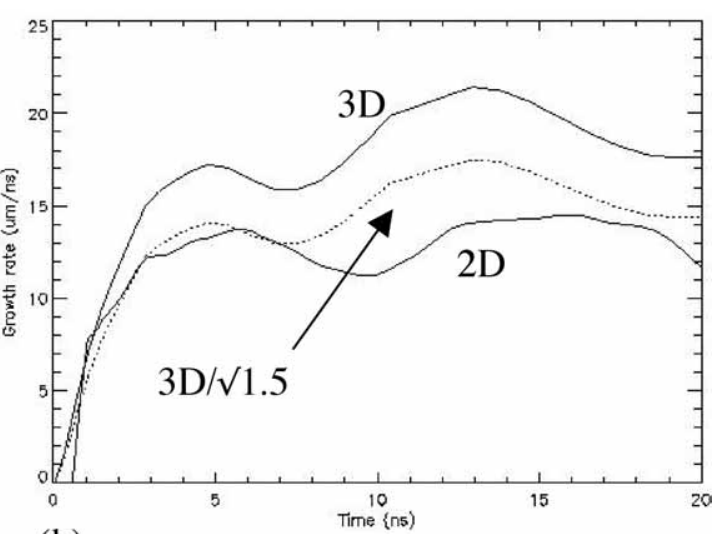

(b)
3D

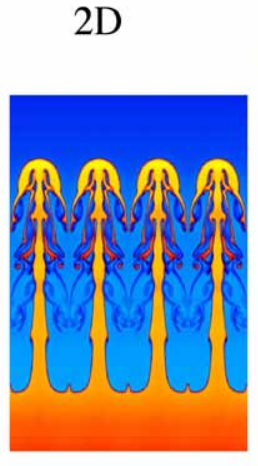

$7.9 \mathrm{~ns}$

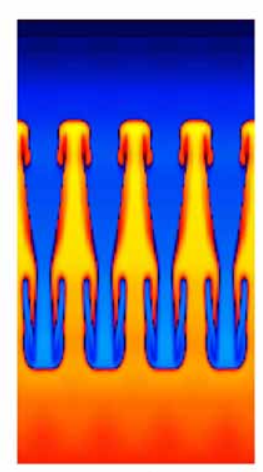

$7.5 \mathrm{~ns}$

(c)

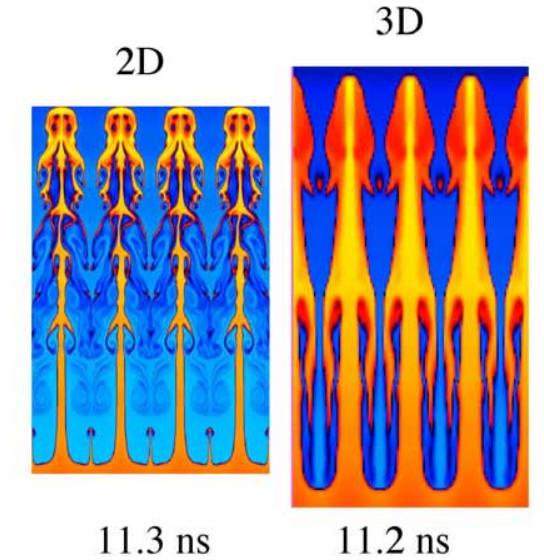

$11.3 \mathrm{~ns}$

$11.2 \mathrm{~ns}$

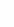




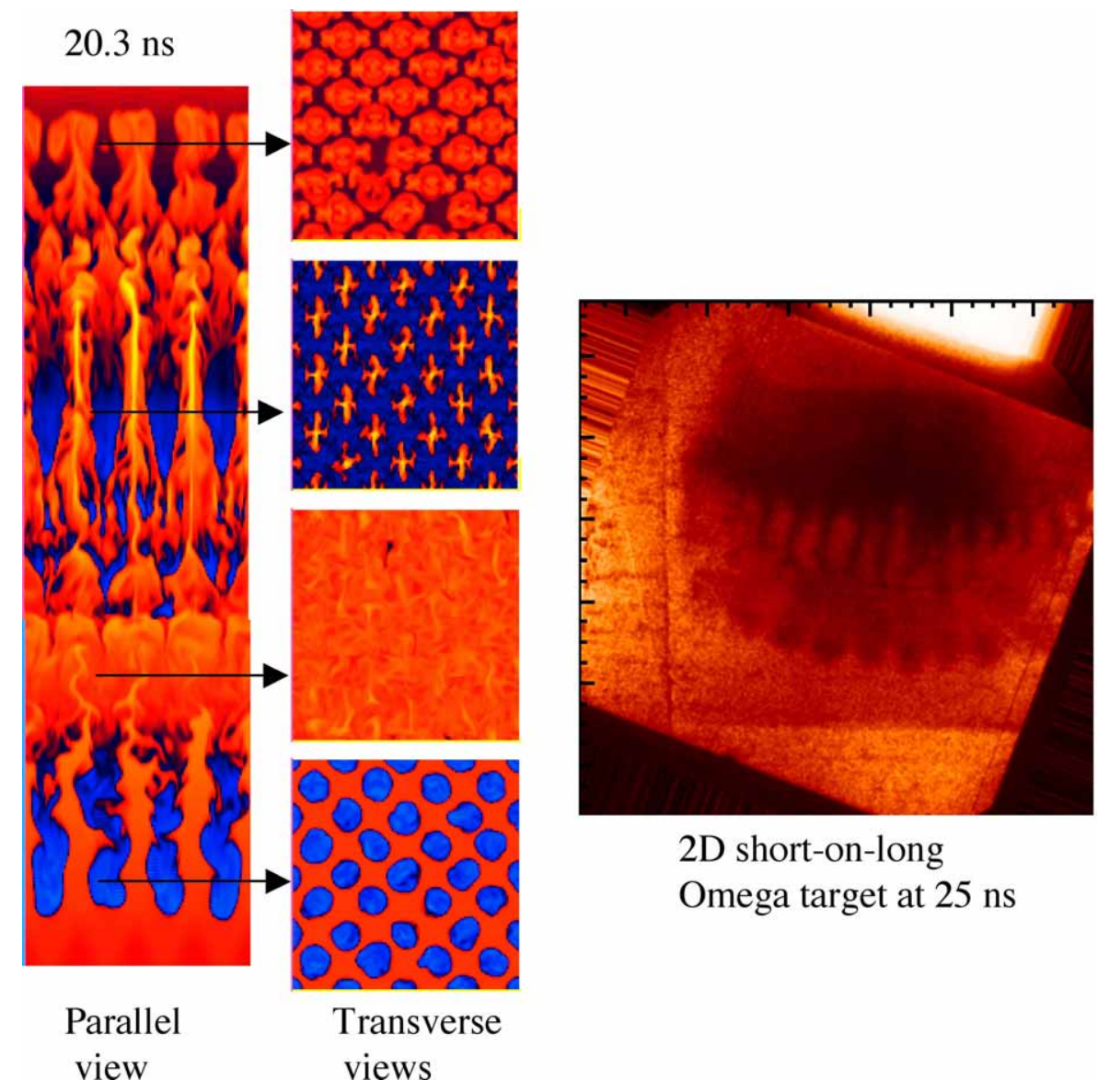

Figure 7: Late-time log density image from mode 4 "egg-carton" interface. Despite nonlinearity $\mathrm{a} / \lambda=7$, regular single-scale structure persists along much of the length of the spikes. However, a transition to turbulence appears to have occurred in localized regions where shear layers from individual spikes have merged. An x-ray radiograph at $25 \mathrm{~ns}$ from a 2D short-on-long Omega target shows indications of similar localized transitions. The perturbation in the experiment is less nonlinear both because it is $2 \mathrm{D}$ and the drive is five times less energetic.

shows indications of similar localized transitions. The perturbation in the experiment is less nonlinear both because it is $2 \mathrm{D}$ and the drive is five times less energetic. Based on the time required for establishment of an inertial range via the viscous growth of the Liepmann-Taylor scale, Robey et al. predict that the mixing transition is possible in the experiments after $17 \mathrm{~ns} .{ }^{13}$ This scenario seems appropriate in this spectrally simple system where there is no weak transition early on. 


\section{Evolution of short-wavelength component}

Perturbation amplitude and velocity histories from the two high-resolution 3D shorts-only cases are shown in Fig. 8. Results from 2D calculations with the same initial spectral shapes are shown for comparison. In general, the variation between the $3 \mathrm{D}$ calculations is slightly less than in $2 \mathrm{D}$. This could be due at least in part to better statistics in the identification of the spike and bubble positions.

At early times, 3D bubbles grow faster than 2D bubbles of the same size. This is expected based on the reduced drag of spherical relative to cylindrical bubbles. At about $5 \mathrm{~ns}$, however, the 3D bubble velocities begin to drop off rapidly, falling to about half the 2D bubble velocity by $10 \mathrm{~ns}$.

Within a few ns after shock transmission, a transition begins from a state with little small-scale mixing to a highly-mixed state [see Fig. 9(a)]. The degree of "mixedness" is determined by using Youngs's "molecular mix", defined by ${ }^{33}$

$$
\Theta \equiv \frac{\int\langle f(1-f)\rangle d z}{\int\langle f\rangle d z \int\langle 1-f\rangle d z}
$$

where $\mathrm{f}$ and 1-f are the volume fractions of the two fluids and the angular brackets denote averaging over the transverse direction. In the $2 \mathrm{D}$ calculations, this " $2 \mathrm{D}$ mixing transition" is complete when the mixing parameter begins to saturate at $4 \mathrm{~ns}$ at a level of about 0.7 . As we noted previously, this is noticeably higher than the value of about 0.54 obtained by Youngs and Cook et al. from their 2D simulations of classical, incompressible RT. ${ }^{24,34}$ In $3 \mathrm{D}$, the mixing parameter continues to increase until about $10 \mathrm{~ns}$, when it begins to saturate at a higher value of about 0.90. Again, this is higher than the value of about 0.83 reported by Youngs and Cook et al. ${ }^{24,34}$ 

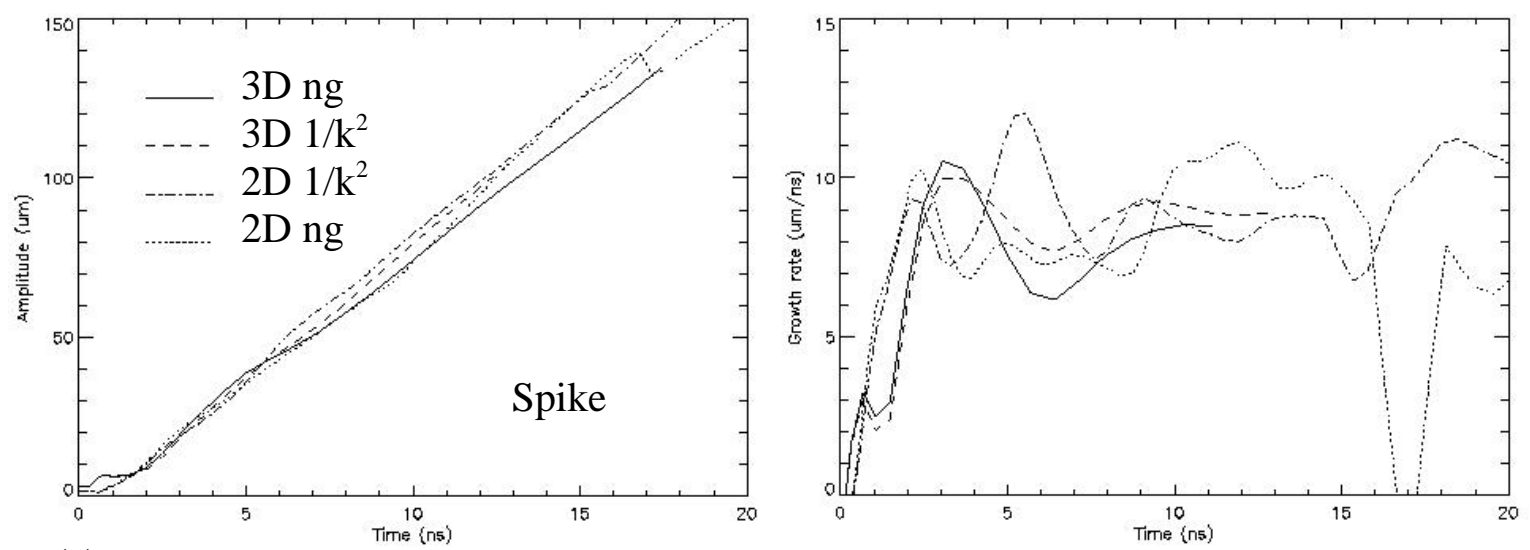

(a)
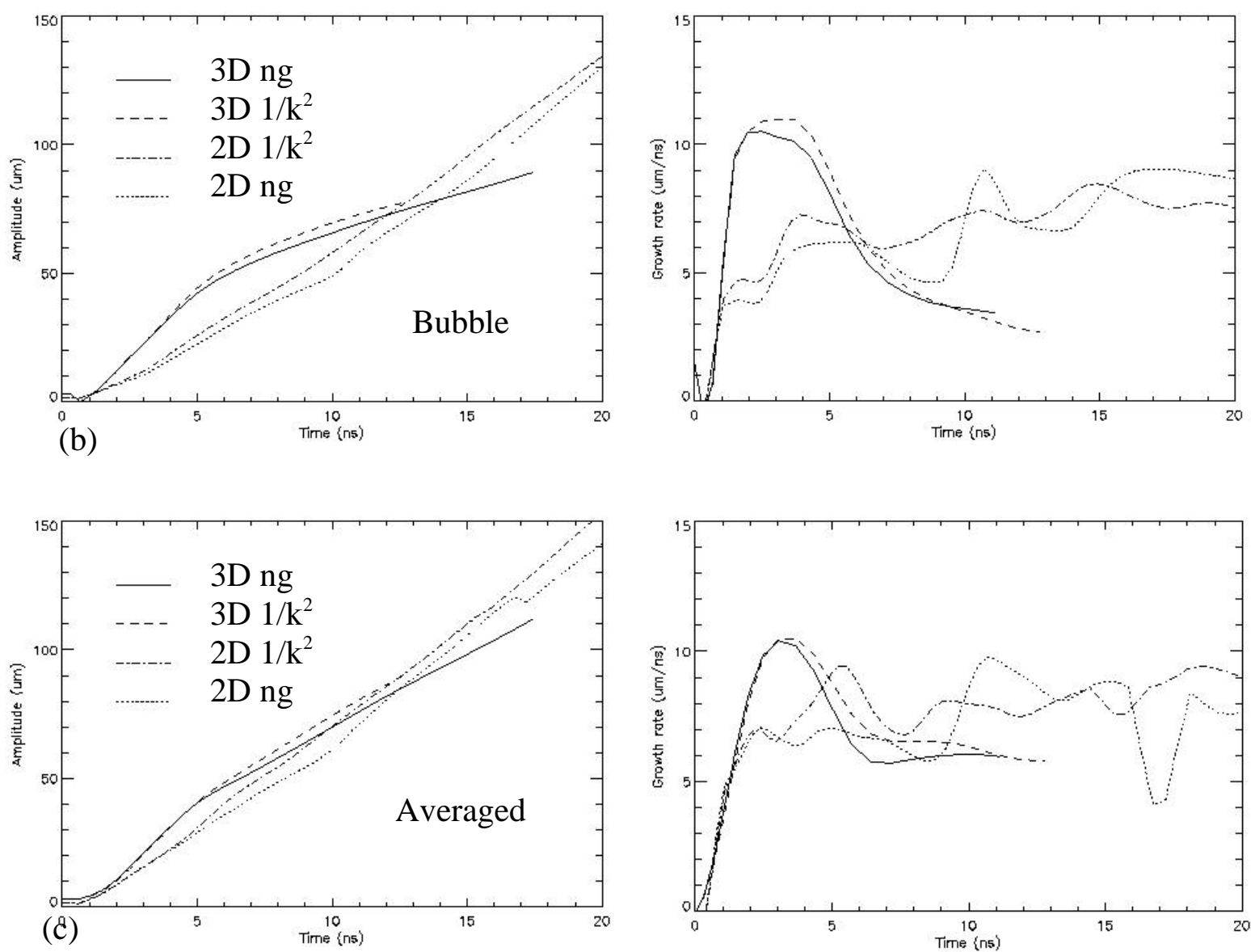

Figure 8: Perturbation amplitude and velocity histories from narrow gaussian and $1 / \mathrm{k}^{2}$ shorts-only cases. Results from 2D calculations (resolved to $512 \mathrm{ppb}$ ) with the same spectral shapes are included for comparison. 
Three-dimensional Raptor simulations of classical RT do not show this discrepancy, ${ }^{35}$ suggesting that it results from RM, thermal conduction, decompression, or a combination of these effects. We have verified that conduction is not responsible, and do not believe that RM is important after the first few ns. This leaves decompression, which tends to increase the number of mixed zones at material interfaces. We have not yet determined whether the resulting increase in asymptotic mixedness is a physical or numerical effect.

By about $10 \mathrm{~ns}$, the simulation appears to have undergone a turbulent mixing transition (see Fig. 9). Indeed, at $90 \%$ mixed, it is difficult to imagine what additional mixing transition could possibly take place.

Two-dimensional density and energy spectra are integrated over annuli in $\mathrm{k}_{\mathrm{x}}{ }^{-}$ $\mathrm{k}_{\mathrm{y}}$ space to give a $1 \mathrm{D}$ representation that depends only on the magnitude of the transverse wavevector. Results from the 3D narrow-gaussian case are shown in Fig. 10. The density fluctuation is defined by $\delta \rho(z)=\rho(z)-\langle\rho(z)>$, where $<\rho(z)>$ is the $z-$ dependent transverse density average. Each spectrum shown in Fig. 10 is the average of several (typically four) 1D spectra evenly spaced throughout the interior of the mix region. The inverse cascade to longer wavelengths is apparent in the density spectra. It proceeds rapidly at early times but slows dramatically by $20 \mathrm{~ns}$, indicating the approach of the asymptotic freeze-out stage. This slowing is more apparent in 3D than in our earlier 2D calculations, where vortex pairing and merger contributes to the inverse cascade.

The fluctuating energy components are defined in the same way as the density fluctuation: $\delta \operatorname{KE}_{\mathrm{x}, \mathrm{y}, \mathrm{z}}(\mathrm{z})=\mathrm{KE}_{\mathrm{x}, \mathrm{y}, \mathrm{z}}(\mathrm{z})-<\mathrm{KE}_{\mathrm{x}, \mathrm{y}, \mathrm{z}}(\mathrm{z})>$, where "<>" again denotes 


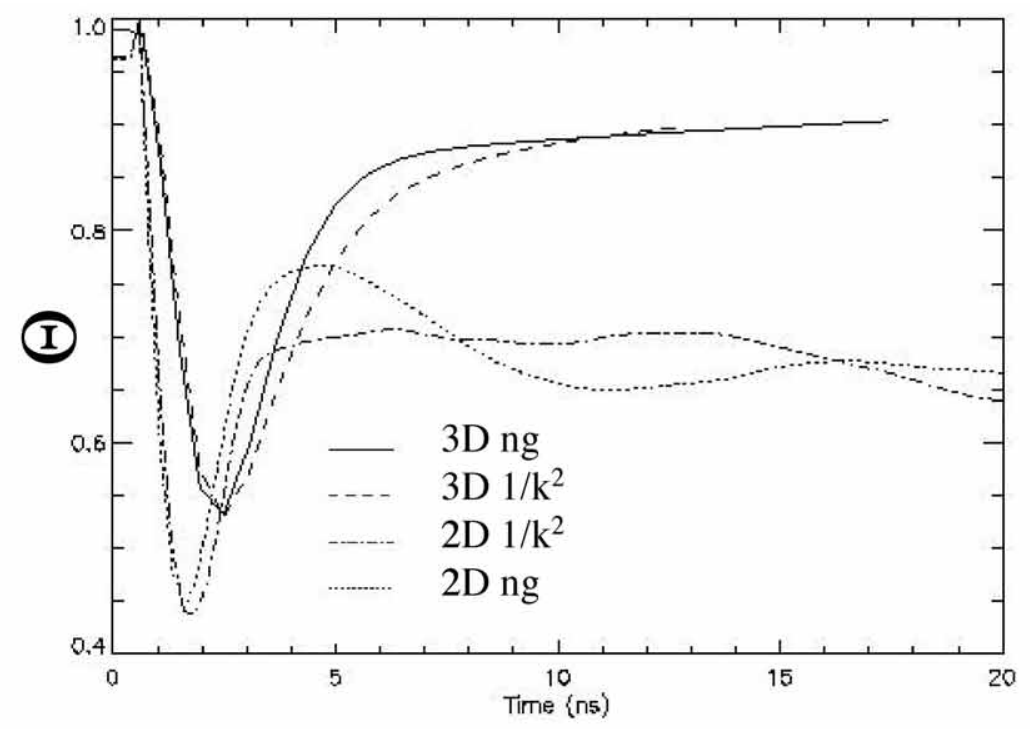

(a)

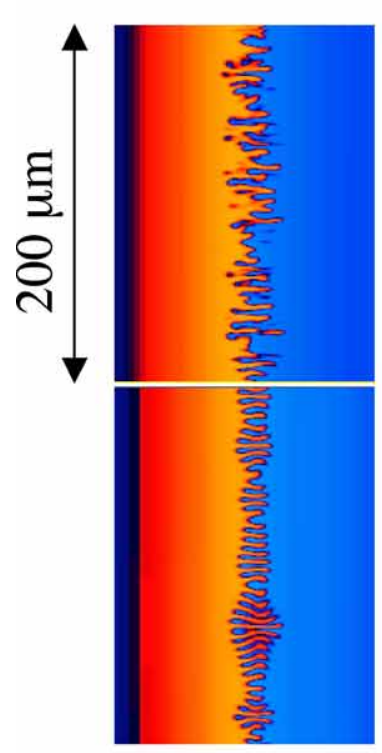

$2.5 \mathrm{~ns}$

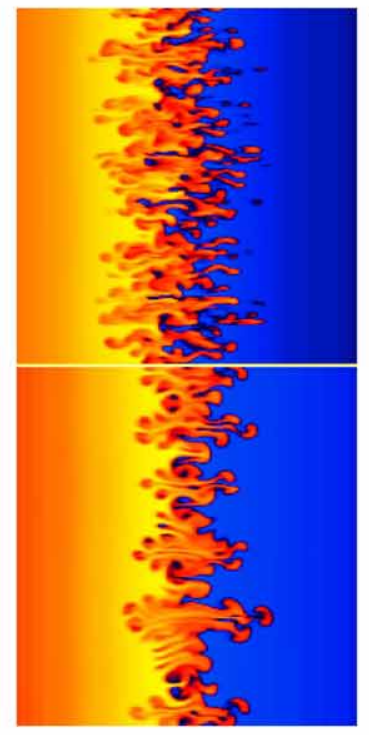

$5.0 \mathrm{~ns}$

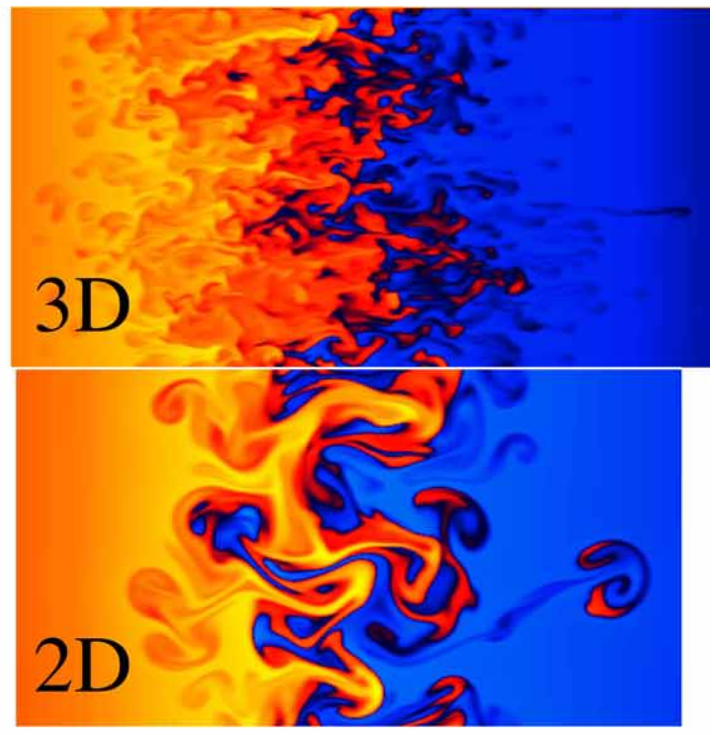

$17.5 \mathrm{~ns}$

(b)

Figure 9: (a) Mixing parameter and (b) log density plots from 2D and 3D ng shortsonly calculations. 


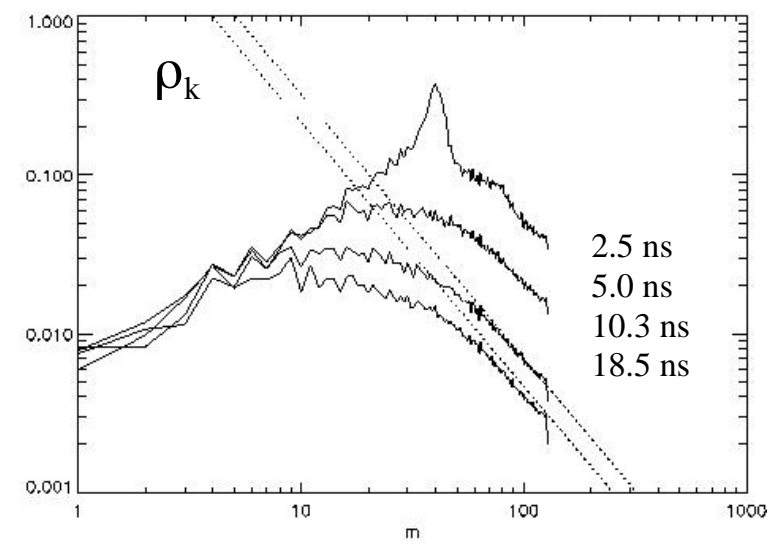

(a)

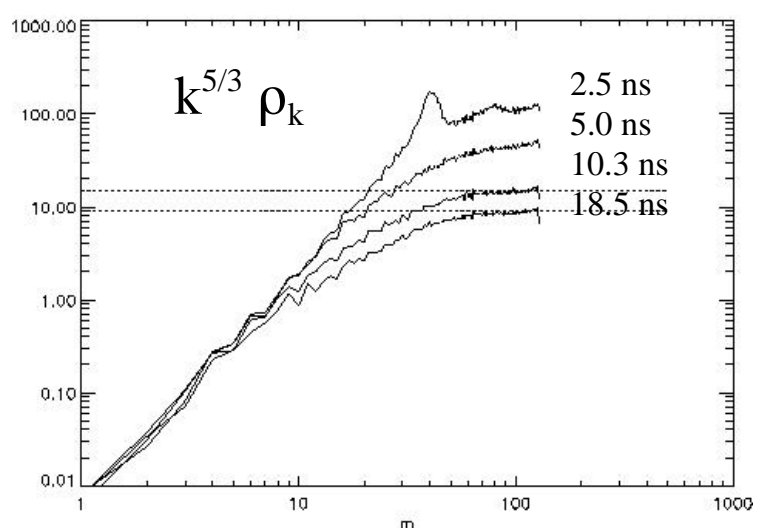

(b)

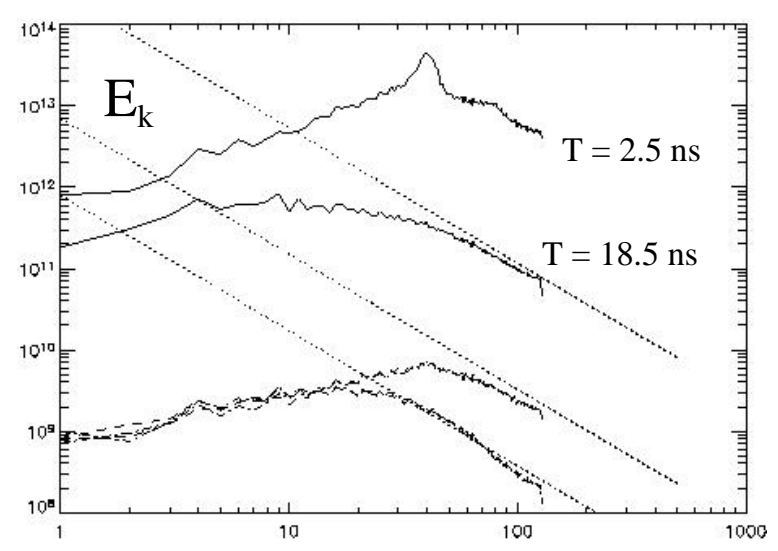

(c)

m

Parallel

Transverse $\mathrm{x}$

Transverse y

Figure 10: Time evolution of (a) density spectra $\rho_{k}$, (b) $k^{5 / 3} \rho_{k}$, and (c) turbulent directed energy spectra.The appearance of an inertial range in the spectra corresponds to the increase in mixedness apparent in Fig. 9(a). 
transverse average and $\mathrm{KE}_{\mathrm{x}, \mathrm{y}, \mathrm{z}}=\rho \mathrm{v}_{\mathrm{x}, \mathrm{y}, \mathrm{z}}{ }^{2}$. An inertial range with Kolmogorov $\mathrm{k}^{-5 / 3}$ scaling $^{36}$ is visible by about $10 \mathrm{~ns}$ in the density and parallel energy spectra from about mode 70 out to about 120 . The appearance of an inertial range in the transverse energy spectra is somewhat delayed relative to the parallel component. This effect has been previously noted in 3D classical RT simulations and attributed to the secondary nature of the transverse flow. ${ }^{24}$ That an inertial range appears at all is at first glance surprising considering the relatively limited range of scales present in these simulations and the lack of a sub-grid-scale model. The grid-resolution Reynolds number, defined by $\operatorname{Re}_{\Delta x} \equiv(h / \Delta x)^{4 / 3} \sim 10^{3}$, is an order of magnitude smaller than the integral-scale Reynolds number observed by Dimotakis to mark the mixing transition in many flows. ${ }^{10} \mathrm{We}$ believe that an inertial range is attainable in our case because the dissipation range, which is almost imperceptible in the post-transition density and transverse energy spectra, occupies only a few cells. Because the Kolmogorov scaling is not corrupted at the high-k end by dissipation, the effective integral-scale Reynolds number is much higher than the grid-resolution Reynolds number. Following the scaling used by Dimotakis, we find that the ratio of the lower end to the upper end of the inertial range is approximately given by $0.1 \mathrm{Re}^{1 / 4}$. Based on the inertial range observed in the calculated spectra, this gives $\operatorname{Re} \approx 5 \times 10^{4}$. As predicted by Dimotakis, the appearance of the inertial range corresponds with a turbulent mixing transition.

It is important to note that the $3 \mathrm{D}$ bubble velocity falls off precisely while the $3 \mathrm{D}$ mixedness is increasing above the value seen in the $2 \mathrm{D}$ calculations. This suggests 
that the increased mixing in the bubble region has resulted in a lower effective Atwood number and, consequently, a lower growth rate.

In this spectrally complex system, the time to transition does not seem to be limited by the viscous growth of a shear layer as was suggested in the 3D singlemode calculation. Instead, transition is triggered by strong interactions between neighboring spikes. When the dominant spikes reach their nonlinear breakdown thresholds, a significant fraction of their energy is diverted into the transverse direction, resulting in forcing of mix-layer-scale vortices. Since this happens earlier when the characteristic mode number in the spectrum is higher, the transition here takes place earlier than it does in the single-mode system despite the fact that the integral scale (the mix width) is much smaller.

\section{Effect of shorts on long}

Two high-resolution, 3D, short on long simulations were run. In each case, the short wavelength component was the same 3D narrow gaussian shown in Figs. 2 and 3(a) and used in Fig. 9. The long-wavelength component was a 2D mode 4 in one case and a mode 4 "egg carton" (which we will call 3D mode 4) in the other. Amplitude and velocity histories from these calculations are shown in Fig. 11 along with results from $2 \mathrm{D}$ mode $4,3 \mathrm{D}$ mode 4 , and 2D narrow gaussian shorts on a $2 \mathrm{D}$ mode 4 . In each case, the amplitude of mode 4 was $2.5 \mu \mathrm{m}(\mathrm{a} / \lambda=0.05)$, and the rms amplitude of the short wavelength component was ten times smaller.

The large reduction in bubble growth seen with the short-wavelength spectrum is not so apparent when mode 4 is present [see Fig 11(b)]. We believe that 
this is because it takes much longer for mode 4 to grow up to its nonlinear interaction and breakdown thresholds. Consequently, the mixing transition does not occur until
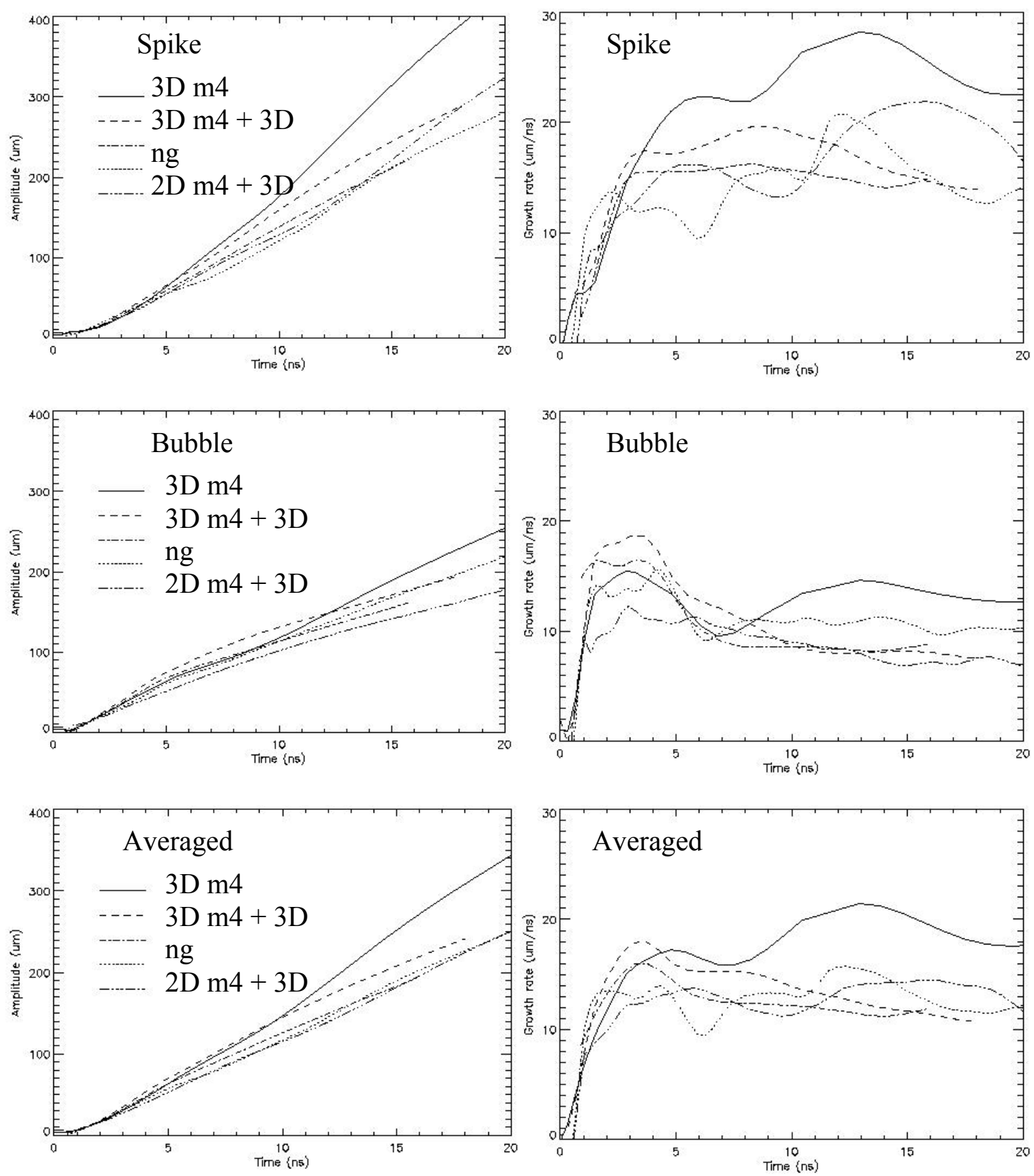
Figure 11: Perturbation amplitude and velocity histories from calculations with mode $4(\mathrm{~m} 4)$ in the initial conditions. Results from 2D calculations with the same spectral shapes are included for comparison.

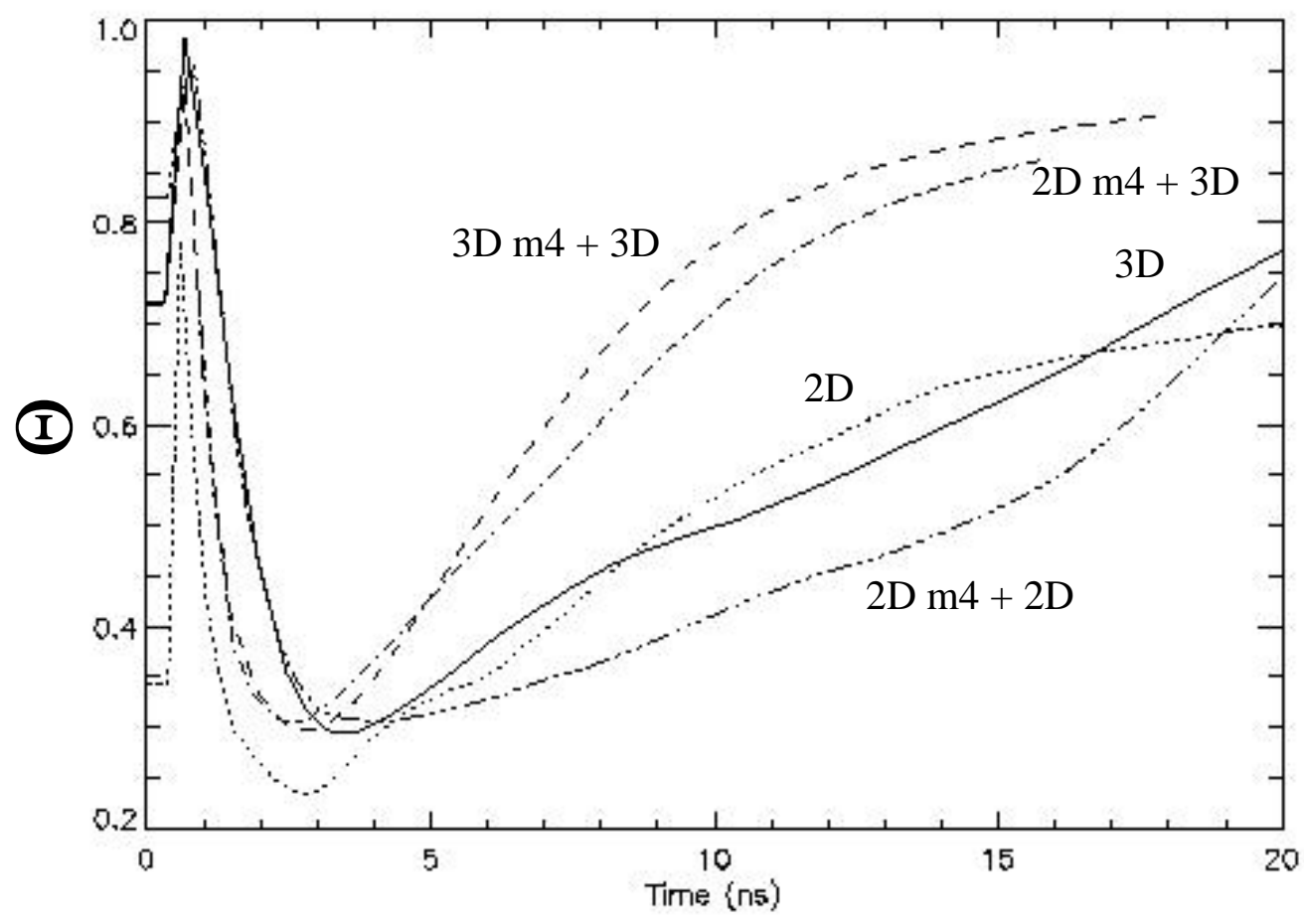

Figure 12: Mixing in simulations with mode $4(\mathrm{~m} 4)$ in the initial spectra. The 2D calculations show evidence of weak transition at around $20 \mathrm{~ns}$. The 3D single-mode "egg-carton" perturbation also shows a transition at around $20 \mathrm{~ns}$, which is the same time that log density plots appear to show localized mixing transitions. When 3D noise is present, the transition happens much earlier (between 10 and $15 \mathrm{~ns}$ ).

between 10 and 15 ns (see Fig. 12). This is consistent with the conclusion we made based on our 2D calculations that the presence of a long-wavelength mode with significant initial amplitude can delay the transition to a turbulent-like state.

Log density slices through the evolving mix layer from the 3D m4 plus 3D narrow gaussian shorts calculation are shown in Fig. 13. The dominant mode 4 spikes begin to interact with one another when $\mathrm{a} / \lambda \approx 2$. A very clear transition to a wellmixed state has taken place by $18 \mathrm{~ns}$, at which point $\mathrm{a} / \lambda \approx 5$. These same nonlinear 
thresholds for spike interaction and breakdown/transition were found in our 2D calculations. ${ }^{6}$ Thus it appears that, for complex initial spectra, transition in blast-

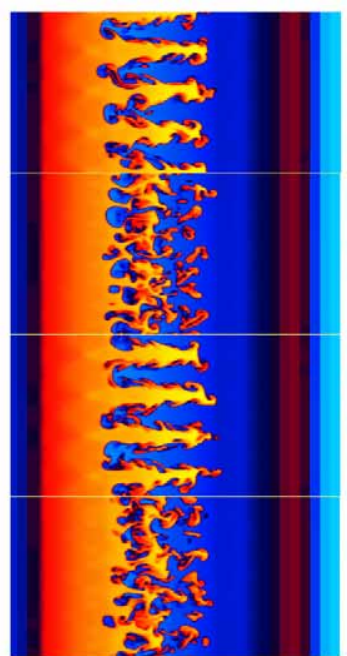

$4.9 \mathrm{~ns}$ $\mathrm{a} / \lambda \sim 1.5$

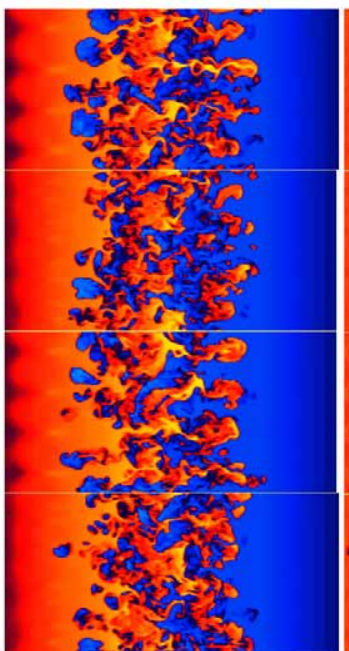

$8.2 \mathrm{~ns}$ $\mathrm{a} / \lambda \sim 2.2$

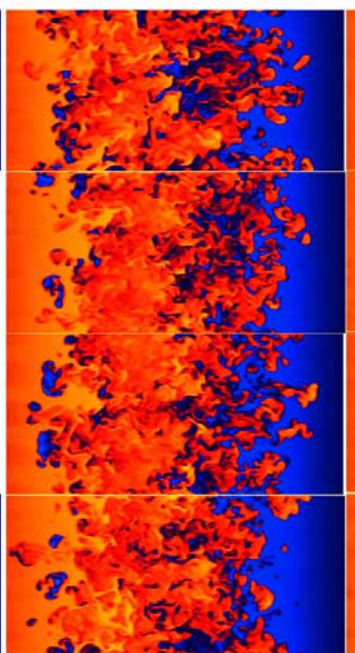

$12.0 \mathrm{~ns}$ $\mathrm{a} / \lambda \sim 3.5$

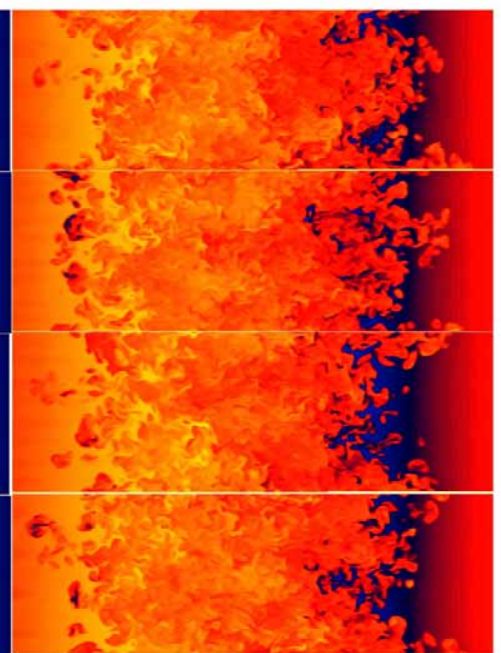

$18.0 \mathrm{~ns}$ $\mathrm{a} / \lambda \sim 5.0$

Figure 13: Log density plots of the evolving mix layer from the 3D m4 plus 3D narrow gaussian shorts calculation. Spike interaction (between the mode 4 spikes) begins when $\mathrm{a} / \lambda \approx 2$. A very clear transition to a well-mixed state has taken place by $18 \mathrm{~ns}$, at which point $\mathrm{a} / \lambda \approx 5$.

wave-driven systems proceeds similarly in $2 \mathrm{D}$ and $3 \mathrm{D}$, indicating that threedimensional secondary instabilities do not play a dominant role in initiating the transition. Again, the mixing transition corresponds to the loss of transverse spectral information and the appearance of an inertial range with $\mathrm{k}^{-5 / 3}$ scaling (see Fig. 14).

The 2D mode 4 plus 3D narrow gaussian shorts evolves similarly, except that the interior of the mix region remains anisotropic in the transverse plane until the mixing transition has taken place (see Figs. 15-16). The post-transition mix width remains somewhat smaller than with the egg-carton perturbation, but the two flows are otherwise very difficult to distinguish. 

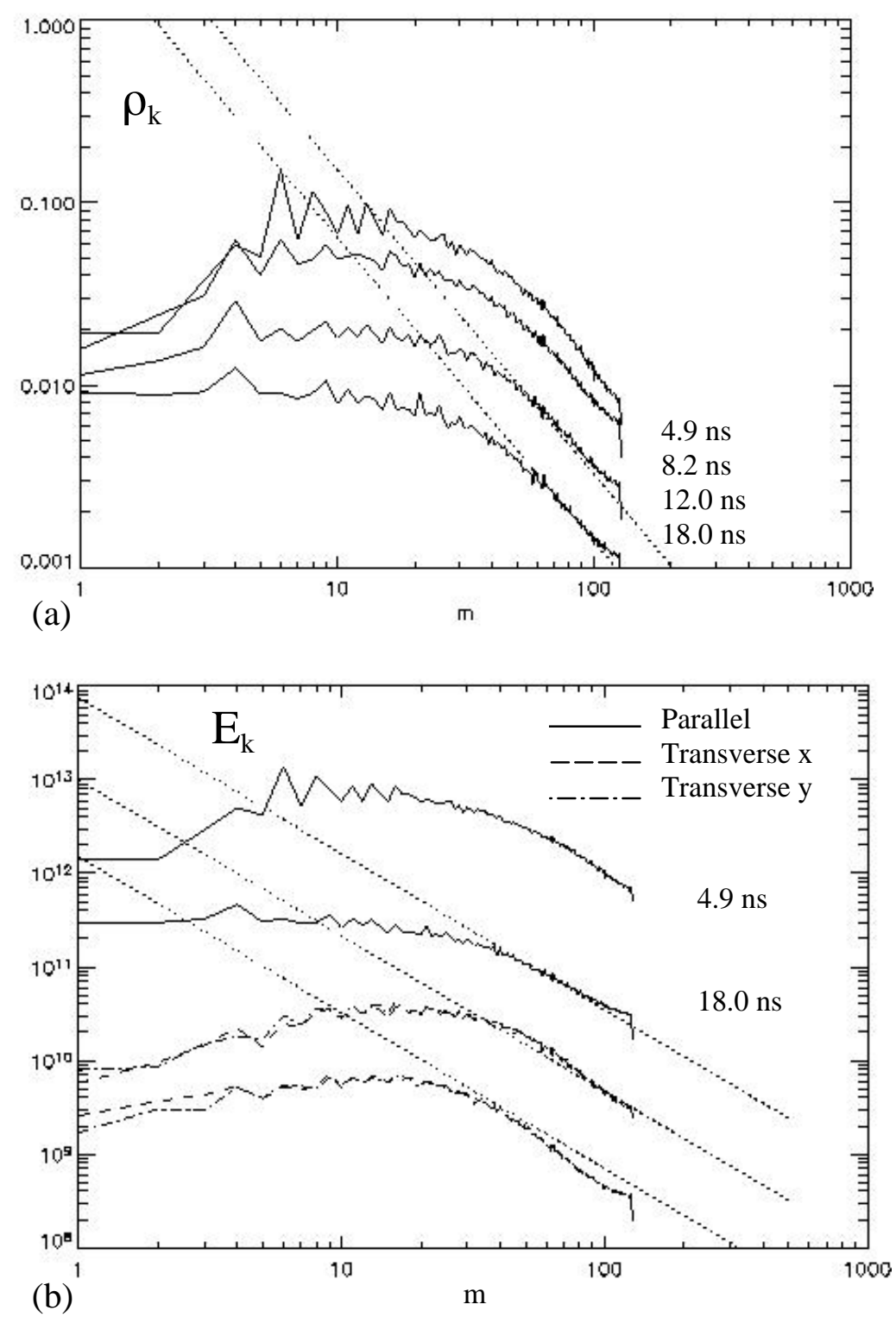

Figure 14: (a) Density and (b) energy spectra from the 3D m4 plus 3D narrow gaussian shorts calculation. An inertial range is apparent by the time the mixing transition has taken place. 

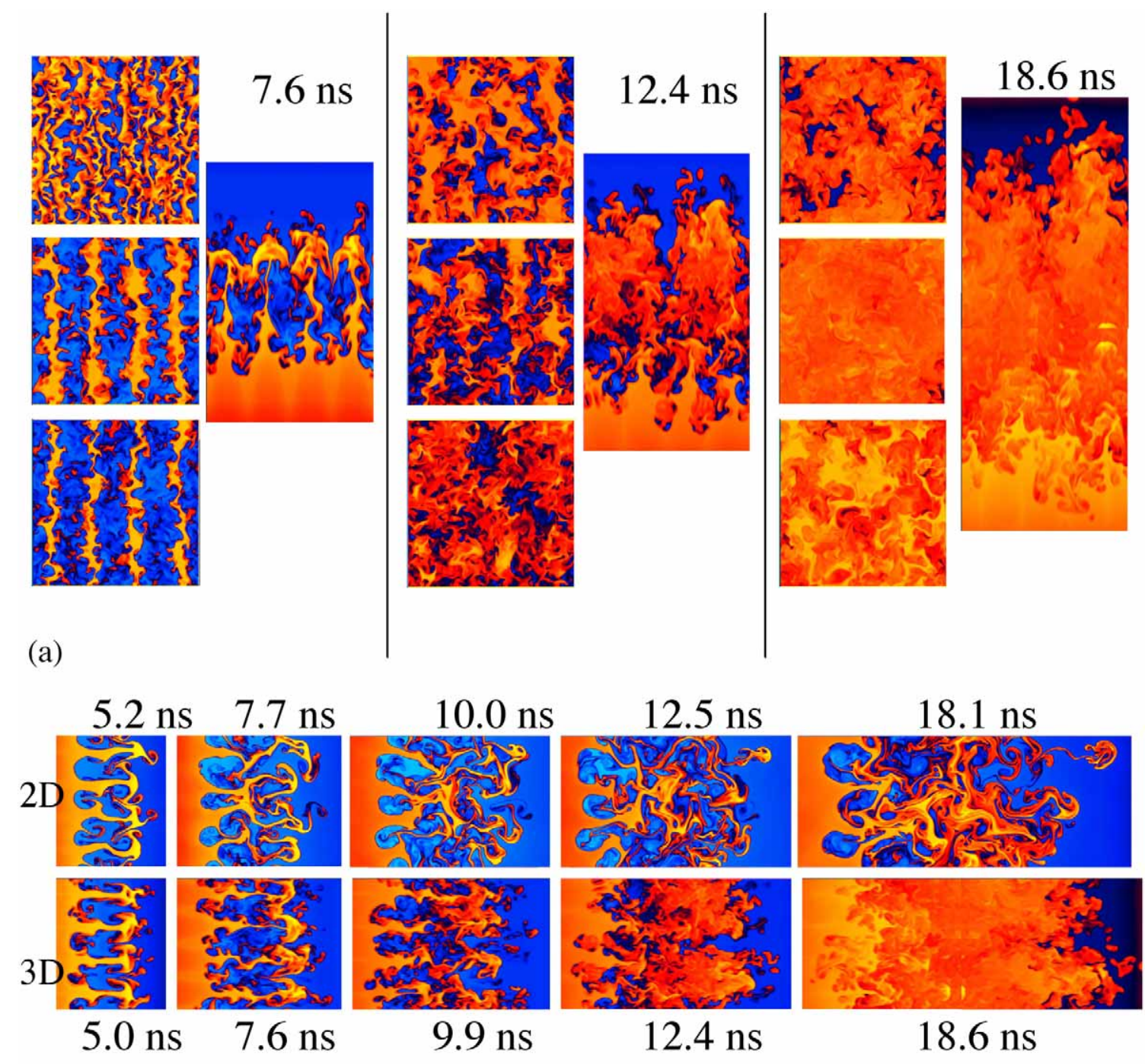

(b)

Figure 15:(a) Log density slices of the evolving mix layer from the 2D m4 plus 3D narrow gaussian shorts calculation. The interior of the mix region remains anisotropic in the transverse plane until the mixing transition has taken place. (b) Comparison with the analogous 2D calculation (2D m4 plus 2D narrow gaussian shorts) illustrates 
the importance of 3D effects.

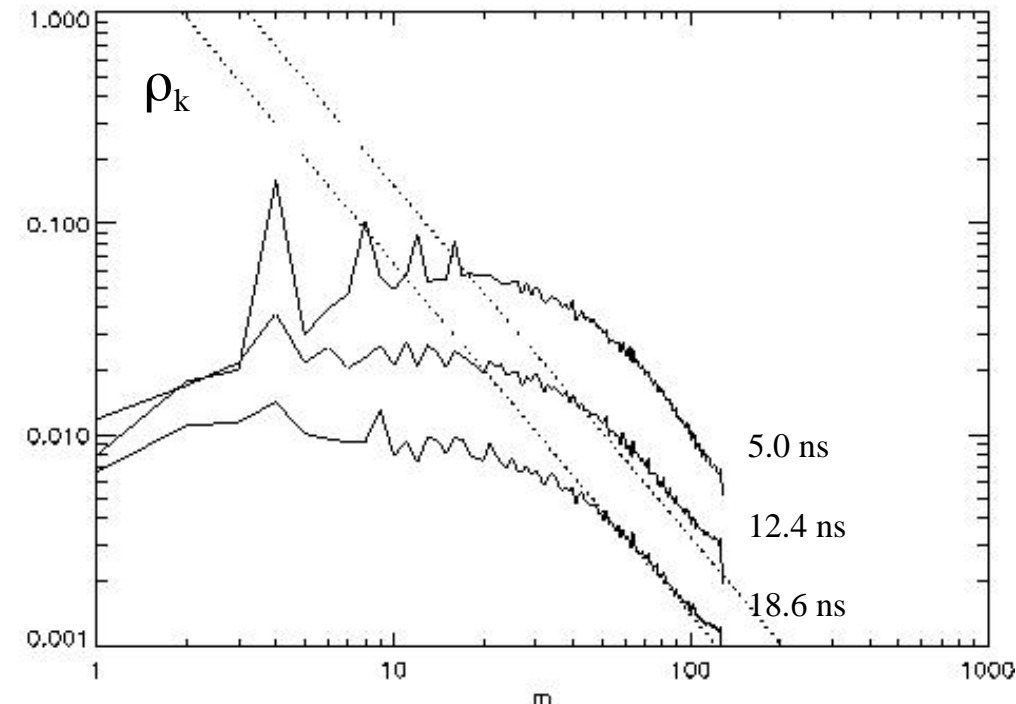

(a)

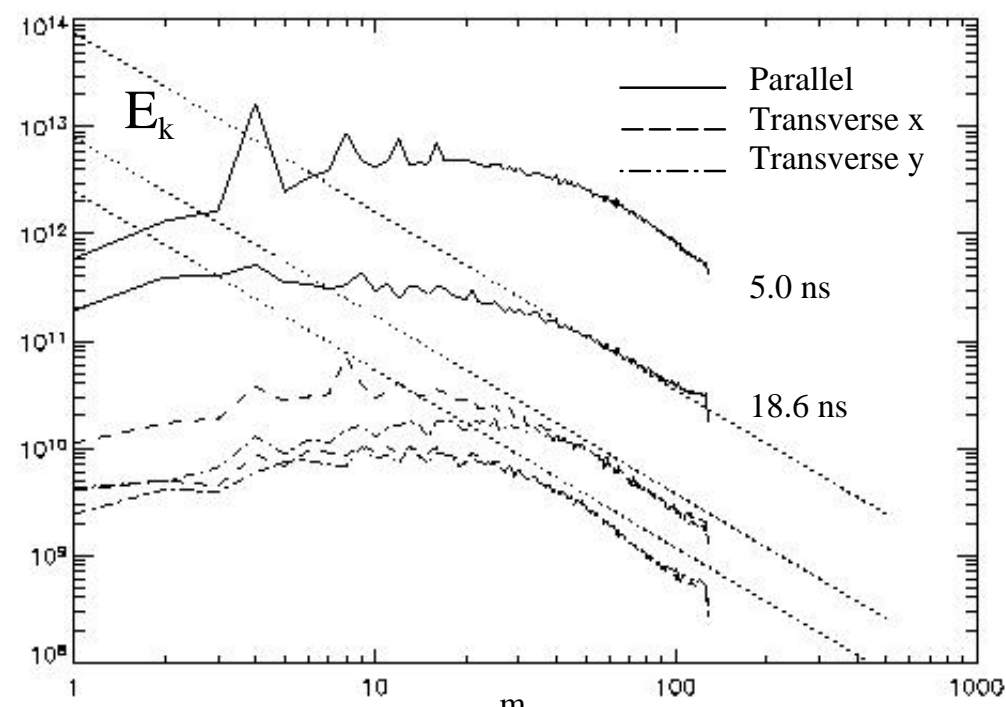

(b)

Figure 16: (a) Density and (b) energy spectra from the 2D m4 plus 3D narrow gaussian shorts calculation. An inertial range is apparent by the time the mixing transition has taken place. The transverse velocity spectrum is nearly isotropic after the mixing transition. 


\section{Conclusions}

When an RT-unstable interface grows to several times its characteristic wavelength, it can undergo a "weak transition" to a disordered, apparently random state. This transition proceeds similarly in 3D and 2D systems with approximately equal nonlinear spike interaction and breakdown thresholds. In the latter case, it can lead to 2D turbulence with an inverse energy cascade due to vortex pairing and merger. A separate inverse cascade in $3 \mathrm{D}$ as well as $2 \mathrm{D}$ systems follows transition to the self-similar regime of bubble competition and merger. In systems that are not spectrally simple, the weak and self-similar transitions are likely to occur nearly simultaneously. In 3D systems, a subsequent mixing transition leads to fullydeveloped classical turbulence if the Reynolds number is high enough. Because the post-transition interface region is more thoroughly mixed in $3 \mathrm{D}$ than in $2 \mathrm{D}$, its growth is more suppressed relative to the single-mode case.

Each of these transitions has time, space, and seed requirements that must be met before it can occur. The time requirement for the mixing transition may be given by a viscous diffusion time for spectrally simple systems, but is more likely to be set by a nonlinearity threshold for strong spike interaction and breakdown when the intial conditions are spectrally complex.

The weak, 2D, and mixing transitions lead to increased small-scale mixing that reduces the instability growth rate at least temporarily. Wherever it occurs, the 
self-similar transition will eventually give accelerated growth if it is allowed to proceed unhindered by system size or drive time constraints. For interfaces that have already undergone a transition to self-similar growth, a subsequent mixing transition can only inhibit future instability growth.

We have considered the effects of decompression and drive decay on 3D turbulence in blast-wave-driven systems. Decompression leads to decay of vorticity in the transverse plane but not in the parallel direction. The Reynolds number in a blast-wave-driven system will continue to increase while the strong-shock approximation is valid, but eventually the instability drive will vanish and any turbulence will be left to decay during the freeze-out stage.

These conclusions are in large part born out by 2D and 3D Raptor calculations of planar blast-wave-driven systems. The 3D calculations have not yet reached the freeze-out stage, but do appear to exhibit a mixing transition despite a grid-resolution Reynolds number only of order a thousand. We have suggested that this is because the dissipation range has been collapsed into only a few cells at the highest wavenumbers, allowing for an effective integral-scale Reynolds number that is at least an order of magnitude larger than the grid-resolution Reynolds number.

In order to more qualitatively test the effect of the code's inherent numerical dissipation on the inertial range, we are currently planning to run a decayingturbulence problem with a known solution. In addition, it would be very useful to do calculations at twice and four times our current resolution to see if the effects of the small scales on the large-scale evolution are altered. Once enough of the inertial range is resolved, we expect that further increases in resolution will be unnecessary. 
Whether or not our current resolution is really sufficient remains for now an open question.

The simulations show sensitive dependence on the initial conditions deep in the nonlinear post-transition phase of the instability growth. Where the low mode 4 is included in the initial conditions, the late-time mix width is much larger. It could be argued that the boundary conditions are affecting the growth at this stage, but that effect should be to inhibit the growth rather than suppress it. Perhaps if the transverse domain could be made arbitrarily large and the drive sustained arbitrarily long, the shorts only and short-on-long systems would begin to look very similar. However, at $20 \mathrm{~ns}$ we are already nearing the freeze-out stage, in which differences in perturbation amplitude will be preserved at later times.

Thus three-dimensional effects do not add anything that will counter the tendency of blast-wave-driven systems to remember some aspects of the initial conditions. For supernova calculations, this means that an understanding of the initial conditions is important for getting that late-time mix width correct. Experiments should include similar initial conditions in order to be truly relevant to supernova. In particular, spectrally simple initial conditions are of limited utility since even the process by which they undergo transition may be different than with more realistic multimode spectra. 
Appendices

\section{Appendix A. CALE simulation of Richtmyer-Meshkov instability experiments at high Mach number}

\section{Introduction}

The Richtmyer-Meshkov (RM) instability occurs when a shock wave crosses an interface between two materials of different densities and/or compressibilities. ${ }^{1,2}$ During the process of refraction, the shock induces a misalignment of density and pressure gradients wherever the normal to the interface is not along the direction of shock propagation. Such a misalignment is a mechanism of vorticity generation. Vorticity is thereby deposited on the interface by the shock in such a way that perturbations on the interface prior to shock refraction will grow in time after passage of the shock. In the simplest model, proposed by Richtmyer ${ }^{1}$ (who in the same paper developed the compressible linear theory of the instability), sinusoidal perturbations grow linearly in time according to

$$
\mathrm{v}=\mathrm{k} \mathrm{a} \mathrm{A}^{*} \mathrm{u}_{\mathrm{i}}
$$

where $\mathrm{k}$ is the perturbation wavenumber, $\mathrm{a}^{*}$ is the post-shock perturbation amplitude, $\mathrm{A}^{*}$ is the post-shock Atwood number, and $\mathrm{u}_{\mathrm{i}}$ is the amount by which the interface velocity increases upon passage of the shock wave (The post-shock interface velocity for an interface initially at rest). 
In his original treatment, Richtmyer considered only the case where the shock moves from a lighter to a heavier fluid. In order to bring Richtmyer's impulsive formula into agreement with experiments in which the shock moves from a heavier to a lighter fluid, Meyer and Blewett later suggested in that case replacing the postshock amplitude with the average of the pre- and post-shock amplitudes. ${ }^{3}$ The Richtmyer and Meyer-Blewett formulae have been successfully applied to the linear phase (in the sense that $\mathrm{k} a<1$ ) of a number of experiments ${ }^{2-4}$ and simulations ${ }^{5,6}$. These have primarily been low Mach number shock tube experiments in which the flow is nearly incompressible from just after shock refraction. In addition, a variety of linear and nonlinear models have been developed and applied, under various conditions, to both single- and multi-mode RM instability evolution. These include the compressible linear theory for the case of reflected rarefaction wave $e^{5,6}$ as well as potential flow, ${ }^{7-9}$ statistical mechanical, ${ }^{7,-12}$ vortex, ${ }^{4,10,15}$ buoyancy-drag, ${ }^{12,14}$ nonlinear perturbative, ${ }^{16,17}$ and phenomenological ${ }^{18}$ models.

Recently, there has been a great deal of interest in high Mach number RM instability effects. ${ }^{19-22}$ In experiments relevant to inertial confinement fusion research, high-powered lasers drive shocks up to $\mathrm{M} \approx 20$ into various targets. ${ }^{23}$ In this regime, compressibility effects can be important late into (or even throughout) the experiment. Many of the models that can correctly predict the instability growth at low Mach number coincide with the Richtmyer of Meyer-Blewett velocity at $t=0,4,7$ $10,12,18$ and therefore must fail when compressibility effects persist long after shock refraction. 
In some heavy to light experiments of moderate and high Mach number, the speed of the transmitted shock relative to the unperturbed interface is not substantially greater, or is even less than the Meyer-Blewett velocity. ${ }^{20,21,24}$ The transmitted shock then remains close to the interface long into the experiment, but the spike tips have not been observed to penetrate ahead into the unshocked material. It is therefore believed that the proximity of the transmitted shock inhibits the instability growth. 21,24,25 However, because growth rate reduction due to large amplitude effects is often potentially also present in these experiments, it has proven difficult to conclusively demonstrate and quantify RM instability growth reduction from shock proximity. ${ }^{26}$

In this paper, we present the results of numerical simulation of single-mode RM experiments conducted on the OMEGA laser at the University of Rochester Laboratory for Laser Energetics. Long into the instability evolution, compressibility effects are apparent and the transmitted shock remains close to the interface.

We begin by briefly describing the experiment, which is detailed more completely in a recent article by Glendinning et $a .^{24} \mathrm{Next}$, we describe the code used to simulate the experiments as well as issues of zoning, convergence, and code-tocode comparison. We demonstrate that the quantity and quality of the data are sufficient to tightly constrain the simulations. A gap between the plastic and foam, resulting from the inability to machine the perturbation into the foam (as described in Section II), visibly affects the interface evolution and so must be included in the simulation. Accurate modeling of the experiment also requires that the simulated target be driven by a source that agrees in detail with the actual laser drive. The results are also shown to be sensitive to the choice of material equation of state 
(EOS). This is especially true for the foam, for which the tabular EOS's considered are shown to be too stiff. Since the proximity of the transmitted shock to the interface is strongly affected by the foam compressibility, our purposes require an accurate foam EOS.

After establishing requirements for the simulations, we proceed to quantify the contribution of the Rayleigh-Taylor (RT) instability and target decompression to the perturbation growth. Both are shown to be insignificant during all but the very latest times. By varying the initial amplitude and considering separate spike and bubble velocity histories, we are able to give a clear demonstration of perturbation growth reduction due to shock proximity as opposed to nonlinear (large initial amplitude) effects. Finally, we close with a brief summary of our results.

\section{Experiment}

In the experiments, an $11 \mathrm{~ns}$ laser pulse nominally of constant intensity $\mathrm{I}=2.6$ $\mathrm{x} 10^{13} \mathrm{~W} / \mathrm{cm}^{2}$ is used in a direct-drive configuration to generate a strong, $\mathrm{M} \approx 10$ shock wave at one end of an $800 \mu \mathrm{m}$-diameter cylindrical target. Three sets of three beams each overlap in time to provide a nearly steady drive. The targets, shown schematically in Fig. 1, consist of a $20 \mu \mathrm{m}$-thick $1.2 \mathrm{~g} / \mathrm{cc}$ polycarbonate ablator, a $1.23 \mathrm{~g} / \mathrm{cc} 170 \mu \mathrm{m}$-thick brominated polystyrene $\left(\mathrm{C}_{50} \mathrm{H}_{48} \mathrm{Br}_{2}\right)$ pusher layer, a $50 \mu \mathrm{m}$ thick polycarbonate layer in which is embedded a $200 \mu \mathrm{m}$-wide brominated polystyrene strip, and a $1 \mathrm{~mm}$-thick $0.1 \mathrm{~g} / \mathrm{cc}$ carbonized resorcinol formaldehyde foam (CRF) payload. The brominated strip acts as a radiographic tracer in order to concentrate x-ray opacity into a limited region near the target axis. A single-mode 


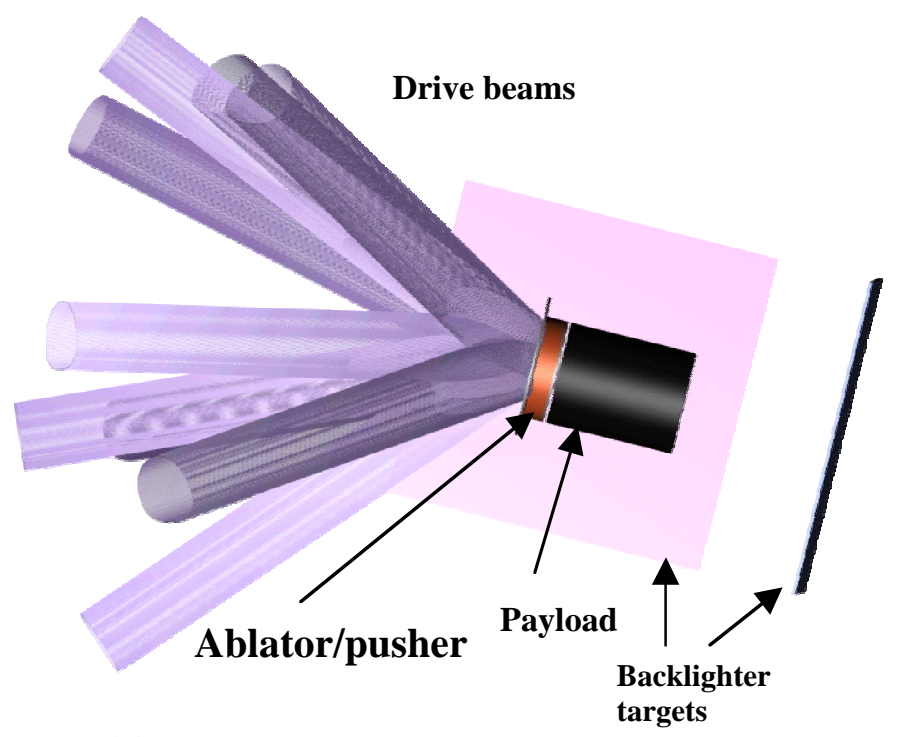

(a) 
perturbation is machined into one end of the pusher, but is not machined into the foam payload. Consequently, there exists a gap (initially air-filled) between the plastic and foam portions of the target. The perturbation wavelength is $150 \mu \mathrm{m}$. Experiments are performed with both large amplitude $(22 \mu \mathrm{m}, \mathrm{ka}=0.92)$ and small amplitude $(7 \mu \mathrm{m}, \mathrm{ka}=0.29)$ perturbations.

The incident shock reaches the interface in about $10 \mathrm{~ns}$, after which x-ray radiographic data is taken over a period of approximately $15 \mathrm{~ns}$. With the passage of the shock, the perturbation undergoes a direct phase inversion. That is, the phase is reversed at the completion of shock refraction. The ratio of the Meyer-Blewett velocity to the speed of the transmitted shock relative to the unperturbed interface is $0.9 \pm 0.4$ for the large amplitude perturbation and $0.4 \pm 0.2$ for the small amplitude perturbation. In the large amplitude case, the spikes of heavier material appear throughout the experiment to be pressed up against the transmitted shock.

\section{Simulation}

We use $\mathrm{CALE}^{27}$ (for C-based Arbitrary Lagrangian Eulerian) to simulate the experiments. CALE is a 2D radiation hydrodynamics code that uses a finitedifferencing method to numerically solve the Euler equations. As an ALE code, it mixes elements of Eulerian and Lagrangian techniques in order to inhibit mesh entanglement.

The target is driven by a time-dependent velocity source extracted from a LANSEX calculation. Additional radiation effects are not included in the simulations 
since the preheat shield, described in Glendinning et al. ${ }^{24}$ effectively eliminates radiation preheat at the interface. The effect of electron conduction is also small, but it is nonetheless included in the simulations.

The initial length of the computational grid is $700 \mu \mathrm{m}$. Its width is $75 \mu \mathrm{m}$, equal to one half the perturbation wavelength. Reflection boundary conditions are specified on the boundaries parallel to the target's symmetry axis, while free boundary conditions are used on the orthogonal boundaries. The pusher density is 1.1 $\mathrm{g} / \mathrm{cc}$ - slightly lower than in the experiments. We have verified that this difference, which is introduced because of details of the EOS tables, has no significant effect on the simulation results. The foam density is $100 \mathrm{mg} / \mathrm{cc}$, as in the experiment. The air gap between plastic and foam is included in the simulations, and its density is set to 1 $\mathrm{mg} / \mathrm{cc}$.

Except where otherwise specified, we use the EOP tables ${ }^{28}$ for the EOS of all three materials. The initial temperature of all materials is set to $25 \mathrm{meV}(290 \mathrm{~K})$. The pressure in the pusher behind the incident shock is $2.4 \mathrm{Mbar}$, and the post-shock pressure at the interface is about $0.5 \mathrm{Mbar}$. In order to study the impact of various EOS models on the instability development, simulations were also performed in which LEOS tables ${ }^{28}$ or perfect gas models were used for one or more of the target materials. The time for an acoustic signal to travel one wavelength in the shocked target is close to the total duration of the experiment: approximately $18 \mathrm{~ns}$ in the plastic and $11 \mathrm{~ns}$ in the foam. 


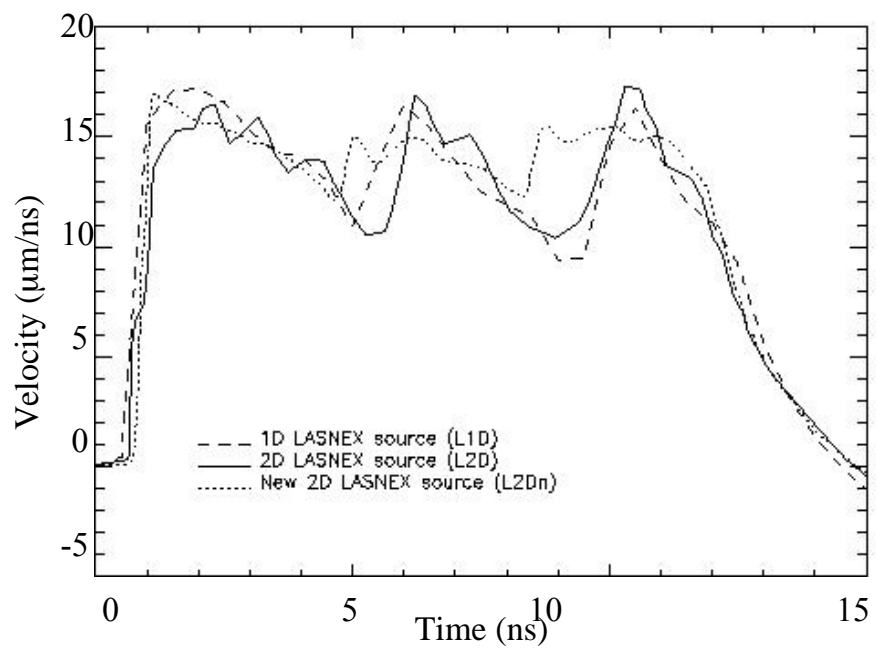

Figure 2: Time-dependent velocity source obtained from 1 and 2D LASNEX simulations and input into CALE as L1D, L2D, L2Dn as described in the text.

\section{A. Velocity drive}

Rather than the constant velocity piston of an idealized RM experiment, the actual target is driven by three sets of drive beams overlapping in time.

Unfortunately, deviations of the real drive from the ideal case have a significant impact on the instability evolution. The simulated laser drive has been continuously been refined as VISAR characterization of the shock trajectory has improved, and the resulting drive progression serves to illustrate the sensitivity of the experiment to these details.

A 1 or $2 \mathrm{D} \mathrm{LASNEX}{ }^{29}$ simulation is used to translate the laser pulse into a time-dependent pusher velocity (called in what follows L1D, L2D, and L2Dn - see Fig. 2). The velocity profile is then input into a CALE simulation as a time-dependent velocity source, where it is used to generate the incident shock wave. The interface 

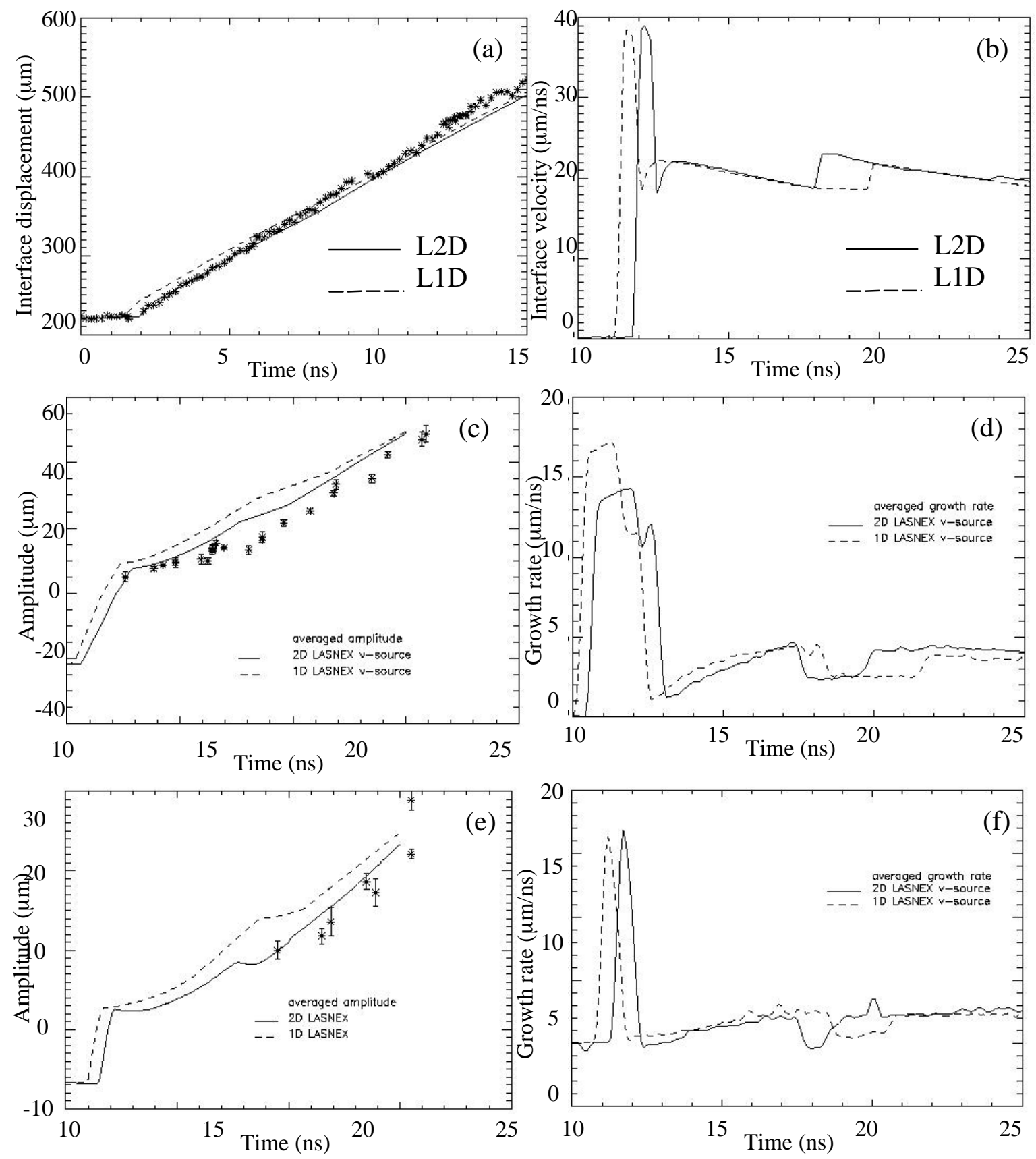

Figure 3: Comparison of simulations driven by velocity source from 1 or 2D LASNEX calculation (L1D and L2D): Interface (a) position and (b) velocity as functions of time. (c) Perturbation amplitude and growth rate for perturbations with initial amplitudes of $22 \mu \mathrm{m}$ [(c) and (d)] and $7 \mu \mathrm{m}$ [(e) and (f)]. Asterisks denote experimental data. 
velocity obtained from a 1D CALE simulation driven by either L1D or L2D is slightly smaller than in the experiment after about 16ns [see Fig. 3(a)].

Despite the subtleness of the differences between L1D and L2D, they have a clear effect on observable features of the instability evolution. By the end of the simulation (at $25 \mathrm{~ns}$ ) the interface driven by L2D has traveled about $15 \mu \mathrm{m}(3 \%)$ further than with L1D. The time-dependent interface velocity [Fig. 3(b)] shows that the temporary reduction in growth rate that occurs between 17 and 22 ns [Figs. 3(d)3(f)] corresponds to the arrival of a second shock at the interface. Investigation of modified velocity sources has demonstrated that this second shock corresponds in turn to the third peak in the velocity source.

The average perturbation amplitude $\mathrm{a}(\mathrm{t})$ is defined as half the distance from bubble to spike tip at time $t$ (ie half the mix width). In simulations of experiments with both large and small amplitude perturbations, the L2D simulation results in an amplitude history that agrees significantly better with the data than do simulations with L1D [see Fig. 3(c),3(e)]. In going from L1D to L2D, a* is reduced from 9.0 to $7.7 \mu \mathrm{m}$ for the $22 \mu \mathrm{m}$ initial amplitude case. The latter is just above the experimental error.

The difference in post-shock amplitude between simulations run with L1D and L2D is not an effect of the absolute value of the pusher velocity. This is demonstrated in Fig. 4, which shows that, in idealized calculations driven by a constant-velocity piston, the post-shock amplitude is insensitive to the piston velocity over a range of several $\mu \mathrm{m} / \mathrm{ns}$ (though the subsequent growth rate is not). For comparison, the average velocities of the L1D and L2D from 1 - $11 \mathrm{~ns}$ are 14.0 and 

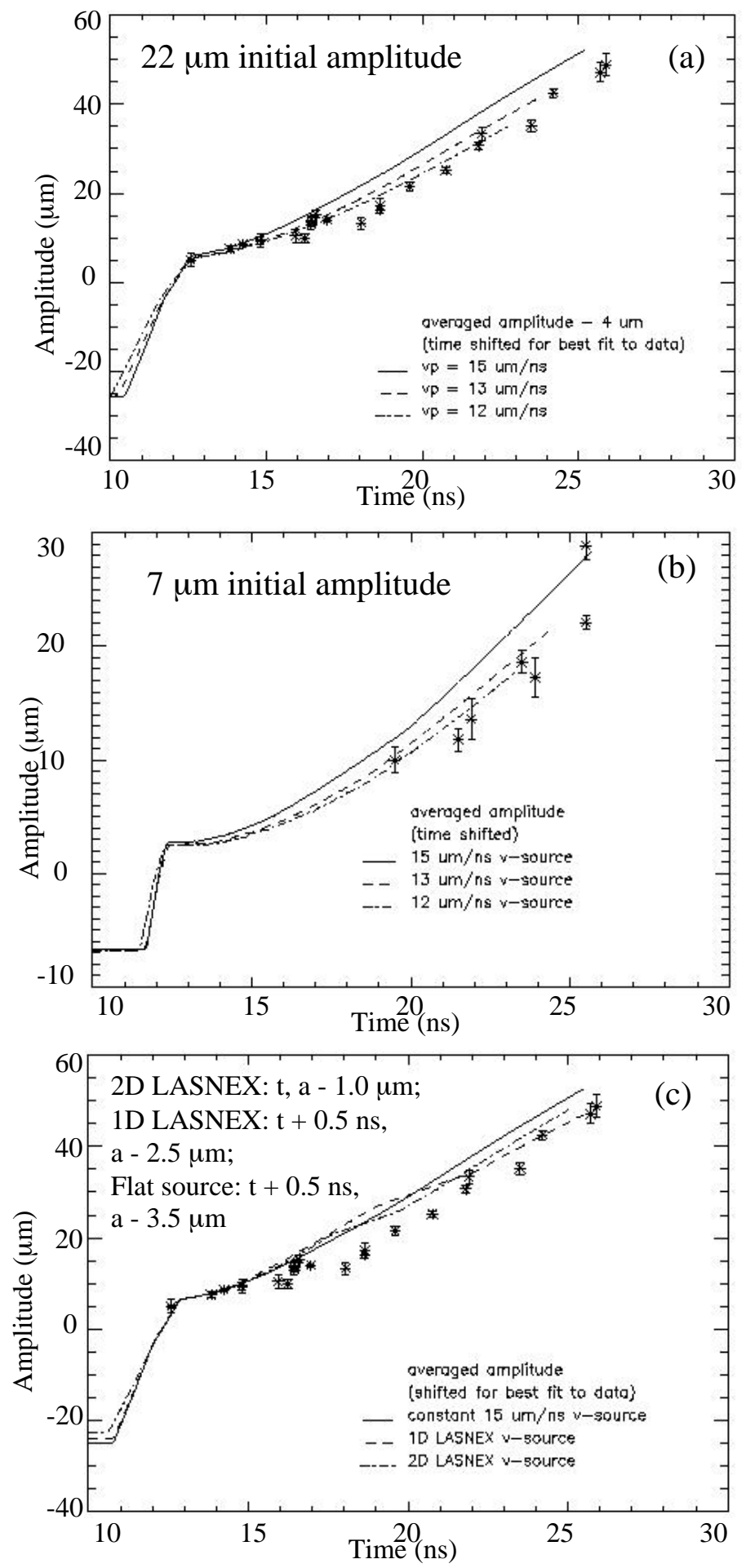

Figure 4: Effect of velocity source details on amplitude history. (a) - (b) The postshock amplitude is insensitive to the pusher velocity over a range of several $\mu \mathrm{m} / \mathrm{ns}$. Note that, in (a) and (b), all three cases have the same absolute amplitude shift. ( c ) Aside from absolute shifts in time and amplitude, the instability develops initially as if driven by a constant velocity (about $12-13 \mu \mathrm{m} / \mathrm{ns}$ ) piston. The histories diverge significantly only upon the arrival of the second shock at the bubble. 

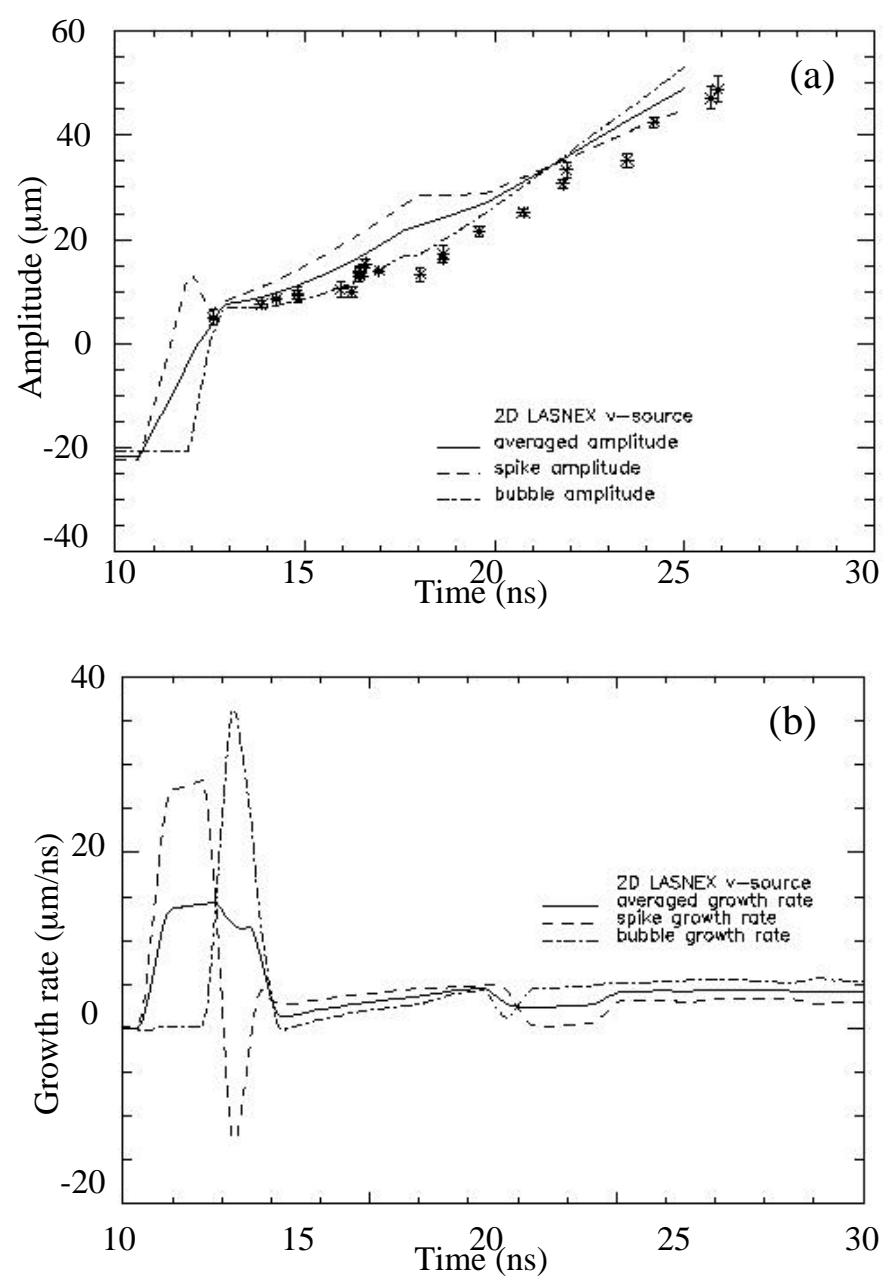

Figure 5: Separate spike and bubble (a) amplitudes and (b) growth rates obtained with L2D.

$13.9 \mu \mathrm{m} / \mathrm{ns}$, respectively. The analogous averages taken from the first to the second peak in the velocity profiles are 15.0 and $14.1 \mu \mathrm{m} / \mathrm{ns}$.

Separate spike and bubble amplitudes and instability growth rates, shown for L2D in Fig. 5, are obtained by subtraction of a reference time-dependent interface position. The reference interface trajectory is obtained from a $1 \mathrm{D}$ simulation including a $22 \mu \mathrm{m}$ gap (equal to the pre-shock perturbation amplitude) separating the plastic and foam regions. The spike growth rate exceeds that of the bubble during the 
first phase of the instability development. Such behavior is predicted by nonlinear theory, but here occurs mainly because the incident shock arrives at the spike about $1.5 \mathrm{~ns}$ before it arrives at the bubble. By the time the second shock arrives at the interface, the bubble velocity has grown to nearly that of the spike. After passage of the second shock, the spike growth rate is reduced while the bubble growth rate is increased such that the new bubble growth rate is larger than the new spike growth rate, and the bubble amplitude soon exceeds that of the spike. By the end of the simulation at $25 \mathrm{~ns}$, the bubble amplitude is about $20 \%$ greater than the spike amplitude.

The reason for the large difference in $\mathrm{a}^{*}$ between L1D and L2D can be understood by considering the individual spike and bubble velocity histories. The initial peak in the spike growth rate provides a measure of the interface velocity immediately after the incident shock breaks out into the air gap. The subsequent peak in the bubble growth rate gives the interface velocity just after breakout of the point on the interface that initially lies at the position of the unperturbed interface. When L1D is used, these two velocities are nearly the same. With L2D, on the other hand, the second is nearly $10 \mu \mathrm{m} / \mathrm{ns}$ greater than the first [Fig. 5(b)]. This sudden change in interface velocity marks the arrival of a shock corresponding to the second peak in the velocity source profile. This is the reason for the relatively large difference in $\mathrm{a}^{*}$ between simulations run with L1D and L2D. With L1D, this second shock overtakes the first just before the interaction begins. Consequently, the shock speed at the beginning of the interaction is about $15 \mu \mathrm{m} / \mathrm{ns}$. When L2D is used, the second shock 
overtakes the first a couple of tenths of a nanosecond after the interaction begins. At the beginning of the interaction, the incident shock velocity is only about $12 \mu \mathrm{m} / \mathrm{ns}$.

The perturbation compression factor in the presence of a gap can be simply expressed by

$$
\frac{a^{*}}{a}=u_{i}\left(\frac{1}{v_{1}}-\frac{1}{u_{g}}\right)
$$

where $u_{i}$ is the interface velocity, $v_{1}$ is the shock speed in the plastic, and $u_{g}$ is the interface velocity in the gap. This reduces to the usual expression in the absence of a gap, when $\mathrm{u}_{\mathrm{g}}=\mathrm{u}_{\mathrm{i}}$. For the reported experimental amplitude and velocity values, this requires $\mathrm{u}_{\mathrm{g}}=28.5 \pm 1.3 \mu \mathrm{m} / \mathrm{ns}$. If the incident shock and interface velocities at the time of interaction are used (rather than their average values), we find $u_{\mathrm{g}}=26.5$ $\mu \mathrm{m} / \mathrm{ns}$. A rough measurement from the data suggests $u_{\mathrm{g}}=20 \pm 10 \mu \mathrm{m} / \mathrm{ns}$. The analogous calculation for the simulations with an EOP EOS gives $\mathrm{u}_{\mathrm{g}}=36.0 \mu \mathrm{m} / \mathrm{ns}$ with L1D and $\mathrm{u}_{\mathrm{g}}=31.8 \mu \mathrm{m} / \mathrm{ns}$ with L2D. The actual values from the two simulations are $34 \pm 1$ and $28 \pm 1 \mu \mathrm{m} / \mathrm{ns}$, respectively. The greater discrepancy between the predicted and observed values of $\mathrm{u}_{\mathrm{g}}$ for L2D is another indication that, with L2D, the velocity of the interface in the gap is not constant over the time in which the gap is being closed.

The shape of the bubble and spike is also affected by the choice of velocity source. With L2D, the neck of the spike is closer to the spike base and is $15 \%$ broader than with L1D or L2Dn (see Fig. 6). The higher narrower necks more closely resemble those observed via side-on radiography (see Fig. 7). In each case, the experimentally observed transmitted shock is more distorted than that seen in the 


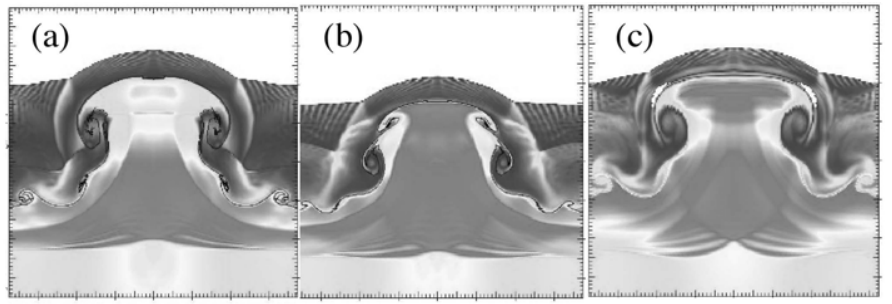

Figure 6: CALE density plots at 20 ns with (a) L1D, (b) L2D, and ( c) L2dn.

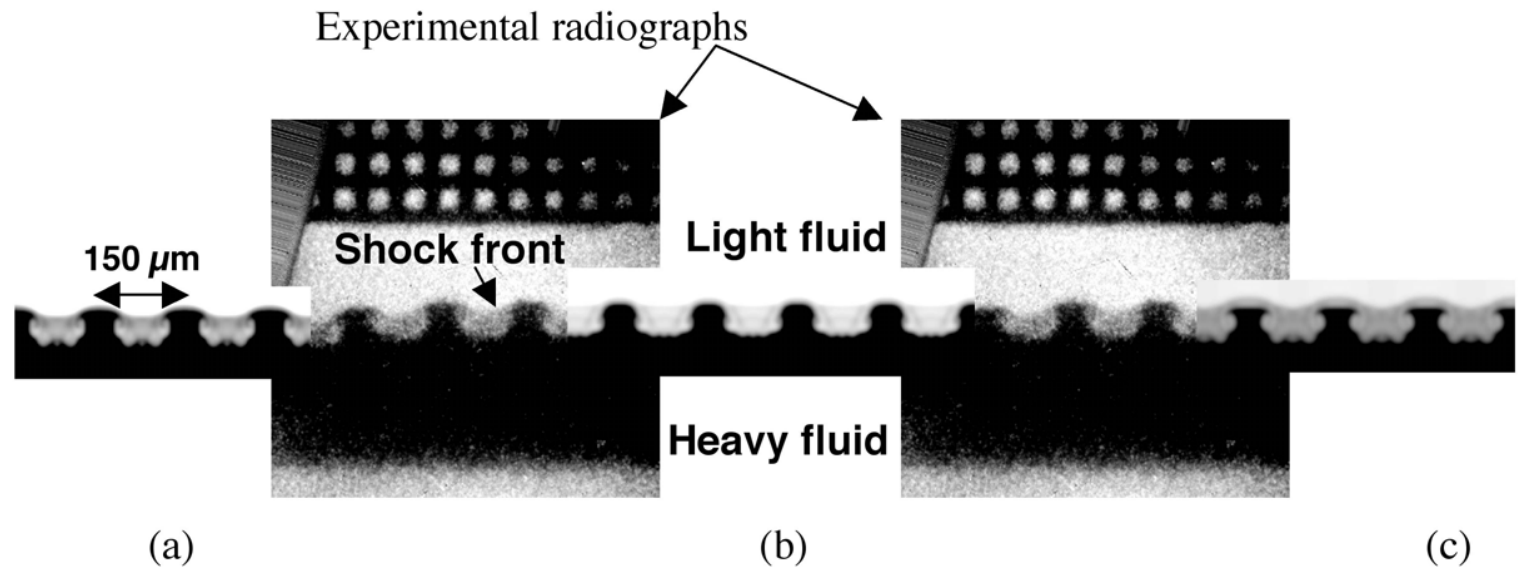

Figure 7: Comparison of experimental radiograph at $20 \mathrm{~ns}$ with CALE simulated radiographs obtained with (a) L1D, (b) L2D, and (c) L2Dn.

simulations, corresponding to an over-prediction of the distance from the bubble to the transmitted shock. The discrepancy, which is more severe with L2D and L2Dn than with L1D, appears to be the only significant experimental quantity more closely predicted by L1D than by the $2 \mathrm{D}$ sources.

Although simulations with either L1D or L2D agree with the general features observed in the experiments, they share in common four discrepancies. These include the over-prediction of $\mathrm{a}^{*}$ and the bubble-shock proximity distance noted above. In addition, CALE predicts a value of $\mathrm{da}(\mathrm{t}) / \mathrm{dt}$ that is too large for as much as 4 of the first $6 \mathrm{~ns}$ of instability development. This is followed by period of 2-3 ns during which the da(t)/dt predicted by CALE is too small. 
Much of the discrepancy between the simulations and experiments can be attributed to an incomplete understanding of the details of the velocity drive. We have already shown that L2D does not produce the large error in a* seen with the L1D. The early time growth rate is also smaller with L2D than with L1D. In the simulations, the reduction and subsequent increase in $\mathrm{da}(\mathrm{t}) / \mathrm{dt}$ in the middle phase of the instability development results from the passage of a shock generated by the third peak in the velocity source profile. A similar phenomenon is suggested by the data, but occurs somewhat earlier than in the simulations. The difference is about $1 \mathrm{~ns}$ for L2D and 2 ns for L1D.

The observed sensitivity of the instability evolution to details of the velocity source has prompted efforts to better characterize the drive and, consequently, the laser pulse shape. Recently improved measurements have produced an improved pulse shape. A 2D LASNEX simulation run with the improved pulse has been run and a new velocity source (L2Dn for L2D new) extracted for input into CALE. Results of CALE simulations run with L2Dn are shown in Fig. 8, where they are compared with L2D results. As expected, the plot of interface velocity shows that the second weak shock arrives at the interface a couple of nanoseconds earlier than predicted by L2D. The resulting amplitude history is in excellent agreement with the data except for a post-shock amplitude that is $5 \mu \mathrm{m}$ too large. The latter fact suggests that L2Dn misses the subtle timing of the arrival of the first weak shock during the shock refraction process.

It is clear from these considerations that the instability evolution is quite sensitive to the details of the velocity drive. The drive L2Dn, obtained from a 2D 

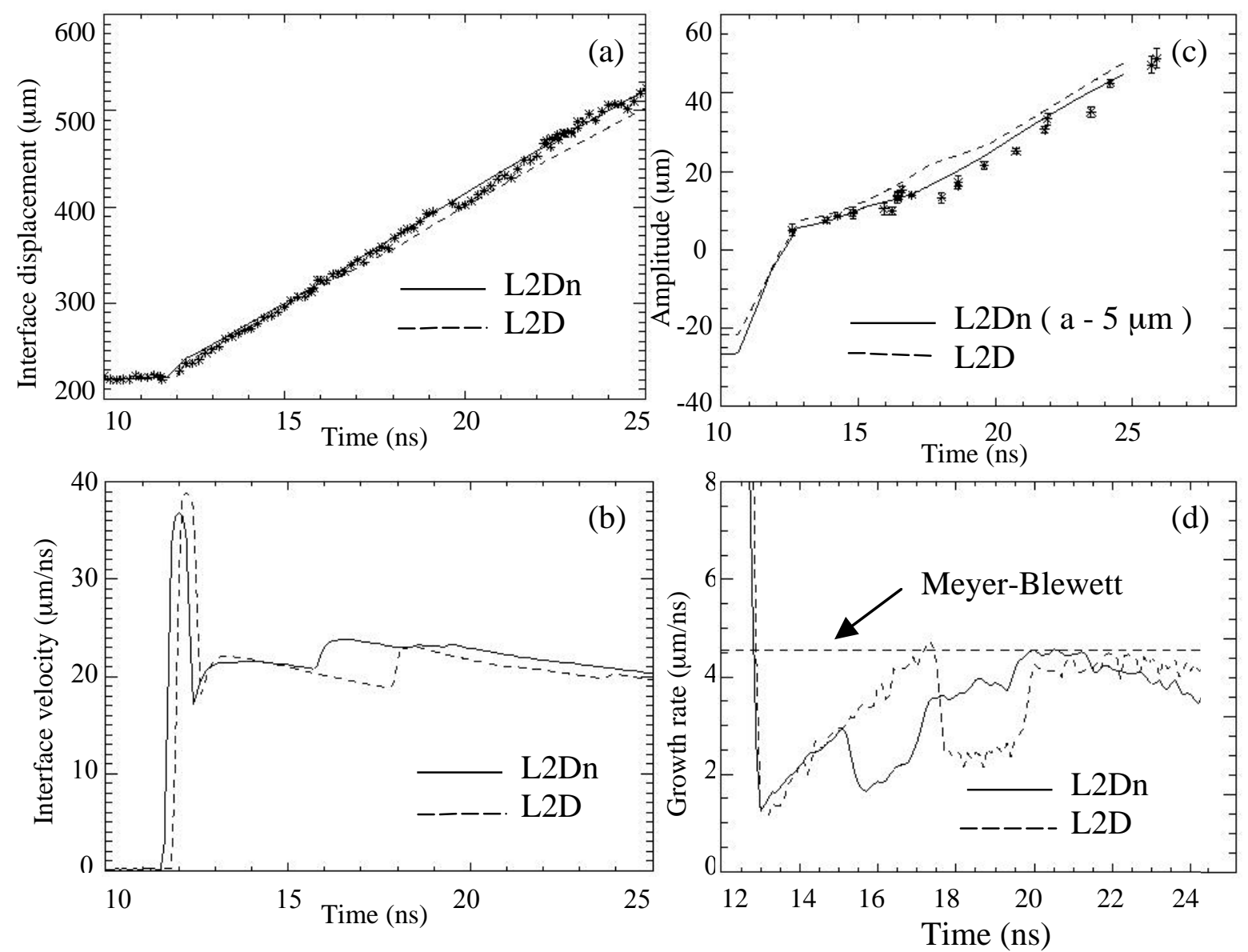

Figure 8: Results with new source (L2Dn) from 2D LASNEX simulation with improved laser pulse shape compared to corresponding results with L2D: (a) Interface position (b) Interface velocity ( c ) Perturbation amplitude $(\mu \mathrm{m})(\mathrm{d})$ perturbation growth rate $(\mu \mathrm{m} / \mathrm{ns})$.

LASNEX simulation well-constrained by experimental measurements, is preferable to either the 1D drive L1D or the less-constrained 2D drive L2D. Despite its overprediction of the post-shock amplitude, L2Dn will typically be employed for subsequent simulations. 

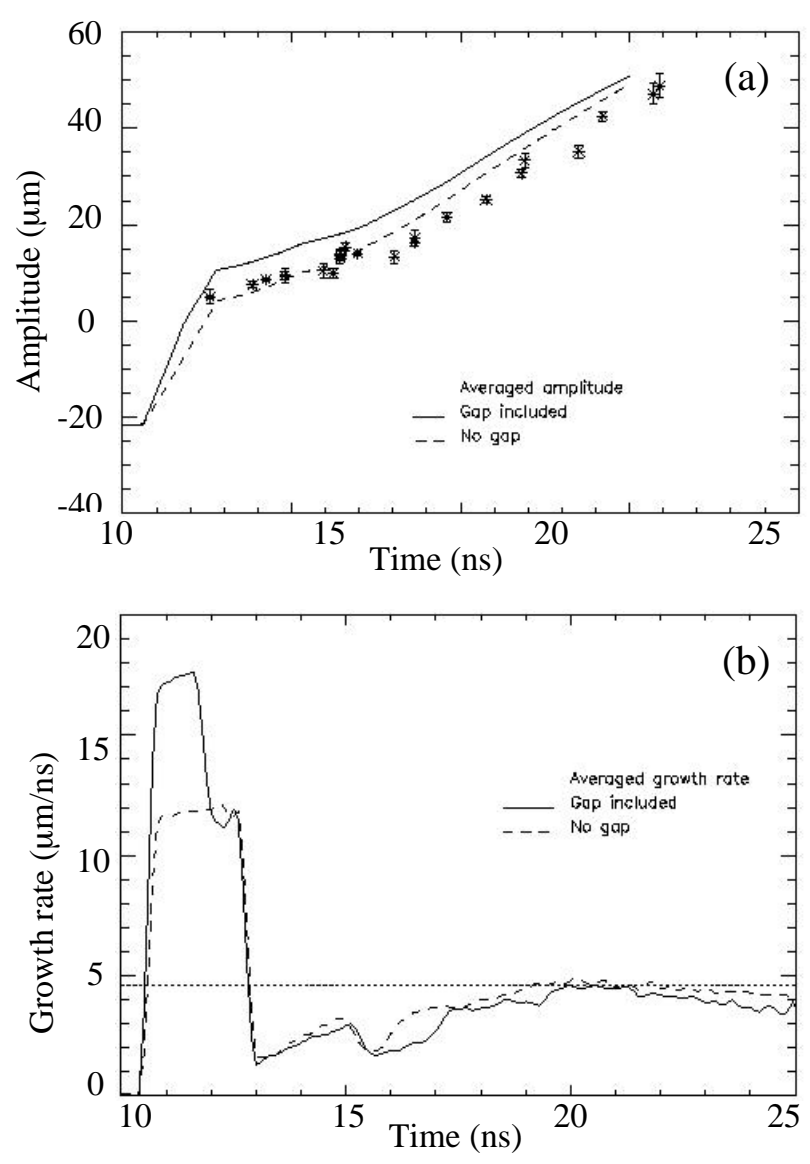

Figure 9: Perturbation amplitude (a) and growth rate (b) with and without air gap between plastic and foam regions.

\section{B. Gap effects}

The perturbation is machined into the plastic pusher but not into the foam payload. As a result, there is a gap between the two materials, which are in contact only at the perturbation peaks. Simulations with and without an air gap show that the presence of the gap increases the early-time spike velocity (and, consequently, the post-shock amplitude - see Fig. 9) and affects the shape of the spike and bubble [see Fig. 10(a)]. However, it does not significantly affect the bubble velocity and the spike 

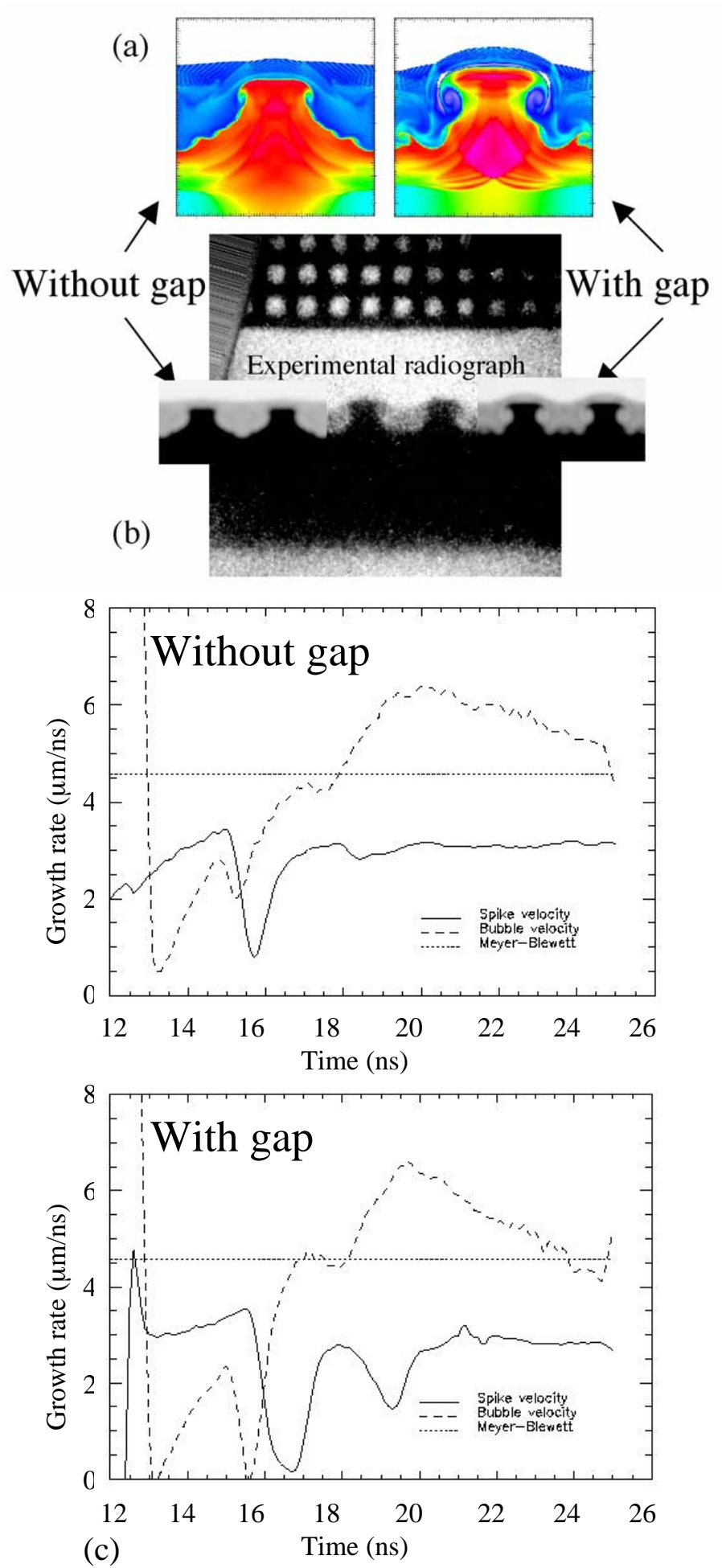

Figure 10: (a) Density plots (at $20 \mathrm{~ns}$ ), (b) radiographs, and (c) spike and bubble velocities for simulations with (right) and without (left) an air gap. The presence of the gap increases the early-time spike velocity (and, consequently, the post-shock amplitude) and affects the shape of the spike and bubble, but does not significantly affect the bubble velocity and the spike saturation velocity. 
saturation velocity [Fig. 10(c)]. Consequently, the effect of the gap is to introduce a vertical shift of about $5 \mu \mathrm{m}$ to the perturbation amplitude history, which it does not otherwise significantly affect.

The post-shock amplitude is most easily affected by the structure of the gap region, to which it is quite sensitive. The foam density is much greater than that specified for the gap. Consequently, when the gap is not included, there is no spike in the interface velocity immediately after the incident shock reaches the interface, and there is a corresponding reduction in $\mathrm{a}^{*}$ that is sufficient to bring it into agreement with the experiment. We have also noted that a lesser reduction in $a^{*}$ results when the gap is partially filled with foam, as might be the case if the foam is partially crushed during target assembly.

The increase in perturbation amplitude caused by the presence of the gap results in a corresponding increase in the distortion (non-planarity) of the transmitted shock. The structure of the perturbation and transmitted shock in simulations with the gap more closely resemble that seen in x-ray radiographs than do simulations in which the gap is not included or is only partially filled with foam [see Fig. 10(b)].

The degree to which the post-shock amplitude, the spike and bubble shape, and the shock planarity are affected by the presence of the gap in the calculations show that the gap must be included if the experiment is to be simulated accurately.

\section{Zoning and convergence}




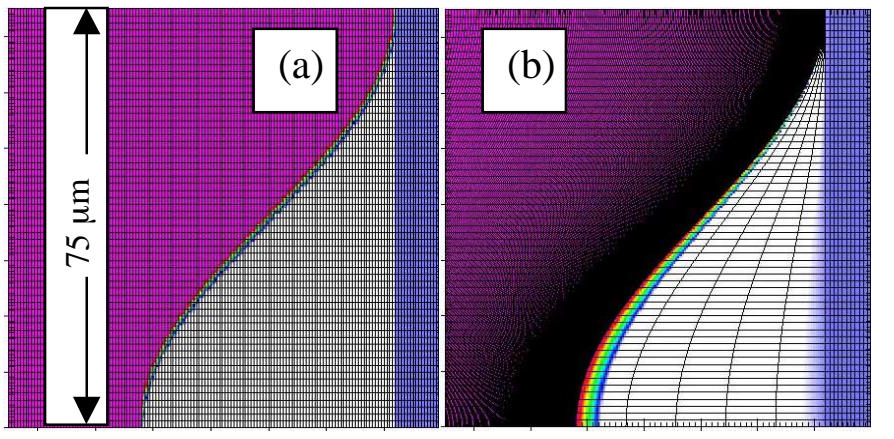

Figure 11: Section of computational domain showing rectangular (a) and conforming (b) computational mesh. An air (white region) gap separates the $\mathrm{CHBr}$ plastic (left) and $\mathrm{CH}$ foam (right) regions.

Two computational mesh configurations were used in the simulations (see Fig. 11). In the first case, a uniform rectangular mesh was specified. The cell dimensions in the rectangular mesh were typically $1.25 \mu \mathrm{m}$ in the direction parallel to the unperturbed interface (corresponding to 120 cells / perturbation wavelength) and $0.5 \mu \mathrm{m}$ in the direction perpendicular to the unperturbed interface. In the second case, the mesh in the foam remained rectangular, with cell dimensions $1.25 \mu \mathrm{m} \times 0.75 \mu \mathrm{m}$. In the pusher, however, a curvilinear mesh was used that conformed to the initial perturbation. A smooth transition between the two configurations was made within the five rows of cells in the gap region.

A zoning study was conducted to determine the optimal configuration and verify convergence in both. In the conforming mesh configuration described above, the computational cells were $0.1 \mu \mathrm{m}$ in the direction perpendicular to the interface at the plastic/void boundary. In a second run, the cells were mass-matched across the plastic/foam boundary with transverse dimensions of $0.01 \mu \mathrm{m}$ in the plastic and 0.1 $\mu \mathrm{m}$ in the foam. This change resulted in a small increase in the post-shock amplitude $\mathrm{a}^{*}$ and early-time instability growth rate $\mathrm{da}(\mathrm{t}) / \mathrm{dt}$. The number of points per 


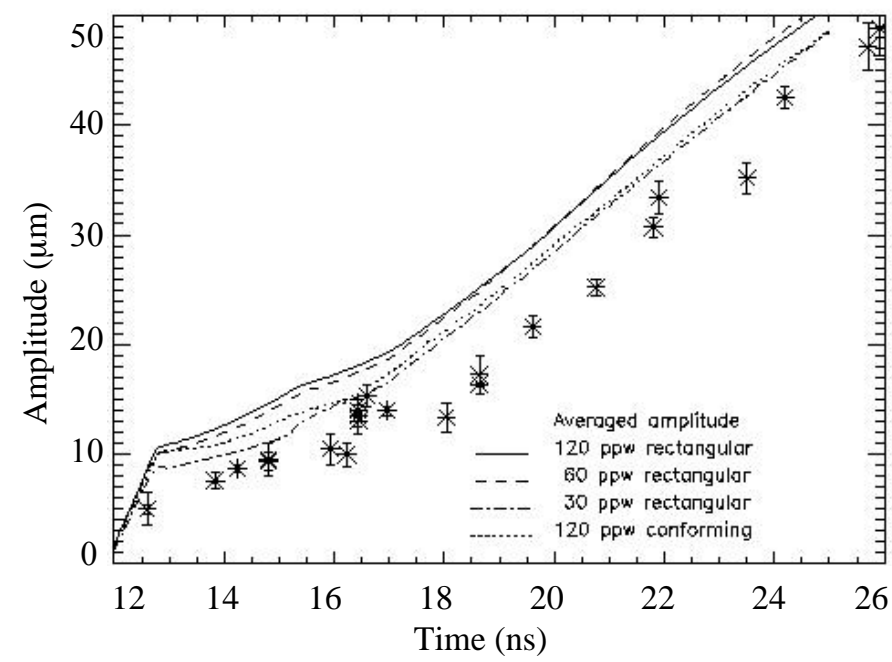

Figure 12: Amplitude resolution study with rectangular and conforming mesh. Results from a simulation with $240 \mathrm{ppw}$ rectangular are identical to those obtained with 120 ppw rectangular, but conforming mesh case converges only at 240 ppw.

perturbation wavelength (ppw) was then increased first to $240(0.75 \mu \mathrm{m} /$ cell $)$ and then to $480(0.375 \mu \mathrm{m} /$ cell $)$. There was no difference in perturbation amplitude history observed between the two latter cases, suggesting convergence at the large scales by 240 ppw.

With the rectangular mesh, clear convergence was seen by $120 \mathrm{ppw}$, as further increase to 240 ppw produced identical results (see Fig. 12). Results obtained from the converged rectangular mesh case and the converged conforming case show reasonably good agreement, with $\mathrm{a}^{*}$ and the early-time da(t)/dt slightly smaller with rectangular mesh than with conforming. The difference in $\mathrm{a}^{*}$ is about $1.5 \mu \mathrm{m}$. Because of its faster convergence properties, the rectangular mesh with cell size $1.25 \times 0.5 \mu \mathrm{m}$ was used in all subsequent simulations.

A one-dimensional study of the effects of cell size for zones mass-matched across the plastic-foam interface was also conducted. Virtually no change in interface 

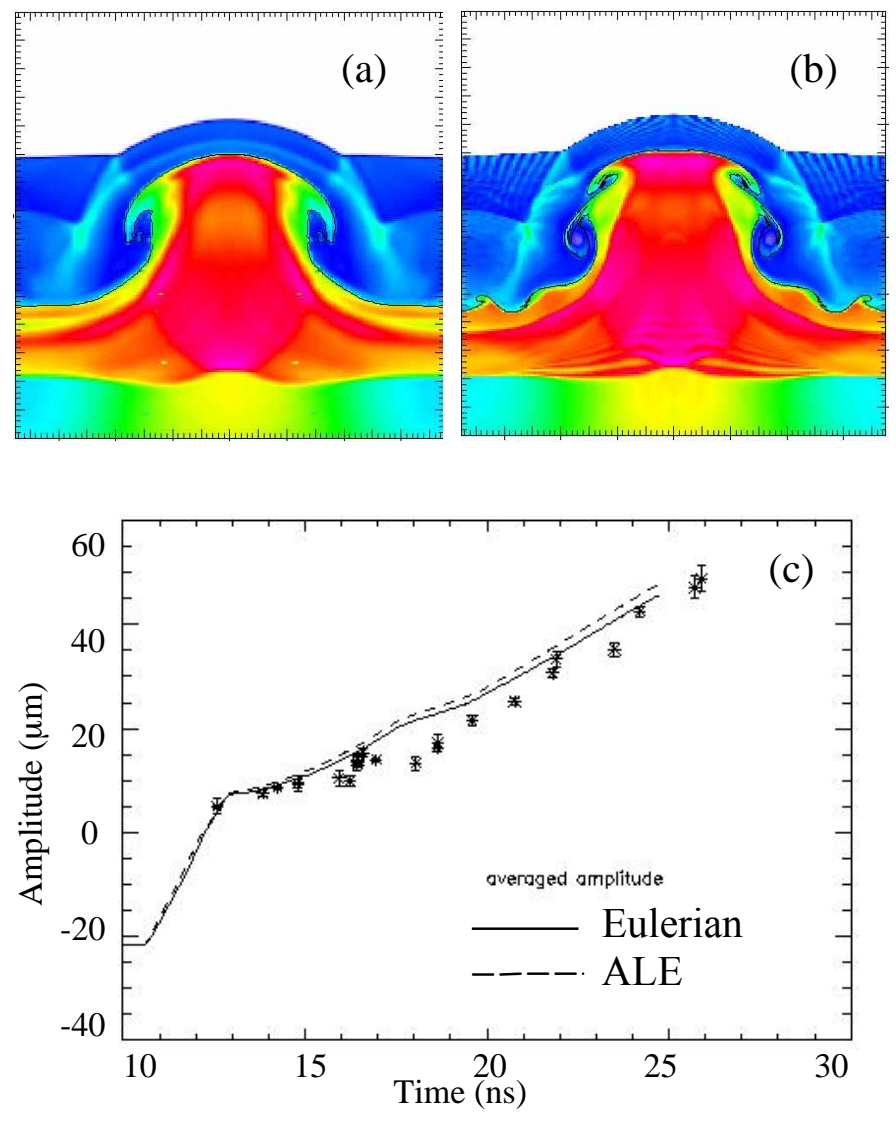

Figure 13: Eulerian / ALE comparison: Density plots at 20 ns from CALE simulations run in (a) Eulerian mode and (b) ALE mode. ( c ) Comparison of amplitude histories with experimental data. The ALE calculation exhibits more Kelvin-Helmholtz rollup at the interface than the Eulerian calculation. However, the differences between the two appear to be below the level of the experimental resolution.

trajectory $\mathrm{z}_{\mathrm{i}}(\mathrm{t})$ was observed when the cell size in the plastic at the plastic-void interface was varied over the range $0.1-0.001 \mu \mathrm{m}$. Over this same range, $\mathrm{z}_{\mathrm{i}}(\mathrm{t})$ was also shown to be insensitive to whether CALE is run in pure Lagrangian or Lagrangian-Eulerian hybrid mode. In most simulations, the ALE feature of CALE was enabled, but an Eulerian calculation was also done for comparison (see Fig. 13). The Eulerian calculation is virtually identical to its ALE counterpart except that it 
exhibits less Kelvin-Helmholtz rollup at the interface. The differences between the two appear to be on scales below the level of the experimental resolution.

\section{Code comparison}

The validity of the CALE setup described above was verified in two different ways. First, CALE simulations of RM experiments reported by Aleshin et al. ${ }^{30}$ were performed. As in the case of OMEGA experiments, a rectangular mesh with $120 \mathrm{ppw}$ was used to simulate one-half perturbation wavelength. The ratio of parallel to transverse cell dimensions was also the same (2.5) in both cases. Results of the CALE simulation were compared with those obtained using another ALE code (HYDRA $\left.{ }^{33}\right)$ and the Adaptive Mesh Refinement (AMR) code RAPTOR ${ }^{34}$ (see Fig. 14). The HYDRA and RAPTOR simulations were run with 512 and 2560 ppw, respectively. Despite the large differences in resolution, all three codes showed excellent agreement on the perturbation amplitude history. Comparison of density plots produced by the two ALE codes showed remarkable agreement on all but the smallest scales.

Since the Aleshin experiments were performed with gases in a shock tube, a second test problem was set up to compare the codes' treatment of the air gap between the plastic and foam. In these simulations, perfect gas EOS's with $\gamma=1.5$ were used for all materials. The pusher density was $1.046 \mathrm{~g} / \mathrm{cc}$, while the densities of the foam and gap region, as well as the perturbation wavelength, were the same as in simulations of the OMEGA experiments. The perturbation amplitude was $22 \mu \mathrm{m}$. The instability was driven by a steady shock created by a piston moving with constant 


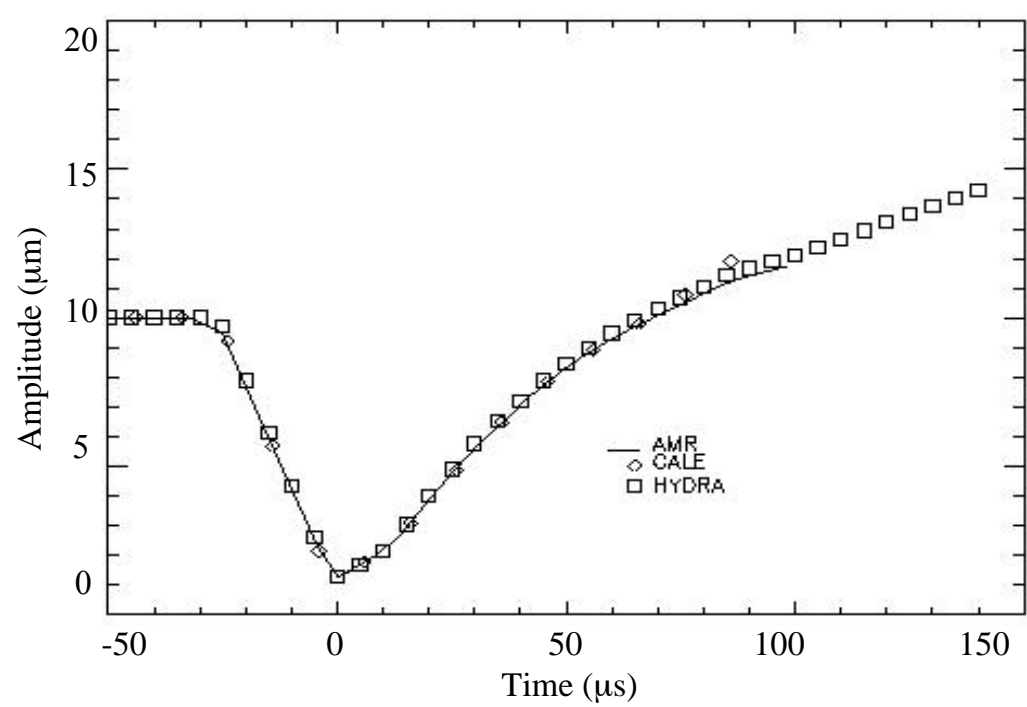

(a)

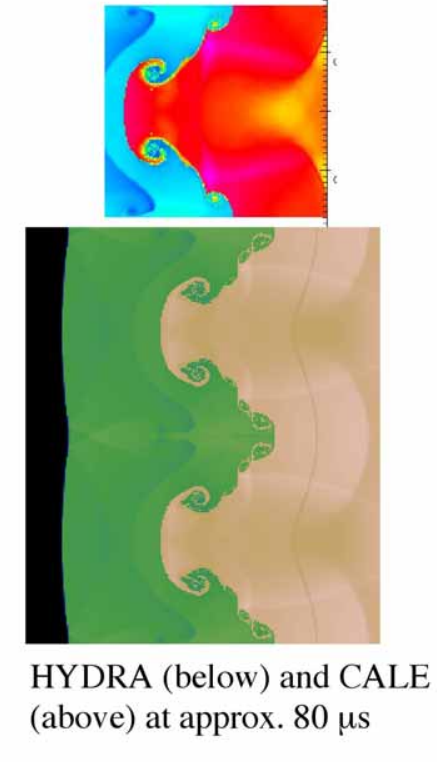

(b)

Figure 14: Code comparison of simulation of Aleshin shock-tube experiment with $\mathrm{M}$ $=4.5$ shock across Xe-Ar interface with $\rho_{\mathrm{Xe}}=2.89 \mathrm{~g} / \mathrm{l}, \rho_{\mathrm{Ar}}=0.89 \mathrm{~g} / \mathrm{l}\left(\rho_{\mathrm{Xe}} / \rho_{\mathrm{Ar}}=\right.$ 0.30), $\mathrm{ka}=1.75$. (a) Amplitude histories obtained using RAPTOR, CALE, and HYDRA. (b) CALE (above) and HYDRA (below) density plots at approximately 80 $\mu \mathrm{s}$. The shock is moving from right to left.

velocity $14.7 \mu \mathrm{m} / \mathrm{ns}$. This piston velocity is nearly equal to the average pusher velocity predicted by LASNEX calculations for the OMEGA experiments. The test problem was run using CALE and RAPTOR. The computational mesh used in the CALE simulation was the same as that used for the Omega experiments. RAPTOR simulations were performed with resolutions of 256 and $512 \mathrm{ppw}$. The simulations were compared up to about $9.5 \mathrm{~ns}$ after the beginning of the shock-interface interaction, beyond which the interface velocities began to decrease (see Figs. 15-16). Over this range, the two codes never differed in their prediction of the perturbation 


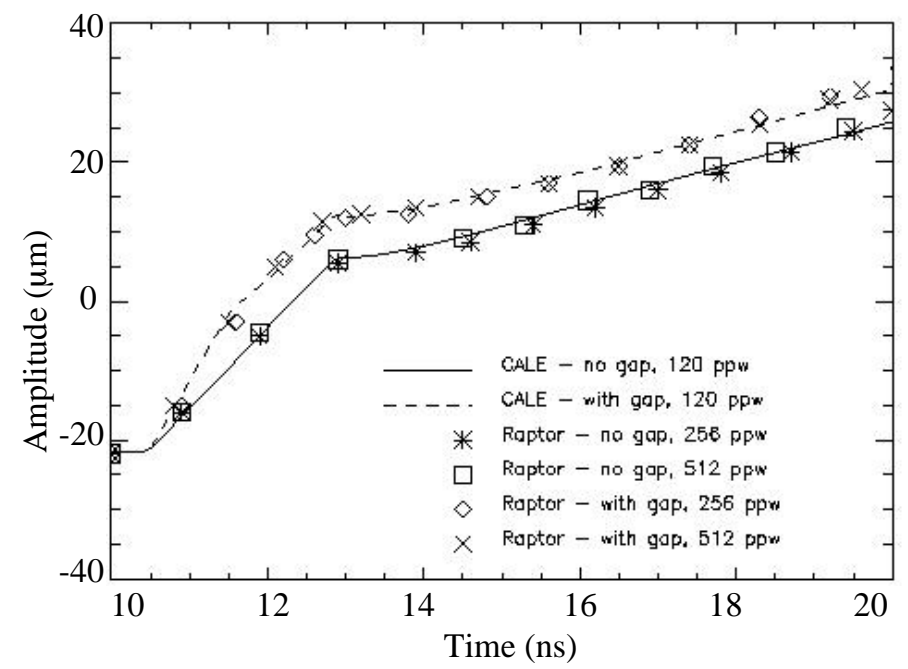

Figure 15: Test problem: Comparison of CALE and RAPTOR simulation amplitude histories with and without an air gap between the two primary materials. Perfect gas EOS for all materials $(\gamma=1.5)$. Constant Piston velocity $=14.7 \mu \mathrm{m} / \mathrm{ns}$. Densities $\rho_{\text {pusher }}=1.046 \mathrm{~g} / \mathrm{cc}, \rho_{\text {payload }}=0.1 \mathrm{~g} / \mathrm{cc}, \rho_{\text {gap }}=0.001 \mathrm{~g} / \mathrm{cc}$.
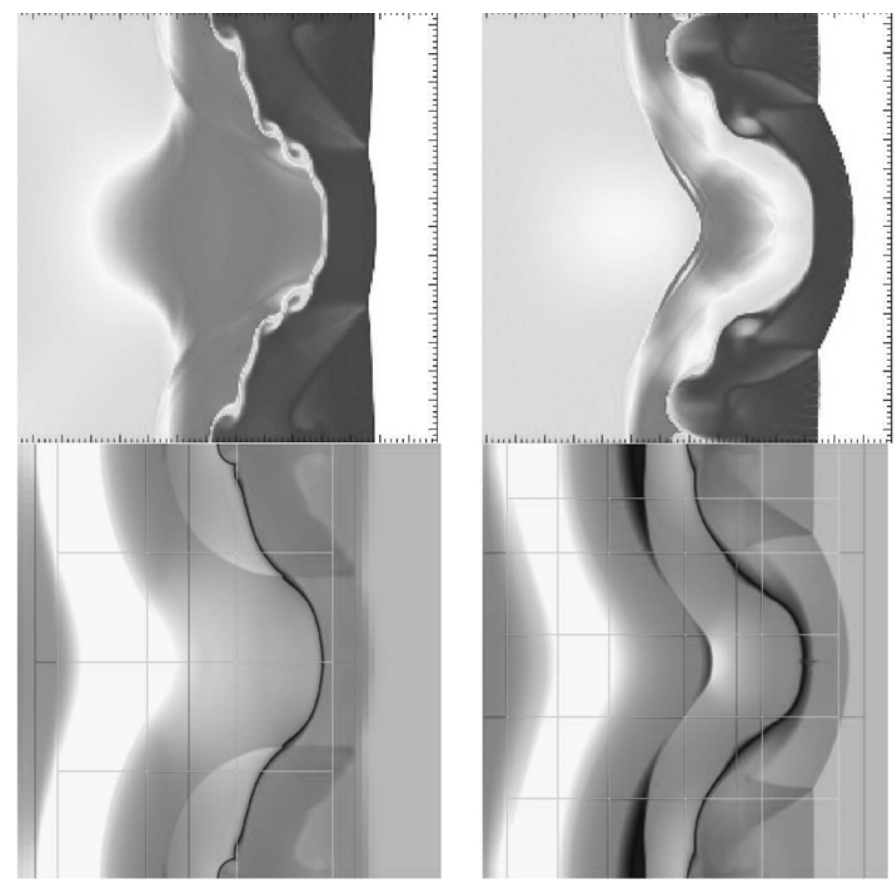

Figure 16: Test problem. Comparison of CALE (above) and RAPTOR (below) simulation density plots at $20 \mu$ s with (right) and without (left) an air gap between the two primary materials. The shock is moving from left to right. 
amplitudes by more than about $3 \mu \mathrm{m}$. The average difference was less than $1 \mu \mathrm{m}$, which is in turn less than the average uncertainty in the OMEGA data.

\section{E. Equation of state}

We have also investigated the extent to which EOS issues could be responsible for the observed discrepancies between the experiments and simulations. Simulations were run in which LEOS tables were used for all materials and where perfect gas models with a range of $\gamma$ 's were used for one or more materials. We considered $\gamma$ over a range from $1.5-2.2$ for the plastic and $1.1-1.5$ for the foam. Some of the EOS results as well as velocity source effects are summarized in Figs. 17-18. The post-shock amplitude depends fairly weakly on the compressibility of the foam (varies by less than $3 \%$ over the range considered). Its dependence on the plastic compressibility is much stronger, but the effective $\gamma_{\text {plastic }}$ would need to be much higher than 2.2 in order to bring $\mathrm{a}^{*}$ into agreement with the data, which seems highly unlikely.

The proximity of the transmitted shock to the interface is particularly sensitive to the foam EOS (and insensitive to drive details), and simulations with both EOP and LEOS consistently over-predict the bubble-shock proximity. In order to match the reported bubble-shock data, the foam compressibility would need to be consistent with about $\gamma_{\text {foam }}=1.3$. The spike-shock and shock distortion data, however, are more consistent with a $\gamma_{\text {foam }}$ of 1.4-1.5. When determined by shock hugoniot curves, the EOP effective $\gamma_{\text {foam }}$ falls in this same range $\left(\gamma_{\text {foam }}^{\text {EOP }}=1.46\right)$, while the LEOS $\gamma_{\text {foam }}$ is somewhat higher still $\left(\gamma_{\text {foam }}{ }^{\text {LEOS }}=1.54\right) .{ }^{28}$

Despite the discrepancy between the time dependence of the experimental and 

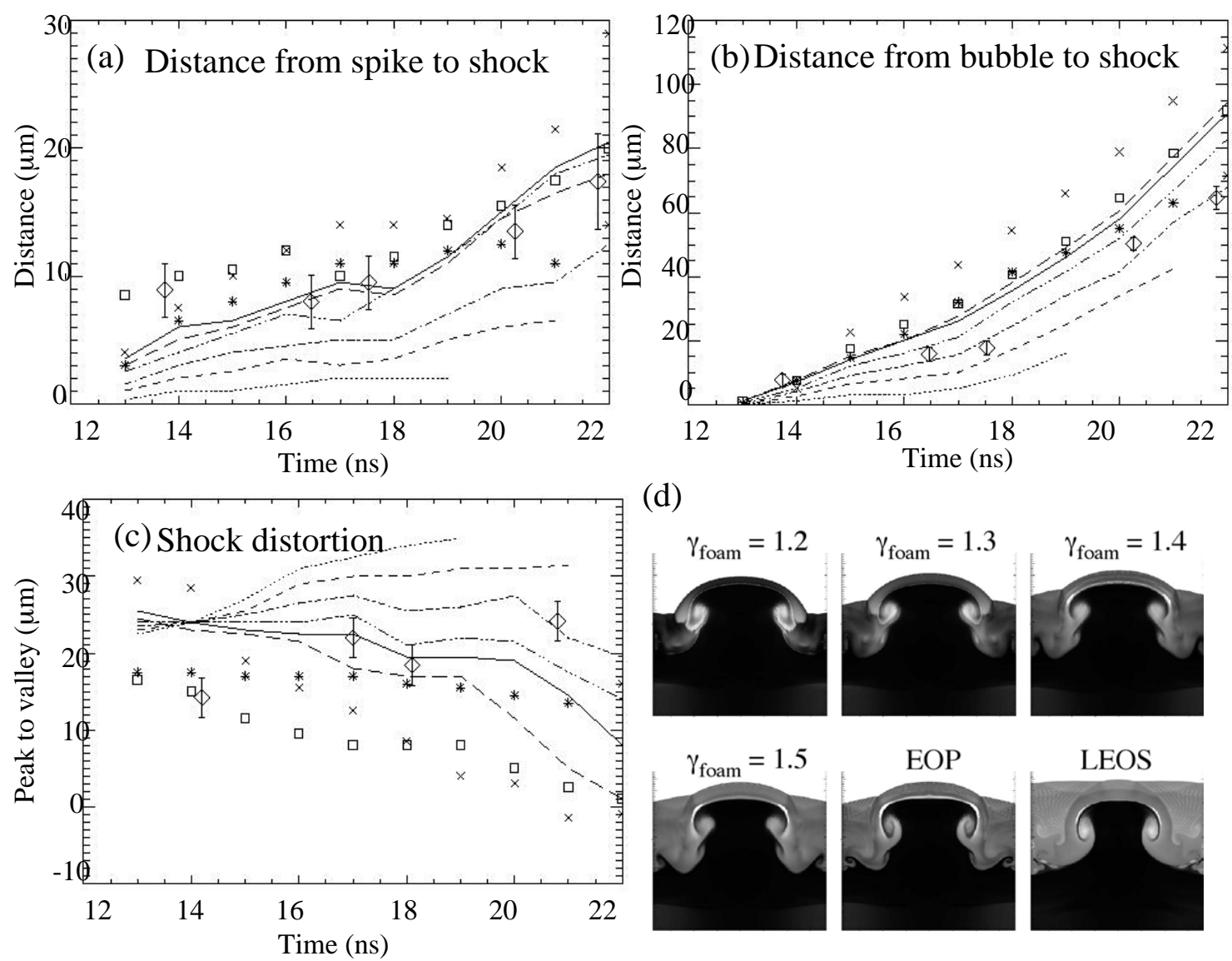

(d)

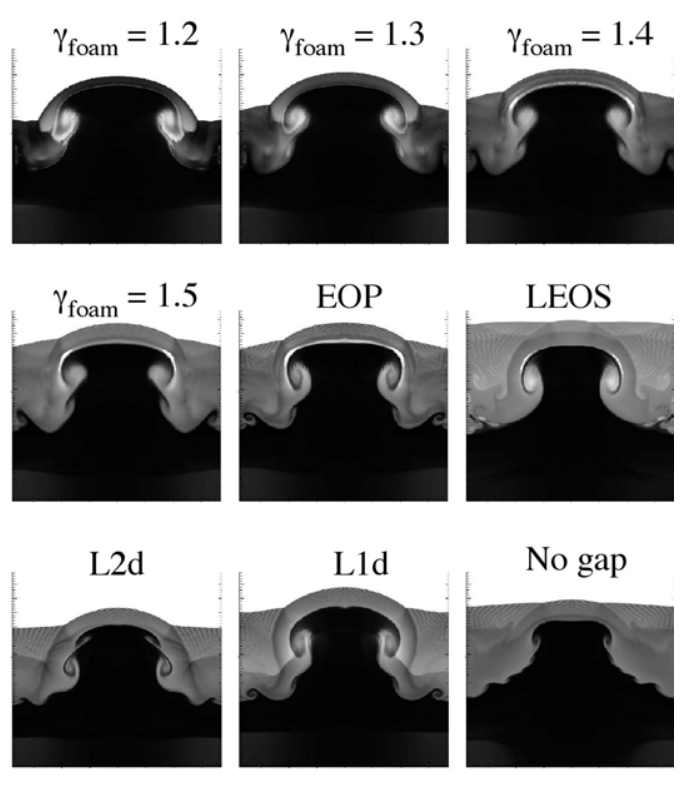

L2dn and EOP EOS unless otherwise specified

$\begin{array}{cllc} & \text { EOP } & \cdots \cdots \cdots \cdots \cdots \cdots \cdots . . & \gamma_{\text {fom }}=1.1 \\ \mathrm{x} & \text { LEOS } & -\cdots--- & \gamma_{\text {fom }}=1.2 \\ \square & \text { no gap } & -\cdots-\cdot- & \gamma_{\text {fom }}=1.3 \\ * & \text { L2D } & -\cdots- & \gamma_{\text {fom }}=1.4 \\ \diamond & \text { Data } & ---- & \gamma_{\text {fom }}=1.5\end{array}$

Figure 17: Effect of equation of state, drive, and gap on transmitted shock proximity and shape. (a) Distance from spike tip to transmitted shock. (b) Distance from bubble tip to transmitted shock. (c) Shock distortion. (d) Numerical radiographs at $20 \mathrm{~ns}$. 

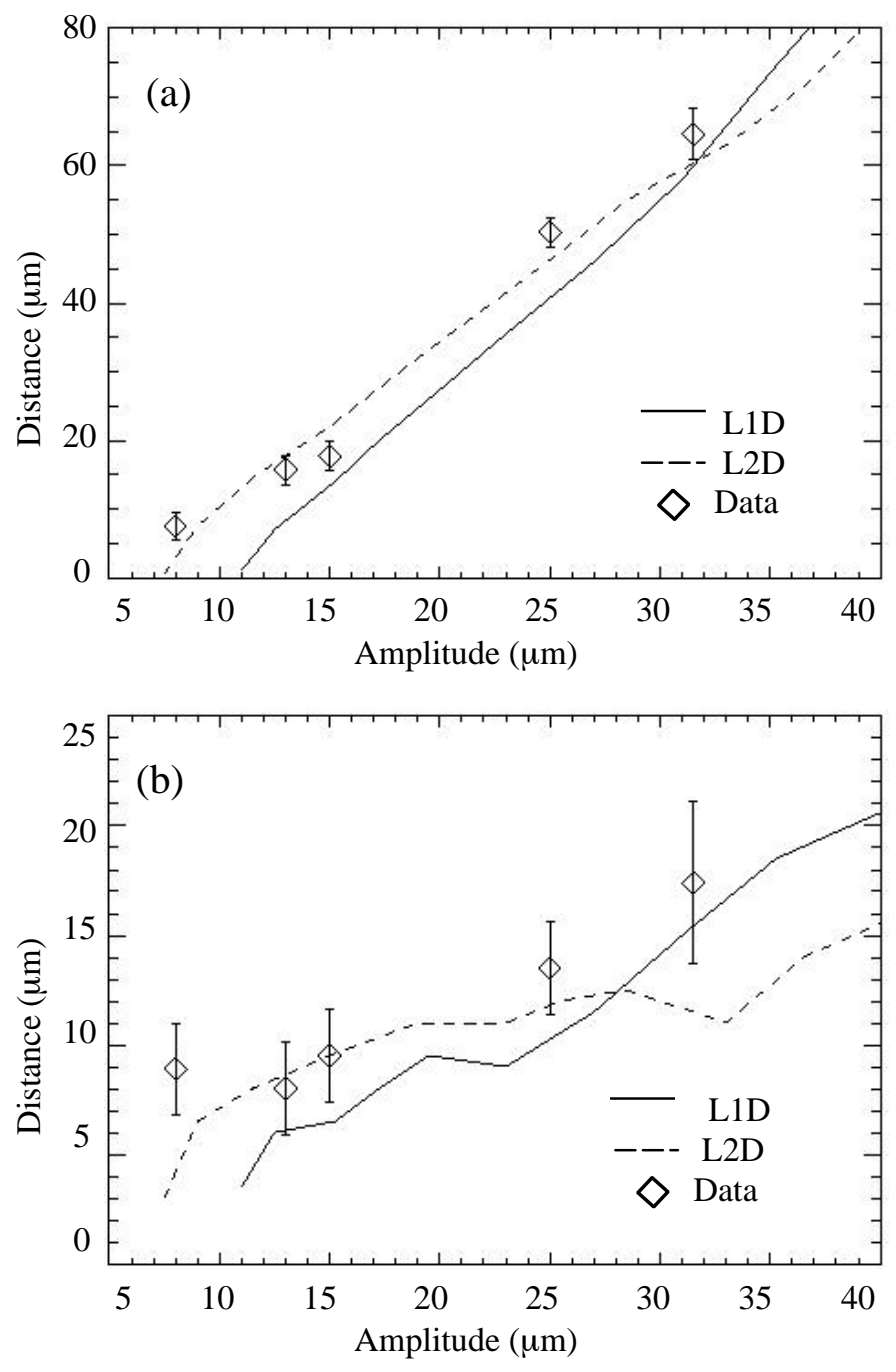

Figure 18: Distance from (a) bubble and (b) spike to transmitted shock vs. perturbation amplitude.

simulation bubble-shock proximity, it appears that the simulation does in fact correctly predict the dependence of the bubble-shock proximity distance on perturbation amplitude (see Fig. 18). This suggests that the error in the bubble-shock proximity results from the over-prediction of the post-shock amplitude rather than an EOS effect. We therefore conclude that the real foam EOS is best represented by the EOP tables and is most consistent with an adiabatic index of 1.4-1.5. In addition, the 
LEOS tables understate considerably the foam compressibility. Consequently, unless otherwise stated, we use the EOP tables all subsequent simulations.

\section{F. Baseline calculation}

Prior to beginning a discussion of the physics issues of interest, we here summarize the above results with a brief description of a baseline calculation. A baseline calculation includes one half of one perturbation wavelength with a transverse resolution of $120 \mathrm{ppw}$. The code's ALE feature is enabled, but the mesh is initially rectangular because a conforming mesh would require greater transverse resolution for convergence. Radiation effects are not included directly, and the target is driven by the planar velocity drive L2Dn, which is extracted from a wellconstrained 2D LASNEX simulation. Electron conduction is included, as is the gap, and the EOP tables are used for all materials. As is apparent in Figs. 7 and 8, such a simulation reproduces well the perturbation amplitude history (except for its $5 \mu \mathrm{m}$ overestimate of the post-shock amplitude) and the interface structure. This agreement depends particularly critically on the inclusion of the gap, the well-constrained velocity drive, and a reasonable EOS.

\section{IV.RT and decompression contribution}

A perfectly uniform laser drive with an intensity that is nearly stationary (but slightly increasing in time) might be expected to correspond to a perfectly steady (ie constant velocity) piston, thereby driving a steady shock into the target. But any real laser pulse corresponds instead to a time-dependent velocity source - at best driving 
into the target a primary shock followed by a series of weak shock and rarefaction waves. In our experiments, there is a local intensity maximum associated with each set of drive beams. The resulting velocity source (see Fig. 2) consists of three peaks superimposed on a constant velocity plateau. The first peak drives the primary shock, and is followed by a small rarefaction wave. The second peak drives a second, weak shock, which overtakes the first immediately before or during shock refraction at the interface. A second weak shock, corresponding to the third peak in the velocity source, reaches the interface several nanoseconds after shock refraction. It too is followed by a weak rarefaction wave followed in turn by a stronger rarefaction wave. The arrival at the interface of the stronger rarefaction marks the end of the period of quasi-constant interface velocity, and consequently the end of the RM experiment. Though nearly constant from the time of arrival of the primary shock at 12 ns to that of the primary rarefaction at about $25 \mathrm{~ns}$, the interface velocity is actually continually decreasing throughout the experiment except during transmission of shocks. As a result of the weak rarefactions, the interface is weakly unstable to the RT instability $^{33,34}$ throughout most of the experiment. In the simulations, the deceleration rate ranges between about 0.4 and $1.0 \mu \mathrm{m} / \mathrm{ns}^{2}$, with its precise behavior depending on the choice of velocity drive. For L2D (the deceleration profile with L2Dn is more complicated), it begins at $1.0 \mu \mathrm{m} / \mathrm{ns}^{2}$ immediately after transmission of the primary shock and decreases continuously to about $0.5 \mu \mathrm{m} / \mathrm{ns}^{2}$ at $25 \mathrm{~ns}$ (Because of its simpler deceleration profile, L2D rather than L2Dn is used in this section). This corresponds to a classical RT growth time, given by $\tau_{\mathrm{RT}}=\left(\mathrm{kg} \mathrm{A}^{*}\right)^{-1 / 2}$, of $7-9 \mathrm{~ns}$, where the postshock Atwood number $\mathrm{A}^{*}=0.54$. With an experiment of duration fewer than $2 \mathrm{RT}$ 
growth times, RT effects will be secondary to RM effects throughout the experiment and utterly insignificant early on, but might become noticeable at late times.

As the target material decompresses in the weak rarefaction fans, geometrical stretching produces additional perturbation growth. Points separated by a distance $\Delta \mathrm{z}$ along the symmetry axis move away from one another with a velocity that increases monotonically with $\Delta \mathrm{z}$ in the rarefaction fan.

In order to quantify the contribution of RT and decompression effects to perturbation growth in the experiment, an idealized set of four simulations was performed. These simulations were driven by constant velocity "pistons" of varying duration (see Fig. 19). The piston velocity is $14 \mu \mathrm{m} / \mathrm{ns}$ - chosen to match the experiment. The longest, a $15 \mathrm{~ns}$ pulse, provides a constant interface velocity throughout the simulation. As it is consequently free from RT and decompression effects, this will be referred to as the pure RM case. Shorter pulses of 10 and $8 \mathrm{~ns}$ yield a period of constant interface velocity followed by a deceleration phase. With a $6 \mathrm{~ns}$ pulse, the rarefaction reaches the interface at about the same time as does the incident shock, so that RT and decompression effects are present throughout. The interface deceleration in these simulations is in the same range as that observed in simulations of the actual experiment. The $6 \mathrm{~ns}$ pulse produces an acceleration profile that is similar to, but somewhat lower than that obtained with L2D.

In the small initial amplitude pure RM case [Fig. 20(a)-20(b)], the results are mostly typical of the classical RM instability. The spike and bubble velocities begin at zero, climb up to a maximum value that is well predicted by the Meyer-Blewett impulsive model, and then fall off asymptotically as $1 /$ t. The spike and bubble 

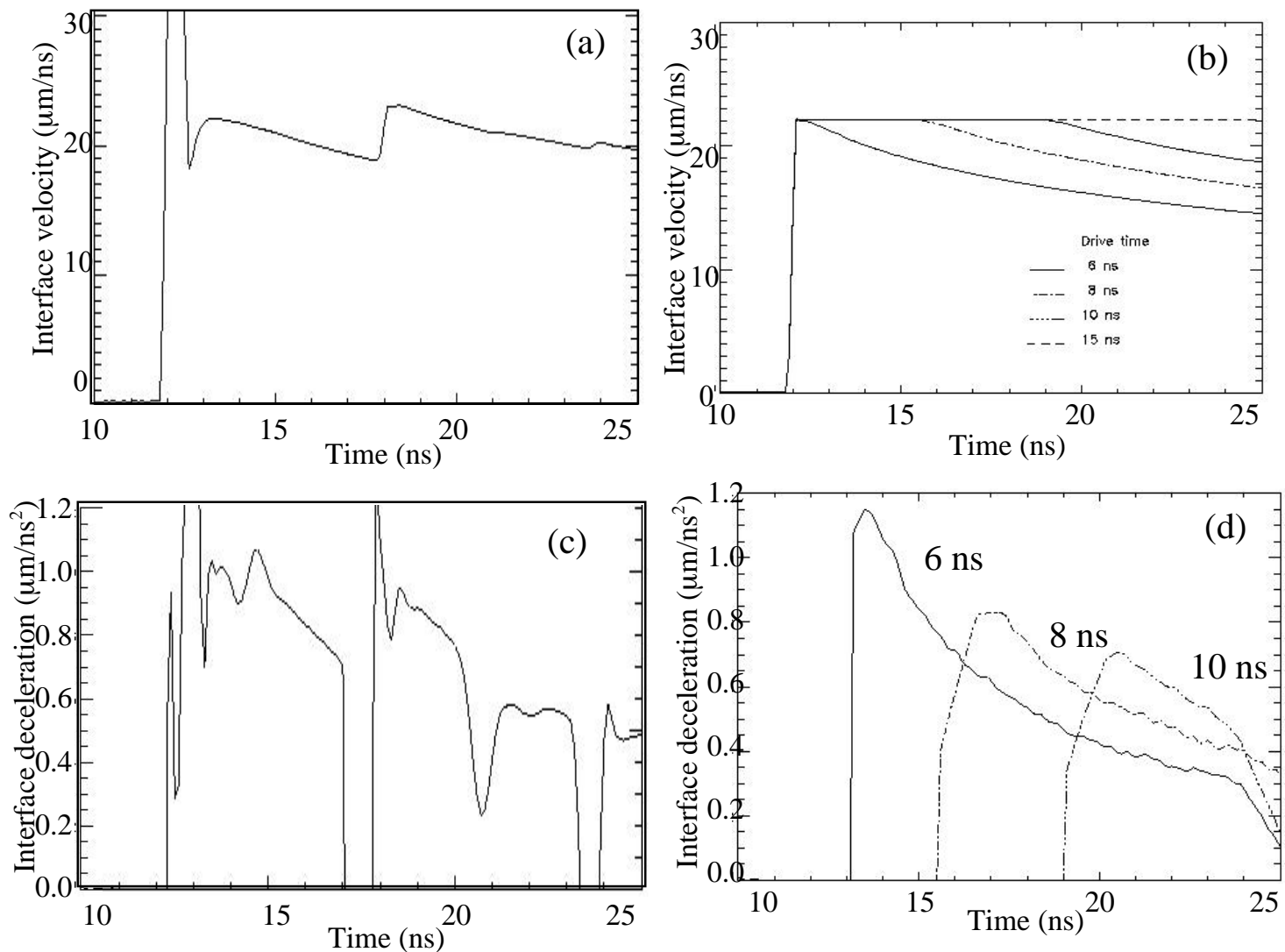

Figure 19: RT / decompression effects: Interface velocity ( $\mu \mathrm{m} / \mathrm{ns})$ obtained (a) with real drive (L2D) and (b) with a series of constant velocity $(14 \mu \mathrm{m} / \mathrm{ns})$ sources of varying duration. Interface acceleration $\left(\mu \mathrm{m} / \mathrm{ns}^{2}\right)$ obtained (c) with real drive and (d) with constant-velocity series. In all cases, the gap is not included. Except in the case of the 15 ns flat drive (pure RM case), the interface velocity is not perfectly constant. During the deceleration phase, the perturbation is RT unstable.

velocities are nearly equal throughout, with the spike velocity perhaps slightly higher than that of the bubble as predicted by nonlinear models. ${ }^{8,9,16-18}$ When the target is driven by the 6 ns pulse, the spike and bubble velocities asymptote to a constant value [Fig. 20(c)]. This is consistent with classical nonlinear RT evolution, where kinematic drag allows the spike and bubble to reach terminal velocities. The difference in averaged amplitude between the two cases does not reach $2 \mu \mathrm{m}$ until about $21 \mathrm{~ns}$, and is only $6 \mu \mathrm{m}$ at the end of the simulation. Still, the difference is sufficient to conclude, based 

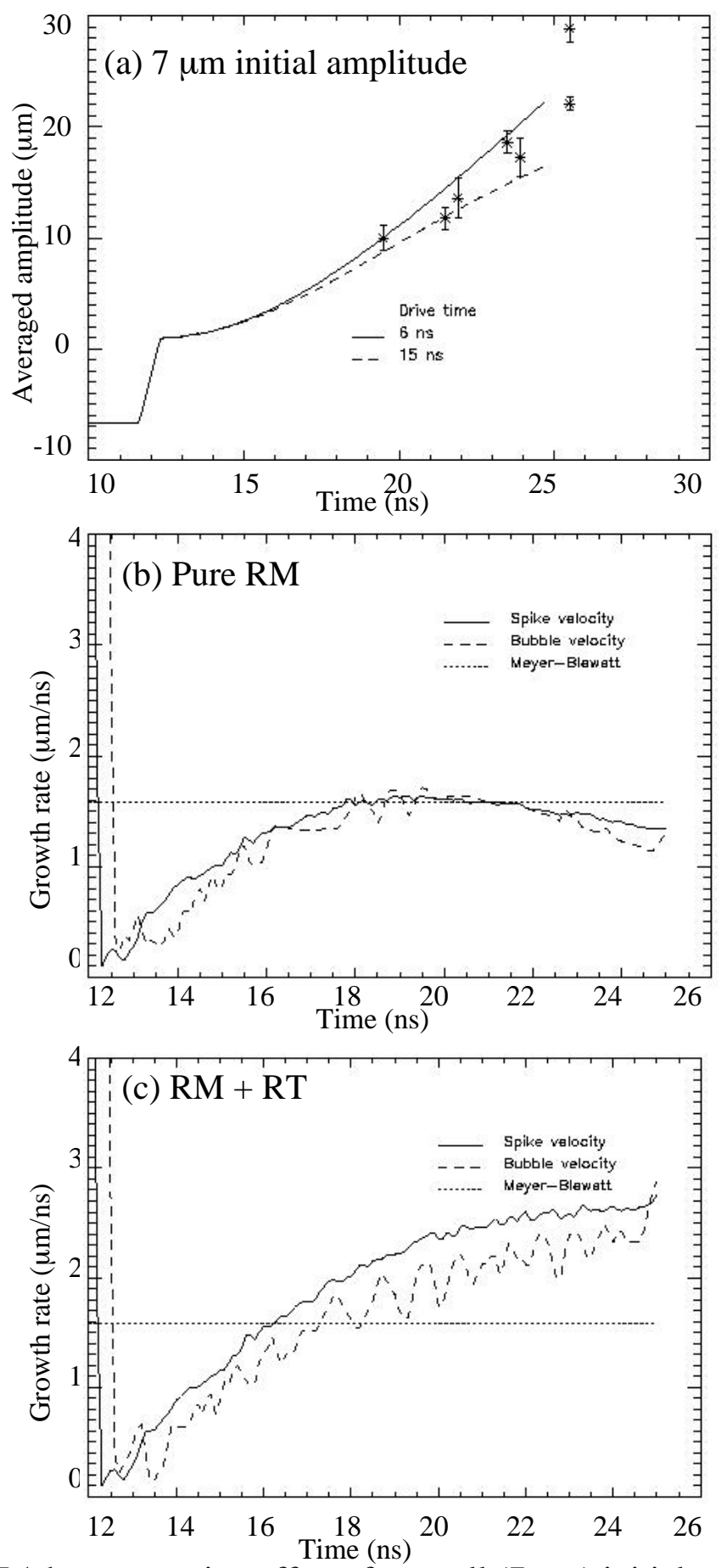

Figure 20: RT / decompression effects for small $(7 \mu \mathrm{m})$ initial amplitude driven by constant velocity (14 $\mu \mathrm{m} / \mathrm{ns})$ drive. (a) Perturbation amplitude. RT / decompression effects are initially small, but become significant at about the time the peak growth rate is attained in the pure RM case. (b) Pure RM case (15 ns drive time) spike and bubble velocities. The asymptotic spike and bubble velocities decay approximately as 1/t. (c) RM + RT case (6 ns drive time). Interface deceleration yields an RT / decompression contribution and a near constant saturation velocity. In both (b) and (c), the spike and bubble velocities remain nearly equal throughout, with the spike velocity perhaps slightly higher than that of the bubble. 
on the data, that RT and expansion effects determine the asymptotic nature of the instability in the experiment. While these effects are less significant, even at late times, than in previous laser-driven experiments intended to study the RM instability, ours remains a true RM experiment only until shortly after 20 ns.

With an initial amplitude of $22 \mu \mathrm{m}$, the bubble behavior mirrors that seen in the $7 \mu \mathrm{m}$ initial amplitude case. Again, its velocity in the pure RM case [Fig. 21(b)] grows from zero up to a maximum at the Meyer-Blewett velocity and then falls off approximately as $1 /$ t. The spike growth, on the other hand, is inhibited by the proximity of the transmitted shock, as will be discussed later. In the 8 and $10 \mathrm{~ns}$ drive pulse cases [Figs. 21(c)-21(d)], the point at which deceleration begins and RT/decompression effects set in is clearly seen in plots of the spike and bubble velocities. Here too, the spike velocity falls clearly below that of the bubble within a few nanoseconds after shock refraction. Finally, we note that there is very little variation in averaged amplitude between the 6,8 , and 10 ns cases [Fig. 21(a)]. At early times, the perturbation growth is dominated by the RM instability - the same for all cases. At late times, the interface deceleration values are very similar in all three cases, and therefore so too are the terminal velocities of the spike and bubble (Actually, the late-time deceleration with the 6 ns pulse is slightly lower than in the 8 or $10 \mathrm{~ns}$ cases, resulting in a late-time decay of its asymptotic velocity that is slow but discernable).

In order to estimate the relative contributions of RT and the decompression effect, the unperturbed fluid velocities at the time-dependant spike and bubble 

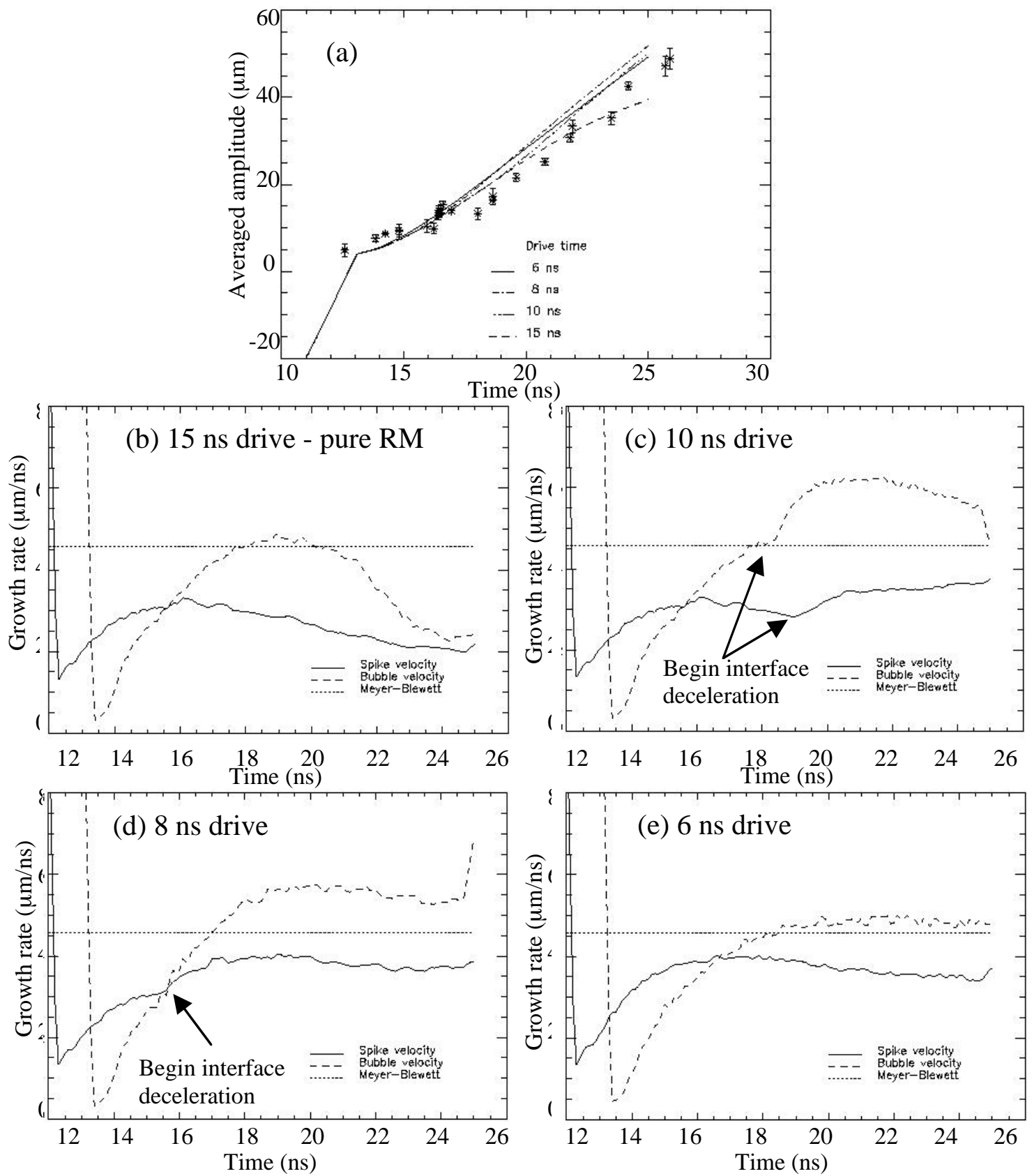

Figure 21: RT / decompression effects for large $(22 \mu \mathrm{m})$ initial amplitude driven by constant velocity $(14 \mu \mathrm{m} / \mathrm{ns})$ drive. (a) Perturbation amplitude. RT / decompression effects are initially small, but become significant at about the time the peak growth rate is attained in the pure RM case. (b) Pure RM case (15 ns drive time) spike and bubble velocities. The asymptotic bubble velocity decays approximately as $1 / \mathrm{t}$. ( c ) 10 ns drive time. (d) 8 ns drive time. (e) 6 ns drive time. Except with 15 ns drive (pure RM case), interface deceleration yields an RT / decompression contribution and a near constant saturation velocity. The spike velocity is significantly lower than that of the bubble. 
positions are extracted from a 1D CALE simulation. Spike and bubble decompression velocities are obtained by subtracting the time dependant interface velocity from the fluid velocities at the spike and bubble positions. That is,

$$
\mathrm{v}_{\mathrm{s}}^{\text {expansion }}=\mathrm{v}_{\mathrm{s}}^{\text {fluid }}-\mathrm{u}_{\mathrm{i}} \text {, }
$$

where $\mathrm{V}_{\mathrm{s}}^{\text {fluid }}$ is the velocity of the fluid at the position of the spike tip (from a 1D simulation) and $\mathrm{u}_{\mathrm{i}}$ is the interface velocity, and analogously for the bubble. An estimate of the RT contribution is then calculated as

$$
\mathrm{V}_{\mathrm{s}, \mathrm{b}}{ }^{\mathrm{RT}}=\mathrm{V}_{\mathrm{s}, \mathrm{b}}-\mathrm{V}_{\mathrm{s}, \mathrm{b}} \text { expansion }^{\text {. }} \mathrm{V}_{\mathrm{s}, \mathrm{b}}^{\text {Pure }} \mathrm{RM}
$$

where $\mathrm{v}_{\mathrm{s}, \mathrm{b}}^{\text {Pure RM }}$ are the spike and bubble velocities from the $15 \mathrm{~ns}$ (pure $\mathrm{RM}$ ) case. The time-dependant integrals of the functions $\mathrm{V}_{\mathrm{s}, \mathrm{b}}{ }^{\mathrm{RT}}$ give the decompression amplitude histories. The result of this procedure applied to the large amplitude $6 \mathrm{~ns}$ pulse case is shown in Fig. 22, which shows that $\mathrm{v}_{\mathrm{s}}^{\mathrm{RT}} \approx$ const $=0.2 \mu \mathrm{m} / \mathrm{ns}$ throughout and $\mathrm{v}_{\mathrm{b}}{ }^{\mathrm{RT}}<0$ until after $20 \mathrm{~ns}$. This suggests that RT effects are negligible except for the bubble after $22 \mathrm{~ns}$.

The same method has been used to subtract the decompression effect from the simulation of the experiment (see Fig. 23). The process is complicated by the additional compression resulting from the second small shock from $17-20 \mathrm{~ns}$, but the asymptotic result is still insightful. After removal of expansion effects, the bubble velocity reaches a peak value equal to $(\mathrm{da} / \mathrm{dt})_{\mathrm{MB}}$, and then begins to fall off, though it does so more slowly than in the pure RM simulation. The asymptotic spike velocity is down from $\approx 3 \mu \mathrm{m} / \mathrm{ns}$ to $\approx 1.5 \mu \mathrm{m} / \mathrm{ns}$. This reduction is approximately equal to that 

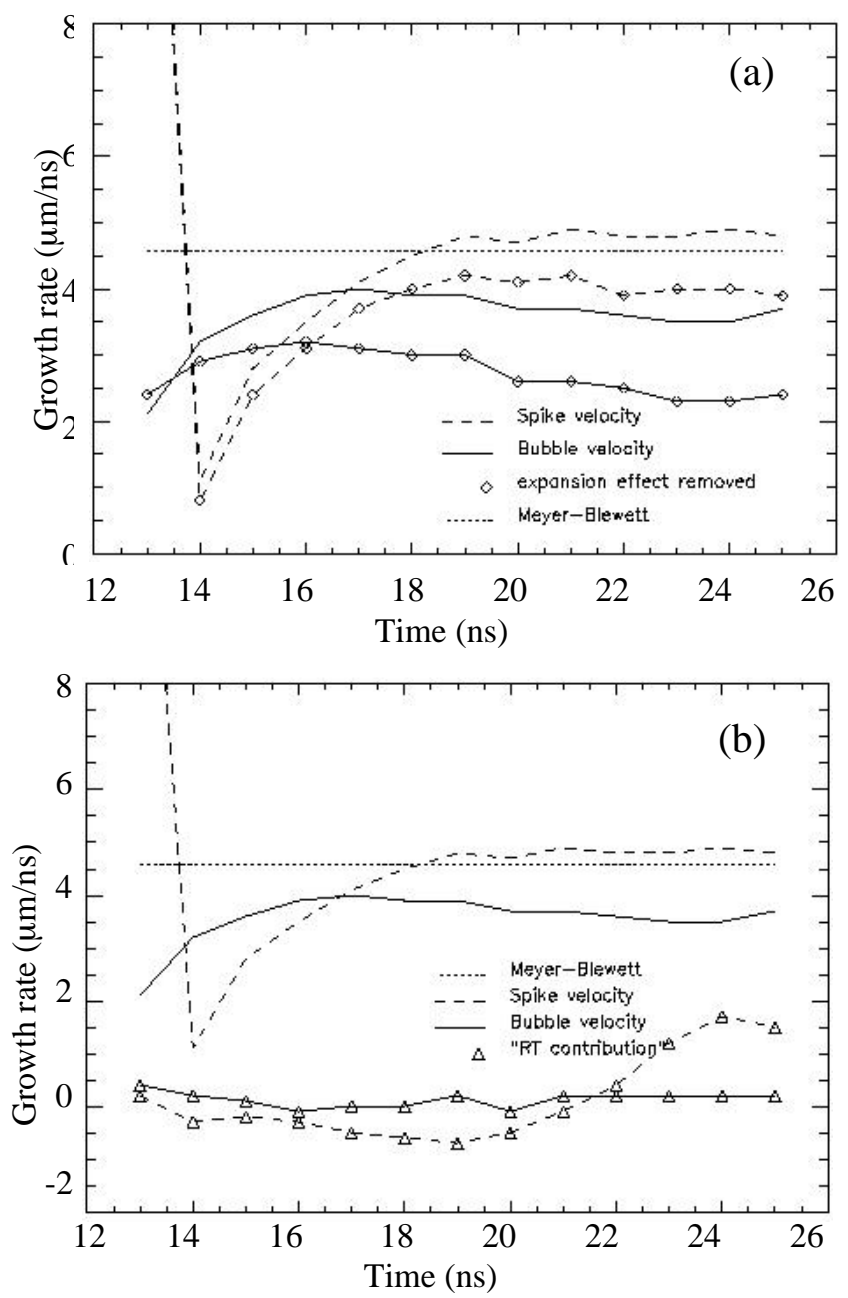

Figure 22: RT / decompression effects for large initial amplitude $(22 \mu \mathrm{m})$ driven by 6 ns constant velocity (14 $\mu \mathrm{m} / \mathrm{ns}$ ) drive: (a) Decompression-corrected spike and bubble velocities. (b) RT contribution to spike and bubble velocities obtained by subtracting decompression-corrected curves in (a) and RM-contribution from Figure 22(b) from observed velocity curves in Figure 22(e). Note that the RT growth time, with either square or real pulse, is estimated by $\tau_{\mathrm{RT}}=\left(\mathrm{k} \mathrm{g} \mathrm{A}^{-1 / 2}\right.$ as about $8 \mathrm{~ns}$ (with $\mathrm{g}=0.7$ $\mu \mathrm{m} / \mathrm{ns}^{2}$ ).

seen when going from the 6 ns square pulse to the 15 ns (pure RM) pulse: $\approx 3.5$

$\mu \mathrm{m} / \mathrm{ns}$ to $\approx 2 \mu \mathrm{m} / \mathrm{ns}$. As in the idealized case, it appears that target decompression in the experiment makes a significant contribution to the perturbation growth after 21 ns, and that RT effects become significant for the bubble after 22 ns. Combined, 


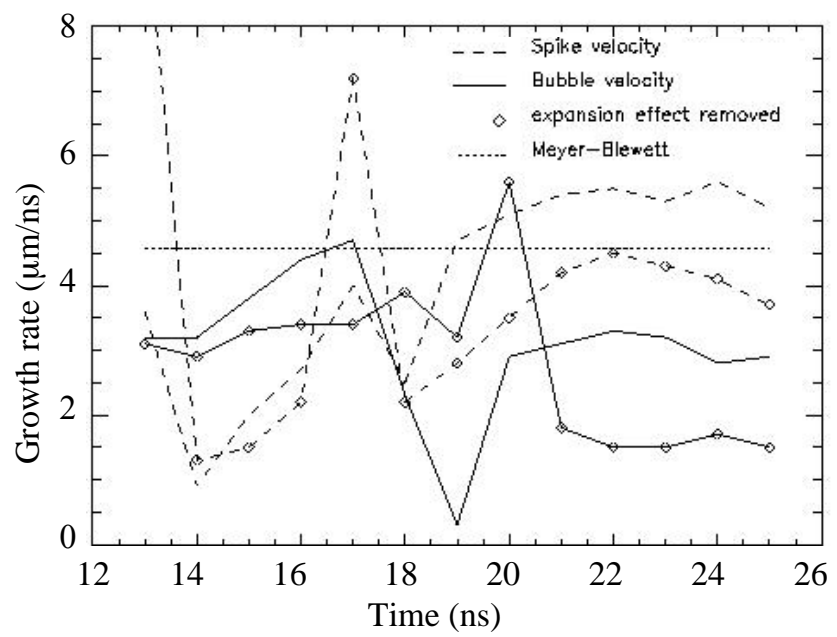

Figure 23: RT contribution in simulation with real drive (L2D). After removal of decompression effects, the bubble velocity reaches a peak value equal to $(\mathrm{da} / \mathrm{dt})_{\mathrm{MB}}$, and then begin to fall off, though it does so more slowly than in the pure RM simulation. The asymptotic spike velocity is down from $\approx 3 \mu \mathrm{m} / \mathrm{ns}$ to $\approx 1.5 \mu \mathrm{m} / \mathrm{ns}$. This reduction is approximately equal to that seen when going from the $6 \mathrm{~ns}$ square pulse to the $15 \mathrm{~ns}$ (pure RM) pulse: $\approx 3.5 \mu \mathrm{m} / \mathrm{ns}$ to $\approx 2 \mu \mathrm{m} / \mathrm{ns}$.

decompression and the RT instability account for about $10 \%$ of the growth at $20 \mathrm{~ns}$, and $20-30 \%$ at $25 \mathrm{~ns}$.

\section{$\underline{\text { V. Compressibility effects }}$}

The $\mathrm{M} \approx 10$ shocks in these experiments and simulations significantly compress the target materials, and compressibility effects are present long into the experiment. This situation can be contrasted with most non-high energy density experiments, including those conducted at shock tube facilities, where post-shock pressures are far less than $1 \mathrm{Mbar}$. In low Mach number $(\mathrm{M} \leq 3) \mathrm{RM}$ experiments, the peak instability growth rate is reached on a time scale short compared to the growth time, and the transmitted shock speed relative to the interface is much greater than perturbation growth rate. In simulations of the OMEGA experiments, the growth rate 
is initially zero, and the time to reach peak growth rate is comparable to the time of the experiment. This is illustrated by (for example) Fig. 10, which shows the spike and bubble velocities as a function of time. Models of the instability that are based on incompressible flow after shock refraction and therefore give a nonzero growth rate at zero time are not likely to accurately predict the linear growth rate in such cases. This is demonstrated in Fig. 24, which shows several such models compared to the pure RM CALE simulations for both small and large initial amplitudes. The models included in Fig. 24(a) are the Meyer-Blewett impulsive model, ${ }^{3}$ the phenomenological Sadot model, ${ }^{18}$ which combines an early time linear constantvelocity stage with an asymptotic, nonlinear, 1/t decay, the arbitrary-Atwood-number buyonacy-drag model of Oron et al.,${ }^{12}$ and Robey's image vortex model. ${ }^{15}$ The discrepancy is greatest for the large amplitude case for reasons that will be discussed in the next section. In Fig. 24(b), a comparison is made with the asymptotic (latetime) buoyancy-drag model prediction. The model predicts asymptotic spike and bubble velocities of the form $\mathrm{v}_{\text {asy }}{ }^{\mathrm{s} / \mathrm{b}}=\mathrm{C}_{\mathrm{s} / \mathrm{b}}(\mathrm{A}) / \pi \lambda / \mathrm{t}$. The value of the constant $\mathrm{C}_{\mathrm{b}}(\mathrm{A})$ agrees with the potential flow model of Hecht $e t a l .{ }^{8}$ at $\mathrm{A}=1$ and with the vortex model of Rikanati et al. ${ }^{10}$ at $\mathrm{A}=0$. It is possible to bring the model into agreement with the simulation asymptotic bubble velocity by introducing a time shift to compensate for early time compressibility effects, as was done in Figure 24(b). However, such an ad hoc model, which basically requires that one already know the answer prior to its application, would be far from compelling. Consequently, only compressible-flow models can satisfactorily predict RM growth under the conditions present in the OMEGA experiments and relevant simulations. 

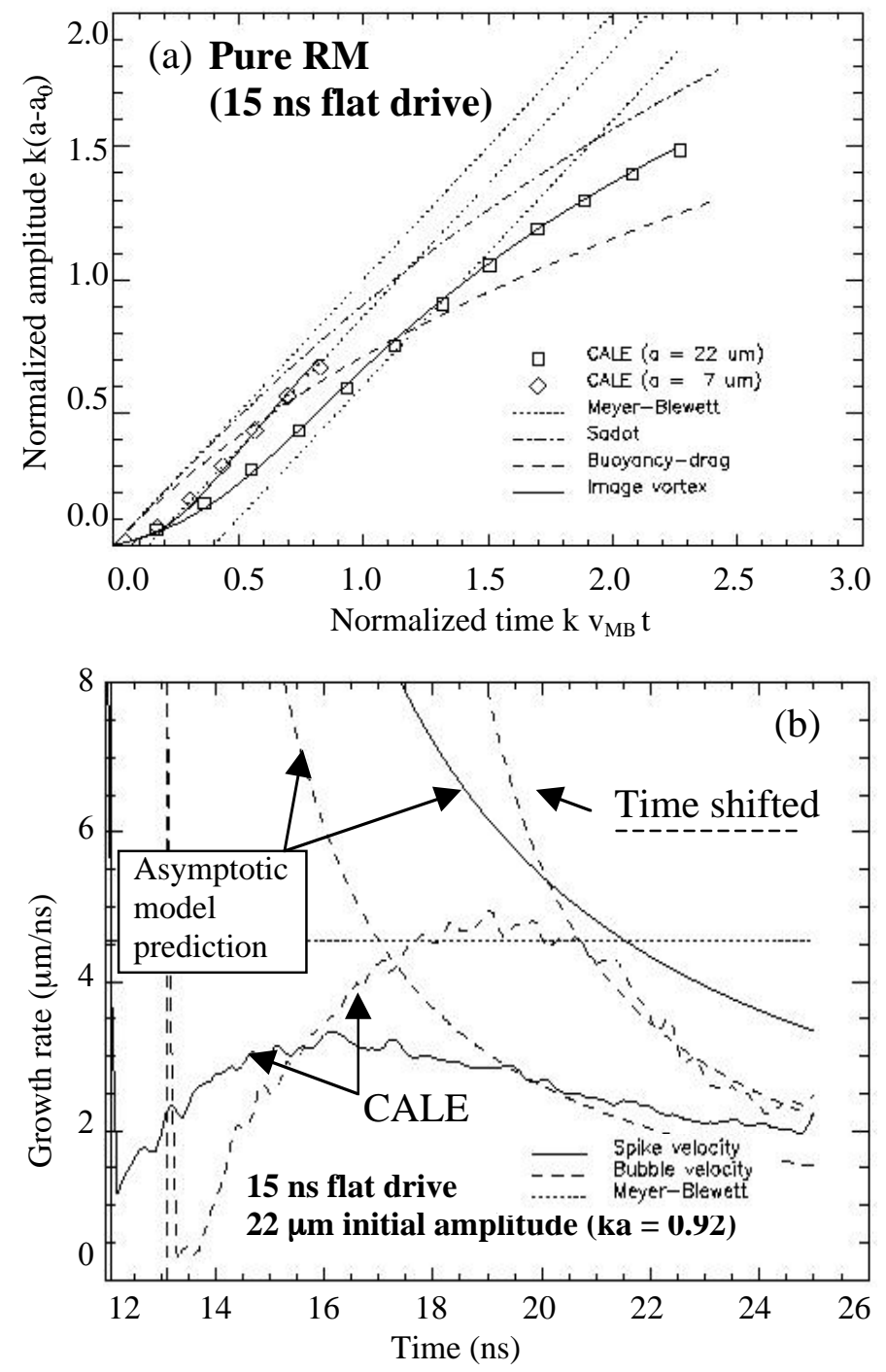

Figure 24: Model predictions and simulations for large and small initial amplitude (a $=22 \mu \mathrm{m}->\mathrm{ka}=0.92$ and $\mathrm{a}=7 \mu \mathrm{m}->\mathrm{ka}=0.29)$ with pure RM drive ( $15 \mathrm{~ns}$ flat): (a) Even when the perturbation remains in the linear regime, models based on incompressible flow fail when compressibility effects remain important long after shock refraction. The small amplitude case deviates from the Meyer-Blewett velocity only in the first couple of nanoseconds. The $22 \mu \mathrm{m}$ initial amplitude case is nowhere in agreement with the Meyer-Blewett prediction. (b) Spike and bubble velocities for large amplitude case. Potential flow and vortex models predict an asymptotic bubble velocity $\mathrm{v}_{\text {asy }}=\mathrm{C} / \pi \lambda / \mathrm{t}$. The buoyancy-drag model of Oron $e$ t $a l^{12}$ gives $\mathrm{C}=(1 / 3+((1-$ A)/(1+A))/6 ). A time shift introduced into the models to compensate for early time compressibility effects brings it into agreement with the simulation asymptotic bubble velocity. 


\section{Shock proximity}

In high-Mach-number RM systems, it is possible for the Meyer-Blewettpredicted velocity to exceed the speed of the transmitted shock relative to the unperturbed interface. If the perturbation were to nevertheless grow according to the incompressible-theory prediction, then the spikes would necessarily penetrate ahead of the zero-order shock position (actually, each spike would a drive a faster shock into the un-shocked upstream material, effectively driving the preexisting perturbation in the shock front to larger amplitude). Such behavior has not been observed. Instead, the perturbation growth rate (or at least that of the spike) appears instead to be inhibited, while the shock front is perhaps slightly distorted. While this behavior should be present in the linear and nonlinear ${ }^{17}$ compressible theories, it has in the past been incorporated into incompressible flow models in a somewhat ad hoc fashion - by simply limiting the perturbation growth rate to the relative shockinterface velocity. ${ }^{21,25}$ More recently, Robey et al.${ }^{15}$ have proposed a compressible extension to the incompressible vortex model of the RM instability that links growth reduction due to shock proximity to the boundary condition at the transmitted shock. They assume a planar shock and invoke a line of "image vortices" to enforce the appropriate boundary condition. These image vortices are located on an "image interface" that moves at twice the shock speed in the interface frame. This ensures that the parallel (to the shock-propagation direction) flow velocity just behind the shock front remains zero. 

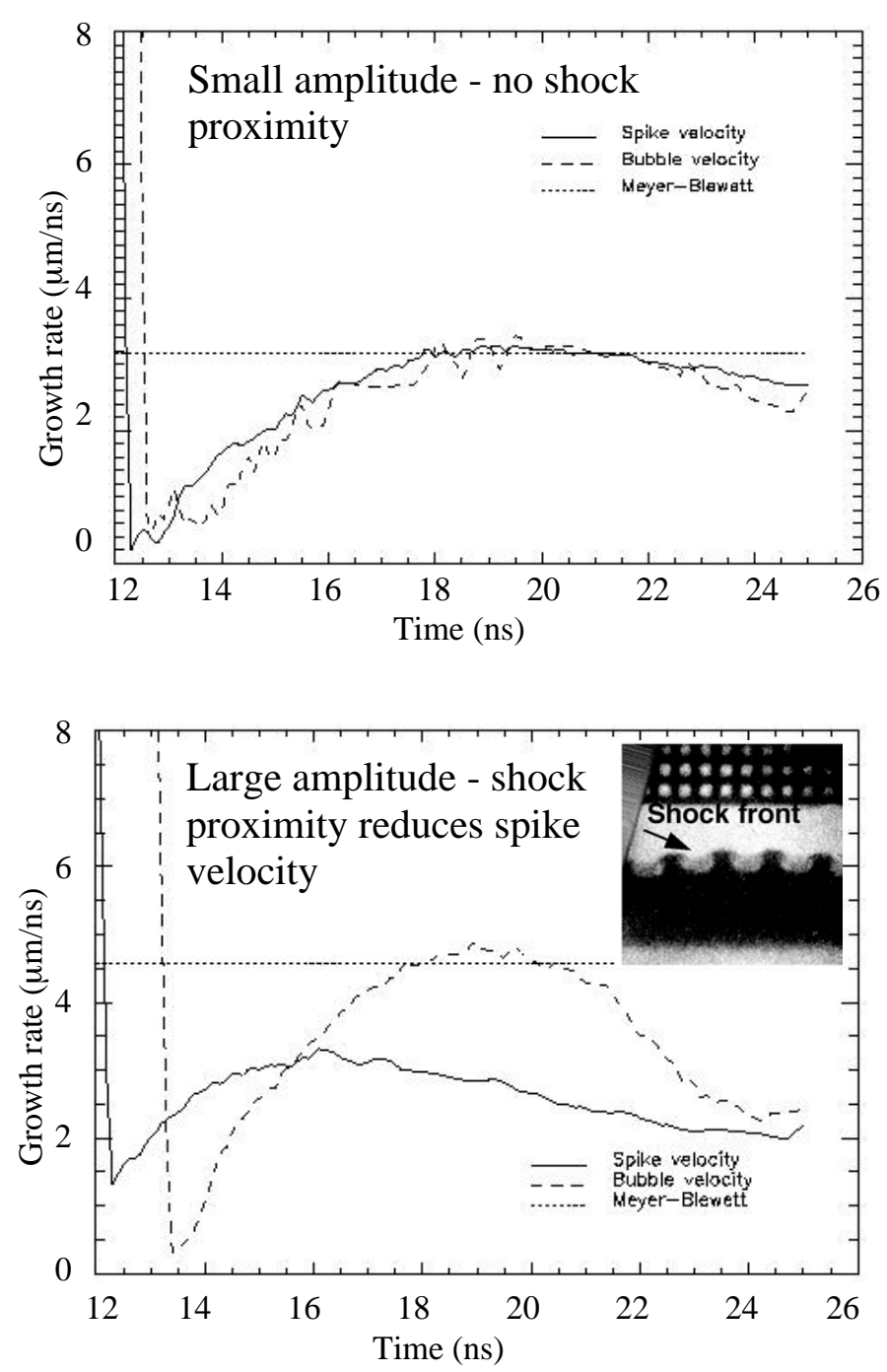

Figure 25: Shock proximity (15 ns "pure RM" flat drive, $v_{t}-u_{i}=5.4 \mu \mathrm{m} / \mathrm{ns}$ ): When the model-predicted RM growth rate is greater than the speed of the transmitted shock relative to the interface, the spikes grow up to the position of the shock and their growth is inhibited.

The pure RM CALE simulations described in Section VIII provide a striking demonstration of spike velocity reduction due to shock proximity. Unlike the experiments, the simulations allow for the extraction of time-dependant separate spike and bubble velocities. Fig. 25 shows the result for the small initial amplitude case, in which the ratio of the speed of the transmitted shock relative to the interface 
to the Meyer-Blewett velocity is 3.1. The transmitted shock quickly pulls away from the evolving perturbation without inhibiting its growth. In the large initial amplitude case, the analogous ratio is only 1.1 , so that the transmitted shock remains close to the interface. It is clear from Fig. 25 that, while the maximum bubble velocity again agrees with the Meyer-Blewett prediction (despite the somewhat large amplitude), the peak spike velocity is reduced to only $0.7(\mathrm{da} / \mathrm{dt})_{\mathrm{MB}}$.

Despite the presence of RT effects, the image vortex model predictions are in good agreement with the data from both small and large initial amplitude experiments [see Fig. 26(a)]. The agreement with the averaged amplitude in the pure RM CALE simulations is better still [see Fig. 24(a)]. Figure 26(b) also shows the image vortex model predictions of the separate spike and bubble compared to that observed in the pure RM simulation. The simulation results show that, due to the finite initial amplitude, spike growth begins 1.3 ns before bubble growth. When this is taken into account in initializing the model, it correctly predicts that, though the proximate shock ultimately affects the spike more strongly than it does the bubble, the spike is larger than the bubble for the first several ns. However, the predicted spike and bubble velocity histories are not in good qualitative agreement with the simulation. The discrepancy likely results from some combination of three model assumptions that are not satisfied in the simulation: an Atwood number of zero, a planar transmitted shock, and incompressible flow after shock refraction. In order to bring the model into agreement with the simulation, we must introduce three parameters that can be motivated, but which have not been justified. Two of the parameters are 
time increments that effectively shift the zero-time for the spike and bubble (back 1.4

ns
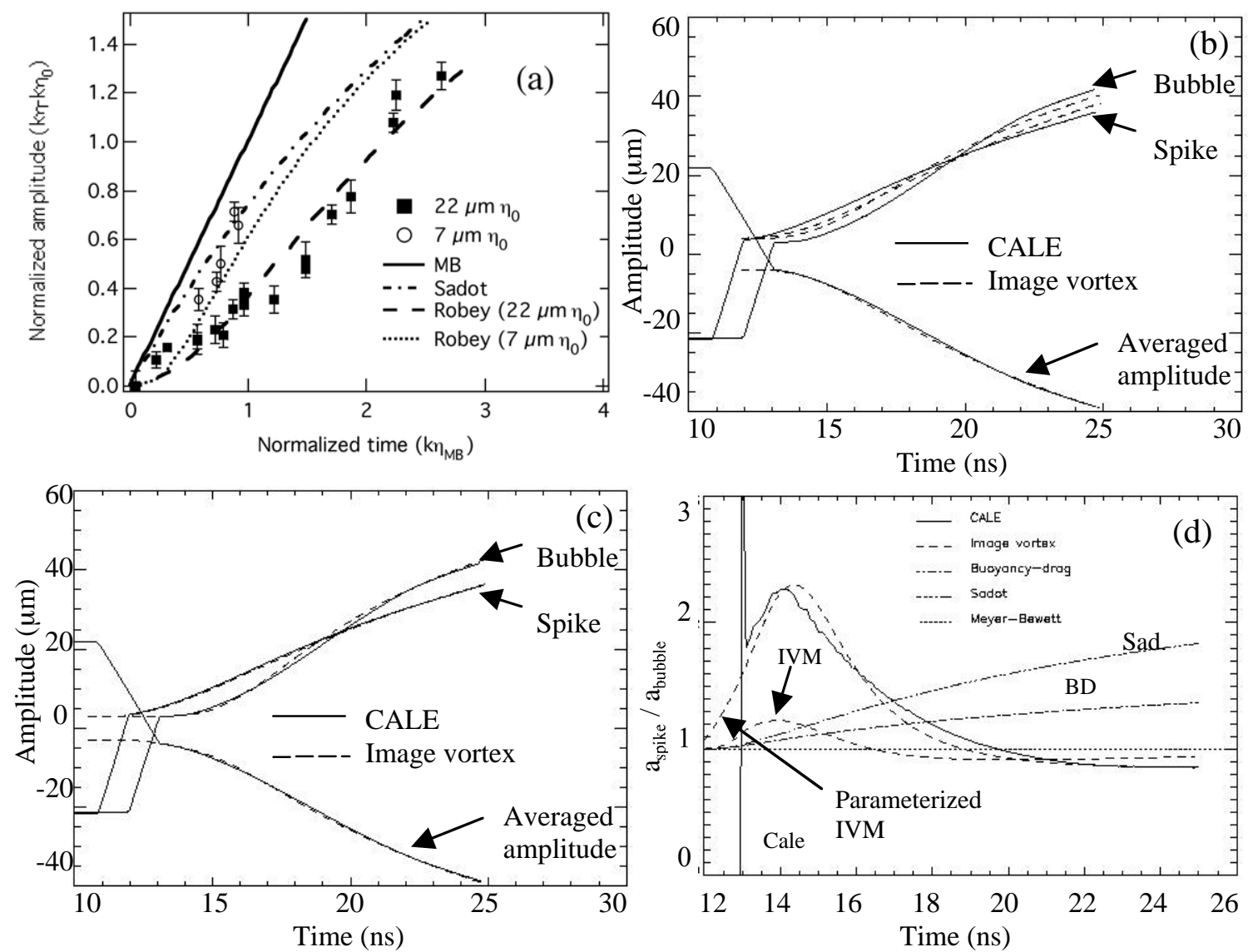

Figure 26: Image vortex model (IVM) prediction: (a) Experiment [from Glendinning et al., Phys. Plasmas 10(5). 1931 (2003)] (b) pure RM case ( c ) pure RM case with additional model parameters. In (b) and ( c ), the averaged amplitude is plotted with negative amplitude to make the plots easier to view. (d) Spike/bubble amplitude ratios.

for the spike and forward $0.6 \mathrm{~ns}$ for the bubble), and the third is a multiplicative factor of $2 / \pi$ applied to the circulation that drives the spike growth. The time shifts might account for the non-planarity of the transmitted shock, which effectively concentrates image vorticity into the (real) bubble region. Foam compression in the region 
between the spike and the transmitted shock would result in enhanced drag on the spike, and this is the motivation for the multiplicative factor. The result is shown in Fig. 26(c).

Only the image vortex model captures the observed spike-bubble asymmetry behavior [see Fig. 26(d)]. The spike amplitude is greater than that of the bubble at early times - primarily because its growth begins $1 \mathrm{~ns}$ earlier. But the spike growth is inhibited, and its amplitude eventually surpassed by the bubble. Linear theories predict symmetric spike-bubble growth. Incompressible shock proximity models ${ }^{21,25}$ incorporate growth reduction but no asymmetry. The Sadot and buoyancy-drag models, which ignore the transmitted shock, predict that spikes grow faster than bubbles.

The observed asymmetry between the spike and bubble velocities also points to a limitation of the compressible linear theories and the compressible nonlinear theories such as that of Zhang and Sohn. ${ }^{17}$ These theories are based on expansions of the fluid equations in the small parameter $a / \lambda$ : to first order in the linear theories and higher order in nonlinear theories. At sufficiently early time, the nonlinear theory will of course agree with its linear counterpart. While the boundary condition of the transmitted shock is included in the theory, it must affect the spike and bubble in a symmetrical fashion as long as the condition $a / \lambda<<1$ is satisfied (ie in the linear regime). As a/ $\lambda$ "becomes finite" and continues to grow, the equality of spike and bubble velocity will be broken. As previously noted, the Zhang and Sohn theory actually predicts that the spike velocity exceeds that of the bubble at all times, in contrast to what we have observed. 
In the context of the image vortex model, the requirement that $\mathrm{a} / \boldsymbol{\lambda}$ is an infinitesimal means that the spike and bubble are equidistant from the image vortex line. Consequently, the spike and bubble growth are retarded by the same amount, and so their amplitudes remain equal to each other. But since the spike is actually closer to the image vortex line than is the unperturbed interface while the bubble is further away, the linear theory (or the nonlinear theory in the linear regime) will overstate the bubble growth reduction and understate the spike growth reduction. The net effect on the averaged amplitude might be small, so that the averaged amplitude is relatively well predicted by the theory, but the separate spike and bubble velocities will not be well predicted as long as the transmitted shock remains close to the interface.

The proximity of the transmitted shock means the introduction of another length scale into the problem - namely the distance $\mathrm{s}=\mathrm{v}_{\text {rel }} \mathrm{t}$ between the transmitted shock and the unperturbed interface (where $v_{\text {rel }}$ is the speed of the transmitted shock relative to the interface and $t$ is the elapsed time since shock refraction). Since a perturbation theory cannot be assumed to correctly describe dynamics on scales smaller than the expansion parameter, the linear theories are not strictly valid when s / $\lambda<\mathrm{a} / \lambda$ or $\mathrm{s} / \mathrm{a}<1$. That is, the perturbation theories are only necessarily valid for $\mathrm{t}$ $>\mathrm{a}(\mathrm{t}) / \mathrm{v}_{\text {rel }}$. A necessary but not sufficient condition is that $\mathrm{t}>\mathrm{a}^{*} / \mathrm{v}_{\text {rel }} \equiv \tau_{\text {prox }}$, where $\tau_{\text {prox }}$ is called the proximity time. In those cases where $v_{\text {rel }}>>\max \{\mathrm{da}(\mathrm{t}) / \mathrm{dt}\} \leq$ $(\mathrm{da}(\mathrm{t}) / \mathrm{dt})_{\mathrm{MB}}$, this condition is satisfied very quickly. In other words, if the proximity time is short compared to both the perturbation growth time and the experiment duration (and the expansion parameter remains less than unity), then the spike and 
bubble evolution might be well described by perturbation theory. Otherwise, the asymmetric effect of the transmitted shock on the spike and bubble is significant, and one must resort to a non-perturbative theory with some provision for compressibility effects, such as the image vortex model.

Shock proximity reduction has been considered as a possibly significant effect in previous experiments. ${ }^{21,22,26}$ However, the analyses of these experiments have been complicated by the fact that the reduction was observed at a time when the instability had already developed well into the nonlinear stage. Consequently, it has proven difficult to convincingly separate shock proximity reduction from large amplitude effects, which also result in reduction of the growth rate below the predictions of linear theories. In our simulations, however, the observed strength and nature of the asymmetry between spike and bubble velocities, together with the relatively low degree of nonlinearity (discussed below), strongly suggest shock proximity reduction rather than nonlinear effects.

\section{Large amplitude effects}

The wavenumber-weighted amplitudes (ka) for the large and small initial amplitude RM simulations are plotted together in dimensionless time in Fig. 24(a). Predictions based on linear analysis are definitely suspect by the time ka has reached a value of unity. This does not occur in the small initial amplitude case, and the Meyer-Blewett velocity prediction agrees well with the data except at very early times during establishment of the nearly constant growth rate (This early time discrepancy can in fact also be attributed to the shock proximity effect). 
In the large amplitude case, the perturbation does grow into the nonlinear phase, though not nearly to the extent seen in earlier similar experiments. ${ }^{19,20,22}$ Nowhere does the Meyer-Blewett prediction agree well with the data, even during the linear phase. The peak averaged growth rate is $0.85(\mathrm{da} / \mathrm{dt})_{\mathrm{MB}}$. The perturbative expansion of Velikovich and Dimonte ${ }^{16}$ predicts (to 8 th order in ka) a reduction factor of 0.84 . The Oron $e t$ al. model $1^{12}$ predicts a slightly larger value of about 0.9. At first glance, it appears that large-amplitude effects can in fact explain the observed growth rate reduction. But again, consideration of separate spike and bubble velocities sheds additional light. The expansion referred to above gives (also to $8^{\text {th }}$ order in ka) separate reduction factors of 0.86 for the bubble and 0.82 for the spike. That is, the higher-order model treats the spike and bubble nearly symmetrically. As previously mentioned, however, we observe reduction factors of 1.0 for the bubble and 0.7 for the spike. Furthermore, the reduction on perturbation growth rate is observed from very early times, when the instability is clearly still in the linear phase. Taken together, these two facts strongly suggest that the dominant growth rate reduction mechanism is shock proximity rather than large amplitude effects.

\section{Conclusion}

We have performed CALE simulations of high Mach number direct drive RM experiments with single-mode perturbations. The experiments are extremely well characterized and diagnosed, thereby placing a high level of constraint on the simulation results. In order to accurately simulate the experiments, the gap must be 
included, and a well-constrained time dependent drive must be employed to correctly capture the series of weak shock and rarefaction waves that follow the main shock front. We have shown that a velocity drive extracted from a 2D LASNEX simulation does well, but that its 1D analogue is unsatisfactory. The instability evolution is sensitive to the material EOS, and the EOP tables are preferable to either perfect gas or LEOS. The long-term bubble-shock proximity observed in the experiments suggests that the foam may more compressible than predicted by the EOP tables, and is certainly more compressible than predicted by the LEOS tables. With a rectangular mesh, convergence is reached in an ALE calculation with a transverse resolution of 120 ppw.

When these conditions are met, the simulations accurately model the experiments down to the level of the experimental resolution. Where there are discrepancies, such as in the post-shock amplitude and bubble-shock proximity, the difference between experiment and simulation is relatively small and can be attributed to imperfect reproduction of small details in the velocity drive and perhaps the EOS. The amplitude history is in excellent agreement with the data (except for its overprediction of $a^{*}$ ) throughout the experiment.

The ability to accurately model the experiment allows for analysis of the important physics issues beyond what can be done based on experimental data alone. The ability in the simulations to follow the spike and bubble evolution separately allows us to quantify the contribution of target decompression and the RT instability to the perturbation growth. We have shown that together they contribute about $10 \%$ of the growth at $20 \mathrm{~ns}$, and $20-30 \%$ by the end of the experiment at $25 \mathrm{~ns}$. 
Considering the experimental resolution, these effects are significant after about 21

ns.

Shock proximity reduction of the perturbation growth rate is strongly suggested by the experiments. The ability in the simulations to eliminate RT and decompression effects has provided the opportunity to clearly demonstrate the shock proximity effect. In the small amplitude case, the transmitted shock quickly moves far ahead of the interface, and the spike and bubble growth is nearly symmetric. In the large initial amplitude case, where the shock remains close to the interface, the final bubble amplitude is $20 \%$ greater than that of the spike. Based on its amplitude, the perturbation is only weakly nonlinear even at late times. The conclusion that shock proximity is responsible for the observed growth suppression is strengthened by the fact that existing nonlinear models predict nearly symmetric reduction of spikes and bubbles in this regime.

The same ability has facilitated more relevant comparison with models and, consequently, demonstration of the inapplicability of incompressible flow models (or any model that gives a non-zero growth rate at $\mathrm{t}=0$ ) for high Mach number RM experiments. The compressible linear theory has also been shown to be deficient in this case, while Robey's image vortex model qualitatively captures the evolution and may be more generally applicable. 


\section{References}

\section{Chapter 1}

${ }^{1}$ S. W. Falk and W. D. Arnett, Astrophys. J. Letters, 180, L65 (1973).

${ }^{2}$ R. Chevalier, Ap. J. 207, 872 (1976).

${ }^{3}$ J. Tueller, S. Barthelmy, N. Gehrels. B. J. Teegarden, M. Leventhal, and C. J. MacCallum, Astrophys. J. 351, L41 (1990).

${ }^{4}$ L. Wang, D. Baade, P. Höflich, A. Khokhlov, J. C. Wheeler, et al., Astrophys. J. 591, 1110 (2003).

${ }^{5}$ J. P. Hughes, C. E. Rakowski, D. N. Burrows, and P. O. Slane, Astrophys. J. 528, L109 (2000).

${ }^{6}$ A. M. Khokhlov, P. A. Höflich, E. S. Oran, J. C. Wheeler, L. Wang, and A. Yu. Chtchelkanova, Astrophys. J. 524, L107 (1999).

${ }^{7}$ W. D. Arnett, J. N. Bachall, R. P. Kirshner, and S. E. Woosley, Ann. Rev. Astron. Astrophys. 27, 629 (1989).

${ }^{8}$ W. Hillebrandt and P. Hoflich, Rep. Prog. Phys. 52, 1421 (1989).

${ }^{9}$ D. Arnett, B. Fryxell, E. Muller, Astrophys. J. 341, L63 (1989); E. Muller, B. Fryxell, D. Arnett, Astron. Astrophys. 251, 505 (1991)

${ }^{10}$ B. A. Remington, J. Kane, R. P. Drake, et al., Phys. Plasmas 4, 1994 (1997).

${ }^{11}$ J. W. S. Rayleigh, Scientific Papers (University press, Cambridge, 1899).

${ }^{12}$ G. I. Taylor, Proc. R. Soc. London, Ser. A 201, 192 (1950).

${ }^{13}$ R. D. Richtmyer, Commun. Pure Appl. Math. 13, 297 (1960). 
${ }^{14}$ E. E. Meshkov, Izv. AN SSSR Mekhanika Zhidkosti I Gaza 4(5), 151 (1969).

${ }^{15}$ John D. Lindl, Inertial Confinement Fusion: the quest for ignition and high-energy

gain, (Springer-Verlag, New York, 1998).

${ }^{16}$ J. L. Milovich, P. Amendt, M. Marinak, and H. Robey, Phys. Plasmas 11(4), (2004).

${ }^{17}$ J. Hecht, U. Alon, and D. Shvarts, Phys. Fluids 6, 4019 (1994).

${ }^{18}$ U. Alon, J. Hecht, D. Ofer, and D. Shvarts, Phys. Rev. Lett. 74, 534 (1995).

${ }^{19}$ J. M. Soures, R. L. McCrory, C. P. Verdon, et al., Phys. Plasmas 5, 2108 (1996).

${ }^{20}$ K. S. Budil, B. A. Remington, T. A. Peyser, K. O. Mikaelian, P. L. Miller, et al., Phys. Rev. Lett., 76, 4536 (1996).

${ }^{21}$ J. Kane, D. Arnett, B. A. Remington, S. G. Glendinning, J. Castor, et al., Astrophys. J., 478, L75 (1997).

${ }^{22}$ J. Kane, D. Arnett, B. A. Remington, S. G. Glendinning, R. Wallace, et al., in Second Oak Ridge Symposium on Atomic and Nuclear Astrophysics, Oak Ridge, Tenessee, (1998).

${ }^{23}$ J. Kane, D. Arnett, B. A. Remington, S. G. Glendinning, G. Bazan, R. P. Drake, B. A. Fryxell, R. Teyssier, and K. Moore, Phys. Plasmas, 6(5), 2065 (1999).

${ }^{24}$ J. O. Kane, H. F. Robey, B. A. Remington, R. P. Drake, J. Knauer, et al., Phys. Rev. E, 63, 055401R (2001).

${ }^{25}$ H. F. Robey, J. O. Kane, B. A. Remington, R. P. Drake, O. A. Hurricane, et al., Phys. Plasmas, 8, 2446 (2001).

${ }^{26}$ R. P. Drake, H. F. Robey, O. A. Hurricane, Y. Zhang, B. A. Remington, et al., Astrophys. J., 564, 896 (2002).

${ }^{27}$ H. F. Robey, Y. Zhou, A. C. Buckingham, P. Keiter, B. A. Remington, et al., Phys. 
Plasmas, 10, 614 (2003).

${ }^{28}$ A. R. Miles, D. G. Braun, M. J. Edwards, H. F. Robey, R. P. Drake, et al., "Numerical Simulation of Supernova-Relevant Laser-driven Hydro Experiments on OMEGA," to appear in Phys. Plasmas; Chapter 2, this dissertation.

${ }^{29}$ J. Glimm, D. H. Sharp, Phys. Rev. Lett. 64(18), 2137 (1990).

${ }^{30}$ U. Alon, J. Hecht, D. Mulamel, and D. Shvarts, Phys. Rev. Lett. 72(18), 2867 (1994).

${ }^{31}$ D. L. Youngs, Physica D 12, 32 (1984).

${ }^{32}$ K. I. Sharp, Physica D, 12, 3 (1984).

${ }^{33}$ J. Glimm and X. L. Li, Phys. Fluids, 31(8), 2077 (1988).

${ }^{34}$ G. Dimonte and M. Schneider, Phys. Fluids 12(2), 304 (2000).

${ }^{35}$ D. L. Youngs, Laser and Part. Beams 12(4), 725 (1994).

${ }^{36}$ K. Kifonidis, T. Plewa, H.-Th. Janka, and E. Muller, Astron. Astrophys., 408, 621 (2003).

${ }^{37}$ R. T. Barton, Numerical Astrophysics (Jones and Bartlett, Boston, 1985).

${ }^{38}$ L. H. Howell and J.A. Greenough, J. Comp. Phys. 184, 53 (2003).

${ }^{39}$ J. Bell, M. Berger, J. Saltzman, and M. Welcome, SIAM J. Sci. Comput. 15, 127 (1994).

${ }^{40}$ C. A. Meakin and W. D. Arnett, in preparation.

${ }^{41}$ J. F. Hansen, in preparation.

\section{Chapter 2}

${ }^{1}$ J. W. S. Rayleigh, Scientific Papers (University press, Cambridge, 1899). 
${ }^{2}$ G. I. Taylor, Proc. R. Soc. London, Ser. A 201, 192 (1950).

${ }^{3}$ S. Chandrasekhar, Hydrodynamic and hydromagnetic stability, (Dover Publications, New York, 1981).

${ }^{4}$ John D. Lindl, Inertial Confinement Fusion: the quest for ignition and high-energy gain, (Springer-Verlag, New York, 1998).

${ }^{5}$ Dov Shvarts, Oren Sadot, Dan Oron, Avi Rikanati, and Uri Alon G. BenDor, in Handbook of Shock Waves, edited by Gabi Ben-Dor, Ozer Igra, and Tov Elperin (Academic Press, London, 2001), Vol. 2, Chap. 14, pp. 489-543.

${ }^{6}$ H. J. Kull, Phys. Rep. 206, 197 (1991).

${ }^{7}$ M. N. Rosenbluth and C.L. Longmire, Ann. Physics, 26, 2227 (1957).

${ }^{8}$ R. Chevalier, Ap. J. 207, 872 (1976).

${ }^{9}$ Ya. B. Zel'dovich and Yu. P. Raizer, Physics of Shock Waves and High-Temperature Hydrodynamic Phenomema, (Dover Publications, New York 2002).

${ }^{10}$ National Research Council of the National Academies, Frontiers in High Energy

Density Physics, (The National Academies Press, Washington, DC 2002).

${ }^{11}$ W. D. Arnett, J. N. Bachall, R. P. Kirshner, and S. E. Woosley, Ann. Rev. Astron. Astrophys. 27, 629 (1989).

${ }^{12}$ W. Hillebrandt and P. Hoflich, Rep. Prog. Phys. 52, 1421 (1989).

${ }^{13}$ D. Ryutov, R. P. Drake, J. Kane, E. Liang, B. A. Remington, and W. M. WoodVasey, Astrophys. J. 518, 821 (1999).

${ }^{14}$ D. Arnett, B. Fryxell, E. Muller, Astrophys. J. 341, L63 (1989); E. Muller, B.

Fryxell, D. Arnett, Astron. Astrophys. 251, 505 (1991)

${ }^{15}$ B. A. Remington, J. Kane, R. P. Drake, et al., Phys. Plasmas 4, 1994 (1997). 
${ }^{16}$ J. Hecht, U. Alon, and D. Shvarts, Phys. Fluids 6, 4019 (1994).

${ }^{17}$ U. Alon, J. Hecht, D. Ofer, and D. Shvarts, Phys. Rev. Lett. 74, 534 (1995).

${ }^{18}$ J. M. Soures, R. L. McCrory, C. P. Verdon, et al., Phys. Plasmas 5, 2108 (1996).

${ }^{19}$ J. Kane, D. Arnett, B. A. Remington, S. G. Glendinning, J. Castor, R. Wallace, A. Rubenchik, and B. A. Fryxell, Astrophys. J. Lett. 478, L75 (1997).

${ }^{20}$ B. A. Remington, R. P. Drake, H. Takabe, and D. Arnett, Phys. Plasmas 7(5), 1641 (2000).

${ }^{21}$ R. P. Drake, J. J. Carroll, K. Eastbrook, S. G. Glendinning, B. A. Remington, and R. McCray, Astrophys. J. Lett. 500, L157 (1998).

${ }^{22}$ P. E. Dimotakis, J. Fluid Mech. 409, 69 (2000).

${ }^{23}$ H. F. Robey, Ye Zhou, A. C. Buckingham, P. Keiter, B. A. Remington, and R. P.

Drake, Phys. Plasmas 10, 614 (2003).

${ }^{24}$ R. T. Barton, Numerical Astrophysics (Jones and Bartlett, Boston, 1985).

${ }^{25}$ R. D. Richtmyer, Commun. Pure Appl. Math. 13, 297 (1960).

${ }^{26}$ E. E. Meshkov, Izv. AN SSSR Mekhanika Zhidkosti I Gaza 4(5), 151 (1969).

${ }^{27}$ S. G. Glendinning, J. Bolstad, D. G. Braun, et al., Phys. Plasmas 10(5). 1931 (2003).

${ }^{28}$ R. M. More, K. H. Warren, D. A. Young, and G. B. Zimmerman, Phys. Fluids 31(10), 3059 (1988).

${ }^{29}$ S. V. Weber, B. A. Remington, S. W. Haan, B. G. Wilson, and J. K. Nash, Phys. Plasmas 1(11), 3652 (1994).

${ }^{30}$ G. B. Zimmerman and W. L. Kruer, Comments on Plasma Physics and Controlled Fusion 2, 51 (1975). 
${ }^{31}$ V. N. Goncharov, P. McKenty, S. Shupsky, R. Betti, R. L. McCrory, and C.

Cherfils-Clerouin, Phys. Plasmas 7(12), 5118 (2000).

${ }^{32}$ D. Oron, L. Arazi, D. Kartoon, A. Rikanati, U. Alon, and D. Shvarts, Phys. Plasmas 8(6), 2883 (2001).

${ }^{33}$ J. C. V. Hanson, P. A. Rosen, T. J. Goldsack, K. Oades, P. Fieldhouse, N.

Cowperthwaite, D. L. Youngs, N. Mawhinney, and A. J. Baxter, Laser Part. Beams 8, 51 (1990).

${ }^{34}$ G. Dimonte, Phys. Plasmas 7(6), 2255 (2000).

${ }^{35}$ K. A. Meyer and P. J. Blewett, Phys. Fluids 15(5), 753 (1972).

\section{Chapter 3}

${ }^{1}$ W. D. Arnett et al., Ann. Rev. Astron. Astrophys. 27, 629 (1989).

${ }^{2}$ W. Hillebrandt and P. Hoflich, Rep. Prog. Phys. 52, 1421 (1989).

${ }^{3}$ R. Chevalier, Ap. J. 207, 872 (1976).

${ }^{4}$ R. D. Richtmyer, Commun. Pure Appl. Math. 13, 297 (1960).

${ }^{5}$ E. E. Meshkov, Izv. AN SSSR Mekhanika Zhidkosti I Gaza 4(5), 151 (1969).

${ }^{6}$ J. W. S. Rayleigh, Scientific Papers (University press, Cambridge, 1899).

${ }^{7}$ G. I. Taylor, Proc. R. Soc. London, Ser. A 201, 192 (1950).

${ }^{8}$ Amendt et al., Phys. Plasmas 9(5), 2221 (2002).

${ }^{9}$ D. Ryutov et al., Ap. J. 518, 821 (1999).

${ }^{10}$ P. E. Dimotakis, J. Fluid Mech. 409, 69 (2000).

${ }^{11}$ H. F. Robey et al., Phys. Plasmas 10, 614 (2003).

${ }^{12}$ B. Fryxell, E. Muller, and D. Arnett, Ap. J. 367, 619 (1991). 
${ }^{13}$ J. M. Soures et al., Phys. Plasmas 3, 2108 (1996).

${ }^{14}$ R. T. Barton, Numerical Astrophysics (Jones and Bartlett, Boston, 1985).

${ }^{15}$ A. R. Miles et al., "Numerical Simulation of Supernova-Relevant Laser-driven

Hydro Experiments on OMEGA”, to appear in Phys. Plasmas; Chapter 2, this

Dissertation.

${ }^{16}$ G. B. Zimmerman and W. L. Kruer, Comments on Plasma Physics and Controlled Fusion 2, 51 (1975).

${ }^{17}$ D. Ofer, U. Alon, D. Shvarts, R. L. McCroy, and C. P. Verdon, Phys. Plasmas 3(8), 3073 (1996).

${ }^{18}$ D. Ofer, D. Shvarts, Z. Zinamon, and S. A. Orszag, Phys. Fluids B 4(11), 3549 (1992).

${ }^{19}$ S. Chandrasekhar, Hydrodynamic and hydromagnetic stability, (Dover Publications, New York, 1981).

${ }^{20}$ J. Lindl, Inertial Confinement Fusion: the quest for ignition and high-energy gain, (Springer-Verlag, New York, 1998).

${ }^{21}$ K. A. Meyer and P. J. Blewett, Phys. Fluids 15(5), 753 (1972).

${ }^{22}$ S. W. Haan, Phys. Fluids B 3(8), 2349 (1991).

${ }^{23}$ S. W. Haan, Phys. Rev. A 39(11), 5812 (1989).

${ }^{24}$ G. BenDor et al., (Academic Press, London, 2001), Vol. 2, p. 489.

${ }^{25}$ H. J. Kull, Phys. Rep. 206, 197 (1991).

${ }^{26}$ D. H. Sharp, Physica D 12, 3 (1984).

${ }^{27}$ J. Glimm and D. H. Sharp, Phys. Rev. Lett. 64(18), 2137 (1990).

${ }^{28}$ D. Shvarts, U. Alon, D. Ofer, R. L. McCroy, and C. P. Verdon, Phys. Plasmas 2(6), 
$2465(1995)$.

${ }^{29}$ K. I. Read, Physica D 12, 45 (1984).

${ }^{30}$ G. Dimonte and M. Schneider, Phys. Fluids 12(2), 304 (2000).

${ }^{31}$ D. L. Youngs, Physica D 12, 32 (1984).

${ }^{32}$ D. Oron et al., Phys. Plasmas 8(6), 2883 (2001).

${ }^{33}$ R. L. Panton, Incompressible Flow, $2^{\text {nd }}$ Edition, (John Wiley \& Sons, Inc., New York 1996).

${ }^{34}$ X. L. Li, Phys. Fluids 8(2), 336 (1996).

\section{Chapter 4}

${ }^{1}$ J. W. S. Rayleigh, Scientific Papers (University press, Cambridge, 1899).

${ }^{2}$ G. I. Taylor, Proc. R. Soc. London, Ser. A 201, 192 (1950).

${ }^{3}$ S. Chandrasekhar, Hydrodynamic and hydromagnetic stability, (Dover Publications, New York, 1981).

${ }^{4}$ John D. Lindl, Inertial Confinement Fusion: the quest for ignition and high-energy gain, (Springer-Verlag, New York, 1998).

${ }^{5}$ Dov Shvarts, Oren Sadot, Dan Oron, Avi Rikanati, and Uri Alon G. BenDor, in Handbook of Shock Waves, edited by Gabi Ben-Dor, Ozer Igra, and Tov Elperin (Academic Press, London, 2001), Vol. 2, Chap. 14, pp. 489-543.

${ }^{6}$ H. J. Kull, Phys. Rep. 206, 197 (1991).

${ }^{7}$ M. N. Rosenbluth and C.L. Longmire, Ann. Physics, 26, 2227 (1957).

${ }^{8}$ R. Chevalier, Ap. J. 207, 872 (1976).

${ }^{9}$ Ya. B. Zel'dovich and Yu. P. Raizer, Physics of Shock Waves and High-Temperature 
Hydrodynamic Phenomema, (Dover Publications, New York 2002).

${ }^{10}$ National Research Council of the National Academies, Frontiers in High Energy

Density Physics, (The National Academies Press, Washington, DC 2002).

${ }^{11}$ W. D. Arnett, J. N. Bachall, R. P. Kirshner, and S. E. Woosley, Ann. Rev. Astron. Astrophys. 27, 629 (1989).

${ }^{12}$ W. Hillebrandt and P. Hoflich, Rep. Prog. Phys. 52, 1421 (1989).

${ }^{13}$ D. Ryutov, R. P. Drake, J. Kane, E. Liang, B. A. Remington, and W. M. WoodVasey, Astrophys. J. 518, 821 (1999).

${ }^{14}$ D. Arnett, B. Fryxell, E. Muller, Astrophys. J. 341, L63 (1989); E. Muller, B.

Fryxell, D. Arnett, Astron. Astrophys. 251, 505 (1991)

${ }^{15}$ B. A. Remington, J. Kane, R. P. Drake, et al., Phys. Plasmas 4, 1994 (1997).

${ }^{16}$ K. S. Budil, B. A. Remington, T. A. Peyser, K. O. Mikaelian, P. L. Miller, et al., Phys. Rev. Lett., 76, 4536 (1996).

${ }^{17}$ J. Kane, D. Arnett, B. A. Remington, S. G. Glendinning, J. Castor, et al., Astrophys. J., 478, L75 (1997).

${ }^{18}$ J. Kane, D. Arnett, B. A. Remington, S. G. Glendinning, R. Wallace, et al., in Second Oak Ridge Symposium on Atomic and Nuclear Astrophysics, Oak Ridge, Tenessee, (1998).

${ }^{19}$ J. Kane, D. Arnett, B. A. Remington, S. G. Glendinning, G. Bazan, R. P. Drake, B.

A. Fryxell, R. Teyssier, and K. Moore, Phys. Plasmas, 6(5), 2065 (1999).

${ }^{20}$ J. O. Kane, H. F. Robey, B. A. Remington, R. P. Drake, J. Knauer, et al., Phys. Rev. E, 63, 055401R (2001).

${ }^{21}$ H. F. Robey, J. O. Kane, B. A. Remington, R. P. Drake, O. A. Hurricane, et al., 
Phys. Plasmas, 8, 2446 (2001).

${ }^{22}$ R. P. Drake, H. F. Robey, O. A. Hurricane, Y. Zhang, B. A. Remington, et al., Astrophys. J., 564, 896 (2002).

${ }^{23}$ H. F. Robey, Y. Zhou, A. C. Buckingham, P. Keiter, B. A. Remington, et al., Phys. Plasmas, 10, 614 (2003).

${ }^{24}$ A. R. Miles, D. G. Braun, M. J. Edwards, H. F. Robey, R. P. Drake, et al., "Numerical Simulation of Supernova-Relevant Laser-driven Hydro Experiments on OMEGA," to appear in Phys. Plasmas; Chapter 2, this dissertation.

${ }^{25}$ K. I. Sharp, Physica D, 12, 3 (1984).

${ }^{26}$ J. Glimm and X. L. Li, Phys. Fluids, 31(8), 2077 (1988).

${ }^{27}$ K. Kifonidis, T. Plewa, H.-Th. Janka, and E. Muller, Astron. Astrophys., 408, 621 (2003).

${ }^{28}$ C. A. Meakin and W. D. Arnett, in preparation.

${ }^{29}$ D. Oron, L. Arazi, D. Kartoon, A. Rikanati, U. Alon, and D. Shvarts, Phys. Plasmas 8(6), 2883 (2001).

${ }^{30}$ J. C. V. Hanson, P. A. Rosen, T. J. Goldsack, K. Oades, P. Fieldhouse, N. Cowperthwaite, D. L. Youngs, N. Mawhinney, and A. J. Baxter, Laser Part. Beams 8, 51 (1990).

${ }^{31}$ G. Dimonte and M. Schneider, Phys. Rev. E 54, 3740 (1996).

${ }^{32}$ D. Layzer, Ap. J. 122, 1 (1955).

${ }^{33}$ R. M. Davies and G. I. Taylor, Proc. R. Soc. London Ser. A 200, 375 (1950).

${ }^{34}$ K. I. Read, Physica D, 12, 45 (1984).

${ }^{35}$ G. Glimm, J. Grove, and X. L. Li, in Proceedings of Taormina Conference, Sicily, 
1987 (University of Mesina, Messina, Italy).

${ }^{36}$ U. Alon, D. Shvarts, and D. Mukamel, Phys. Rev. E, 48(2), 1008 (1993).

${ }^{37}$ U. Alon, J. Hecht, D. Mukamel, and D. Shvarts, Phys. Rev. Lett., 72(18), 2867 (1994).

${ }^{38}$ C. L. Gardner, J. Glimm, O. McBryan, R. Menikoff, D. H. Sharp, and Q. Zhang, Phys. Fluids 31, 447 (1998).

${ }^{39}$ J. Hecht, U. Alon, and D. Shvarts, Phys. Fluids 6, 4019 (1994).

${ }^{40}$ J. Glimm, X. L. Li, R. Menikoff, D. H. Sharp, and Q. Zhang, Phys. Fluids A 2(11), 2046 (1990).

${ }^{41}$ D. L. Youngs, Physica D, 12, 32 (1984).

${ }^{42}$ J. Glimm, D. H. Sharp, Phys. Rev. Lett. 64(18), 2137 (1990).

${ }^{43}$ G. Dimonte and M. Schneider, Phys. Fluids 12(2), 304 (2000).

${ }^{44}$ L. I. Sedov, Prikl. Mat. Mekh. 10, 241 (1946).

${ }^{45}$ L. I. Sedov, Similarity and Dimensional Methods in Mechanics, (Academic Press, New York 1959).

${ }^{46}$ G. I. Taylor, Proc. R. Soc. London A201, 159 (1950).

${ }^{47}$ L. D. Landau and E. M. Lifshitz, Fluid Mechanics, (Pergamon Press, New York 1974).

${ }^{48}$ G. I. Barenblatt, Similarity, Self-Similarity and Intermediate Asymptotics, (Consultant Bureau, New York, 1979).

${ }^{49}$ R. T. Barton, Numerical Astrophysics (Jones and Bartlett, Boston, 1985).

${ }^{50}$ R. D. Richtmyer, Commun. Pure Appl. Math. 13, 297 (1960).

${ }^{51}$ E. E. Meshkov, Izv. AN SSSR Mekhanika Zhidkosti I Gaza 4(5), 151 (1969). 
${ }^{52}$ L. H. Howell, and J. A. Greenough, J. Comp. Phys., 184, 53 (2003).

${ }^{53}$ A. R. Miles, M. J. Edwards, and J. A. Greenough, "Effect of Initial Conditions on 2D Rayleigh-Taylor Instability and Transition to Turbulence in Planar Blast-wavedriven systems", submitted to Phys. Plasmas; Chapter 5, this dissertation.

${ }^{54}$ B. Fryxell, E. Muller, and D. Arnett, Astrophys. J. 367, 619 (1991); D. Arnett, (private communication).

${ }^{55}$ J. P. Hughes, C. E. Rakowski, D. N. Burrows, and P. O. Slane, Astrophys. J. 528, L109 (2000).

${ }^{56}$ M. C. Weisskopf, S. L. O’Dell, and L. P. van Speybroeck, Proc. SPIE, 2805, 2 (1996).

${ }^{57}$ Operation Fishbowl, produced by U.S. Department of Energy (distributed by U.S. Department of Energy, National Nuclear Security Administration, Nevada Site Office, Nuclear Testing Archive, M/S 400, P.O. Box 98521 Las Vegas, NV 891938521), VHS, black and white, $28 \mathrm{~min}$.

${ }^{58}$ Nukes in Space: the Rainbow Bombs, film produced by VCE inc. (distributed by VCE inc., http://www.vce.com/atomcentral.html, released 1999), DVD, color, 52 $\min$.

\section{Chapter 5}

${ }^{1}$ J. W. S. Rayleigh, Scientific Papers (University press, Cambridge, 1899).

${ }^{2}$ G. I. Taylor, Proc. R. Soc. London, Ser. A 201, 192 (1950).

${ }^{3}$ J. Glimm, D. H. Sharp, Phys. Rev. Lett. 64(18), 2137 (1990). 
${ }^{4}$ U. Alon, J. Hecht, D. Mulamel, and D. Shvarts, Phys. Rev. Lett. 72(18), 2867 (1994).

${ }^{5}$ D. L. Youngs, Physica D 12, 32 (1984).

${ }^{6}$ K. I. Read, Physica D 12, 45 (1984).

${ }^{7}$ W. D. Arnett, J. N. Bachall, R. P. Kirshner, and S. E. Woosley, Ann. Rev. Astron. Astrophys. 27, 629 (1989).

${ }^{8}$ W. Hillebrandt and P. Hoflich, Rep. Prog. Phys. 52, 1421 (1989).

${ }^{9}$ R. D. Richtmyer, Commun. Pure Appl. Math. 13, 297 (1960).

${ }^{10}$ E. E. Meshkov, Izv. AN SSSR Mekhanika Zhidkosti I Gaza 4(5), 151 (1969).

${ }^{11}$ R. Chevalier, Ap. J. 207, 872 (1976).

${ }^{12}$ J. Kane, D. Arnett, B. A. Remington, S. G. Glendinning, G. Bazan, R. P. Drake, B. A. Fryxell, R. Teyssier, and K. Moore, Phys. Plasmas 6(5), 2065 (1999).

${ }^{13}$ A. R. Miles, D.G. Braun, M.J. Edwards, H.F. Robey, R.P. Drake, et al., "Numerical Simulation of Supernova-Relevant Laser-driven Hydro Experiments on OMEGA", to appear in Phys. Plasmas; Chapter 2, this dissertation.

${ }^{14}$ D. Arnett, B. Fryxell, E. Muller, Astrophys. J. 341, L63 (1989); E. Muller, B.

Fryxell, D. Arnett, Astron. Astrophys. 251, 505 (1991)

${ }^{15}$ B. A. Remington, J. Kane, R. P. Drake, et al., Phys. Plasmas 4, 1994 (1997).

${ }^{16}$ E. M. Campbell, Las. Part. Beams 9, 209 (1991).

${ }^{17}$ J. M. Soures, R. L. McCrory, C. P. Verdon, et al., Phys. Plasmas 5, 2108 (1996).

${ }^{18}$ K.S. Budil, B.A. Remington, T.A. Peyser, K.O. Mikaelian, P.L. Miller, et al., Phys. Rev. Lett. 76, 4536 (1996).

${ }^{19}$ J. Kane, D. Arnett, B.A. Remington, S.G. Glendinning, J. Castor, et al., Astrophys. 
J. 478, L75 (1997).

${ }^{20}$ J. Kane, D. Arnett, B.A. Remington, S.G. Glendinning, R. Wallace, et al., in Second Oak Ridge Symposium on Atomic and Nuclear Astrophysics, Oak Ridge, Tenessee, 1998).

${ }^{21}$ J. Kane, D. Arnett, B.A. Remington, S.G. Glendinning, G. Bazan, et al., Astrophys. J. Suppl. 127, 365 (2000).

${ }^{22}$ J.O. Kane, H.F. Robey, B.A. Remington, R.P. Drake, J. Knauer, et al., Phys. Rev. E 63, 055401R (2001).

${ }^{23}$ H.F. Robey, J.O. Kane, B.A. Remington, R.P. Drake, O.A. Hurricane, et al., Phys. Plasmas 8, 2446 (2001).

${ }^{24}$ R.P. Drake, H.F. Robey, O.A. Hurricane, Y. Zhang, B.A. Remington, et al., Astrophys. J. 564, 896 (2002).

${ }^{25}$ H.F. Robey, Y. Zhou, A.C. Buckingham, P. Keiter, B.A. Remington, et al., Phys. Plasmas 10, 614 (2003).

${ }^{26}$ E. M. Campbell, Laser Part. Beams, 9(2), 209 (1991).

${ }^{27}$ L. H. Howell and J.A. Greenough, J. Comp. Phys. 184, 53 (2003).

${ }^{28}$ E. M. Corey and D. A. Young, UCRL-JC-127698. Lawrence Livermore National Laboratory, Livermore, CA (1997).

${ }^{29}$ G. B. Zimmerman and W. L. Kruer, Comments on Plasma Physics and Controlled Fusion 2, 51 (1975).

${ }^{30}$ S. W. Haan, Phys. Fluids B 3(8), 2349 (1991).

${ }^{31}$ S. W. Haan, Phys. Rev. A 39(11), 5812 (1989).

${ }^{32}$ D. Ofer, U. Alon, D. Shvarts, R. L. McCroy, and C. P. Verdon, Phys. Plasmas 3(8), 
3073 (1996).

${ }^{33}$ D. L. Youngs, Phys. Fluids A 3(5), 1312 (1991).

${ }^{34}$ G. Dimonte, Phys. Plasmas 7(6), 2255 (2000).

${ }^{35}$ U. Alon, D. Shvarts, and D. Mukamel, Phys. Rev. E, 48(2), 1008 (1993).

${ }^{36} \mathrm{~A}$. R. Miles, "Bubble merger model for the nonlinear Rayleigh-Taylor instability driven by a strong blast wave", submitted to Phys. Plasmas; Chapter 4, this dissertation.

${ }^{37}$ R. H. Kraichnan, Phys. Fluids 10, 1417 (1967).

${ }^{38}$ M. A. Rutgers, Phys. Rev. Lett. 81(11), 2244 (1998).

${ }^{39}$ D. Oron, L. Arazi, D. Kartoon, A. Rikanati, U. Alon, and D. Shvarts, Phys. Plasmas 8(6), 2883 (2001).

${ }^{40}$ D. L. Youngs, Las. Part. Beams 12(4), 725 (1996).

${ }^{41}$ A. W. Cook, W. H. Cabot, P. L. Miller, P. E. Dimotakis, and T. W. Mattner, "Effects of mixing on Rayleigh-Taylor instability"

${ }^{42}$ P. E. Dimotakis, J. Fluid Mech. 409, 69 (2000).

${ }^{43}$ A. R. Miles, M.J. Edwards, B. Blue, J. F. Hansen, H.F. Robey, et al., "The effect of a short-wavelength mode on the evolution of a long wavelength perturbation driven by a strong blast wave", submitted to Phys. Plasmas; Chapter 3, this dissertation. ${ }^{44}$ K. Kifonidis, T. Plewa, H.-Th. Janka, and E. Muller, Astron. Astrophys. 408, 621 (2003).

${ }^{45}$ J. L. Milovich, P. Amendt, M. Marinak, and H. Robey, Phys. Plasmas 11(4), (2004).

${ }^{46}$ D. Ofer, D. Shvarts, Z. Zinamon, and S. A. Orszag, Phys. Fluids B 4(11), 3549 (1992). 
${ }^{47}$ D. M. Snider and M. J. Andrews, Phys. Fluids 6(10), 3324 (1994).

${ }^{48}$ J. Glimm, X. L. Li, R. Menikoff, D. H. Sharp, and Q. Zhang, Phys. Fluids A 2(11), 2046 (1990).

${ }^{49}$ D. L. Youngs, Laser and Part. Beams 12(4), 725 (1994).

\section{$\underline{\text { Chapter } 6}$}

${ }^{1}$ J. W. S. Rayleigh, Scientific Papers (University press, Cambridge, 1899).

${ }^{2}$ G. I. Taylor, Proc. R. Soc. London, Ser. A 201, 192 (1950).

${ }^{3}$ R. D. Richtmyer, Commun. Pure Appl. Math. 13, 297 (1960).

${ }^{4}$ E. E. Meshkov, Izv. AN SSSR Mekhanika Zhidkosti I Gaza 4(5), 151 (1969).

${ }^{5}$ A. R. Miles, D.G. Braun, M.J. Edwards, H.F. Robey, R.P. Drake, et al., "Numerical Simulation of Supernova-Relevant Laser-driven Hydro Experiments on OMEGA”, to appear in Phys. Plasmas; Chapter 2, this dissertation.

${ }^{6}$ A. R. Miles, M. J. Edwards, and J. A. Greenough, "Effect of Initial Conditions on 2D Rayleigh-Taylor Instability and Transition to Turbulence in Planar Blast-wave-driven Systems", submitted to Phys. Plasmas; Chapter 5, this dissertation.

${ }^{7}$ L. H. Howell and J.A. Greenough, J. Comp. Phys. 184, 53 (2003).

${ }^{8}$ K. Kifonidis, T. Plewa, H.-Th. Janka, and E. Muller, Astron. Astrophys. 408, 621 (2003).

${ }^{9}$ H. Tennekes and J. L. Lumley, A First course in Turbulence (The MIT Press, Cambridge, 1972).

${ }^{10}$ P. E. Dimotakis, J. Fluid Mech. 409, 69 (2000).

${ }^{11}$ G. G. Stokes, Cambr. Phil. Trans. IX, 8 (1851). 
${ }^{12}$ D. Galmiche and S. Gauthier, Jpn. J. Appl. Phys. 35, 4516 (1996).

${ }^{13}$ H. F. Robey, Ye Zhou, A. C. Buckingham, P. Keiter, B. A. Remington, and R. P.

Drake, Phys. Plasmas 10, 614 (2003).

${ }^{14}$ Ye Zhou, H. F. Robey, A. C. Buckingham, Phys. Rev. E 67, 056305 (2003).

${ }^{15}$ R. H. Kraichnan, Phys. Fluids 10, 1417 (1967).

${ }^{16}$ M. A. Rutgers, Phys. Rev. Lett. 81(11), 2244 (1998).

${ }^{17}$ S. E. Widnall, D. B. Bliss, and C. Tsai, J. Fluid Mech. 66(1), 35 (1974).

${ }^{18}$ K. I. Read, Physica D, 12, 45 (1984).

${ }^{19}$ D. L. Youngs, Physica D, 12, 32 (1984).

${ }^{20}$ U. Alon, J. Hecht, D. Mukamel, and D. Shvarts, Phys. Rev. Lett., 72(18), 2867 (1994).

${ }^{21}$ J. Glimm, D. H. Sharp, Phys. Rev. Lett. 64(18), 2137 (1990).

${ }^{22}$ A. R. Miles, "Bubble merger model for the nonlinear Rayleigh-Taylor instability driven by a strong blast wave", submitted to Phys. Plasmas; Chapter 4, this dissertation.

${ }^{23}$ D. L. Youngs, Laser and Part. Beams 12(4), 725 (1994).

${ }^{24}$ A. W. Cook, W. H. Cabot, P. L. Miller, P. E. Dimotakis, and T. W. Mattner, "Effects of mixing on Rayleigh-Taylor instability", in preparation.

${ }^{25}$ D. Layzer, Ap. J. 122, 1 (1955).

${ }^{26}$ M. A. Jones and J. W. Jacobs, Phys. Fluids 9, 3078 (1997); B. D. Collins and J. W. Jacobs, J. Fluid Mech. 464, 113 (2002).

${ }^{27}$ G. Dimonte and M. Schneider, Phys. Fluids 12(2), 304 (2000).

${ }^{28}$ E. M. Campbell, Laser Part. Beams, 9(2), 209 (1991). 
${ }^{29}$ G. B. Zimmerman and W. L. Kruer, Comments on Plasma Physics and Controlled Fusion 2, 51 (1975).

${ }^{30}$ R. M. Davies and G. I. Taylor, Proc. R. Soc. London Ser. A 200, 375 (1950).

${ }^{31}$ D. Oron, L. Arazi, D. Kartoon, A. Rikanati, U. Alon, and D. Shvarts, Phys. Plasmas 8(6), 2883 (2001).

${ }^{32}$ A. R. Miles, M.J. Edwards, B. Blue, J. F. Hansen, H.F. Robey, et al., "The effect of a short-wavelength mode on the evolution of a long wavelength perturbation driven by a strong blast wave", submitted to Phys. Plasmas; Chapter 3, this dissertation.

${ }^{33}$ D. L. Youngs, Phys. Fluids A 3(5), 1312 (1991).

${ }^{34}$ D. L. Youngs, Las. Part. Beams 12(4), 725 (1996).

${ }^{35}$ J. A. Greenough, (private communication).

${ }^{36}$ A. N. Kolmogorov, Dokl. Akad. Nauk SSSR 31, 538 (1941).

${ }^{37}$ A. M. Oboukhov, Izv. Akad. Nauk SSSR Ser. Georgr. Geofiz. 13, 58 (1949).

${ }^{38}$ S. Corrsin, J. Appl. Phys. 22, 4 (1951).

\section{Appendix A}

${ }^{1}$ R. D. Richtmyer, Commun. Pure Appl. Math. 13, 297 (1960).

${ }^{2}$ E. E. Meshkov, Izv. AN SSSR Mekhanika Zhidkosti I Gaza 4(5), 151 (1969).

${ }^{3}$ K. A. Meyer and P. J. Blewett, Phys. Fluids 15(5), 753 (1972).

${ }^{4}$ J. W. Jacobs and J. M. Sheeley, Phys. Fluids 8(2), 405 (1996).

${ }^{5}$ Y. Yang, Q. Zhang, and D. Sharp, Phys. Fluids 6(5), 1856 (1993).

${ }^{6}$ A. L. Velikovich, Phys. Fluids 8(6), 1666 (1996).

${ }^{7}$ U. Alon et al., Phys. Rev. Lett. 72(18), 2867 (1994). 
${ }^{8}$ J. Hecht, U. Alon, and D. Shvarts, Phys. Fluids 6(12), 4019 (1994).

${ }^{9}$ U. Alon et al, Phys. Rev. Lett. 74(4), 534 (1995).

${ }^{10}$ A. Rikanati, U. Alon, and D. Shvarts, Phys. Rev. E 58(6), 7410 (1998).

${ }^{11}$ A. Rikanati et al, Ap. J. Supplemental Series 127, 451 (2000).

${ }^{12}$ D. Oron et al, Phys. Plasmas 8(6), 2883 (2001).

${ }^{13}$ J. C. V. Hanson et al., Laser Part. Beams 8, 51 (1990).

${ }^{14}$ G. Dimonte, Phys. Plasmas 7(6), 2255 (2000).

${ }^{15} \mathrm{H}$. Robey, "The effect of shock proximity on the non-linear evolution of RichtmyerMeshkov instability at high Mach number," submitted to Phys. Fluids.

${ }^{16}$ A. L. Velikovich and G. Dimonte, Phys. Rev. Lett. 76(17), 3112 (1996).

${ }^{17} \mathrm{Q}$. Zhang and S. Sohn, Phys. Fluids 9(4), 1106 (1997).

${ }^{18}$ O. Sadot et al., Phys. Rev. Lett. 80(8), 1654 (1998).

${ }^{19}$ G. Dimonte and B. Remington, Phys. Rev. Lett. 70(12), 1806 (1993).

${ }^{20}$ G. Dimonte et al, Phys. Plasmas 3(2), 614 (1996).

${ }^{21}$ R. L. Holmes et al., J. Fluid Mech. 389, 55 (1999).

${ }^{22}$ D. R. Farley et al., Phys. Plasmas 6(11), 4304 (1999).

${ }^{23}$ J. Lindl, Inertial Confinement Fusion: the quest for ignition and high-energy gain, (Springer-Verlag, New York, 1998).

${ }^{24}$ G. Glendinning et al., Phys. Plasmas 10(5). 1931 (2003).

${ }^{25}$ O. A. Hurricane et al, Phys. Fluids 12, 2148 (2000).

${ }^{26}$ G. BenDor et al., (Academic Press, London, 2001), Vol. 2, p. 489.

${ }^{27}$ R. T. Barton, Numerical Astrophysics (Jones and Bartlett, Boston, 1985).

${ }^{28} \mathrm{~A}$. Miles et al., "Numerical simulation of supernova-relevant laser-driven hydro 
experiments on OMEGA", to appear in Phys. Plasmas; Chapter 2, this dissertation. ${ }^{29}$ G. B. Zimmerman and W. L. Kruer, Comm. Plasma Phys. 11, 51 (1975).

${ }^{30}$ A. N. Aleshin, S. G. Zaitsev, and E. V. Lazareva, Rus. J. Comp. Mech. 1(2), 33 (1993).

${ }^{31}$ M. M. Marinak et al., Phys. Rev. Lett. 75, 3677 (1995).

${ }^{32}$ L. H. Howell and J.A. Greenough, J. Comp. Phys. 184, 53 (2003).

${ }^{33}$ J.W. S. Rayleigh, Scientific Papers (University press, Cambridge, 1899).

${ }^{34}$ G. I. Taylor, Proc. R. Soc. London, Ser A 201, 192 (1950). 